\title{
A MEASUREMENT OF THE MUON NEUTRINO CHARGED CURRENT QUASIELASTIC-LIKE CROSS SECTION ON A HYDROCARBON TARGET AND FINAL STATE INTERACTION EFFECTS
}

\author{
A Dissertation \\ By \\ TAMMY WALTON
}

Submitted to the Graduate College of Hampton University in partial fulfillment of the requirements for the degree of

DOCTOR OF PHILOSOPHY

May 2014 
This dissertation submitted by Tammy Walton in partial fulfillment of the requirements for the degree of Doctor of Philosophy at Hampton University, Hampton, Virginia is hereby approved by the committee under whom the work has been completed.

M. Eric Christy, Ph.D.

Committee Chair

Jóse L. Goity, Ph.D.

Cynthia Keppel, Ph.D.

Jeffery K. Nelson, Ph.D.

Patrena N. Benton, Ph.D.

Dean, The Graduate College

Date 
Copyright by

TAMMY WALTON

2014 


\title{
ABSTRACT
}

\author{
A MEASUREMENT OF THE MUON NEUTRINO CHARGED CURRENT \\ QUASIELASTIC-LIKE CROSS SECTION ON A HYDROCARBON TARGET \\ AND FINAL STATE INTERACTION EFFECTS. (May 2014) \\ Tammy Walton, B.S., University of Tennessee \\ Ph.D., Hampton University \\ Chair of Advisory Committee: Dr. M. Eric Christy
}

Presented is the analysis of the $\nu_{\mu}$ charged-current quasielastic-like interaction with a polystyrene ( $\mathrm{CH}$ or hydrocarbon) target in the MINER $\nu \mathrm{A}$ experiment, which was exposed to a neutrino beam that peaked at $\sim 3.5 \mathrm{GeV}$. In neutrino scattering, the quasielastic interaction is defined as the process in which a neutrino scatters from a nucleon bound in the nucleus and via the exchanged of a $W^{ \pm}$boson, a charged lepton and nucleon are emitted. The quasielastic-like is defined as the neutrino-nucleus scattering that results in an event with the quasielastic topology. MINER $\nu \mathrm{A}$ is a dedicated neutrino-nucleus cross section experiment, which has published differential cross sections, $\mathrm{d} \sigma / \mathrm{dQ}_{\mathrm{QE}}^{2}$, measurements for both the $\nu_{\mu}$ and $\bar{\nu}_{\mu}$ quasielastic (QE) scattering [1], [2]. The datasets from both the $\nu_{\mu}$ and $\bar{\nu}_{\mu}$ QE reactions observed additional strength in the measured cross sections, which has been attributed to the 2-particle 2-hole meson exchange currents, which is beyond the description of the independent nucleon impulse approximation. The event kinematics for these measurements were produced assuming quasielastic scattering from a single nucleon and using only the muon reconstructed observables. To understand the kinematical correlations between the multinucleons, it is critical that both the lepton and nucleon(s) kinematics are measured. In addition, using the quasielastic hypothesis to reconstruct the neutrino energy purely from the lepton is inaccurate for scattering from multinu- 
cleons. The neutrino energy is directly related to the neutrino oscillation parameters. Therefore, it is essential that the kinematics from both the lepton and hadron systems are accurately modeled.

For scattering from a nucleus heavier than Hydrogen, the recoil hadrons can interact with the spectator nucleons before escaping the nucleus. As a result, the effects from final state interactions become significant, where final state interactions can obscure the expected kinematical corrrelations between the recoil nucleons. To improve the knowledge concerning final state interactions for neutrino scattering from a nucleon bound in a nucleus, this analysis measures the $\nu_{\mu}$ quasielastic-like differential cross section where the emitted muon and at least one proton are detected. 
The representation of this body of work extends far beyond the sleepless nights, unexpected sacrifices, and new horizons. Foremost, it symbolizes that to live does not mean to be content with the escape from the trenches of death and destruction, but to reach for everything and beyond the stars that this world has to offer. With the greatest honor, I dedicate this thesis to those who know me best, my siblings. 


\section{ACKNOWLEDGEMENTS}

Like all quests that we choose to pursue in life, we never have to walk alone. It is a pleasure to acknowledge those who have made this journey worth traveling.

I am grateful to Kevin McFarland and Debbie Harris for providing me with travel assistance and addressing my concerns about a few unexpected hurdles. Kevin, who offered me my first job, invitation for public lecture, and recognized the unlimiting potential within me, I will forever be in your debt. Bob Bradford and Jesse Chvojka thank you for extending a warm, opening, and friendly hand to me. It's difficult for me to feel like I belong, and it was awesome that you did your best to make it happen. I would like to recognize the amazing work done by Arturo Fiorentini, David Schmitz, and Laura Fields, your work has been my guiding light. A special shout out to Laura, thank you for always extending a helping hand. I am very much appreciative to each and every MINER $\nu$ An, both the past and the present, from the engineers to the shifters, all persons who contributed to this analysis in any form or shape. And thank you to Kenyi Hurtado, Jyotsna Osta, and Minerba Betancourt for the specific work you have done on this analysis. And especially to Minerba, thank you for always going beyond the call of duty. Thank you very much to Jan Sobczyk for your significant contributions, as well. Also, I am very glad to have worked along the side of Brandon Eberly, Brian Tice, and Benjamin Ziemer.

Mrs. Rashinda Davis, I am more than grateful for all you have done. You made life so much easier for all of us at Hampton University and I owe you more than words can express. A big thank you to my committee members, Jose Goity, Cynthia Keppel, and Jeff Nelson. With no introduction needed, Dr. Eric Christy thank you for never closing your door to any of my questions. The weight of my graduate career has been lightly carried due to your assistance and support. Thank you very much for being my advisor. 


\section{TABLE OF CONTENTS}

Section

Page

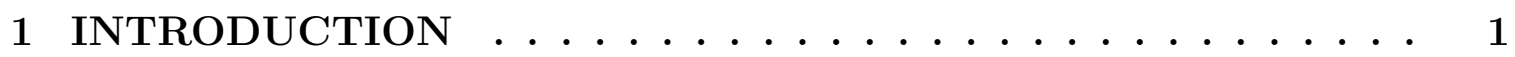

1.1 HISTORICAL OVERVIEW OF THE NEUTRINO . . . . . . . . . . 4

1.2 THE QUARK FORMALISM IN THE STANDARD MODEL . . . . . 6

1.2.1 ADDITIONAL PROPERTIES OF QUARKS . . . . . . . 7

1.3 THE WEAK NUCLEAR INTERACTION . . . . . . . . . . . . . . . 10

1.4 NEUTRINO OSCILLATIONS . . . . . . . . . . . . . . . 17

2 NEUTRINO CHARGED CURRENT QUASI-ELASTIC SCATTER-

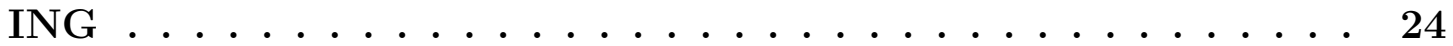

2.1 INTRODUCTION TO ELASTIC SCATTERING . . . . . . . . . . . 24

2.2 NEUTRINO QUASI-ELASTIC SCATTERING . . . . . . . . . . 29

2.2.1 THE NEUTRINO QUASI-ELASTIC DIFFERENTIAL CROSSSECTION ................................. 30

2.2.2 THE VECTOR FORM FACTORS . . . . . . . . . . . . . . . 32

2.2.3 THE NUCLEAR $\beta$-DECAY HADRONIC CURRENT . . . . . 33

2.2.4 THE PSEUDOSCALAR FORM FACTOR . . . . . . . . . 35

2.2.5 THE AXIAL FORM FACTOR . . . . . . . . . . . . 37

2.3 SCATTERING FROM HEAVIER NUCLEI . . . . . . . . . . . . . . 39

2.3.1 NUCLEON-NUCLEON SHORT RANGE CORRELATIONS . 42

2.3.2 Meson Exchange Currents . . . . . . . . . . . . . . 43

2.3.3 THE EFFECTS ON RECONSTRUCTION . . . . . . . . . 45

2.4 THE EFFECTS OF THE FINAL STATE INTERACTIONS . . . . . 46

3 THE MINER $\nu$ A EXPERIMENT . . . . . . . . . . . . 48 
3.1 THE NuMI BEAMLINE . . . . . . . . . . . . . . . . . . . . . 48

3.1.1 THE PROTON BEAM . . . . . . . . . . . . . . . . . 49

3.1 .2 THE NuMI APPARATUS . . . . . . . . . . . . . 50

3.2 THE MINER $\nu$ A DETECTOR . . . . . . . . . . . . . 54

3.2.1 INNER DETECTOR . . . . . . . . . . . . . . 55

3.2.2 OUTER DETECTOR . . . . . . . . . . . . . 61

3.2.3 UPSTREAM REGION . . . . . . . . . . . . . . . . . . 62

3.2.4 THE SCINTILLATOR STRIPS . . . . . . . . . . . . 63

3.2.5 THE PHOTOMULTIPLIER TUBE AND OPTICAL BOX . . 64

3.2.6 READOUT ELECTRONICS AND THE DAT ACQUISITION

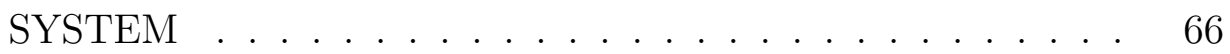

3.3 THE MINOS NEAR DETECTOR . . . . . . . . . . . 67

3.4 THE DATA RUN PERIOD . . . . . . . . . . . . . . . . . . 68

4 CAlibrations, SIMULTIONS, AND NEUTRINO EVENT RE-

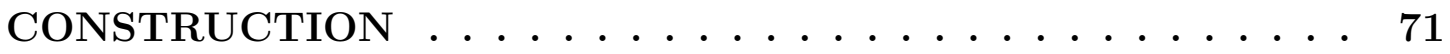

4.1 CALIBRATION . . . . . . . . . . . . . . . . 73

4.1.1 Ex situ Calibration . . . . . . . . . . . . . . . 73

4.1.2 In situ Calibration . . . . . . . . . . . . . . . . . 76

4.2 SIMULATION . . . . . . . . . . . . . . . . . 85

4.2 .1 NuMI FLUX . . . . . . . . . . . . . . . . . 85

4.2.2 NEUTRINO EVENT GENERATOR . . . . . . . . . . . . . 90

4.2.3 SIMULATING EVENTS IN THE DETECTOR . . . . . . . . 95

4.3 RECONSTRUCTION ........................ 96

4.3.1 THE CLUSTERING OF HITS . . . . . . . . . . . . . . . 98

4.3.2 TRACK RECONSTRUCTION . . . . . . . . . . . . . 101 
4.3.3 VERTEX RECONSTRUCTION . . . . . . . . . . . . 109

4.3.4 MUON RECONSTRUCTION . . . . . . . . . . . . . . 109

4.3.5 HADRON RECONSTRUCTION . . . . . . . . . . 116

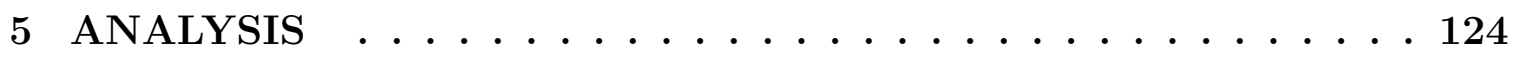

5.1 SELECTING $\nu_{\mu}$ CHARGED CURRENT EVENTS . . . . . . . . . 125

5.2 ISOLATING THE $\nu_{\mu}$ QUASIELASTIC-LIKE EVENTS $\quad \ldots . .128$

5.2.1 THE APPLIED ANALYSIS CUTS . . . . . . . . . . . . 129

5.2.2 THE $\nu_{\mu}$ QUASIELASTIC-LIKE CANDIDATES . . . . . . . . 137

5.3 MEASURING THE CROSS-SECTION . . . . . . . . . . . . . . . 145

5.3.1 BACKGROUND SUBTRACTION . . . . . . . . . . 145

5.3 .2 BIN MIGRATION AND UNFOLDING . . . . . . . . . . 158

5.3.3 EFFICIENCY AND ACCEPTANCE CORRECTIONS . . . . 161

5.3.4 DIFFERENTIAL CROSS-SECTION RESULTS . . . . . . . . 165

5.4 SYSTEMATIC UNCERTAINTIES . . . . . . . . . . . . 175

5.4.1 NEUTRINO FLUX . . . . . . . . . . . . . . . 176

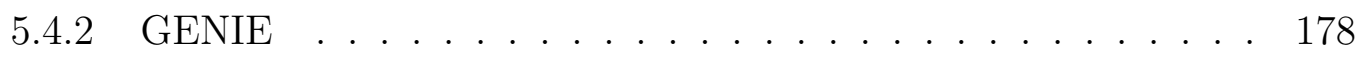

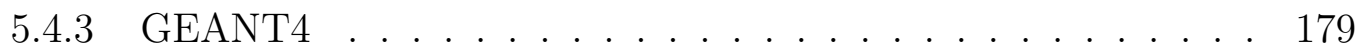

5.4.4 PROTON RECONSTRUCTION . . . . . . . . . . . 184

5.4.5 MICHEL ELECTRON VETO . . . . . . . . . . . . . . 187

5.4.6 OTHER SYSTEMATIC UNCERTAINTIES . . . . . . . . . 188

5.5 STUDYING NUCLEAR EFFECTS USING BOTH THE MUON AND PROTON KINEMATICS . . . . . . . . . . . . . . . . 190

5.5.1 GENIE INTERPRETATION OF NUCLEAR EFFECTS . . . 191

5.5.2 THE MUON-PROTON KINEMATICS ANALYSIS . . . . . . 194 
6 RESULTS AND CONCLUSIONS . . . . . . . . . . 197

6.1 COMPARING MINER $\nu \mathrm{A} \mathrm{d} \sigma / \mathrm{dQ}_{\mathrm{QE}}^{2}$ MEASUREMENTS . . . . . . 197

6.2 MODEL COMPARISONS OF THE MEASURED d $\sigma / \mathrm{dQ}_{\mathrm{QE}-\text { like,proton }}^{2} 201$ 6.2.1 INTERPRETING THE d $\sigma / \mathrm{dQ}_{\mathrm{QE} \text {-like,proton }}^{2}$ MEASUREMENT 205

6.3 THEORETICAL PREDICTIONS FOR EXAMINING THE EFFECTS OF FSI . . . . . . . . . . . . . . . . . . 214

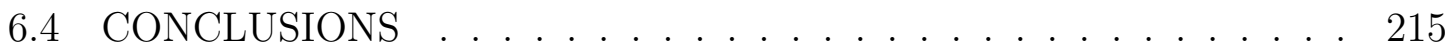

\section{APPENDICES}

A $\nu_{\mu}$ QE-LIKE KINEMATIC DISTRIBUTIONS $\ldots . \ldots . . \ldots$

B MUON-PROTON $\nu_{\mu}$ QE-LIKE KINEMATIC DISTRIBUTIONS 240

C $\nu_{\mu}$ QE-LIKE EFFICIENCY DISTRIBUTIONS . . . . . . . 245

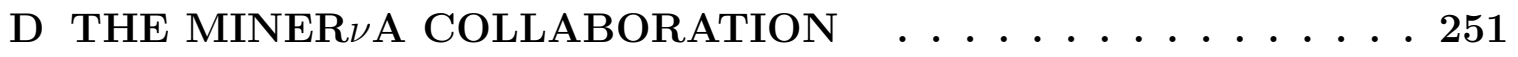

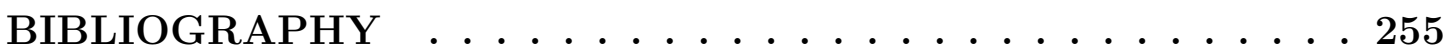




\section{LIST OF TABLES}

Table

Page

1.1 The Quark Family of spin $=\frac{1}{2} \ldots \ldots \ldots \ldots$

1.2 The Lepton Family of spin $=\frac{1}{2} \ldots \ldots \ldots$. . . . . . . . . . 7

2.1 The Published $\mathrm{M}_{A}$ values by $\nu$-deuterium Experiments. Reprinted from Reference [30], Copyright (2007). . . . . . . . . . . . . . 38

3.1 The Element Composition of the Scintillator Plane by Mass Percentage. 57

3.2 The Nuclear Targets Material and Geometrical Description. . . . . . . . 61

4.1 A summary of the kinematic regimes for re-weighting the hadron pro-

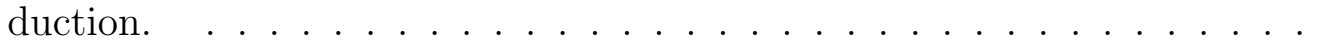

4.2 The Relativistic Fermi Gas Model parameters for the Quasielastic Scattering. ........................... 91

4.3 The Nuclear Interaction Lengths of Materials in MINER $\nu$ A . . . . . . . 116

5.1 The Event Rate for Selecting $\nu_{\mu}$ Charged Current Events. . . . . . . . . 127

5.2 The Event Rate for Isolating the $\nu_{\mu}$ QE-like Candidates. $\quad \ldots . . . . \quad 138$

5.3 The Muon Topology Rate for the Simulated Events. . . . . . . . . . . . 139

5.4 Flux-integrated $\mathrm{d} \sigma / \mathrm{dQ}_{\mathrm{QE}-\text { like,proton }}^{2}$ Summary. Both the statistical (first) and absolute systematic (second) errors are presented for each measurement. 170

5.5 Fractional systematic uncertainties on the $\mathrm{d} \sigma / \mathrm{dQ}_{\mathrm{QE}-\text { like,proton measure- }}^{2}$ ment for each $\mathrm{Q}_{\mathrm{QE} \text {,proton }}^{2}$ bin. Both the absolute (top) and area (bottom) errors are presented for each $\mathrm{Q}_{\mathrm{QE} \text {,proton }}^{2}$ bin and the contributors are (I) flux, (II) neutrino interaction models, (III) final state interaction models, (IV) detector response, (V) energy response, (VI) proton reconstruction, (VII) Michel electron veto, and (VIII) other . . . . . . . . . . . . 170

5.6 Fractional systematic uncertainties on the $\mathrm{d} \sigma / \mathrm{dQ}_{\mathrm{QE}-\text { like,proton measure- }}^{2}$ ment with $\mathrm{p}_{\text {proton }}>450 \mathrm{MeV} / \mathrm{c}$ for each $\mathrm{Q}_{\mathrm{QE} \text {,proton }}^{2}$ bin. Both the absolute (top) and shape (bottom) errors are presented for each $\mathrm{Q}_{\mathrm{QE} \text {,proton }}^{2}$ bin and 
the contributors are (I) flux, (II) neutrino interaction models, (III) final state interaction models, (IV) detector response (V) energy response, (VI) proton reconstruction, (VII) Michel electron veto, and (VIII) other

5.7 Flux-integrated $\mathrm{d} \sigma / \mathrm{dQ}_{\mathrm{QE}-\text { like,proton }}^{2}$ Summary for $\mathrm{p}_{\text {proton }}>450 \mathrm{MeV} / \mathrm{c}$. Both the statistical (first) and full systematic (second) errors are presented for each measurement. . . . . . . . . . . . . . . . . . 174

5.8 The systematic uncertainties on various sources of the NuMI beamline. Reprinted from Reference [95], Copyright (2008). . . . . . . . . . . . . . 177

5.9 The systematic uncertainties on various parameters for GENIE models. Reprinted from Reference [75], Copyright (2010). . . . . . . . . . . . . . 180

5.10 The E $\mathrm{E}_{\text {unattach }}^{\text {Eis }}$ Energy Response Uncertainties. . . . . . . . . . . . . . . . 182

5.11 The Mass Thickness Uncertainties for Material in the Tracker Region. Reprinted from Reference [102]. . . . . . . . . . . . . . . . 186

6.1 Flux-integrated $\mathrm{d} \sigma / \mathrm{dQ}_{\mathrm{QE}}^{2}$ Summary for the inclusive and exclusive $\mathrm{QE}$ analyses. Both the statistical (first column) and systematic (second column) errors are presented for each measurement. The one-track cross section is reprinted from Reference $[1] \ldots . . . . . . . .$.

6.2 The fractional systematic uncertainties on the one-track (top) and $n$ track (bottom) QE cross section measurements for each $\mathrm{Q}_{\mathrm{QE}, \mathrm{muon}}^{2}$ bin. The contributors are the (I) flux, (II) neutrino interaction models, (III) final state interaction models, (IV) muon reconstruction (V) recoil reconstruction, (VI) other. The one-track uncertainties are reprinted from Reference [1]. . . . . . . . . . . . . . . . . . . . . . 202

6.3 Calculated $\chi^{2}$ between the measured $\mathrm{d} \sigma / \mathrm{dQ}_{\mathrm{QE}-\text { like,proton }}^{2}$ and various models with $\mathrm{M}_{A}=0.99 \mathrm{GeV} / \mathrm{c}^{2}$ and the $\mathrm{p}_{\text {proton }}>450 \mathrm{MeV} / \mathrm{c}$. The degrees of freedom, d.o.f is 7 (6) for the rate (shape) $\chi^{2}$ calculation. . . . . . . . 211

6.4 The model to the measured data $\mathrm{d} \sigma / \mathrm{dQ}_{\mathrm{QE}-\text { like,proton }}^{2}$ area normalization constant. . . . . . . . . . . . . . . . . . 213 


\section{LIST OF FIGURES}

Figure

Page

1.1 The Feynman diagram of Enrico Fermi's proposal of the four-point $\beta$-decay. 4

1.2 The Feynman diagram of the inverse $\beta$-decay. The arrows point backwards for the anti-particles, where anti-particles can be interpreted as moving backwards in time. . . . . . . . . . . . . . . . . . .

1.3 A Feynman diagram of H. Yukawa's proposal of the intermediate particle, W gauge boson, in the $\beta$-decay. . . . . . . . . . . . . . . . 6

1.4 The Feynman diagram of the quark model of the $\beta$-decay. . . . . . . . . 10

1.5 The Feynman diagram of the four-point $\beta$-decay where the arrows represent the structure of the weak currents at the hadronic and leptonic vertices.

1.6 The $\mathrm{W}^{ \pm}$quark vertices for lepton-quark symmetry, excluding the quark mixing. . . . . . . . . . . . . . . . . . . 16

1.7 The quark mixing interpretation of the $\mathrm{u}+\mathrm{d}^{\prime} \rightarrow \mathrm{W}$ vertex. . . . . . . 17

1.8 The hierarchical structure of the mass splitting in neutrino oscillations. 21

2.1 The Leading-order (single photon exchange) Quantum Electrodynamics (QED) Feynman diagram. . . . . . . . . . . . . . 25

2.2 The pion vertex correction diagram. . . . . . . . . . . . . . . . 34

2.3 The one-pion exchanged diagram. . . . . . . . . . . . . 35

2.4 The axial mass extraction from neutrino and anti-neutrino quasielastic scattering experiments. Reprinted from Reference [29], Copyright (2001). 37

2.5 The total QE cross section for the NOMAD and MiniBooNE experiments. Reprinted from [36], Copyright (2010) with permissions from MiniBooNE Collaboration.

2.6 The most recent $\mathrm{M}_{A}$ measurements on Heavy Nuclei. Reprinted from Reference [35], Copyright (2010) . . . . . . . . . . . . . . . 
Figure

Page

2.7 The Feynman diagrams of nucleon-nucleon correlations in the nuclear ground state. The wiggle lines, dotted lines, solid lines correspond to the virtual boson, pion, and the nucleons, respectively. The boson is absorbed by a correlated nucleon pair, where a pion is exchanged between the nucleons. Reprinted from Reference [41] with permissions from J. Sobczyk. . . . . . . . . . . . . . . . . .

2.8 Example Feynman diagrams of the meson exchange currents. The top Figures are known as the contact and pion-in-flight diagrams and the bottom Figures are called $\triangle$-MEC diagram. Reprinted from Reference [41] with permissions from J. Sobczyk. . . . . . . . . . . . . . .

2.9 The theoretical computation of the neutrino energy spread for three $\mathrm{E}_{\nu}$ values on the Carbon nucleus, where $\overline{\mathrm{E}}_{\nu}$ is the smeared value of the generated neutrino energy, $\mathrm{E}_{\nu}$. Reprinted from Reference [47], Copyright (2012)

3.1 A schematic of the NuMI beamline (not drawn to scale). Reprinted from [52], Copyright (2005), with permissions from R. Zwaska. . . . .

3.2 The NuMI production target and target vacuum canister, where the Target Segments are most commonly referred to as fins. The target is mounted on a rail-driver system with a maximum longitudinal travel length of $2.5 \mathrm{~m}$. Reprinted from Reference [53], Copyright (2002). . .

3.3 A schematic diagram of NuMI two focusing magnetic horns. Reprinted from Reference [55], Copyright (2005). . . . . . . . . . . . . . .

3.4 A schematic picture of th NuMI Hadron and Muon Monitors before the installation of the fourth muon alcove. Reprinted from Reference [53], Copyright (2006) with permissions from Elsevier. . . . . . . . . . . 54

3.5 A schematic diagram of the MINER $\nu \mathrm{A}$ detector as shown from the top view. Reprinted from [51], Copyright (2013). . . . . . . . . . . . . .

3.6 A schematic diagram of a tracking module mounted in an outer detector frame. Reprinted from [51], Copyright (2013). . . . . . . . . . . . 56

3.7 A schematic diagram of the ID scintillator plane orientated in the Xview. The left diagram shows the plane from the front view. The right figure shows the cross section of the triangular scintillator strips. Each strip contains a WLS fiber. . . . . . . . . . . . . . . . 57

3.8 A schematic diagram of the MINER $\nu \mathrm{A}$ coordinate system. . . . . . . 58 
3.9 A schematic diagram of the nuclear targets region in the MINER $\nu \mathrm{A}$ experiment, where the liquid cryotarget vessel is excluded. The Targets 1,2 , and 5 are composed of the iron and lead nuclei, Target 3 is composed of carbon, iron, and lead, and Target 4 is composed only of lead. The water target was not installed at the beginning of the LE run period. Reprinted from [53], Copyright (2013) with permissions from B. Tice. .

3.10 A schematic diagram of the cross section of an OD bar. An OD bar is composed of two rectangular scintillator strips with wavelength shifting fiber running through each strip. . . . . . . . . . . . . .

3.11 A picture of the components that make up the MINER $\nu \mathrm{A}$ PMT optical box. The PMT box is displayed from the back-end to the front-end. The ODU is positioned between the box's back-end and the black disc which mounts the fiber cookie. The PMT is mounted on a base circuit board and the cylindrical rods are used to ensure alignment. . . . . . . . . . 65

3.12 The MINOS near detector. The left (right) shows a top (beam) view of the detector. Reprinted from [65], Copyright (2010) with permissions from the MINOS Collaboration.

3.13 The MINER $\nu$ A total collected data for the Low Energy Run Period. The Special Runs correspond to the periods when the NuMI target-horn system was in a non-standard configuration. . . . . . . . . . .

4.1 A schematic diagram of an optical readout channel. Reprinted from [51], Copyright (2013). . . . . . . . . . . . . . . . . 71

4.2 A picture of the Module Mapper. Reprinted from [51], Copyright (2013).

4.3 A schematic diagram of the PMT test stand. Six PMTs, which are represented by the red cubes, are mounted onto fiber cookies which are represented by the yellow rectangular boxes. A bundle of six clear optical fibers is connected to six PMTs, such that one channel of each PMT can be read out using the same clear optical cable. Light from a blue LED is absorbed by a green WLS fiber and illuminates one pixel in each of the six PMTs, simultaneously. Reference [51], Copyright (2013). . . .

4.4 Plotted is an example of a high gain pedestal distribution for a single channel, where the outlier may originated from a cosmic ray event. Reprinted from Reference [51], Copyright (2013) . . . . . . . . . . 77 
Figure

4.5 The plot shows the average PMT gain as a function of time. The dip at day 120 is a result of adjusting the high voltages. Reprinted from Reference [51], Copyright (2013). . . . . . . . . . . . . . . . 78

4.6 A schematic diagram of a rock muon traversing two adjacent strips. Rock muons that travel throughout the detector usually deposited energy in one or two strips per plane. Reprinted from Reference [66], with courtesy from C. Marshall. . . . . . . . . . . . . . . . . . . . . . . 79

4.7 The plots show the scintillator plane alignment measurements as described in the text. Reprinted from Reference [51], Copyright (2013). . .

4.8 Results for all ID channels of the truncated mean energy loss before and after applying one iteration of the strip-to-strip response calibration. The uncalibrated data (red histogram) has a width of $\sigma=19.1 \%$ and after the first iteration the distribution (blue histogram) decreases to a width of $\sigma=1.6 \%$. Not shown on the plot, but the procedure is preformed in two iterations. Reprinted from Reference [66], with permissions from C. Marshall. . . . . . . . . . . . . . . . . . . .

4.9 The energy loss distributions of clusters on rock muon tracks. On the left is the simulated and data distributions and the right shows a fit to the peak of the data distribution. The left plot shows that the comparison between the simulated and measured energy loss of rock muon events in the detector is remarkable good. Reprinted from Reference [51], Copyright (2013). . . . . . . . . . . . . . . . .

4.10 The measured timing slew as a function of photoelectrons for throughgoing rock muons, as describe in the text. Reprinted from Reference [51], Copyright (2013). . . . . . . . . . . . . . . . .

4.11 Plotted is various neutrino energy spectrums using the same MC geometry, but different input hadronic physics models. The green arrow illustrates the discrepancy between the peaks of the distributions. Plot courtesy from M. Kordosky of the MINER $\nu$ A Collaboration. . . . . . .

4.12 The left (right) plot shows a comparison of the FTFP_BERT predicted cross section to the NA49 data for the $p C \rightarrow \pi^{+} X\left(p C \rightarrow \pi^{-} X\right)$ collisions. Plots courtesy of L. Aliaga of the MINER $\nu$ A Collaboration. . . .

4.13 (Left) $\nu_{\mu}$ and $\bar{\nu}_{\mu}$ simulated fluxes with and without the hadron production weights applied. (Right) Ratio of the hadron re-weighting distributions, where the dip region corresponds to the falling edge of the focusing peak. Plots are a courtesy of L. Aliaga of the MINER $\nu$ A Collaboration. . . . 
Figure

4.14 (Left) $\pi^{+} \mathrm{Fe}$ and (right) pFe cross sections which are utilized in the $h A$ INC model. The final state process is sampled from these data templates. Reprinted from Reference [79]. . . . . . . . . . . . . . .

4.15 An illustration of the differences between the $h A$ and $h N$ intranuke cascade models, as mentioned in the text. Reprinted from Reference [80]. .

4.16 A flowchart of the reconstructed software objects in the MINER $\nu \mathrm{A}$ framework. . . . . . . . . . . . . . . . 97

4.17 An example of the time profile of hits observed within a NuMI gate . . 98

4.18 An illustration of the cluster formation in an ID plane. The arrows represent the trajectories of particles and the different colors correspond to the density of the energy deposition. . . . . . . . . . . . . . .

4.19 An illustration of the cluster formation in an OD bar. For many cases, the clusters are composed of an one-hit strip. This is mainly because most of the particles which enter and may exit the OD have large angles with respect to the beam axis. . . . . . . . . . . . . . . .

4.20 An illustration of the general procedure for creating a track-based event for a high multiplicity neutrino interaction in the MINER $\nu \mathrm{A}$ detector, see the text for the description of the labels. . . . . . . . . . . .

4.21 Difference between the fitted positions relative to the measured cluster positions for rock muon events. Reprinted from [51], Copyright(2013). .

4.22 The simulated neutrino energy spectrum for the $\nu_{\mu}$ quasielastic-like channel, where the stack histograms correspond to the reconstructed muon track topology. . . . . . . . . . . . . . . . . . . . .

4.23 An illustration of the simple case for the OD energy reconstruction, where the energy is reconstructed using an iterative $d E / d x$ technique of the projected track. As shown, the stopping position of the projected track is determined by the most downstream cluster and the first bar which the projected track passes through. . . . . . . . . . . . . 115

4.24 An illustration of a more complicated case for stitching together all of the components of a hadron that propagates in the detector. Un-tracked energy around the vertex is not associated with the hadron prong. . . . 
4.25 Plotted is the $d E / d x$ profiles of the reconstructed track compared with the best fitted profiles for the proton and pion hypotheses. The plot shows that the proton best describes the reconstructed track. . . . . . . 120

5.1 Absolutely normalized data and Monte Carlo comparison of the number of tracks after applying each cut that was discussed earlier. One of the tracks is identified with the muon candidate and other tracks are declared as the hadron candidates. The Monte Carlo histograms are characterized by the signal (QE-like) and the non-signal (Resonant, DIS, other) events.

5.2 Absolute normalized data and Monte Carlo comparison of the proton pID score, which is interpreted as a particle ranging out score. The histograms are categorized by the Geant4 primary particle truth information. The discrepancy between the data and Monte Carlo is primarily due to the pion production cross section in GENIE. . . . . . . . . .

5.3 Absolutely normalized data and Monte Carlo comparison of the number of tracks after applying the pID cut. The pID cut essential removes all of the events with more the three tracks, which are dominated by the resonant and DIS events. . . . . . . . . . . . . . . .

5.4 Illustration of the detector regions in which the unattached visible energy is located. This illustration does not display any unattached visible energy. However, the energy that falls outside of the sphere with radius $=10 \mathrm{~cm}$ and is unassociated with a primary particle, makes up the unattached visible energy. . . . . . . . . . . . . . . . .

5.5 Absolute data and Monte Carlo comparison of the unattached visible energy on a linear (top) and log vertical (bottom) axis. The histogram contents are characterized by the analysis signal definition. . . . . . . . 133

5.6 Distribution of simulated events in $\mathrm{E}_{\text {unattach }}^{\text {vis }}$ and $\mathrm{Q}_{\mathrm{QE} \text {,proton }}^{2}$ for the signal (top) and non-signal (bottom) events, along with the $\mathrm{Q}_{\mathrm{QE}, \text { proton }}^{2}$ dependent cut. . . . . . . . . . . . . . . . . .

5.7 Absolutely normalized distribution for the data and Monte Carlo comparison of the number of tracks after applying the unattached visible energy cut. This cut primarily removes events that originate from the DIS process. . . . . . . . . . . . . . . . . . .

5.8 Histogram of the absolutely normalized data and Monte Carlo events with and without a Michel electron. The $\pi^{-}$s are more likely to be captured by a nucleus and the $\pi^{0} \rightarrow \gamma \gamma$. Using the Geant4 truth in- 
formation, the histogram shows the fraction of $\pi^{+}$events with a Michel

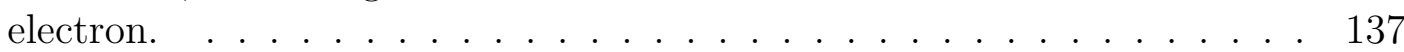

5.9 Absolutely normalized data and Monte Carlo comparison of the number of tracks after the vetoing event with a Michel electron. . . . . . . . . . 138

5.10 Distribution of the muon angle with respect to the beam axis absolutely normalized for the data and Monte Carlo comparison. See the text and Table 5.3 for more information describing the plot. . . . . . . . . . . .

5.11 Plotted is the muon momentum residual. The histogram is categorized by the reconstructed topology of the muon. The momentum reconstruction performs the best for the muons that are reconstructed and trackmatched by MINOS.

5.12 Histogram of the proton momentum residual for protons in the Tracker region of the MINER $\nu \mathrm{A}$ detector. . . . . . . . . . . . . . . . . . . . 141

5.13 The muon exits the MINER $\nu \mathrm{A}$ detector and is matched to a track in MINOS.

5.14 The muon is matched to energy in Outer Detector. . . . . . . . . . . . . 142

5.15 The $\nu_{\mu}$ QE-like candidates data and Monte Carlo area normalized (top) and absolute normalized (bottom) comparisons. The histogram makeup is characterized by the QE-like channel which includes the GENIE QE, Resonant, and DIS events with a final state that satisfies the defined signal. The non QE-like events are characterized by their GENIE definition. 143

5.16 The shape (top) and absolute (bottom) error summaries for the $\nu_{\mu}$ QElike candidates. For all plots presented in this dissertation, the shape errors corresponds to the errors on the area normalized distributions, where the shape error definition is given in Subsection 5.4 . . . . . . . . 144

5.17 The signal (left) and non-signal (right) simulated events for each sideband. The histograms illustrate the upper and lower cut boundaries for each sideband. . . . . . . . . . . . . . . . . . 147

5.18 Data and Monte Carlo absolute comparisons for each sideband. The (top) sideband1/sideband2 and (bottom) sideband3/sideband4 are characterized by the $\nu_{\mu}$ QE-like signal and GENIE definition of the backgrounds. 148

5.19 Extracted scale factors for each of the sideband, where the data to Monte Carlo event rate differ for each sideband. . . . . . . . . . . . . . . . . 150 
5.20 Linear fits for each $\mathrm{Q}_{\mathrm{QE} \text {,proton }}^{2}$ bin $i$, where the fit parameters give the scale factors for the two-component QE-like backgrounds. . . . . . . . . 152

5.21 The scale factors for the two-component QE-like backgrounds. . . . . . 153

5.22 The area (top) and absolutely (bottom) normalized data and Monte Carlo comparison for the QE-like candidates with the constrained backgrounds. For the absolutely normalized distribution, an enhancement in the data is observed. . . . . . . . . . . . . . . .

5.23 The area (top) and absolutely (bottom) normalized data and Monte Carlo comparison for the background subtracted distribution. The Monte Carlo only has statistical error bars. . . . . . . . . . . . . . . .

5.24 The shape (top) and absolute (bottom) error summary for the background subtracted distribution. The flux does not contribute to the systematic uncertainty on the background subtraction procedure. . . . . 157

5.25 Plotted is the smearing matrix, where the inverse of this matrix is defined as the unfolding matrix. The event in the underflow bin corresponds to neutrino interactions that have a proton with no kinetic energy in the final state, but a high energy neutron. The neutron immediately rescatters in the detector and knocks out a proton that is tracked. . . . .

5.26 The area (top) and absolutely (bottom) normalized data and Monte Carlo comparison for the unfolded distribution. . . . . . . . . . . . . 160

5.27 The shape (top) and absolute (bottom) error summaries for the unfolding procedure. . . . . . . . . . . . . . . . . .

5.28 (Top) Product of the Monte Carlo efficiency and acceptance for reconstructing the $\nu_{\mu}$ QE-like events and the (bottom) absolute systematic uncertainty on the efficiency function. Recall that the tracking threshold is responsible for the loss in efficiency for the low $\mathrm{Q}_{\mathrm{QE} \text {,proton }}^{2}$ bin, while the pID criteria and detector acceptance dominate in the high $\mathrm{Q}_{\mathrm{QE} \text {,proton }}^{2}$

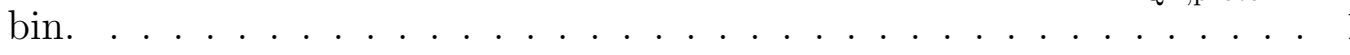

5.29 The efficiency-corrected area (top) and absolutely (bottom) normalized distributions. . . . . . . . . . . . . . . . . . 166

5.30 Plotted are both the shape (top) and absolute (bottom) error summaries for the efficiency-corrected distribution. . . . . . . . . . . . 167 
Figure

5.31 The area (top) and absolutely (bottom) normalized data and Monte Carlo comparison of the cross sections for the QE-like channel. . . . . . 168

5.32 The shape (top) and absolute (bottom) error summaries of the differential cross section. Notice for the absolute errors, the uncertainty on the flux becomes significant. . . . . . . . . . . . . . . .

5.33 The area (top) and absolutely (bottom) normalized data and Monte Carlo comparison of the cross sections for the QE-like channel. . . . . . 172

5.34 Plotted are the shape (top) and absolute (bottom) error summary for

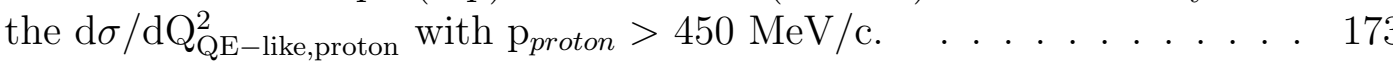

5.35 The predicted neutrino flux error summary. Recall that unlike other MINER $\nu \mathrm{A}$ analyses, this analysis does not cut on the reconstructed neutrino energy. . . . . . . . . . . . . . . . . . 178

5.36 Plotted are the most significant error sources for this analysis from GENIE cross section and final state interaction models. . . . . . . . . . . . 181

5.37 The cross section absolutely errors for each particle's source which contributes to the systematic uncertainty on the detector response for the unattached visible energy. . . . . . . . . . . . . . . . . . 183

5.38 The systematic uncertainties on the cross section that are due to the Geant4 modeling of the proton and pion inelastic cross sections. . . . . 184

5.39 Fractional uncertainty on the cross section due to various source that contribute to proton reconstruction systematic uncertainty. . . . . . . 186

5.40 The Michel electron veto systematic uncertainty. The systematic error on the Michel electron reconstruction is negligible. . . . . . . . . . . 188

5.41 The remaining contributions to the total systematic uncertainty. . . . . 189

5.42 Plotted are the correlations between the $\mathrm{Q}^{2}$ calculated using the event(red), muon(green), nucleon(blue), and proton(magenta and light gray) kinematics as a function of $\mathrm{Q}^{2}$ calculated from the event kinematic. Note that these are $\nu_{\mu}$ QE-like events without any applied cuts. See text for full description of the plot. Plot is a courtesy of M. Betancourt of the MINER $\nu \mathrm{A}$ Collaboration. . . . . . . . . . . . . . . . 192 
Figure

Page

5.43 The correlations between the $\mathrm{Q}^{2}$ which is calculated using the muon and proton kinematics that are derived from the QE scattering. Plot is courtesy of M. Betancourt of the MINER $\nu$ A Collaboration. . . . . . .

5.44 The absolutely comparisons of the reconstructed $\mathrm{Q}^{2}$ distributions using the muon (top) and proton (bottom) kinematics. The $\mathrm{Q}^{2}$ from the muon (which more accurately describe the true four-momentum transfer) shows that the backgrounds events populate the low $\mathrm{Q}^{2}$ regime. . . . . 196

6.1 The absolutely normalized one-track (orange) and n-track (blue) QE differential cross sections comparison. The one-track measurements are provided by Reference [1]. . . . . . . . . . . . . . . . . . . . 199

6.2 Ratio between the absolutely normalized one-track and n-track QE differential cross sections. The blue error band represents the total absolute systematic uncertainty on the $n$-track QE cross section. The inner errors bars on the data points show the total statistical errors for both analyses and the outer error bars show the total absolute systematic errors for the one-track QE cross section, respectively. . . . . . . . . . . . . 202

6.3 The one-track QE data and model comparison. The data prefers the RFG+TEM (dashed line) model which takes into account the enhancement in the cross section due to the knockout of multinucleons at the scattering vertex. Plot is courtesy of L. Fields of the MINER $\nu$ A Collaboration. . . . . . . . . . . . . . . . .

6.4 GENIE RFG (blue), NuWro RFG (red), and NuWro LFG (green) predictions of QE component of the QE-like cross section, with at least one proton having momentum greater than $450 \mathrm{MeV} / \mathrm{c}$ in the final state. . . 206

6.5 Ratio of the GENIE RFG (blue), NuWro RFG (red), and NuWro LFG (green) to GENIE RFG for the curves plotted in Figure 6.4.

6.6 GENIE (solid line) and NuWro (dashed line) comparison of the inelastic component of the QE-like cross section. . . . . . . . . . . . . . 207

6.7 Comparison of the absolutely normalized data and Monte Carlo with (solid line) and without (dashed line) FSI effects for the charged-current single pion production cross section as a function of the pion kinetic energy. Plot courtesy of B. Eberly of the MINER $\nu$ A Collaboration. . . 208

6.8 GENIE (solid line) and NuWro (dashed line) inelastic components of the QE-like cross section, where the GENIE resonant component has been scaled down by $30 \%$. 
Figure

Page

6.9 Plotted are the absolute (top) and shape (bottom) comparisons of the models to the data. The GENIE resonant component of the QE-like cross section has been scaled down by $30 \%$. . . . . . . . . . . . . . 210

6.10 Comparison of the area normalized data and Monte Carlo with (solid line) and without (dashed line) FSI effects for the charged-current single pion production cross section as a function of the pion kinetic energy. Plot courtesy of B. Eberly of the MINER $\nu$ A Collaboration. . . . . . . . 213

A.1 Plotted are the reconstructed vertex X, Y, and Z residuals. . . . . . . . 220

A.2 Plotted are the area (top) and absolutely (bottom) normalized reconstructed vertex X position. . . . . . . . . . . . . . . . 221

A.3 Plotted are the area (top) and absolutely (bottom) normalized reconstructed vertex Y position. . . . . . . . . . . . . . . . . . 222

A.4 Plotted are the area (top) and absolutely (bottom) normalized reconstructed vertex Z position. . . . . . . . . . . . . . . . . 223

A.5 Plotted are the reconstructed open angle and muon angle with respect to the beam axis residuals. . . . . . . . . . . . . . . . . . . . . . . . . 224

A.6 Plotted are the area (top) and absolutely (bottom) normalized reconstructed open angle between the muon and leading order proton distributions.

A.7 Plotted are the area (top) and absolutely (bottom) normalized reconstructed muon's polar angle with respect to the beam distributions. . . 226

A.8 Plotted are the area (top) and absolutely (bottom) normalized reconstructed muon's polar angle projected onto the x-axis with respect to the beam distributions.

A.9 Plotted are the area (top) and absolutely (bottom) normalized reconstructed muon's polar angle projected onto the y-axis with respect to the beam distributions. . . . . . . . . . . . . . . . . . . . . . . . . 228

A.10 Plotted are the area (top) and absolutely (bottom) normalized reconstructed muon's azimuthal angle with respect to the beam distributions.

A.11 Plotted are the reconstructed leading proton angles with respect to the beam axis residuals. . . . . . . . . . . . . . . . . . 230 
Figure

Page

A.12 Plotted are the area (top) and absolutely (bottom) normalized reconstructed leading proton's polar angle with respect to the beam axis distributions.

A.13 Plotted are the area (top) and absolutely (bottom) normalized reconstructed leading proton's polar angle projected onto the x-axis with respect to the beam axis distributions. . . . . . . . . . . . . . 232

A.14 Plotted are the area (top) and absolutely (bottom) normalized reconstructed leading proton's polar angle projected onto the y-axis with respect to the beam axis distributions. . . . . . . . . . . . . . . . . 233

A.15 Plotted are the area (top) and absolutely (bottom) normalized reconstructed leading proton's azimuthal angle with respect to the beam axis distributions.

A.16 Plotted are the reconstructed leading proton angle with respect to the beam axis residual in the measured $\mathrm{Q}_{\mathrm{QE} \text {,proton }}^{2}$ bins. . . . . . . . . . 235

A.17 Plotted are the reconstructed leading proton momentum (left) and $\mathrm{Q}_{\mathrm{QE} \text {,proton }}^{2}$ (right) residuals. . . . . . . . . . . . . . . . . . . . 236

A.18 Plotted are the area (top) and absolutely (bottom) normalized reconstructed leading proton momentum. . . . . . . . . . . . . 237

A.19 Plotted are the reconstructed $\mathrm{Q}_{\mathrm{QE} \text {,proton }}^{2}$ residual in the measured $\mathrm{Q}_{\mathrm{QE} \text {,proton }}^{2}$

A.20 Plotted is the proton pID score after applying all of the analysis cuts which isolated the $\nu_{\mu}$ QE-like candidates. . . . . . . . . . . . 239

B.1 The reconstructed $\mathrm{Q}^{2}$ difference between the muon and proton kinematics, area(top) and absolutely(bottom) normalized data and Monte Carlo

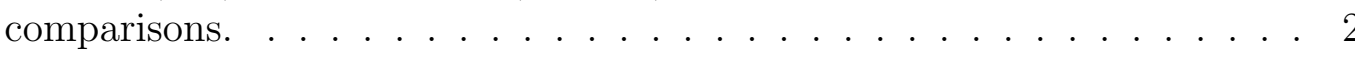

B.2 The area(top) and absolutely(bottom) normalized data and Monte Carlo comparisons of the proton transverse momentum. . . . . . . . . . . . . 242

B.3 The area(top) and absolutely(bottom) normalized data and Monte Carlo comparisons of the neutron transverse momentum. . . . . . . . . . . . 243

B.4 The differences between the projected and reconstructed proton angle for the area(top) and absolutely(bottom) normalized data and Monte Carlo comparisons. 
C.1 Plotted is the efficiency as function of $\mathrm{Q}_{\mathrm{QE} \text {,proton }}^{2}$ for the $\nu_{\mu}$ QE-like signal without (top) and with (bottom) the condition on the proton's momentum (at least one proton in the final state with momentum greater than $450 \mathrm{MeV} / \mathrm{c})$.

C.2 Plotted is the purity as function of $\mathrm{Q}_{\mathrm{QE} \text {,proton }}^{2}$ for the $\nu_{\mu}$ QE-like signal without (top) and with (bottom) the condition on the proton's momentum (at least one proton in the final state with momentum greater than $450 \mathrm{MeV} / \mathrm{c})$.

C.3 Plotted is the efficiency as function of the proton's momentum for the $\nu_{\mu}$ QE-like signal without (top) and with (bottom) the condition on the proton's momentum (at least one proton in the final state with momentum

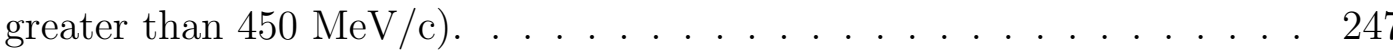

C.4 Plotted is the purity as function of the proton's momentum for the $\nu_{\mu}$ QE-like signal without (top) and with (bottom) the condition on the proton's momentum (at least one proton in the final state with momentum

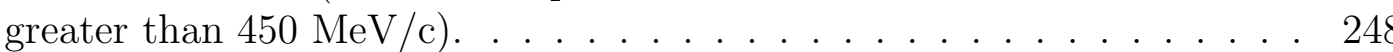

C.5 Plotted is the efficiency as function of the proton's polar angle for the $\nu_{\mu}$ QE-like signal without (top) and with (bottom) the condition on the proton's momentum (at least one proton in the final state with momentum greater than $450 \mathrm{MeV} / \mathrm{c}$ ). . . . . . . . . . . . . . . . . . 249

C.6 Plotted is the purity as function of the proton's polar angle for the $\nu_{\mu}$ QElike signal without (top) and with (bottom) the condition on the proton's momentum (at least one proton in the final state with momentum greater

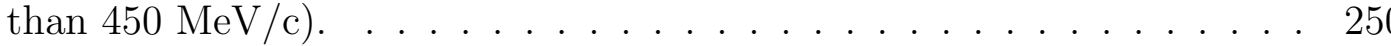




\section{SECTION 1 INTRODUCTION}

The complexity of the universe has always fascinated mankind. To understand the evolution of the universe, the behaviors and properties of elementary particles and the forces which govern them must also be understood. One of the ways in which particle and nuclear physics aim to uncover the mysteries of particles and the forces of nature is by bombarding nuclear matter with a beam of particles. For the last 50 years, nuclear physicists have extensively analyzed electron scattering as a tool for studying the atomic nucleus and its constituents. More recently, dedicated precision neutrino experiments have studied the neutrino collisions on nuclei with $\mathrm{A}>2$, whereas the majority of the previous neutrino experiments measured the neutrino cross sections on hydrogen and deuterium targets. The recent data have shown that there are nuclear physics beyond the independent nucleon impulse approximation which are not accurately modeled in the Monte Carlo codes modeling neutrino scattering. As a result, nuclear physicists are propagating the knowledge obtained from electron scattering to neutrino scattering.

Nevertheless, the information which can be extracted from studying the atomic nucleus and its constituents depends on the force that is exchanged through the interaction. Electrons primarily scatter by exchanging massless vector bosons called photons, $\gamma$, where a small contribution to the scattering amplitude appears from the exchange of the massive vector boson, $Z^{0}$. Neutrinos scatter through the exchange of the charge or neutral vector bosons, $W^{ \pm}$or $Z^{0}$. The $W^{ \pm}$and $Z^{0}$ bosons, the carriers of the weak nuclear force, violate parity symmetry (discussed later in the chapter), which leads to one major source of difference between the two different 
types of lepton scattering. As a result, there are properties of the nucleus that are less or more sensitive to neutrino scattering than electron scattering. For that reason, neutrino scattering plays an important and particular role in the understanding of nuclear physics. The neutrino scattering from a nucleon bound in a nucleus (neutrinonucleon cross section) is the focus of this dissertation.

One of the ways to characterize elastic scattering cross sections is by form factors, which represent the fundamental structure of the nucleon (see Chapter 2). The nucleon is a complex system and is described by various types of form factors, which depend on the neutrino interaction process and the kinematic phase space. The quasielastic interaction channel is the neutrino scattering process that is explored within this thesis. In neutrino scattering, the quasielastic interaction is defined as the process in which a neutrino scatters from a nucleon in the nucleus and via the exchanged of a $W^{ \pm}$boson, a charged lepton and nucleon are emitted. This reaction is sensitive to the axial form factor (see Subsection 2.2.5) and the free parameter of the axial form factor (under the dipole assumption) has been extracted by both the older and modern dedicated neutrino quasielastic cross section scattering experiments, where a summary of the results is given in Chapter 2. In short, the results show that the neutrino scattering from a nucleus with $\mathrm{A}>2$ is sensitive to nuclear dynamics such as meson exchange currents, which have been previously observed in electron-nucleus scattering data.

For scattering on a nucleon in a nucleus heavier than Hydrogen, the recoil nucleon can interact with the spectator nucleons before exiting the nucleus. This is known as a final state interaction (see Section 2.4). The effects of final state interactions (FSI) have been extensively studied in electron scattering experiments for a wide variety of nuclear targets. One of the goals for the current neutrino cross section experiments is to study the effects of FSI in neutrino scattering for $\mathrm{A}>2$. Note that several descriptive arguments that illustrate the critical need for analyzing FSI effects in 
neutrino scattering, are presented in Section 2.4. In short, these arguments advocate the importance of studying FSI effects by focusing on the critical need to understand FSI influence on the kinematic distributions of the ejected hadrons from the scattering process. Under the influence of FSI, hadrons may never even escape the nucleus. This has significant impact on the measured event rate for a particular neutrino interaction channel that an experiment may observe. Therefore, it is essential to have a specific description of the neutrino reaction channel which is being detected. This dissertation focuses on the effects of FSI for the muon neutrino quasielastic-like process, meaning that a muon and at least one proton are detected in the final state.

This chapter summarizes the historical overview of the neutrino, fundamental properties of the Standard Model and the weak nuclear force, and the motivation in neutrino particle physics that lead to this analysis. The last discussion point will explain the importance of neutrino cross section measurements for the current and near future neutrino oscillation experiments. A brief illustration of the remaining chapters in this dissertation is shown below.

- Chapter 2 gives an overview of the electron and neutrino scattering on both a free nucleon and those in the nucleus.

- Chapter 3 provides a description of the detector package (the MINER $\nu \mathrm{A}$ experiment) needed to produce precision cross section measurements for the neutrino-nucleus scattering.

- Chapter 4 outlines the detector calibration, simulation, and reconstruction of the events in the detector.

- Chapter 5 describes the method for selecting the $\nu_{\mu}$ charged current quasielasticlike candidates, along with a detail description of the systematic uncertainties.

- Chapter 6 concludes with the results which give an interpretation of the effects of final state interactions for the $\nu_{\mu}$ quasielastic-like cross section. 


\subsection{HISTORICAL OVERVIEW OF THE NEUTRINO}

The birth of modern nuclear physics emerged from Antonie Henri Becquerel's accidental discovery of radioactivity (radioactive decay): the process which an unstable atomic nucleus looses energy by emitting a particle(s) or the transformation of an atom into a different atom or into a lower energy state [3]. From the pioneering research of Becquerel's former doctoral student, Marie Curie, her husband, Pierre, and Ernest Rutherford, the three distinct forms of radiation were discovered: alpha ( $\alpha$-decay), beta $(\beta$-decay), and gamma ( $\gamma$-rays). Today, it is known that $\alpha$-particles are the bound states of two protons and two neutrons (the nucleus of the Helium atom), $\beta$-particles are electrons, and $\gamma$-rays are photons, the fundamental particle of light. To prevent the violation of the law of energy conservation for the $\beta$-decay, a neutral particle, the neutrino, is emitted along with the $\beta$ particle. The neutrino $(\nu)$ was first postulated by Wolfgang Pauli in 1930. However, Enrico Fermi developed the first theoretical explanation of the $\beta$-decay, and the theory, the four-point beta decay required the existence of the neutrino particle (see Figure 1.1). The theory showed that the neutron is a bound nucleon in the nucleus, and via the weak nuclear interaction, the neutron decays into a proton by simultaneously emitting an electron and a neutrino.

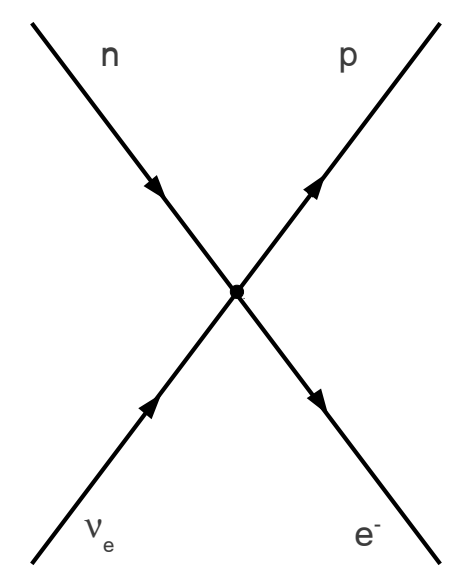

Figure 1.1. The Feynman diagram of Enrico Fermi's proposal of the four-point $\beta$-decay. 
Due to the chargeless property of the neutrino, its detection must be observed from the outgoing particles originating from the neutrino interaction with matter. Symmetry plays a fundamental role in particle physics. Under the time reversal symmetry, the inverse $\beta$-decay (see Figure 1.2) is a valid process, which occurs in nature. The signature of the inverse $\beta$-decay, $\nu_{e}\left(\bar{\nu}_{e}\right)+\mathrm{n}(\mathrm{p}) \rightarrow e^{\mp}+\mathrm{p}(\mathrm{n})$, must be detected in order to prove the existence of the neutrino.

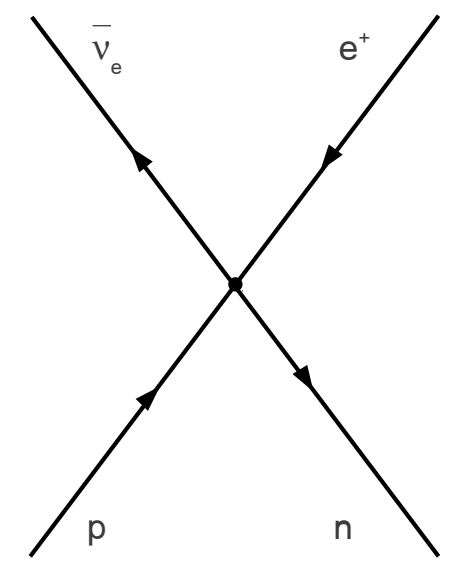

Figure 1.2. The Feynman diagram of the inverse $\beta$-decay. The arrows point backwards for the anti-particles, where anti-particles can be interpreted as moving backwards in time.

Along with developing detectors to observe the neutrino, physicists also were expanding Fermi's theory to understand the physical properties of the neutrino and the weak nuclear interaction. Inspired by the beautiful theory of Quantum Electrodynamics, the theorist, Hideki Yukawa, proposed the W gauge vector boson [3]. Based on Yukawa's theory of the nuclear force, the particles do not converge at an interaction point. Instead, the $\mathrm{W}$ boson is an intermediate particle of the decay, a particle which transmits the force between the nucleons. The Figure 1.3 shows the Feynman diagram of Yukawa's proposal of the gauge boson in the $\beta$-decay.

Arising from the efforts to understand the nuclear force, accelerators and detectors in both the fields of particle and astro-physics discovered a zoo of new particles. New theories emerged which more accurately distinguished the difference between 


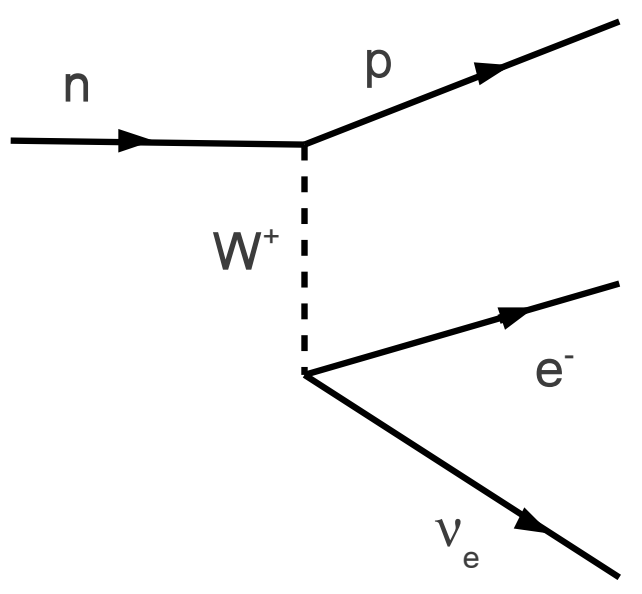

Figure 1.3. A Feynman diagram of H. Yukawa's proposal of the intermediate particle, W gauge boson, in the $\beta$-decay.

the fundamental forces, illustrated the weak nuclear interaction, and interpreted the nature of the zoo of particles. Eventually, the formulated theories were united in the Standard Model.

\subsection{THE QUARK FORMALISM IN THE STANDARD MODEL}

The various combination of particles called quarks, shown in the Table 1.2, make up the zoo of composite particles observed in accelerator experiments.

Table 1.1. The Quark Family of spin $=\frac{1}{2}$.

\begin{tabular}{|l||l|l|l|}
\hline $\mathrm{Q}=+\frac{2}{3} e$ & up & charm & top \\
$\mathrm{Q}=-\frac{1}{3} e$ & down & strange & bottom \\
\hline
\end{tabular}

Today, it is known that the quarks and leptons (Table 1.2) are the elementary (pointlike) particles of nature and the fundamental forces, that allow the particles to interact with each other are the electromagnetic, weak nuclear, and strong nuclear forces. 
Gravity is also a fundamental force, but it can be ignored due to its extremely weak nature. The Standard Model is the theoretical framework which unites the forces and is guided by the underlying existence of the various symmetries in the nature of particles.

Table 1.2. The Lepton Family of spin $=\frac{1}{2}$.

\begin{tabular}{|l|l|l|}
\hline electron $(\mathbf{e})$ & muon $(\mu)$ & $\operatorname{tau}(\tau)$ \\
electron-neutrino $\left(\nu_{e}\right)$ & muon-neutrino $\left(\nu_{\mu}\right)$ & tau-neutrino $\left(\nu_{\tau}\right)$ \\
\hline
\end{tabular}

The baryons are particles composed of the three quarks (qqq) or anti-quarks $(\overline{q q q})$ bound states and mesons $(\mathrm{q} \overline{\mathrm{q}})$ are a quark-antiquark pair. Other configurations of a quark system violate a fundamental law of Quantum Chromodynamics (QCD), color conservation. One of the original motivations for the introduction of the color quantum number was due to the existence of the $\Delta^{++}$(uuu) particle. The $\Delta^{++}$

baryon has three identical spin $\frac{1}{2}$ fermions in the ground state. A state in this configuration is forbidden under the Pauli exclusion principle. The u-quarks in the $\Delta^{++}$ ground state come in three primary colors: red $(R)$, green $(G)$, and blue $(B)$. The color quantum number is responsible for making the u-quarks distinguishable. All hadrons are observed as colorless particles, meaning that they are invariant under color transformations.

\section{Color Composition of Hadrons}

- Three quarks system (baryons): Equal mixture of R, G, B,

- Three antiquarks system (anti-baryons): Equal mixture of $\overline{\mathrm{R}}, \overline{\mathrm{G}}, \overline{\mathrm{B}}$,

- Quark-antiquark pair (mesons): Equal mixture of $R \bar{R}, G \bar{G}, B \bar{B}$.

\subsubsection{ADDITIONAL PROPERTIES OF QUARKS}

The additive quantum number of the quarks must be conserved. The additive quantum number of the quark system is known as the hypercharge, 


$$
\mathrm{Y} \equiv \mathrm{B}+\mathrm{S} .
$$

Here $\mathrm{Y}, \mathrm{B}$, and $\mathrm{S}$ are the hypercharge, baryon, and strange quantum numbers, respectively. Each quark is assigned a baryon number, $B=\frac{1}{3}$. The up and down quarks are assigned a strangeness, $\mathrm{S}=0$ and the strange quark has a strangeness, $\mathrm{S}=1$. The hypercharge has no physical interpretation, but is a property of the $S U(3)$ multiplet. The $S U(3)$ multiplet is discussed later in this section. The hypercharge is related to the charge of the particle,

$$
\mathrm{Q}=\mathrm{I}_{z}+\frac{Y}{2},
$$

where $\mathrm{Q}$ is the charge and $\mathrm{I}_{z}$ is the projection of the isospin along the $\mathrm{z}$-axis. The isospin is described in the next paragraph.

The neutron and proton are baryons with nearly identical mass, but different charges. The nucleons' quark flavor composition accounts for the difference in the total charge. The proton has two up quarks and one down quark giving a total charge of $2\left(\frac{2}{3}\right) e-\frac{1}{3} e=+1 e$ and the neutron has two down quarks and one up quark for a total charge of $\frac{2}{3} e-2\left(\frac{1}{3}\right) e=0$. Based on the mass of the nucleons, under isospin symmetry, the neutron and proton are interpreted as different quantum states of the same particle: spin up and spin down nucleon-nucleon system. Isospin is not a spin observable, however, the mathematical structure of spin is used to describe its properties. Therefore, the isospin eigenstate $\left|I I_{3}\right\rangle$ of spin up $(\uparrow)$ and spin down $(\downarrow)$ nucleons are:

$$
\begin{aligned}
& \text { proton }=\left|\frac{1}{2} \frac{1}{2}\right\rangle \\
& \text { neutron }=\left|\frac{1}{2}-\frac{1}{2}\right\rangle
\end{aligned}
$$

Additionally, the strong nuclear force, the force which binds the nucleons in the nucleus, is invariant under isospin transformation. The strong nuclear force interacts the same for each nucleon, in spite of the state's isospin projection. 
Motivated by the wave of new particles invading the world of particle physics, Gell-Mann extended the concept of the nucleon mass or isospin symmetry ( $S U$ (2) doublet) to the quarks with the Eightfold-Way [4]. The nucleons and all of the other baryons that are built from the different compositions of the lightest quarks $(\mathrm{u}, \mathrm{d}$, and s) form a flavor symmetry, the $S U(3)$ symmetry. The hadrons that contain a strange quark(s) are heavier than all others, thus the flavor $S U(3)$ triplet is not an exact symmetry. In spite of the mass symmetry breaking, the flavor $S U(3)$ symmetry is very useful for generalizing the various hardonic states.

The three-quark system forms the following multiplets:

$$
\begin{aligned}
& \text { mesons }(q \bar{q}): 3 \bigotimes \overline{3}=8 \bigoplus 1 \\
& \text { baryons (qqq) : } 3 \bigotimes 3 \bigotimes 3=10 \bigoplus 8 \bigoplus 8 \bigoplus 1
\end{aligned}
$$

The decomposition of the three-quark triplets show that the lightest baryons in the quark model are characterized by a spin $=\frac{3}{2}$ decuplet and spin $=\frac{1}{2}$ octet. The decuplet consists of ten symmetric flavor states. In fact, the $\Delta^{++}$baryon falls within the decuplet. As mentioned previously, the three-quark states are fermions, therefore, the total wavefunction must be antisymmetric. This is the original motivation for the quark color conservation that was described earlier. As a result, the quarks form an exact color $S U(3)$ symmetry. The orbital-spin-flavor-color states represent the total wavefunction of the three-quark system. The octets are built from eight mixed symmetric states, where the states can either be symmetric or antisymmetric under the interchange of the first two quarks. The proton and neutron are members of the octet multiplet.

In the early quark model, the proton and neutron are composed of three valence quarks and the strong nuclear force binds the quark constituents in the nucleon. The free neutron, the heavier nucleon, is an unstable baryon and decays via the weak nuclear force. Fermi's theory shows that the weak interaction changes the neutron (udd) into a proton (uud). Not only does the $\beta$-decay give rise to the existence of the 
neutrino (the neutrino is needed to ensure energy conservation), it reveals that both the quarks and leptons interact via the weak nuclear force, as indicated in Figure 1.4.

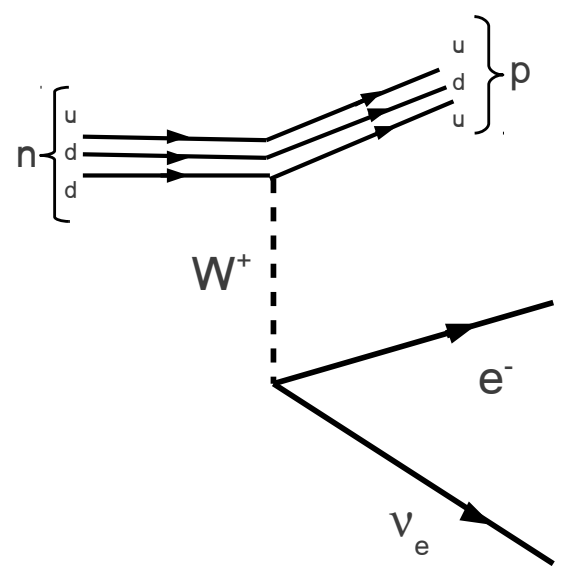

Figure 1.4. The Feynman diagram of the quark model of the $\beta$-decay.

\subsection{THE WEAK NUCLEAR INTERACTION}

It was observed that there are particles which can decay via the electromagnetic interaction in about $10^{-16} \sec \left(\right.$ for example, $\pi^{0} \rightarrow \gamma \gamma$ ) and strong nuclear interaction in about $10^{-23} \sec \left(\right.$ for example, $\Delta^{++} \rightarrow \mathrm{p}+\pi^{+}$). However, the free neutron has a mean lifetime of about $882 \mathrm{sec}$. The lifetimes are inversely related to a non-dimensional quality, the coupling constant. The coupling constant describes the strength of the force exerted in an interaction. The weak interaction was proposed long before the discover of the pion and delta-baryon, but still their decays are additional evidence of an interaction with a weaker coupling constant $\left(\alpha_{W} \ll \alpha_{S}<\alpha\right)$. Here $\alpha_{W}$ is the weak coupling constant, $\alpha_{S}$ is the strong coupling constant, and $\alpha$ is the electromagnetic coupling constant, which is known as the fine-structure constant. The fine-structure constant is discussed in the next chapter. 
The starting point for calculating the decay rates is Fermi's Golden Rule, which describes the transition rate from the system initial state $|i\rangle$ to a final state $|f\rangle$ as

$$
W=\frac{2 \pi}{\hbar}\left|M_{f i}\right|^{2} \rho\left(E_{f}\right)
$$

Here, the terms, $\rho\left(\mathrm{E}_{f}\right)$ is the density of the final state and $\mathrm{M}_{f i}$ is the time-dependent perturbation matrix element between the initial and final states of a system,

$$
M_{f i}=\left\langle\psi_{f}(t)\left|H^{\prime}(t)\right| \psi_{i}(t)\right\rangle
$$

The $\beta$-decay in Figure 1.1 is modified to show the weak currents in Figure 1.5. The matrix element, Lorentz invariant amplitude, for the $\beta$-decay is also written as,

$$
\begin{aligned}
\left|M_{f i}\right| & =G_{F}\left(\bar{u}_{n} \gamma^{\mu} u_{p}\right)\left(\bar{u}_{\nu_{e}} \gamma_{\nu} u_{e}\right) \\
& =G_{F}\left(j^{\mu} j_{\nu}\right) .
\end{aligned}
$$

The term $\mathrm{G}_{F}$ has dimensions and is called the Fermi's coupling constant with the value of $1.166 \times 10^{-5} \mathrm{GeV}^{-2}$. In Fermi's theory, the $\beta$-decay is described using theory of the four-point $\beta$-decay.

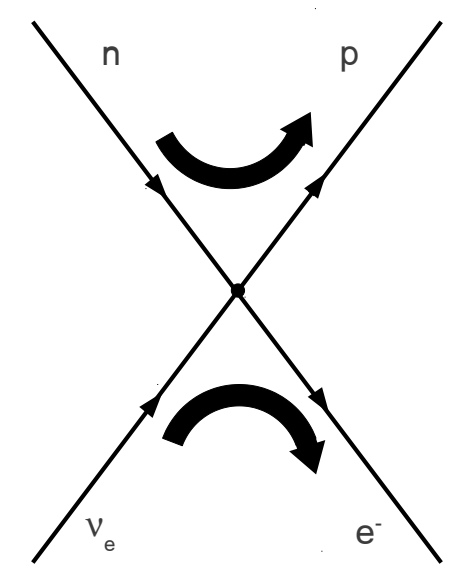

Figure 1.5. The Feynman diagram of the four-point $\beta$-decay where the arrows represent the structure of the weak currents at the hadronic and leptonic vertices. 
Fermi's construction of a vector-vector current to describe the invariant amplitude, $\left|\mathrm{M}_{f i}\right|$, is a very good approximation for explaining some of the properties of the weak interaction. As mentioned earlier, the concept of symmetry is utilized to verify if a system is invariant under rotations. It is expected that all physical systems should be conserved under the parity and charge conjugation transformations. The parity operates on the wavefunction and rotates the space-coordinates around an axis perpendicular to the, $P \psi(x, y, z, t)=\psi(-x,-y,-z, t)$, plane. In other words, under parity conservation, the system is invariant when viewing its mirror image.

Parity was proposed first to be violated in the kaon systems by theoretical physicists, Tsung-Dao Lee and Chen-Ning Yang in 1956 [4]. Immediately following the discovered of parity violation for neutral kaons, one of the greatest experimentists, Chien Shiung Wu (commonly known as Madame Wu) lead a team to futher investagate the conservation of parity. The $\mathrm{Wu}$ experiment, the nuclear $\beta$-decay experiment of the polarized cobalt nuclei, ${ }^{60} \mathrm{Co} \rightarrow{ }^{60} \mathrm{Ni}^{*}+\mathrm{e}^{-}+\bar{\nu}_{e}$, also confirmed and provided additional support that parity is not conserved in the weak interaction . Parity reverses the momentum of the particle while the spin angular momentum remains unchanged. The data from the ${ }^{60}$ Co experiment showed that for the majority of the events, the electron is emitted anti-parallel to the ${ }^{60} \mathrm{Ni}^{*}$ nuclear spin vector. If parity is conserved, then approximately the same amount of events with the electron emitted in parallel and anti-parallel directions should of have been observed.

The weak interaction is not conserved under charge symmetry as well. The charge conjugation operator transforms the particle into an anti-particle and vice versa. The positive charged pion decays as, $\pi^{+} \rightarrow \mu^{+}+\nu_{\mu}$. The neutrino in the $\pi^{+}$decay is always emitted with a left-handed helicity as determined by experiments. The negative charged pion decay transforms as, $\pi^{-} \rightarrow \mu^{-}+\bar{\nu}_{\mu}$ and the anti-neutrino emitted is always right-handed. If charge is conserved in the pion decay, then antineutrinos emitted in the $\pi^{-}$decay should have a left-handed helicity. When charge 
and parity are combined, the operator CP transforms the left-handed neutrino into the right-handed neutrino. The $\mathrm{CP}$ is conserved in the pion decay. However, the weak interaction is only $97 \%$ invariant under the CP symmetry.

Because the weak interaction violates parity, the vector current, $\gamma^{\mu}$, is replaced by a vector-axial vector current $(\mathrm{V}-\mathrm{A}), \gamma^{\mu}\left(1-\gamma^{5}\right)$, such that the weak interaction remains invariant under the $\mathrm{CP}$ operation. Then the Equation 1.3.3 is rewritten as,

$$
\begin{aligned}
\left|M_{f i}\right| & =\frac{G_{F}}{\sqrt{2}}\left[\bar{u}_{n} \gamma^{\mu} \frac{1}{2}\left(1-\gamma^{5}\right) u_{p}\right]\left[\bar{u}_{\nu_{e}} \gamma_{\nu} \frac{1}{2}\left(1-\gamma^{5}\right) u_{e}\right] \\
& =\frac{G_{F}}{\sqrt{2}}\left[\bar{u}_{n} \gamma^{\mu} \frac{1}{2}\left(1-\gamma^{5}\right) u_{p}\right]\left[\bar{u}_{e} \gamma_{\nu} \frac{1}{2}\left(1-\gamma^{5}\right) u_{\nu_{e}}\right] \\
& =\frac{4 G_{F}}{\sqrt{2}}\left(j^{\mu} j_{\nu}^{\dagger}\right) .
\end{aligned}
$$

The $1 / \sqrt{2}$ term is needed to keep the original definition of the $\mathrm{G}_{F}$ with the value of $1.166 \times 10^{-5} \mathrm{GeV}^{-2}$. The factor 4 comes into play because the normalized projection operator $\frac{1}{2}\left(1-\gamma^{5}\right)$ is used. The strength of the coupling constant $\mathrm{G}_{F}$ has been measured from nuclear $\beta$-decay experiments. $\mathrm{G}_{F}$ can be redefined as an universal constant which describes the strength of the weak interaction for all processes.

The electromagnetic electron-proton elastic scattering is Fermi's inspiration for the four-point $\beta$-decay. Thus, $\mathrm{G}_{F}$ is the analogy of $e^{2} / q^{2}$. In addition, the nuclear $\beta$ decay invariant amplitude is modified to incorporate a gauge boson field. For example, the invariant amplitude for the muon decay $\left(\mu^{-} \rightarrow \nu_{\mu} \mathrm{e}^{-} \nu_{e}\right)$ is written as,

$$
\left|M_{f i}\right|=\left(\frac{g_{W}}{\sqrt{2}} \bar{u}_{\nu_{\mu}} \gamma^{\sigma} \frac{1}{2}\left(1-\gamma^{5}\right) u_{\mu}\right) \frac{1}{M_{W}^{2}-q^{2}}\left(\frac{g_{W}}{\sqrt{2}} \bar{u}_{e} \gamma_{\sigma} \frac{1}{2}\left(1-\gamma^{5}\right) u_{\nu_{e}}\right) .
$$

Here, $\mathrm{M}_{W}$ is the mass of the gauge $\mathrm{W}$ boson (a carrier of the weak nuclear force) and the weak coupling constant, $\alpha_{W}=\mathrm{g}_{W} / \sqrt{2}$, is dimensionless. In the limit, where the four-momentum $\left(\mathrm{q}^{2}\right)$ transferred by the weak boson is much smaller than the mass of the boson squared, the weak currents interact at a point (the four-point $\beta$-decay). 


$$
\frac{G_{F}}{\sqrt{2}}=\frac{g_{W}^{2}}{8 M_{W}^{2}}
$$

Motivated by Maxwell's theory of electromagnetism, the unification of electricity and magnetism, Sheldon Galasow, Abdus Salam, and Steven Weinberg, unified the electromagnetic and weak nuclear interactions into the electroweak theory in the 1960s [5]. The electroweak theory predicted the existence of the $\mathrm{Z}^{0}$, the neutral current gauge boson. Until 1973 all of the observed weak interactions were consistent under the hypothesis that the weak nuclear force is mediated by the exchange of the charged current gauge bosons, $\mathrm{W}^{ \pm}$. Then, the data from the Gargamelle bubble chamber experiment observed reactions of the type $\nu_{\mu}+\mathrm{N} \rightarrow \nu_{\mu}+\mathrm{X}[5]$. These neutral currents processes confirmed that the weak interaction does mediate via a neutral current gauge boson.

The first experimental evidence of the carriers of the weak nuclear force, $\mathrm{W}^{ \pm}$and $\mathrm{Z}^{0}$ bosons, were observed by the Super Proton Synchrotron accelerator in 1983 at the European Organization for Nuclear Research (CERN) via antiproton and proton collisions [5].

$$
\begin{aligned}
& \bar{p}+p \rightarrow W^{ \pm}+X^{\mp} \\
& \bar{p}+p \rightarrow Z^{0}+X^{0}
\end{aligned}
$$

The gauge bosons are not detected directly, but observed from their decay products:

$$
\begin{gathered}
W^{+} \rightarrow l^{+}+\nu_{l} \\
W^{-} \rightarrow l^{-}+\bar{\nu}_{l} \\
Z^{0} \rightarrow l^{+}+l^{-} \\
Z^{0} \rightarrow \nu_{l}+\bar{\nu}_{l}
\end{gathered}
$$


The $\mathrm{W}^{ \pm}$and $\mathrm{Z}^{0}$ bosons are massive with masses measured of $\mathrm{M}_{W}=80.6 \mathrm{GeV} / \mathrm{c}^{2}$ and $\mathrm{M}_{Z}=91.2 \mathrm{GeV} / \mathrm{c}^{2}$, which is unlike the massless photon, that carries the electromagnetic force.

The phenomenon that allows nature to produce particle reactions via the weak neutral currents, gives rise to the symmetry between the weak nuclear and electromagnetic forces. The actuality that both the leptons and quarks are able to participate in the weak interaction via the $\mathrm{V}$-A charged currents, introduces the lepton-quark symmetry.

As shown earlier in the Tables 1.2 and 1.2, the Standard Model predicts three generations of lepton and quark families. The first two generations of quarks are

$$
\left(\begin{array}{l}
u \\
d
\end{array}\right) \text { and }\left(\begin{array}{l}
c \\
s
\end{array}\right)
$$

and the first two generations of leptons are

$$
\left(\begin{array}{c}
\nu_{e} \\
e^{-}
\end{array}\right) \text {and }\left(\begin{array}{c}
\nu_{\mu} \\
\mu^{-}
\end{array}\right) .
$$

Under the lepton-quark symmetry, the quarks and leptons are assumed to have identical weak interactions. As indicated in the Equations 1.3.8, the $\mathrm{W}$ bosons can decay into leptons. The lepton-quark symmetry implies that the leptons can be replaced by the quarks in the $\mathrm{W}^{ \pm}$vertices as illustrated in the Figure 1.6. The symmetry suggests that a process such as $\mathrm{d}+\bar{u} \rightarrow \mathrm{W}^{-}$has the same coupling as the corresponding leptonic process as displayed in the Equation 1.3.8.

The naive assumption of the lepton-quark symmetry describes many particle reactions. However, the kaon decay $\left(\mathrm{K}^{-} \rightarrow \mu^{-}+\bar{\nu}_{\mu}\right)$ is forbidden under this symmetry. The $\mathrm{K}^{-}$requires the existence of the $\mathrm{s}+\bar{u} \rightarrow \mathrm{W}^{-}$vertex and the Figure 1.6 shows that the process does not exist within this naive picture of the lepton-quark symmetry. Due to the flavor quark symmetry breaking in the kaon system, the lepton-quark symmetry is extended to incorporate the mixing between the quark's states (quark 


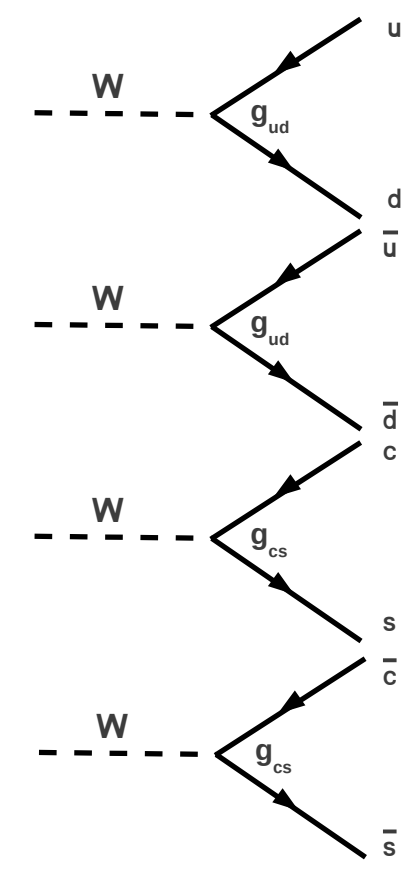

Figure 1.6. The $\mathrm{W}^{ \pm}$quark vertices for lepton-quark symmetry, excluding the quark mixing.

mixing). The originally proposal of the lepton-quark symmetry assumes no oscillations between the quarks. To account for the quark mixing, the first two generations of quarks are rewritten as

$$
\left(\begin{array}{c}
u \\
d^{\prime}
\end{array}\right) \text { and }\left(\begin{array}{c}
c \\
s^{\prime}
\end{array}\right)
$$

where $\left|d^{\prime}\right\rangle$ and $\left|s^{\prime}\right\rangle$ are the linear combination of $|d\rangle$ and $|s\rangle$ quark states,

$$
\begin{aligned}
& d^{\prime}=d \cos \theta_{C}+s \sin \theta_{C} \\
& s^{\prime}=-d \sin \theta_{C}+s \sin \theta_{C},
\end{aligned}
$$

and the parameter $\theta_{C}$ is the Cabibbo angle, also known as the quark mixing angle. The $\mathrm{W}$ vertex, $\mathrm{u}+\bar{s} \rightarrow \mathrm{W}^{-}$, is allowed as illustrated in the Figure 1.7. Now, the kaon decay, $\mathrm{K}^{-} \rightarrow \mu^{-}+\bar{\nu}_{\mu}$ is described by the lepton-quark symmetry. 


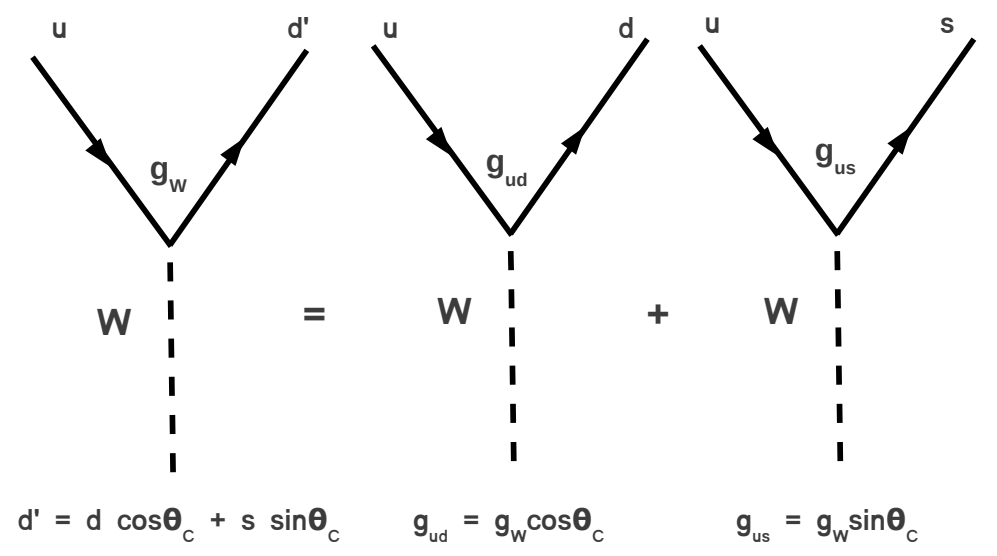

Figure 1.7. The quark mixing interpretation of the $u+d^{\prime} \rightarrow W$ vertex.

The mixing of quark states was introduced by Nicola Cabibbo in order to explain the weak decay of the strange particles. Historically, the most exciting development from the theory of quark-mixing was the prediction of the charm quark. About ten years after the theoretical prediction, the charm quark was discovered at the Standard Linear Accelerator Center (SLAC), by a team of scientists led by Burton Richter. As mentioned previously, the Standard Model predicts three generations of leptons and quarks. When the third generation is taken into account, the $2 \times 2$ unitary mixing matrix becomes a $3 \times 3$ unitary mixing matrix, the CKM matrix. The CKM matrix is named after Cabibbo, Kobayashi and Maskawa, where Kobayashi and Maskawa extended the scheme to include the three generations of quarks.

\subsection{NEUTRINO OSCILLATIONS}

The knowledge obtained about the weak nuclear force from both the experiments and theories, since Fermi formulated the four point $\beta$-decay theory, is just astounding. Wolfgang Pauli would probably be amazed to learn that three generations of neutrinos 
exist. However, the physics of the Standard Model does not explained mother nature's entire story. The Standard Model assumes that the three flavor of neutrinos, $\nu_{e}$, $\nu_{\mu}$, and $\nu_{\tau}$, are massless particles. In addition, the three generations of leptons are essentially decoupled from each other. This means that the lepton quantum numbers, $L_{e}, L_{\mu}, L_{\tau}, L_{\nu_{e}}, L_{\nu_{\mu}}$, and $L_{\nu_{\tau}}$, are independently conserved. For example, the weak neutral and charged current reactions, such as $\nu_{e} \rightarrow \nu_{e}$ and $\nu_{e} \rightarrow \mathrm{e}^{-}$are permitted, whereas, the transitions such as $\nu_{\mu} \rightarrow \nu_{\tau}$ and $\nu_{e} \rightarrow \mu^{-}$are forbidden.

In 1961, Raymond Davis, Jr. led a team to detect the $\nu_{e}$, solar neutrinos produced in the Sun. The Homestake Experiment did not measure the expected number of solar neutrinos computed by the Standard Solar Model. The solar neutrino problem remained a mystery until about 1998. From the observation of atmospheric neutrinos, the Super-Kam $\ddot{i}$ okande experiment confirmed that neutrinos oscillate between flavors. This means that the electron neutrinos produced in the Sun changed to muon neutrinos along the way to Earth, therefore unobserved by the Homestake detector. The strong evidence of neutrino oscillations verifies that neutrinos are not massless particles. Thus, beyond the Standard Model, the neutrino is expected to have a small but finite mass.

The mathematical technique the lead to the CKM matrix $(3 \times 3$ quark mixing matrix), is used to build a $3 \times 3$ unitary matrix (Pontecorvo-Maki-Nakagawa-Sakata matrix) which connects the flavor eigenstates, $\nu_{e}, \nu_{\mu}$, and $\nu_{\tau}$ with the mass eigenstates, $\nu_{1}, \nu_{2}$, and $\nu_{3}$ (with masses $m_{1}, m_{2}$, and $m_{3}$ ) as shown in Equation 1.4.1.

$$
\left(\begin{array}{c}
\nu_{e} \\
\nu_{\mu} \\
\nu_{\tau}
\end{array}\right)=\left(\begin{array}{ccc}
U_{e 1} & U_{e 2} & U_{e 3} \\
U_{\mu 1} & U_{\mu 2} & U_{\mu 3} \\
U_{\tau 1} & U_{\tau 2} & U_{\tau 3}
\end{array}\right)\left(\begin{array}{c}
\nu_{1} \\
\nu_{2} \\
\nu_{3}
\end{array}\right)
$$

The PMNS unitary $3 \times 3$ matrix is displayed in the Equation 1.4 .2 , where $\mathrm{s}_{i j} \equiv \sin \theta_{i j}$, $\mathrm{c}_{i j} \equiv \cos \theta_{i j}:$ 


$$
\left(\begin{array}{ccc}
c_{12} c_{13} & s_{12} c_{13} & s_{13} e^{-i \delta} \\
-s_{12} c_{23}-c_{12} s_{23} s_{13} e^{i \delta} & c_{12} c_{23}-s_{12} s_{23} s_{13} e^{i \delta} & s_{23} c_{13} \\
s_{12} s_{23}-c_{12} c_{23} s_{13} e^{i \delta} & -c_{12} s_{23}-s_{12} c_{23} s_{13} e^{i \delta} & c_{23} c_{13}
\end{array}\right)
$$

The PMNS matrix is a representation of the product of three rotation matrices about the angles, $\theta_{12}, \theta_{23}$, and $\theta_{13}$ and a complex phase factor $\mathrm{e}^{i \delta}$, where $\delta$ is the CP-violating Dirac phase factor. The rotation of the neutrino mass eigenstates about the PMNS matrix gives the probability that the neutrino will be produced as one flavor but detected as a different flavor. The probability for the neutrino flavor conversion, such as $\nu_{e} \rightarrow \nu_{\mu}$ is written as

$$
\begin{aligned}
P\left(\nu_{e} \rightarrow \nu_{\mu}\right) & =\left|U_{e 1} U_{\mu 1}^{*} e^{-i E_{1} t}+U_{e 2} U_{\mu 2}^{*} e^{-i E_{2} t}+U_{e 3} U_{\mu 3}^{*} e^{-i E_{3} t}\right|^{2} \\
P\left(\nu_{e} \rightarrow \nu_{\mu}\right) & =2 \operatorname{Re}\left(U_{e 1} U_{\mu 1}^{*} U_{e 2} U_{\mu 2}^{*}\left[e^{-i\left(E_{1}-E_{2}\right) t}-1\right]\right) \\
& +2 \operatorname{Re}\left(U_{e 1} U_{\mu 1}^{*} U_{e 3} U_{\mu 3}^{*}\left[e^{-i\left(E_{1}-E_{3}\right) t}-1\right]\right) \\
& +2 \operatorname{Re}\left(U_{e 2} U_{\mu 2}^{*} U_{e 3} U_{\mu 3}^{*}\left[e^{-i\left(E_{2}-E_{3}\right) t}-1\right]\right)
\end{aligned}
$$

Here, $\mathrm{e}^{-i E_{i} t}$ is shorthand for $\mathrm{e}^{i p_{i} x-i E_{i} t}$. The same expression is written for the conversion probability, $\mathrm{P}\left(\nu_{\mu} \rightarrow \nu_{e}\right)[6]$. Unless the PMNS matrix elements are purely real, the results show that $\mathrm{P}\left(\nu_{e} \rightarrow \nu_{\mu}\right) \neq \mathrm{P}\left(\nu_{\mu} \rightarrow \nu_{e}\right)$. This means that if the PMNS matrix elements are complex, then the time reversal $(\mathrm{t} \rightarrow-\mathrm{t})$ symmetry is violated in neutrino oscillations. As mentioned previously, under the parity and charge operations (CP), left-handed neutrinos are converted into right-handed neutrinos and vice versa. In addition, the weak interaction does violate $\mathrm{CP}$ symmetry, which is demonstrated in the kaon decay experiments. If CP violation exists in the neutrino sector, this implies that the PMNS matrix elements are complex as discovered in the quark sector. However, the weak interaction is conserved under charge, parity, and time reversal $(\mathrm{CPT})$ symmetry, and therefore, $\mathrm{P}\left(\bar{\nu}_{e} \rightarrow \bar{\nu}_{\mu}\right)=\mathrm{P}\left(\nu_{\mu} \rightarrow \nu_{e}\right)$. If the possibility of $\mathrm{CP}$ violation is neglected, then the Equation 1.4.3 becomes, 


$$
\begin{aligned}
P\left(\nu_{e} \rightarrow \nu_{\mu}\right) & =2 U_{e 1} U_{\mu 1} U_{e 2} U_{\mu 2} R e\left[e^{-i\left(E_{1}-E_{2}\right) t}-1\right] \\
& +2 U_{e 1} U_{\mu 1} U_{e 3} U_{\mu 3} \operatorname{Re}\left[e^{-i\left(E_{1}-E_{3}\right) t}-1\right] \\
& +2 U_{e 2} U_{\mu 2} U_{e 3} U_{\mu 3} \operatorname{Re}\left[e^{-i\left(E_{2}-E_{3}\right) t}-1\right] \\
P\left(\nu_{e} \rightarrow \nu_{\mu}\right)= & -4 U_{e 1} U_{\mu 1} U_{e 2} U_{\mu 2} \sin ^{2} \Delta_{12} \\
& -4 U_{e 1} U_{\mu 1} U_{e 3} U_{\mu 3} \sin ^{2} \Delta_{13} \\
& -4 U_{e 2} U_{\mu 2} U_{e 3} U_{\mu 3} \sin ^{2} \Delta_{23},
\end{aligned}
$$

where, $\Delta_{i j} \equiv \frac{\left(m_{i}^{2}-m_{j}^{2}\right) L}{4 E}=\frac{\Delta m_{i j}^{2} L}{4 E}$. Here, $m_{i}^{2}-m_{j}^{2}$ is the mass difference between the neutrino mass eigenstates, $\mathrm{L}$ is the distance of propagation, and $\mathrm{E}$ is the energy of the neutrino. The frequencies of the neutrino oscillation are determined by the neutrino mass differences,

$$
\begin{aligned}
& \Delta m_{21}^{2}=m_{2}^{2}-m_{1}^{2} \\
& \Delta m_{32}^{2}=m_{3}^{2}-m_{2}^{2} .
\end{aligned}
$$

The wavelength of the neutrino oscillations is, $\lambda=\frac{4 \pi E}{\Delta m_{i j}^{2}}$, where the $\lambda \sim \frac{1}{f}$ and $f$ is defined as the frequency, which is determined by the data [7] and [8]. In the scheme of the mass splitting,

$$
\left|\Delta m_{12}^{2}\right| \ll\left|\Delta m_{23}^{2}\right| \approx\left|\Delta m_{13}^{2}\right|
$$

the two different mass-squared terms are presented in the following convention as shown in the Figure 1.8.

In spite of which mass hierarchy is being consider, the convention in the Equation 1.4.5 leads to $\left|\Delta m_{13}\right| \approx\left|\Delta m_{23}\right|$. As a result and along with an unitary relation, the probability Equation 1.4.4 is simplified to,

$$
P\left(\nu_{e} \rightarrow \nu_{\mu}\right) \approx-4 U_{e 1} U_{\mu 1} U_{e 2} U_{\mu 2} \sin ^{2} \Delta_{12}+4 U_{e 3} U_{\mu 3} \sin ^{2} \Delta_{23}
$$




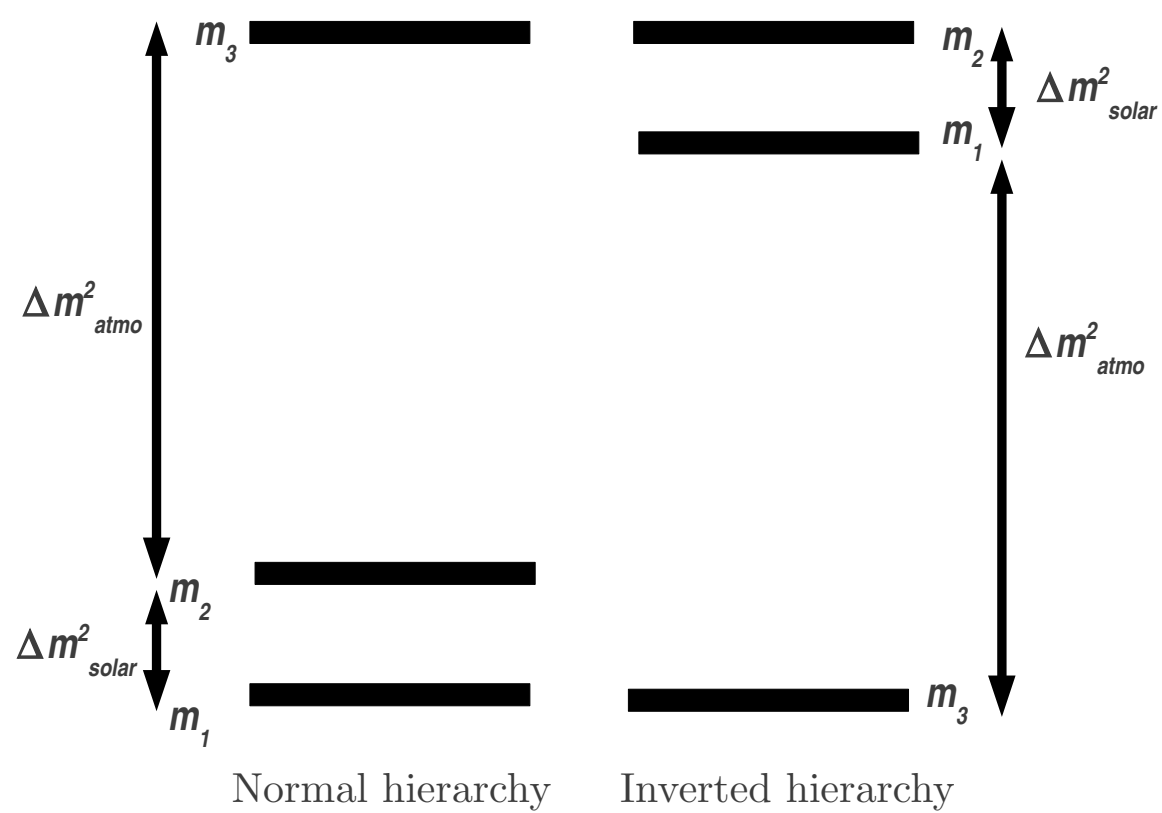

Figure 1.8. The hierarchical structure of the mass splitting in neutrino oscillations.

From the calculations and experimental measurements of the properties of atmospheric neutrinos, the term with the $\Delta_{12}$ can be neglected and the Equation 1.4.6 reduces to,

$$
P\left(\nu_{e} \rightarrow \nu_{\mu}\right) \approx 4 U_{e 3} U_{\mu 3} \sin ^{2} \Delta_{23}=4 \sin ^{2} \theta_{13} \sin ^{2} \theta_{23} \cos ^{2} \theta_{13} \sin ^{2} \Delta_{23}
$$

Similar equations can be written for the other conversion probabilities, $\mathrm{P}\left(\nu_{e} \rightarrow \nu_{\tau}\right)$ and $\mathrm{P}\left(\nu_{\mu} \rightarrow \nu_{\tau}\right)$ as well as the survival probabilities, $\mathrm{P}\left(\nu_{e} \rightarrow \nu_{e}\right), \mathrm{P}\left(\nu_{\mu} \rightarrow \nu_{\mu}\right)$, and $\mathrm{P}\left(\nu_{\tau} \rightarrow \nu_{\tau}\right)$.

There exists six independent parameters that govern neutrino oscillations, $\theta_{12}$, $\theta_{23}, \theta_{13}, \delta_{C P}, \Delta m_{21}^{2}$, and $\Delta m_{31}^{2}$. Since, the discovery of neutrino oscillation in 1998 , neutrino experiments have achieved great progress in measuring these parameters. 
The parameters $\theta_{12}$ and $\Delta m_{21}^{2}$ have been measured by solar and reactor neutrino experiments, $\theta_{23}$ and $\Delta m_{32}^{2}$ have been measured by atmospheric and accelerator experiments, and $\theta_{13}$ by the reactor neutrino and T2K experiments [8] and [9]. The CP-violating phase remains unknown. From the global analysis of the solar and KamLand experiments, the solar neutrinos parameters are found to be [10]

$$
\sin ^{2} \theta_{12}=0.304_{-0.016}^{+0.022}, \Delta m_{21}^{2}=7.65_{-0.20}^{+0.23} \times 10^{-5} \mathrm{eV}^{2}
$$

For the leading order atmospheric neutrinos parameters, $\theta_{23}$ and $\Delta m_{31}^{2}$, a global analysis of the atmospheric and MINOS experiments lead to a result of [10]

$$
\sin ^{2} \theta_{23}=0.50_{-0.06}^{+0.07},\left|\Delta m_{31}^{2}\right|=2.40_{-0.11}^{+0.12} \times 10^{-5} \mathrm{eV}^{2} .
$$

The Daya Bay experiment data shows that the neutrino mixing angle parameter, $\theta_{13}$ is [7] and [11]

$$
\sin ^{2} 2 \theta_{13}=0.089 \pm 0.010(\text { stat }) \pm 0.005 \text { (syst) }
$$

To measure the leptonic oscillation parameters, the neutrino oscillation experiments must calculate the neutrino event rate and energy distributions. Given that neutrinos interact via the weak nuclear force, neutrino detectors are designed large in size in order to observe a significant amount of events. In addition to the physical size of the detectors, the current and future neutrino experiments, such as the MINOS and $\mathrm{NO} \nu \mathrm{A}$ detectors, are composed of heavy atoms, such as Carbon and Iron [12], [13]. For just these reasons, it is indisputable that precision neutrino cross sections are necessary for extracting the neutrino oscillation parameters from neutrino oscillation experiments.

A concrete example of the importance of precision neutrino cross section measurements is presented by the $\mathrm{T} 2 \mathrm{~K}$ experiment [14]. For $\sin 2 \theta_{13}=0.1$, the $\mathrm{T} 2 \mathrm{~K}$ experiment reported a 0.105 uncertainty on the cross section. Reducing the uncertainty on the cross section is critical, particularly for extracting the unknown leptonic 
oscillation parameter, the CP-violating phase factor. This dissertation aims to provide information on what is needed in the neutrino event generators to reduce the uncertainties and how to improve the unknowns of neutrino interactions with heavy nuclei for the quasielastic cross section. The quasielastic channel is known as the standard candle for neutrino oscillation experiments. The neutrino event generator which models the neutrino interactions in this analysis, also is used by the current and future precision neutrino oscillation experiments. 


\section{SECTION 2}

\section{NEUTRINO CHARGED CURRENT QUASI-ELASTIC SCATTERING}

\subsection{INTRODUCTION TO ELASTIC SCATTERING}

Since the breakthrough results obtained from the Rutherford scattering experiment, the scattering of $\alpha$-particles off of a gold foil, scattering physics has been experimenters greatest tool for revealing the structure of nucleons and nuclei. One of the most fundamental types of scattering is the lepton-nucleon elastic collision,

$$
\mathrm{e}^{-}+\mathrm{N} \rightarrow \mathrm{e}^{-}+\mathrm{N}
$$

In this reaction the struck nucleon does not break apart and remains in its ground state. The electron elastic scattering is a effective technique that has been extensively utilized for examining and understanding the structure of the nucleon. Figure 2.1 shows the leading-order QED Feynman diagram for electron-nucleon scattering. In this process, a single virtual photon (the carrier of the electromagnetic interaction) is used to probe the structure of the nucleon. Recall that unlike the vector gauge bosons of the weak interaction, the photon is a massless particle. This is a major source of differences between the electron and neutrino scattering.

In Figure 2.1, $k$ and $k^{\prime}$ are the initial and final electron four-momenta, $p$ and $p^{\prime}$ are the initial and final nucleon four-momenta, and $q^{2}=\left(k-k^{\prime}\right)^{2}=\left(p-p^{\prime}\right)^{2}$ is the four-momentum transfer of the virtual photon. From the QED Feynman rules, the Lorentz invariant matrix element $\mathrm{M}_{f i}$ for the electron-nucleon scattering is,

$$
-i M_{f i}=\left[\bar{v}\left(k^{\prime}\right)\left(i e \gamma^{\mu}\right) u(k)\right]\left(-i \frac{g_{\mu \nu}}{q^{2}}\right)\left[\bar{u}(p)\left(-i e \gamma^{\nu}\right) v\left(p^{\prime}\right)\right] .
$$




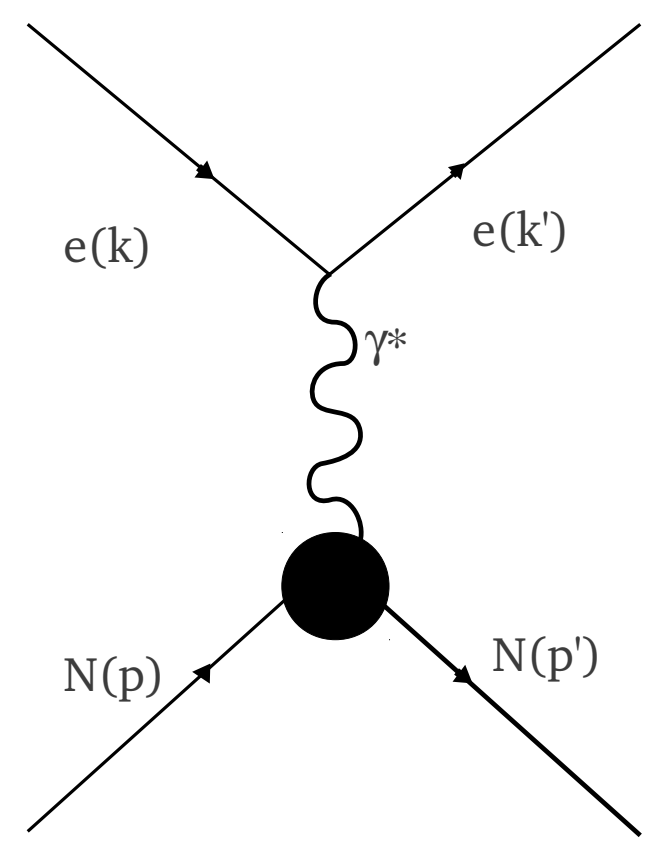

Figure 2.1. The Leading-order (single photon exchange) Quantum Electrodynamics (QED) Feynman diagram.

Here, the nucleon is assumed to be point-like. The currents at the electron-photon vertex, $i e \gamma^{\mu}$ and nucleon-photon vertex, $i e \gamma^{\nu}$, each comes with a factor of the charge (e). The single virtual photon's two couplings yield the strength of the interaction, which is known as the fine-structure constant,

$$
\alpha=\frac{e^{2}}{4 \pi} \approx \frac{1}{137}
$$

Since the fine-structure constant is so small, first-order perturbation theory in $\alpha$ (one photon exchange) is a good approximation for describing the physical process of the electromagnetic interactions [15]. Recall that the coupling strength in the weak interaction is $\alpha_{W} \ll \alpha$, where

$$
\alpha_{W}=\frac{g}{\sqrt{2}}=\frac{\sqrt{4 \pi \alpha}}{\sqrt{2} \sin \theta_{W}}
$$

and the weak mixing angle (Weinberg angle) is measured as $\sin ^{2} \theta_{W}=0.2312$. It is reasonable to conclude that the higher order perturbation corrections may be negligible for the neutrino-nucleon scattering. All the same, the contributions from radiative 
corrections, such as the two boson exchange ( $\mathrm{W} \gamma$ box diagrams), to the neutrino quasielastic cross section has been recently investigated [16]. Based on the theoretical calculations, the $\mathrm{W} \gamma$ contribution is on the order of 2 to 4 percent for the $\nu_{e}$ and $\bar{\nu}_{e}$ and 1 to 2 percent for the $\nu_{\mu}$ and $\bar{\nu}_{\mu}$ cross section [16].

While Equation 2.1.1 describes the invariant amplitude for the elastic scattering off of a point-like Dirac particle, the nucleon is not a structureless particle and is composed of three valence quarks. Beyond the naive quark model, which was discussed earlier, the nucleon is known to be composed of a sea of quark-antiquark pairs and gluons. Thus, the current at the nucleon-photon vertex is modified from a point-like charge distribution to the Fourier transformation of the charge distribution,

$$
F\left(\mathbf{q}^{2}\right)=\int e^{i \mathbf{q} \cdot \mathbf{r}} \rho(\mathbf{r}) d^{3} \mathbf{r}
$$

Here, $\mathrm{F}\left(\mathbf{q}^{2}\right)$ is called the form factor and describes the charge distribution of the nucleon with a finite spatial extent. The nucleon-photon vertex current, $i e \gamma^{\nu}$ is now expressed as $i e \Gamma\left(p^{\prime}, p\right)^{\nu}$. $\Gamma^{\nu}$ is expressed as a linear combination of the bilinear covariants of the Dirac equation [17].

$$
\begin{aligned}
\Gamma\left(p^{\prime}, p\right)^{\nu} & =K_{1} \gamma^{\nu}+i K_{2} \sigma^{\nu \alpha}\left(p^{\prime}-p\right)_{\alpha}+i K_{3} \sigma^{\nu \alpha}\left(p^{\prime}+p\right)_{\alpha} \\
& +K_{4}\left(p^{\prime}-p\right)^{\nu}+K_{5}\left(p^{\prime}+p\right)^{\nu}
\end{aligned}
$$

Here, the structure factors $K_{i}$ are functions of the four-momentum transfer. Parity is known to be conserved in the electromagnetic interactions. So, the terms which include the axial vector components, $\gamma^{5}$, are excluded from the expression in Equation 2.1.3.

As mentioned in Chapter 1, parity is violated in the weak interactions and the neutrino scattering is sensitive to the terms involving the axial vector components. The cross section sensitivity to the axial vector components is another significant difference between the two types of lepton scattering. The axial vector terms of 
the nucleon current will be revisited. Particularly since, the axial vector terms are essential to the neutrino cross section measurement produce in this thesis.

Upon applying the following conditions,

- Lorentz invariance

- Energy and momentum conservation

- Rules of the Dirac gamma matrices

- Vertex current conservation $\left(\partial_{\mu} \mathrm{J}^{\mu}=0\right)$

the expression for $\Gamma^{\nu}$ in Equation 2.1.3 is reduced to

$$
\Gamma^{\nu}=F_{1}\left(q^{2}\right) \gamma^{\nu}+F_{2}\left(q^{2}\right) i \sigma^{\nu \alpha} \frac{q_{\alpha}}{2 M}
$$

Here, $\mathrm{F}_{1}\left(q^{2}\right)$ and $\mathrm{F}_{2}\left(q^{2}\right)$ are called the Dirac and Pauli form factors, respectively. The invariant amplitude in Equation 2.1.1 is rewritten as:

$$
M_{f i}=\frac{e^{2}}{q^{2}} \bar{v}\left(k^{\prime}\right) \gamma^{\mu} u(k) g_{\mu \nu} \bar{u}(p)\left[F_{1}\left(q^{2}\right) \gamma^{\nu}+F_{2}\left(q^{2}\right) i \sigma^{\nu \alpha} \frac{q_{\alpha}}{2 M}\right] v\left(p^{\prime}\right) .
$$

Expressing the Dirac and Pauli form factors as linear combinations,

$$
\begin{gathered}
G_{E}\left(q^{2}\right) \equiv F_{1}\left(q^{2}\right)-\tau F_{2}\left(q^{2}\right) \\
G_{M}\left(q^{2}\right) \equiv F_{1}\left(q^{2}\right)+F_{2}\left(q^{2}\right)
\end{gathered}
$$

provides a way to interpret the physical meaning of the form factors and where

$$
\tau \equiv \frac{Q^{2}}{4 M^{2}}=\frac{-q 2}{4 M^{2}}
$$

$\mathrm{G}_{E}$ and $\mathrm{G}_{M}$ are known as the Sachs electric and magnetic form factors and retain information on the spatial distribution of the electric charge and magnetic moment within the nucleon.

In the low $\mathrm{q}^{2}$ regime, the differential cross section (also known as the Rosenbluth formula) for the electron-nucleon elastic scattering from a finite size target in the lab frame is written as: 


$$
\frac{d \sigma}{d \Omega} \approx \frac{\alpha^{2}}{4 E^{2} \sin ^{2} \theta / 2} \frac{E^{\prime}}{E}\left[G_{E}^{2} \cos ^{2} \theta / 2+\tau G_{M}^{2} \sin ^{2} \theta / 2\right],
$$

where $\mathrm{E}$ and $\mathrm{E}^{\prime}$ are the energies of the incoming and outgoing electron, $\theta$ is the electron scattering angle in the rest frame of the initial nucleon. The Sachs electric and magnetic form factors have been extracted from electron elastic scattering cross sections measurements. The nucleon form factors were extracted using both the Rosenbluth separation (the scattering of an unpolarized electron beam with unpolarized Hydrogen and Deuterium targets) as well as from the polarization transfer measurements. The description of the extraction of the nucleon form factors is beyond the scope of this thesis.

An advantage of the electron-nucleon elastic scattering experiments is that the kinematics can be defined in the Breit frame. In the Breit frame, the energies of the incoming and outgoing electrons are approximately the same. In this picture, the four-momentum transfer, $\mathrm{q}^{2}=\left(\mathrm{k}-\mathrm{k}^{\prime}\right)^{2}$, becomes a purely three-momentum transfer, $\mathrm{q}^{2} \approx\left(0, \mathbf{k}-\mathbf{k}^{\prime}\right)^{2}$. This allows the nucleon form factors to be efficiently extracted using the polarization transfer technique from the electron elastic scattering data. Recall that the nucleon form factors only describe the electric charge and magnetization distributions within the nucleon. The spin structure of the nucleon is characterized by the axial components. The axial component (axial form factor) is measured by the charged pion electro-production and neutrino quasielastic scattering [18]. Experiments have only extracted the parametrization of the axial form factor at low four-momentum transfer, values below $1.0 \mathrm{GeV}^{2}$. The neutrino quasielastic scattering extraction of the axial component is essential for understanding an accurate picture of the nucleons. 


\subsection{NEUTRINO QUASI-ELASTIC SCATTERING}

As mentioned earlier, the neutrino exchanges either a $\mathrm{Z}^{0}$ or $\mathrm{W}^{ \pm}$via the weak nuclear interaction. Neither the neutral current interaction (NC), nor neutrino charged lepton scattering will be discussed within this thesis.

How the neutrino interacts within nuclear matter depends on the neutrino energy [19]. The neutrino can exchange the $\mathrm{W}^{ \pm}$boson with the entire nucleus, the nucleon, or a quark within the nucleon.

\section{- Coherent Scattering}

A property of the neutrino-nucleus coherent scattering is when the neutrino scatters on the entire nucleus with small momentum transfer. In the dynamics of the coherent scattering, vector mesons are produced through the diffractive mechanism [20]. Therefore, the signatures of the CC neutrino and anti-neutrino coherent scattering are $\nu_{l}+A \rightarrow A^{\prime}+l^{-}+\pi^{+}$and $\bar{\nu}_{l}+A \rightarrow A^{\prime}+l^{+}+\pi^{-}$, respectively.

\section{- Quasielastic Scattering}

When the neutrino exchanges a charged gauge boson, $\mathrm{W}^{ \pm}$, with the nucleon the collision is called quasielastic. Here the flavors of the incoming and outgoing leptons differ. For example, in the reaction, $\nu_{e}+\mathrm{n} \rightarrow \mathrm{e}^{-}+\mathrm{p}$, the $\nu_{e}$ changes to an $e^{-}$. In the previous chapter, this process is referred to as the inverse $\beta$-decay.

\section{- Nuclear Resonance Production}

The neutrino can inelastically scattered from a nucleon. The emission of a baryonic excited state, $\Delta$ or $\mathrm{N}^{*}$, is defined as the nuclear resonance production. The baryon decays quickly via the strong interaction. The decay products are usually a nucleon and one or more pions.

\section{- Deep Inelastic Scattering}

The scattering process such that the nucleon breaks apart is known as deep inelastic scattering. The neutrino scatters off of a quark constituent in the nucleon and most likely jet of many hadrons is observed in the hadronic final state. 


\subsubsection{THE NEUTRINO QUASI-ELASTIC DIFFERENTIAL CROSS-SECTION}

The neutrino interaction with the nucleon is the focus of this thesis. For the two-body neutrino-nucleon quasielastic collision,

$$
\begin{gathered}
\nu_{l}+n \rightarrow l^{-}+p \\
\bar{\nu}_{l}+p \rightarrow l^{+}+n,
\end{gathered}
$$

the differential cross section in the nucleon rest frame as function of the four momentum transfer $\left(\mathrm{Q}^{2}=-\mathrm{q}^{2}\right)$ was first derived by C.H. Llewellyn-Smith [21] and is expressed as

$$
\frac{d \sigma}{d Q^{2}}=\frac{M^{2} G_{F}^{2} \cos ^{2} \theta_{C}}{8 \pi E_{\nu}^{2}}\left[A\left(Q^{2}\right) \mp B\left(Q^{2}\right) \frac{(s-u)}{M^{2}}+C\left(Q^{2}\right) \frac{(s-u)^{2}}{M^{4}}\right] .
$$

Here, $\mathrm{M}$ is the mass of the nucleon, $\mathrm{G}_{F}$ is Fermi's constant, $\theta_{C}$ is the Cabibbo angle, $\mathrm{E}_{\nu}$ is the neutrino energy, and $s$ and $u$ are the Mandelstam variables. In the center of mass frame, $s$ and $u$ are defined as,

$$
\begin{aligned}
& s=\left(k^{\mu}+p^{\mu}\right)^{2}=\left(E+M_{n}\right)^{2} \\
& u=\left(k^{\mu}-p^{\mu}\right)^{2}
\end{aligned}
$$

where the initial state nucleon is assumed to be at rest. For the differential cross section (see Equation 2.2.1), the neutrino and anti-neutrino scattering differ by the sign before the $B\left(Q^{2}\right)$ term. The expressions for the functions, $A\left(Q^{2}\right), B\left(Q^{2}\right)$, and $C\left(Q^{2}\right)$ are given by 


$$
\begin{gathered}
A\left(Q^{2}\right)=\frac{\left(m_{l}^{2}+Q^{2}\right)}{M^{2}}\left\{\left[(1+\tau) F_{A}^{2}-(1-\tau)\left(F_{V}^{1}\right)^{2}\right.\right. \\
\left.+\tau(1-\tau)\left(\xi F_{V}^{1}\right)^{2}+4 \tau\left(F_{V}^{1} \xi F_{V}^{2}\right)\right] \\
\left.-\frac{m_{l}^{2}}{4 M^{2}}\left[\left(F_{V}^{1}+\xi F_{V}^{2}\right)^{2}+\left(F_{A}+2 F_{P}\right)^{2}-4(1+\tau) F_{P}^{2}\right]\right\} \\
B\left(Q^{2}\right)=\frac{Q^{2}}{M^{2}} F_{A}\left(F_{V}^{1}+\xi F_{V}^{2}\right) \\
C\left(Q^{2}\right)=\frac{1}{4}\left(F_{A}^{2}+\left(F_{V}^{1}\right)^{2}+\tau\left(\xi F_{V}^{2}\right)^{2}\right) .
\end{gathered}
$$

Here, $\xi=\left(\mu_{p}-1\right)-\mu_{n}$ and $\mu_{p}$ and $\mu_{n}$ are the proton and neutron magnetic moments and $\tau=\frac{Q^{2}}{4 M^{2}}$.

The scalar, $\mathrm{F}_{V}^{3}$, and tensor, $\mathrm{F}_{A}^{3}$, form factors are excluded from the above expressions. The $\mathrm{F}_{V}^{3}$ and $\mathrm{F}_{A}^{3}$ are responsible for the $\mathrm{G}$-parity violating second-class currents. The G-parity transformation is a discrete symmetry that is a compound operation on the charge conjugation and isospin rotation of the pion field about the isospin projected axis [22]. The G-parity operator is written as

$$
G=C e^{i \pi I_{2}}
$$

where $C$ is the charge conjugation and $I$ is the isospin projection along an axis. The G-parity transformation is used to study particle decays into pions. The bubble chamber neutrino and anti-neutrino scattering experiments did not find any evidence for possible effects from the second-class currents to the neutrino and anti-neutrino quasielastic cross sections [23]. Due to the lack of proof, the second-class currents are usually assumed to be negligible. Under this assumption, the form factors, $\mathrm{F}_{V}^{3}$ and $\mathrm{F}_{A}^{3}$, are set to equal zero and do not appear in the functions (see Equations 2.2.3, 2.2.4, and 2.2.5). 
The form factors are assumed to be real. This implies that the time-reversal symmetry is invariant in the neutrino-nucleon quasi-elastic scattering [24]. After applying the conditions just discussed, the functions, $A\left(Q^{2}\right), B\left(Q^{2}\right)$, and $C\left(Q^{2}\right)$ are expressed in terms of the vector form factors, $\mathrm{F}_{V}^{1}$ and $\mathrm{F}_{V}^{2}$, the axial form factor $\mathrm{F}_{A}$, and the pseudoscalar form factor $\mathrm{F}_{P}$. The expressions are illustrated in Equations $2.2 .3,2.2 .4$, and 2.2.5.

\subsubsection{THE VECTOR FORM FACTORS}

The vector form factors can be related to the nucleon electromagnetic form factors measured in electron elastic scattering by the conservation of vector current (CVC), and can be written as,

$$
\begin{aligned}
F_{V}^{1}\left(Q^{2}\right) & =\frac{G_{E}^{V}\left(Q^{2}\right)-\tau G_{M}^{V}\left(Q^{2}\right)}{1-\tau} \\
\xi F_{V}^{2}\left(Q^{2}\right) & =\frac{G_{M}^{V}\left(Q^{2}\right)-G_{E}^{V}\left(Q^{2}\right)}{1-\tau}
\end{aligned}
$$

$\mathrm{G}_{E}^{V}$ and $\mathrm{G}_{M}^{V}$ are expressed as a linear combination of the proton and neutron electric and magnetic form factors,

$$
\begin{aligned}
& G_{E}^{V}\left(Q^{2}\right)=G_{E}^{p}\left(Q^{2}\right)-G_{E}^{n}\left(Q^{2}\right) \\
& G_{M}^{V}\left(Q^{2}\right)=G_{M}^{p}\left(Q^{2}\right)-G_{M}^{n}\left(Q^{2}\right) .
\end{aligned}
$$

The simplest parametrization of the Sachs form factors is the dipole approximation $[25]$.

$$
\begin{aligned}
& G_{E}^{p}\left(Q^{2}\right)=G_{D}\left(Q^{2}\right), \\
& G_{E}^{n}\left(Q^{2}\right)=0, \\
& G_{M}^{p}\left(Q^{2}\right)=\mu_{p} G_{D}\left(Q^{2}\right), \\
& G_{M}^{n}\left(Q^{2}\right)=\mu_{n} G_{D}\left(Q^{2}\right),
\end{aligned}
$$


where the dipole form is given by,

$$
G_{D}\left(Q^{2}\right)=\frac{1}{\left(1+\frac{Q^{2}}{M_{V}^{2}}\right)^{2}}
$$

and $\mathrm{M}_{V}$ is the vector mass parameter with an extracted value of $0.71 \mathrm{GeV}^{2}$. From the electron data, the dipole form describes the $\mathrm{Q}^{2}$-dependence of the electric and magnetic form factors of the proton very well for low values of $\mathrm{Q}^{2}$, for values below $2.0 \mathrm{GeV}^{2}$ [17]. The magnetic form factor of the neutron is also adequately modeled by the dipole form in the low $\mathrm{Q}^{2}$ regime. As mentioned in Section 2.1, the nucleons form factor are extracted using two different techniques, the Rosenbluth separation and polarization transfer. Only under the polarization transfer method does the dipole approximation fail to describe the extracted values of $\mu_{p} G_{E}^{p} / G_{M}^{p}$ for values of $\mathrm{Q}^{2}$ above $2.0 \mathrm{GeV}^{2}$. It is generally stated that the polarization transfer method is sensitive to the two-photon exchange ( $\gamma \gamma$ box diagrams) process.

Due to the discrepancy that exist between the Rosenbluth separation and the polarization transfer techniques, many neutrino experiments are using the nucleon form factor extraction that takes into account a fit to the electron elastic scattering data, instead of the dipole approximation. One such fit is due to Bodek, Budd, Bradford, and Arrington and is called the BBBA07 Form Factors [26], [27]. The BBBA07 Form Factors are discussed in more detail in a later chapter.

\subsubsection{THE NUCLEAR $\beta$-DECAY HADRONIC CURRENT}

The general expression of the hadronic current for the nuclear $\beta$-decay is written as $[22]$,

$$
\left\langle p\left|A_{\mu}^{\dagger}\right| n\right\rangle=\bar{p}\left[\gamma_{\mu} F_{A} \gamma_{5}+\frac{q_{\mu}}{M} F_{P} \gamma_{5}\right] n
$$


If the pseudoscalar term is ignored and $F_{A}\left(Q^{2} \rightarrow 0\right)=g_{W}$, the Equation 2.2 .11 describes the point-like $\beta$-decay discussed in Chapter 1. As written, the Equation 2.2.11 does not account for terms such as the pion vertex corrections, where an example is shown in Figure 2.2. These types of terms should be added in order to describe the total contribution of the hadronic current to the cross section. In fact, the pion vertex correction terms contribute as a higher order correction to the axial vector component and are difficult to calculate [22]. However, there does exist the one-pion exchange correction, where a pion is exchange between the nucleons as illustrated in Figure 2.3

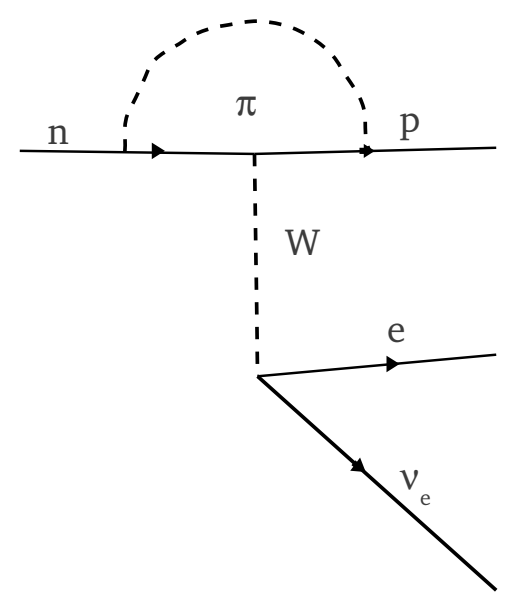

Figure 2.2. The pion vertex correction diagram.

The pseudoscalar interaction provides a descriptive picture of the one-pion exchange and serves as the primary contribution to the pseudoscalar form factor, $F_{P}\left(Q^{2}\right)$ [22]. The amplitude of the one-pion exchange of the hadronic current (see Figure 2.3) corresponds to three distinct components, pion emission by the neutron, pion propagation, and pion decay, 


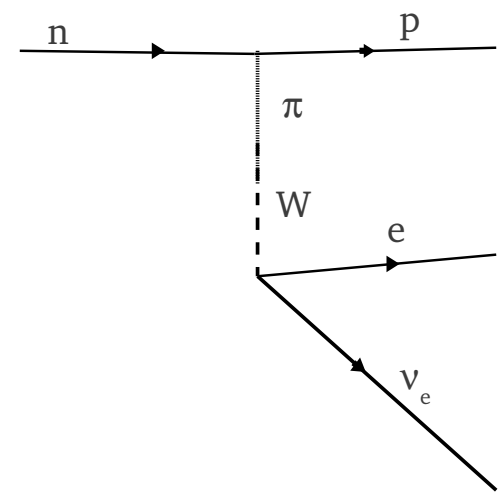

Figure 2.3. The one-pion exchanged diagram.

$$
\begin{aligned}
& A \sim[n \rightarrow p+\pi] \times[\pi-\text { propagator }] \times[\pi \rightarrow l+\nu] \\
& A \sim\left[i g_{0}\left(\bar{p} \gamma_{5} n\right)\right] \times\left[\frac{1}{q^{2}-m_{\pi}^{2}}\right] \times\left[\frac{G_{F} \cos \theta_{C}}{\sqrt{2}} i f_{\pi} q_{\mu}\left(\bar{l} \gamma^{\mu}\left(1-\gamma_{5}\right) \nu\right)\right],
\end{aligned}
$$

where $g_{0}$ and $f_{\pi}$ are the pion-nucleon coupling constant and pion decay constant. The pseudoscalar form factor is characterized by the pion pole contribution, so the hadronic current for the nuclear $\beta$-decay in Equation 2.2.11 is rewritten as,

$$
\left\langle p\left|A_{\mu}^{\dagger}\right| n\right\rangle=\bar{p}\left[\gamma_{\mu} F_{A}-\frac{q_{\mu}}{M} \frac{g_{0} f_{\pi}}{q^{2}-m_{\pi}^{2}}\right] \gamma_{5} n
$$

\subsubsection{THE PSEUDOSCALAR FORM FACTOR}

The axial current is not a conserved theory, but under the theorem of partial conservation of axial current (PCAC), it is conserved in the limit when $m_{\pi} \rightarrow 0$. So that, 


$$
\begin{aligned}
0 & =\lim _{m_{\pi} \rightarrow 0} \partial^{\mu} A_{\mu} \\
& =\lim _{m_{\pi} \rightarrow 0} \bar{p} \partial^{\mu}\left(\gamma_{\mu} F_{A}-\frac{q_{\mu}}{M} \frac{g_{0} f_{\pi}}{q^{2}-m_{\pi}^{2}}\right) \gamma_{5} n \\
& =\lim _{m_{\pi} \rightarrow 0} \bar{p}\left[\gamma_{\mu} \partial^{\mu} F_{A}-\frac{q^{2}}{M} \frac{g_{0} f_{\pi}}{q^{2}-m_{\pi}^{2}}\right] \gamma_{5} n \\
& =\lim _{m_{\pi} \rightarrow 0} \bar{p}\left[\not \partial F_{A}-\frac{q^{2}}{M} \frac{g_{0} f_{\pi}}{q^{2}-m_{\pi}^{2}}\right] \gamma_{5} n \\
& =\lim _{m_{\pi} \rightarrow 0} \bar{p}\left[\left(\not p_{p}-\not p_{n}\right) F_{A}-\frac{q^{2}}{M} \frac{g_{0} f_{\pi}}{q^{2}-m_{\pi}^{2}}\right] \gamma_{5} n \\
& =\lim _{m_{\pi} \rightarrow 0} \bar{p}\left[\left(m_{p}+m_{n}\right) F_{A}-\frac{q^{2}}{M} \frac{g_{0} f_{\pi}}{q^{2}-m_{\pi}^{2}}\right] \gamma_{5} n \\
& =\lim _{m_{\pi} \rightarrow 0} \bar{p}\left[2 M F_{A}-\frac{q^{2}}{M} \frac{g_{0} f_{\pi}}{q^{2}-m_{\pi}^{2}}\right] \gamma_{5} n \\
& =\bar{p}\left[2 M F_{A}-\frac{g_{0} f_{\pi}}{M}\right] \gamma_{5} n .
\end{aligned}
$$

This implies that,

$$
g_{0} f_{\pi}=2 M^{2} F_{A}
$$

where the result is known as the Goldberger-Treiman relation [22]. Due to the onepion exchange vertex correction, the pseudoscalar form factor can be related to the axial form factor,

$$
F_{P}=-\frac{g_{0} f_{\pi}}{q^{2}-m_{\pi}^{2}}=\frac{2 M^{2}}{Q^{2}+m_{\pi}^{2}} F_{A} .
$$

In the cross section formula, (see Equation 2.2.1) notice that $\mathrm{F}_{P}\left(Q^{2}\right)$ is multiplied by $\frac{m_{l}}{M}$, where $m_{l}$ and $M$ are the masses of the outgoing lepton and nucleon, respectively. As a result, the pseudoscalar form factor makes a small contribution to the cross section for the $e N$ elastic and $\nu_{\mu} \mathrm{N}$ quasi-elastic scattering. 


\subsubsection{THE AXIAL FORM FACTOR}

The extracted Sachs form factors from the electron elastic scattering data (at low values of $\mathrm{Q}^{2}$ ) supports the dipole approximation for describing the electric charge and magnetic moment distributions within the nucleons. For that reason, the axial form factor is modeled by the dipole form,

$$
F_{A}\left(Q^{2}\right)=\frac{g_{A}}{\left(1+\frac{Q^{2}}{M_{A}^{2}}\right)^{2}} .
$$

Here, $\mathrm{M}_{A}$ is the axial mass parameter and $\mathrm{g}_{A}$ is the axial coupling constant. In the limit of $\mathrm{Q}^{2} \rightarrow 0, \mathrm{~F}_{A}\left(Q^{2}=0\right)$ is essentially the axial coupling constant and has been measured by the nuclear $\beta$-decay experiments. It is also equivalent to the difference between the spin contributions from the up and down quarks in the nucleon [28],

$$
g_{A}=\Delta u-\Delta d=-1.267
$$

Argonne (1969)

Argonne (1973)

CERN (1977)

Argonne (1977)

CERN (1979)

BNL (1980)

BNL (1981)

Argonne (1982)

Fermilab (1983)

BNL (1986)

BNL (1987)

BNL (1990)

Average

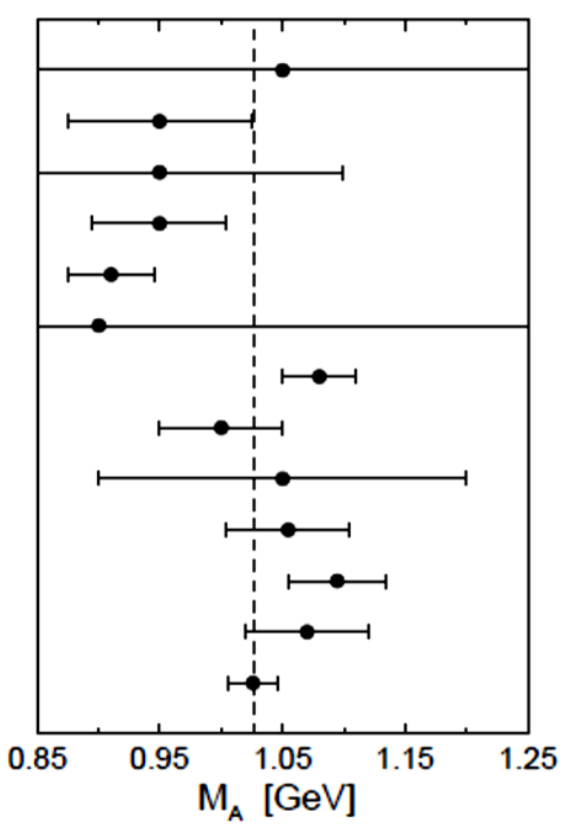

Figure 2.4. The axial mass extraction from neutrino and anti-neutrino quasielastic scattering experiments. Reprinted from Reference [29], Copyright (2001). 
As mentioned earlier, $\mathrm{M}_{A}$ is the free parameter that is extracted from neutrino and anti-neutrino quasielastic scattering experiments. The original set of $\mathrm{M}_{A}$ measurements was reported from neutrino scattering experiments on deuterium [29]. In Figure 2.4, the extracted values of $\mathrm{M}_{A}$ from both the neutrino and anti-neutrino experiments are displayed, where the world average value is

$$
M_{A}=1.026 \pm 0.021 \mathrm{GeV}
$$

For these neutrino experiments, there are large uncertainties on the neutrino flux and the theoretical predictions of the neutrino interactions [29]. These uncertainties are propagated to the extracted values of $\mathrm{M}_{A}$. The Table 2.2.5 shows the event rate for neutrino experiments on deuterium [30], at $\mathrm{Q}^{2}>1.0 \mathrm{GeV}^{2}$, where the statistical errors are large.

Recall that the nucleon electric and magnetic form factors are $\mathrm{Q}^{2}$ dependent. As $\mathrm{Q}^{2}$ becomes larger, the magnetic contributions to the cross section begin to dominate, as observed in the electron elastic scattering data. Since electron elastic scattering experiments are able to make measurements across a wide range of $\mathrm{Q}^{2}$, the contributions of the electric charge and magnetization distributions of the nucleon can be fully explored. Thus far in neutrino quasielastic scattering experiments, the cross section is measured for limit range of $\mathrm{Q}^{2}$ (see Table 2.2.5) [30].

Table 2.1. The Published $\mathrm{M}_{A}$ values by $\nu$-deuterium Experiments. Reprinted from Reference [30], Copyright (2007).

\begin{tabular}{|l|l|l|l|}
\hline Experiment & QE events & $\mathrm{Q}^{2}$ range $\left(\mathrm{GeV} / \mathrm{c}^{2}\right)$ & $\mathrm{M}_{A}$ \\
\hline Mann $_{73}$ & 166 & $0.05-1.6$ & $0.95 \pm .12$ \\
\hline Barish $_{77}$ & 500 & $0.05-1.6$ & $0.95 \pm .09$ \\
\hline Miller $_{82,77,73}$ & 1737 & $0.05-2.5$ & $1.00 \pm .05$ \\
\hline Baker $_{81}$ & 1138 & $0.06-3.0$ & $1.07 \pm .06$ \\
\hline Kitagaki $_{83}$ & 362 & $0.11-3.0$ & $1.05 \pm_{-.16}^{+.12}$ \\
\hline Kitagaki $_{90}$ & 2544 & $0.10-3.0$ & $1.070 \pm_{-.045}^{+.040}$ \\
\hline Allasia $_{90}$ & 552 & $0.1-3.75$ & $1.080 \pm .08$ \\
\hline
\end{tabular}


As result, the dipole approximation of the axial form factor has not been successfully validated for higher values of $\mathrm{Q}^{2}$. Recall that the axial structure provides information on the nucleon's spin. In the limit of $\mathrm{Q}^{2} \rightarrow 0$, the total spin contribution of the nucleon comes from the valence quarks,

$$
\mathrm{F}_{A}\left(Q^{2}=0\right)=\mathrm{g}_{A}=\Delta \mathrm{u}-\Delta \mathrm{d}=-1.267,
$$

and the spatial distribution of the quarks becomes significant at higher values of $\mathrm{Q}^{2}$. Beyond the simple picture of the quark model, the nucleon is composed of valence and sea quarks, as well as gluons that fluctuate within the strong color field of the valence quarks [17]. Because the nucleon is a complex system, it is important for the current generation of neutrino scattering experiments to produce measurements across a wide range of $\mathrm{Q}^{2}$, so that the axial structure of the nucleon can be fully examined. Using the neutrino to probe the nucleon has not been investigated as extensively as the electron scattering, because neutrinos interact weakly.

\subsection{SCATTERING FROM HEAVIER NUCLEI}

For higher event rates, the modern neutrino scattering experiments are composed of nuclei with $\mathrm{A}>2$. For example, the NOMAD and MiniBooNE experiments are assembled primarily from carbon-based materials [31], [32]. As a result, the neutrino scatters from a bound nucleon inside the nucleus. This is unlike the first generation of neutrino experiments where the neutrino or anti-neutrino scatters from a Deuterium or Hydrogen target. In general, the $\nu$-Deuterium or $\bar{\nu}$-Hydrogen scattering is treated as QE scattering from a free neutron or a free proton.

The neutrino event generators for the modern neutrino experiments use the Relativistic Fermi Gas model (RFG) and the plane-wave impulse approximation (PWIA) to simulate the QE scattering from the nucleus [33]. The RFG model describes the nucleus as a system of quasi-free nucleons bound by a nuclear potential and does not 
account for the nuclear shell structure. However, the Fermi motion of the nucleons inside of the nucleus and the Pauli blocking effect are taken into account [34], where the Fermi motion is only done approximately. The Pauli blocking implementation requires that the recoil nucleon is outside of the Fermi sea. Using this simple picture of the nucleus, the NOMAD and MiniBooNE experiments measured the total QE cross section for the neutrino scattering as function of the neutrino energy and the results are displayed in Figure 2.5. Also, Figure 2.6 shows the most recent extracted values for $\mathrm{M}_{A}$ from the neutrino experiments on the carbon nucleus as well as oxygen and iron nuclei [35]. Note that $\mathrm{M}_{A}$ is extracted for different regions of $\mathrm{Q}^{2}$ for each experiment.

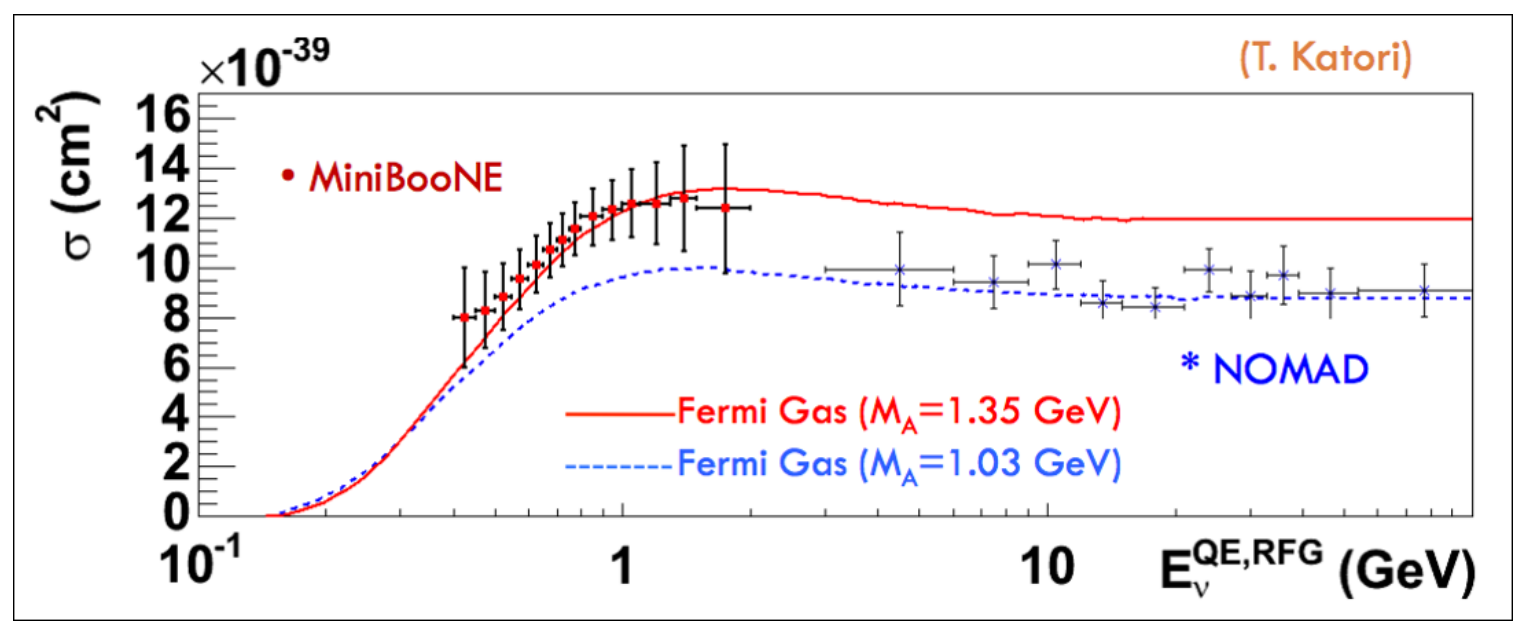

Figure 2.5. The total QE cross section for the NOMAD and MiniBooNE experiments. Reprinted from [36], Copyright (2010) with permissions from MiniBooNE Collaboration.

Figures 2.5 and 2.6 show that there exist discrepancies in the extracted values of $\mathrm{M}_{A}$ between the modern neutrino experiments, which is referred to as the axial mass anomaly. The carbon-based neutrino detectors, NOMAD and MiniBooNE were the first to observe the discrepancy as indicated in Figure 2.5. The extracted $\mathrm{M}_{A}$ values displayed in Figure 2.4 confirms that the NOMAD data is consistent with neutrino scattering on a free neutron target. However, the MiniBooNE data prefers a significantly higher value of $\mathrm{M}_{A}=1.35 \pm 0.17 \mathrm{GeV}[36]$. 


\begin{tabular}{|c|c|c|c|}
\hline Experiment & Target & Cut in $Q^{2}\left[\mathrm{GeV}^{2}\right]$ & $M_{A}[\mathrm{GeV}]$ \\
\hline $\mathrm{K} 2 \mathrm{~K}$ & oxygen & $Q^{2}>0.2$ & $1.2 \pm 0.12$ \\
$\mathrm{~K} 2 \mathrm{~K}$ & carbon & $Q^{2}>0.2$ & $1.14 \pm 0.11$ \\
MINOS & iron & no cut & $1.19 \pm 0.17$ \\
MINOS & iron & $Q^{2}>0.2$ & $1.26 \pm 0.17$ \\
MiniBooNE & carbon & no cut & $1.35 \pm 0.17$ \\
MiniBooNE & carbon & $Q^{2}>0.25$ & $1.27 \pm 0.14$ \\
\hline \hline NOMAD & carbon & no cut & $1.07 \pm 0.07$ \\
\hline
\end{tabular}

Figure 2.6. The most recent $\mathrm{M}_{A}$ measurements on Heavy Nuclei. Reprinted from Reference [35], Copyright (2010).

Notice that the NOMAD experiment measures the cross section at higher values of the neutrino energy $\left(\mathrm{E}_{\nu}>3 \mathrm{GeV}\right)$, while MiniBooNE measures the cross section for lower values of the neutrino energy $\left(\mathrm{E}_{\nu}<3 \mathrm{GeV}\right)$. This also means that the NOMAD and MiniBooNE experiments measured the QE cross section in different regions of $\mathrm{Q}^{2}$. Equations 2.3.1 show the relationship between the $\mathrm{Q}^{2}$ and $\mathrm{E}_{\nu}$, under the $\mathrm{QE}$ hypothesis and using the lepton's kinematics.

$$
\begin{aligned}
E_{\nu} & =\frac{2\left(M_{n}-E_{B}\right) E_{l}-\left[\left(M_{n}-E_{B}\right)^{2}+m_{l}^{2}-M_{p}^{2}\right]}{2\left[M_{n}-E_{B}-E_{l}-p_{l} \cos \theta_{l}\right]} \\
Q^{2} & =-m_{l}^{2}+2 E_{\nu}\left(E_{l}-\sqrt{E_{l}^{2}-m_{l}^{2}} \cos \theta_{l}\right)
\end{aligned}
$$

The $\sim 30 \%$ discrepancy in the data between the NOMAD and MiniBooNE experiments is $\mathrm{Q}^{2}$ dependent. The lack of sophisticate modeling of the nuclear effects in the neutrino event generators is one way to interpret the differences that are observed. In fact, the electron scattering data gives rise to the conclusion that the RFG model does not completely account for the nuclear dynamics in the low $\mathrm{Q}^{2}$ regime 
[37]. Hence, the MiniBooNE data requires an effective change in the axial form factor mass parameter, $\mathrm{M}_{A}$, to compensate for the unaccounted for nuclear effects.

As mentioned previously, the PWIA approximation suggests that the incoming neutrino exchanges a gauge boson with a single nucleon in the nucleus. The electronnucleus scattering experiments have quantified that PWIA approximation breaks down at $\mathrm{Q}^{2}<400(\mathrm{MeV} / \mathrm{c})^{2}$ [38]. The de Broglie wavelength of the gauge boson, $\lambda=\frac{\hbar}{\left|\mathbf{q}^{2}\right|} \approx \frac{0.2 \mathrm{fm}}{Q^{2}}$, corresponds to how well a single nucleon is viewed. At low values of $\mathrm{Q}^{2}$, the neutrino probes a region that is covered by multiple nucleons. Therefore, more than one particle is involved in the scattering process. As a result, QE scattering on nucleons in nuclei is sensitive to nuclear effects such as nucleon-nucleon (NN) interactions and two-body currents.

\subsubsection{NUCLEON-NUCLEON SHORT RANGE CORRELA- TIONS}

The strong nucleon-nucleon force is responsible for the structure of the nuclei and attractive at intermediate ranges and repulsive at short distances. The nucleons in the nucleus can be thought of as waves overlapping for short periods of time, which is referred to as nucleon-nucleon short range correlations (SRC) [39]. Figure 2.7 shows examples of nucleon-nucleon correlations [40].

The nucleon-nucleon SRC is often explained as excitations in the nucleus where two nucleons that are very close together undergo a hard interaction due to the strong nuclear force. The nucleons are left in a configuration such that each nucleon has a large momentum (the nucleon momentum is greater than the Fermi momentum) but a small total momentum [42]. This means that the momentum vectors of the nucleons are aligned back-to-back relative to each other. When probing these configurations in the inclusive electron scattering, the correlated pair effectively breaks apart and the scattered nucleon's partner is knocked-out of the nucleus in the process [39]. Recent 


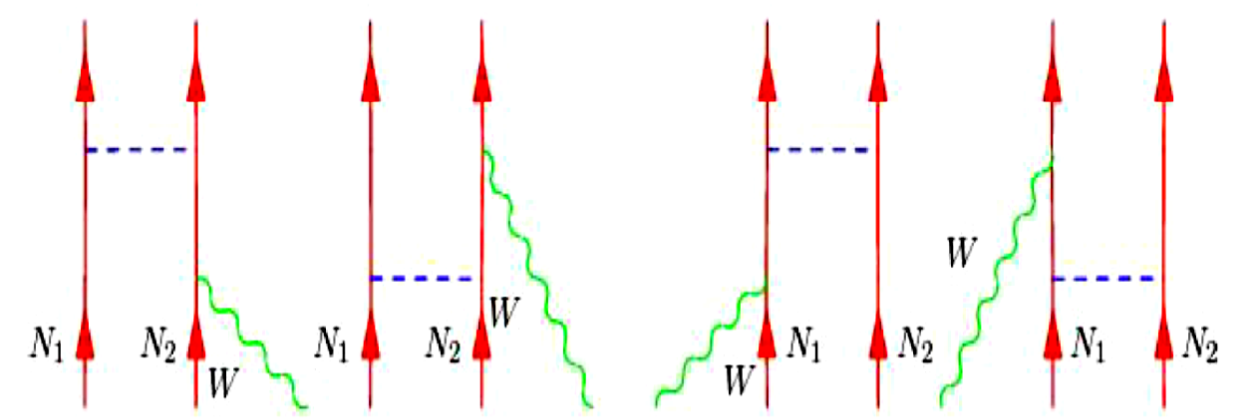

Figure 2.7. The Feynman diagrams of nucleon-nucleon correlations in the nuclear ground state. The wiggle lines, dotted lines, solid lines correspond to the virtual boson, pion, and the nucleons, respectively. The boson is absorbed by a correlated nucleon pair, where a pion is exchanged between the nucleons. Reprinted from Reference [41] with permissions from J. Sobczyk.

measurements from the inclusive electron scattering experiments have suggested that about $20 \%$ of the nucleons in the carbon nucleus are in this state at any given time [39].

\subsubsection{Meson Exchange Currents}

Another mechanism that is omitted in the description of nuclear dynamics is the two-body current phenomenon. The two-body currents are not the same as short range nucleon-nucleon correlations. The meson exchange currents (MEC) are twobody currents that are carried by a virtual meson that is exchanged between two nucleons in the nucleus [37]. Therefore, MEC can excite both the one-particle one-hole (1p-1h) and two-particle two-hole (2p-2h) states [37]. Like the nucleon-nucleon SRC

process, the $2 \mathrm{p}-2 \mathrm{~h}$ MEC leads to the emission of two nucleons in the hadronic final state. Figure 2.8 shows examples of the Feynman diagrams for the meson exchange currents. Although the dynamical aspects of the MEC differ from the absorption 
of the virtual boson by a correlated nucleon pair, the MEC and SRC cannot be distinguished by kinematics. For that reason, theoretical models, that illustrate each component contribution to the total cross section are needed to compare and validate with the measured data.
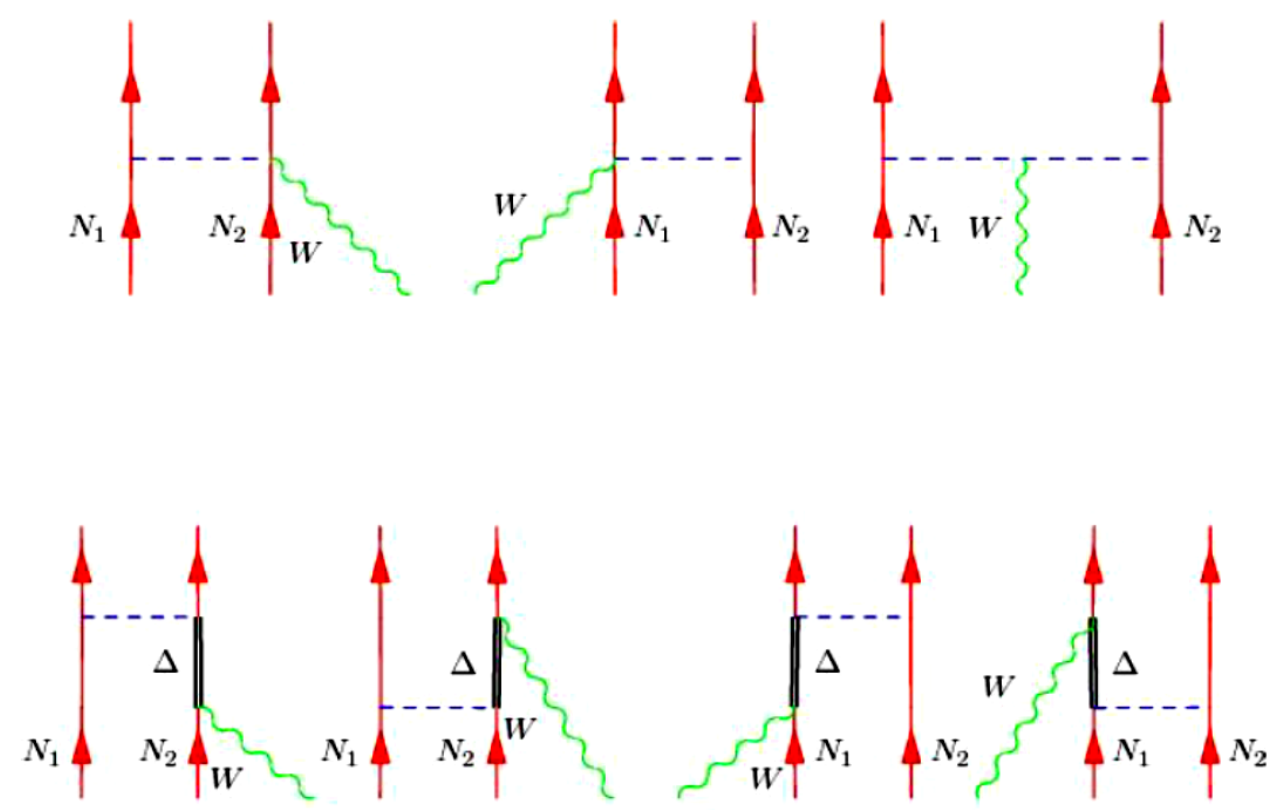

Figure 2.8. Example Feynman diagrams of the meson exchange currents. The top Figures are known as the contact and pion-in-flight diagrams and the bottom Figures are called $\Delta$-MEC diagram. Reprinted from Reference [41] with permissions from J. Sobczyk.

There are several theoretical computations that include the additions to the CC cross section due to the multinucleon contributions for the neutrino scattering from heavier nuclei [43]. The MEChM model (developed by Martini, Ericson, Chanfray, and Marteau) predicts a significant increase in the CC cross section due to the nparticles n-holes (np-nh) states for explaining the MiniBooNE anomalous $\mathrm{M}_{A}$ measurement [44]. However, there are some limits to the current microphysical models. For example, the calculations are based on effective theories that are only valid in a specific kinematic space [45]. 
Apart from the theoretical computations, there exists the Transverse Enhancement Model (TEM), which is an empirical approach that accounts for the increase in the transverse part of the QE cross section due to the multinucleon affects [46]. The TEM model is based on the inclusive electron scattering data by fitting the additional strength to the cross section which is beyond the independent nucleon impulse approximation.

\subsubsection{THE EFFECTS ON RECONSTRUCTION}

For the neutrino scattering experiments, the energy of the incoming neutrino beam is unknown. The neutrino energy must be reconstructed from kinematics. Consequently, there are implications in the neutrino energy reconstruction for excluding multinucleons from the picture of the nucleus. Figure 2.9 shows a theoretical computation of the reconstructed neutrino energy for three different $\mathrm{E}_{\nu}$ values, where the dashed lines are the pure quasielastic events and the solid lines are the quasielastic events with the multinucleon contributions [47]. The neutrino energy is reconstructed using only the muon kinematics. In Figure 2.9, $\overline{\mathrm{E}}_{\nu}$ is the reconstructed value of $\mathrm{E}_{\nu}$.

For the QE scattering, the neutrino energy is reconstructed assuming the scattering off of a single nucleon bound in the nucleus. Figure 2.9 illustrates that the low-side tail of the energy distributions is dominated by the n-particles n-holes phenomenon. Therefore, applying Equation 2.3.1 to reconstruct the neutrino energy is incorrect. In addition, if the neutrino event generator does not simulate the hadronic side of the interaction properly, it makes the detector calorimetric reconstruction more challenging [48]. Because the calorimetric reconstruction depends on the number of hits observed in the detector, where the hits originate from all of the generated particles in the final state. For the neutrino-nucleus scattering, it is critical to accurately describe the dynamics of the nuclear system. Some possible consequences for excluding the additional nuclear effects are unexplained discrepancies between the neutrino and 


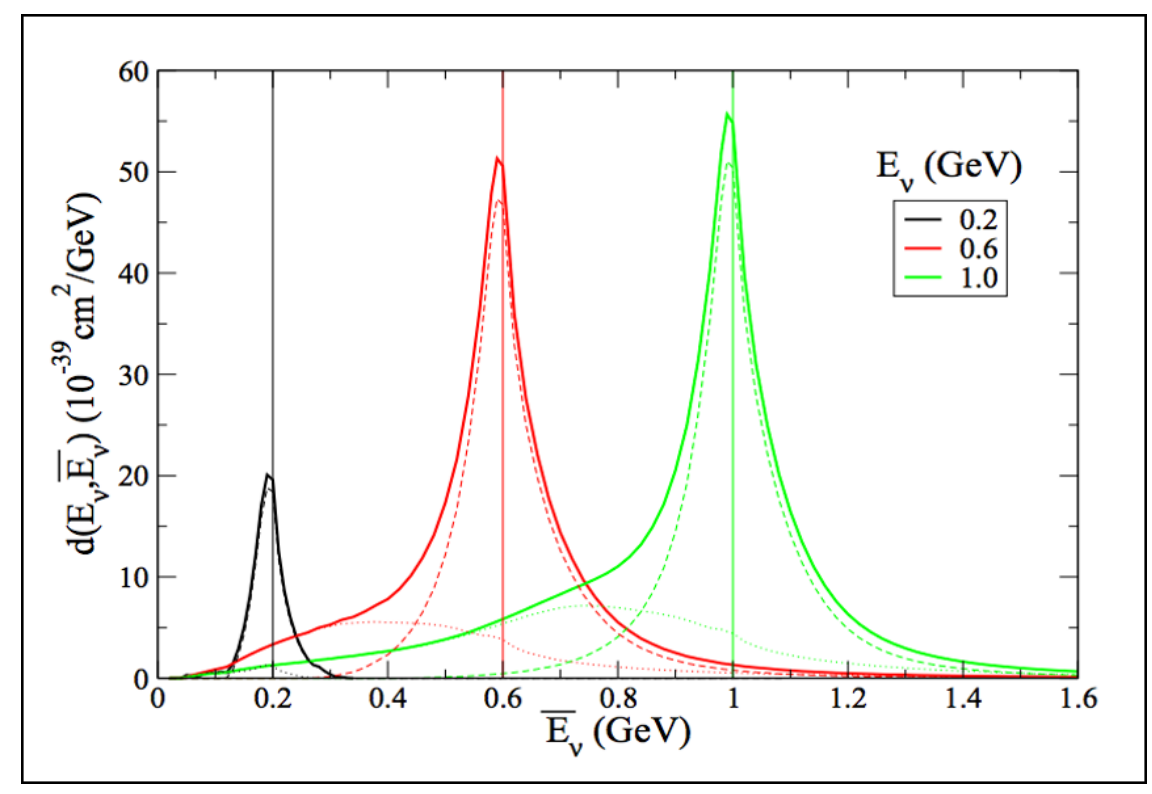

Figure 2.9. The theoretical computation of the neutrino energy spread for three $\mathrm{E}_{\nu}$ values on the Carbon nucleus, where $\overline{\mathrm{E}}_{\nu}$ is the smeared value of the generated neutrino energy, $\mathrm{E}_{\nu}$. Reprinted from Reference [47], Copyright (2012).

electron scattering data, large uncertainties on the cross section measurements, and unreliable outcomes from the kinematic reconstruction.

\subsection{THE EFFECTS OF THE FINAL STATE INTERAC- TIONS}

Thus far, only the nuclear physics at the scattering vertex has been discussed. The other important ingredient of the QE scattering from heavy nuclei is the modeling of the final state interactions (FSI) between the ejected nucleon and the residual nucleus. There are a couple of key factors of how FSI influence the cross section measurement. Foremost, a major problem for studying the nucleon-nucleon SRC in the electron scattering experiments is the FSI effects that occur between the particles in the final state [42]. Because nucleon propagation through the nucleus plays a critical role in the understanding of the kinematical correlations between the struck and spectator nucleons [42]. The FSI is independent of the probe use to study the 
structure of the nucleon [49]. Under the influence of FSI, those nucleons can interact with the residual nucleus before escaping. If the sensitivity to nuclear effects differ in the electron and neutrino scattering, then how the FSI effects obscure nuclear effects in electron scattering when compared to neutrino scattering may also diverge.

The FSI plays a significant part in the identification of the quasielastic events for the modern neutrino experiments. Ignoring the nuclear effects, the signature of a genuine quasielastic event is one lepton and one nucleon in the final state. The struck nucleon can be absorbed by the residual nucleus or inelastically re-scattered before escaping the nucleus. After the hadron propagation through the nucleus, the signature of the quasielastic event may no longer be observed as a two-body system. In some scenarios, the non-quasielastic events, such as the resonance production and deep inelastic scattering (DIS), can mimic the quasielastic signature. For example, the most common reaction of this type is the process, $\nu_{l}+p \rightarrow \Delta^{++}+l^{-} \rightarrow l^{-}+$ $p+\pi^{+}$. If the pion is absorbed, the $\Delta^{++}$production is observed as a quasielasticlike event. For studying the effects of FSI, it is essential to define a signal based on the expected signature of the neutrino interaction, instead of the neutrino event generator definition of the signal. By defining the signal based on the event generator definition, the analysis becomes very sensitive to the modeling of FSI, where neutrino event generators rely on intra-nuclear cascade (INC) models to describe the hadrons propagation in the nucleus [50]. The INC model is discussed in more detail in a later chapter. Understanding the implications of the FSI effects in the neutrino event generators the central theme of this dissertation. 


\section{SECTION 3}

\section{THE MINER $\nu$ A EXPERIMENT}

MINER $\nu \mathrm{A}$ (Main INjector ExpeRiment $\nu$-A) is a neutrino scattering experiment located at Fermi National Accelerator Laboratory (FNAL, Fermilab) [51]. The MINER $\nu \mathrm{A}$ detector is composed of fine-grained polystyrene scintillator for high quality tracking. The upstream region of the detector consists of various solid and liquid nuclear targets which are utilized for studying the A-dependence of neutrino interactions. In addition to the tracking scintillator core, the detector employs regions for electromagnetic and hadronic calorimetry. The NuMI (Neutrinos at the Main Injector) beamline exposes MINER $\nu \mathrm{A}$ to a high intensity flux of neutrino or antineutrino particles which give rise to the neutrino collisions observed in the detector. The MINOS (Main Injector Neutrino Oscillation Search) near detector lives downstream of MINER $\nu \mathrm{A}$ and serves as a muon spectrometer. This chapter describes the various apparatus components of the MINER $\nu \mathrm{A}$ experiment that are needed to produce neutrino cross section measurements.

\subsection{THE NuMI BEAMLINE}

NuMI, which is part of the Fermilab Accelerator Complex, is a two-horn focused, accelerator neutrino beam that was designed initially for short and long-baseline, neutrino oscillation experiments [52]. Figure 3.1 shows a schematic diagram of the NuMI beamline. With respect to the Earth's surface, the NuMI beamline is directed into the Earth at an angle of $58 \mathrm{mrad}$ so that the center of the neutrino beam propagates through the MINOS far detector which is located in Soudan, MN [53]. The Fermilab Main Injector delivers a primary beam of $120 \mathrm{GeV} / \mathrm{c}$ protons to the NuMI graphite (Carbon) production target [54]. A spray of mesons consisting primarily of pions 
and kaons are produced from the proton-Carbon $(p C)$ collisions. Along the direction of the beam axis, the magnetic horns focus or de-focus the charged mesons which subsequently decay in flight to produce a tertiary beam that is predominately $\nu_{\mu}$ or $\bar{\nu}_{\mu}$ particles.

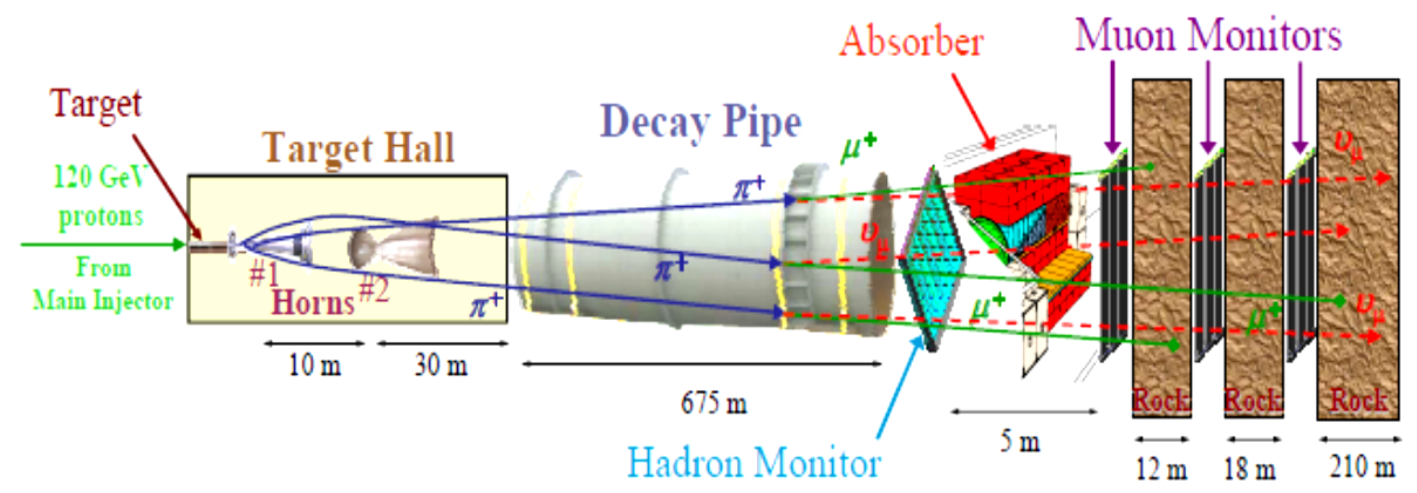

Figure 3.1. A schematic of the NuMI beamline (not drawn to scale). Reprinted from [52], Copyright (2005), with permissions from R. Zwaska.

\subsubsection{THE PROTON BEAM}

The protons that interact strongly with the NuMI production target undergo a number of acceleration stages to reach the operational mode of $120 \mathrm{GeV} / \mathrm{c}$. First, the Fermilab Linac (Linear Accelarator) accelerates negative hydrogen ions to an energy of $400 \mathrm{MeV}$ [55], [56]. The Booster, a fast-cycling synchrotron, accepts the ions from the Linac and the ions are circulated counterclockwise until they reach a maximum energy of $8 \mathrm{GeV}$ [56]. The MI-8 line transfers the $8 \mathrm{GeV}$ protons to the Main Injector ring, which is a synchrotron that accelerates the protons to a final momentum of 120 $\mathrm{GeV} / \mathrm{c}[57]$. The Booster can deliver up to six batches of protons to the Main Injector 
[58]. A batch consists of a proton beam that has been accelerated and extracted by the Booster in one cycle. During the Tevatron running, five or six of the batches are extracted to NuMI. Once the Main Injector has been fed all six batches, the protons are accelerated and then transferred to NuMI. The proton beam is delivered to NuMI every 2.2 seconds where the width of the NuMI beam spill is $\sim 8$ microseconds $(\sim 10$ microseconds) when containing five (six) of the Booster batches.

\subsubsection{THE NuMI APPARATUS}

As shown in Figure 3.1, the NuMI beamline is a multi-stage apparatus. The different components of the apparatus are described below.

\section{The Production Target}

A $120 \mathrm{GeV} / \mathrm{c}$ proton beam is focused onto a graphite target, where Figure 3.2 shows a schematic diagram of the NuMI production target. The proton beam has a maximum power of $404 \mathrm{~kW}$ [56]. Before the protons collide with the target, the proton beam propagates through a baffle aperture system that protects the magnetic horns from the proton beam pulses, because kilowatt proton beams can damage most materials very quickly [59]. The baffle acts as a collimator which reduces the beam spot to $\sim 1 \mathrm{~mm}$ in diameter at the target [58], [60]. The production target is composed of 47 fins which are target segments made of graphite material.

Each fin is $2 \mathrm{~cm}$ long, with $0.3 \mathrm{~cm}$ spacing between each segment. This gives the target a total length of $95.38 \mathrm{~cm}$ which corresponds to about 2 nuclear interaction lengths or 4 radiation lengths [55], [61], [60]. The production target is water cooled at both the top and bottom and is contained in an aluminum vacuum can with beryllium windows [58]. The target and cooling tube are electrically isolated so that the signal from the charged particles in an extra 


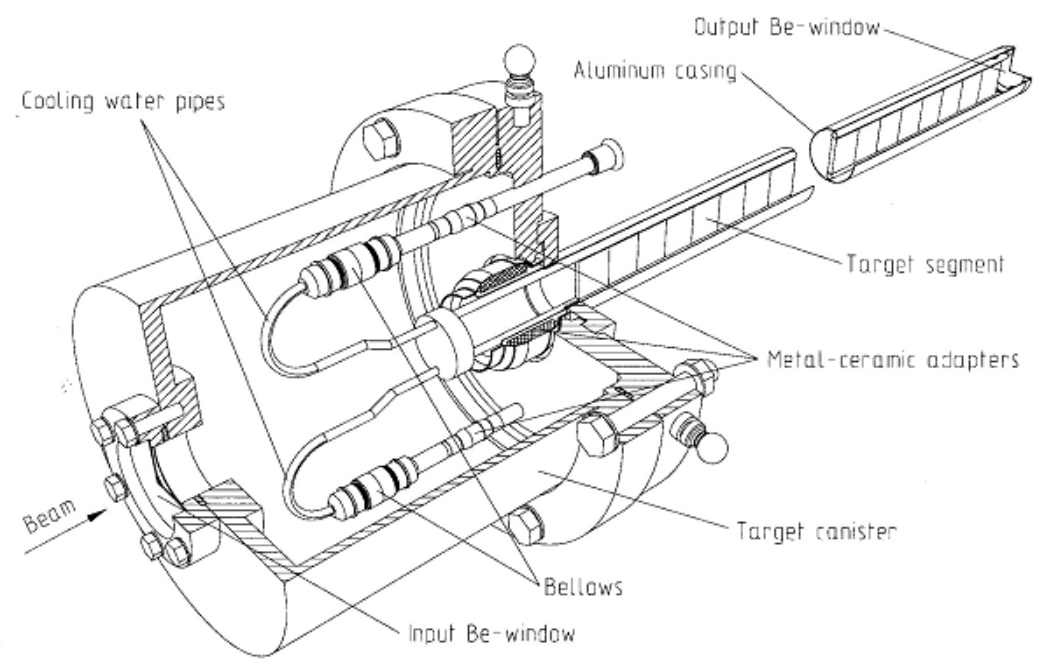

Figure 3.2. The NuMI production target and target vacuum canister, where the Target Segments are most commonly referred to as fins. The target is mounted on a raildriver system with a maximum longitudinal travel length of $2.5 \mathrm{~m}$. Reprinted from Reference [53], Copyright (2002).

$48^{\text {th }}$ fin can served as a beam monitor called the Budal monitor [58]. The Budal monitor is used to track the relative position of the target and the primary proton beam [61], [53]. Another important feature of the NuMI target is that its position can be moved along the beam axis in order to produce beams with different energy spectrums.

\section{The Magnetic Horns}

The hadrons produced in the target travel through the magnetic focusing horns where the horns' inner conductors are shaped like a parabola (see Figure 3.3). When the horns are supplied with a maximum current of $200 \mathrm{kA}$, a toroidal magnetic field of $30 \mathrm{kG}$ is produced [58]. The generated magnetic field acts as a lens where the focal length is proportional to the scattered hadron momentum [56]. The target's position relative to the first magnetic horn can be adjusted such that a particular momentum range of charged particles is focused by the magnetic field. The focused hadrons safely pass through the central aperture of 
the second magnetic horn. The hadrons with momentum values and angles out of focus are refracted by the second magnetic horn. In addition, the polarity of the current determines the charge of the particles that curve towards the direction of the NuMI beamline. For the data presented in this thesis, the target-horn system was in the configuration called the Forward Horn Current (FHC) mode. In the FHC mode, positive charged particles are focused by the magnetic horns to produce a $\nu_{\mu}$-beam.

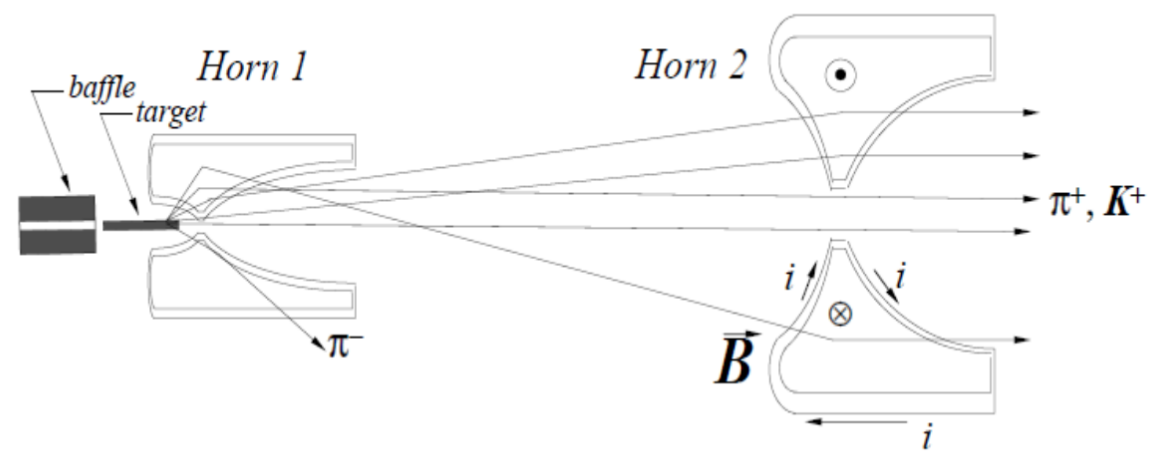

Figure 3.3. A schematic diagram of NuMI two focusing magnetic horns. Reprinted from Reference [55], Copyright (2005).

\section{The Decay Pipe}

The hadrons selected by the focusing horns make their way to the decay volume (commonly referred to as the decay pipe) as shown in Figure 3.1. Most of these hadrons are primarily non-interacting protons, pions and a small contribution from kaons [56]. The decay volume is a cylindrical steel pipe that is 675 meters long with a diameter of 2 meters and filled with 1 atm helium gas to reduce pion absorption. [58], [62]. The length of decay pipe corresponds to the decay length of a $10 \mathrm{GeV}$ pion. The charged mesons decay into neutrinos and muons in the decay pipe. 


\section{The Hadron Monitors and Absorber}

The NuMI hadron monitors and absorber are situated downstream of the decay pipe. The $1 \times 1 \mathrm{~m}^{2}$ hadron monitor is a $7 \times 7$ pixels ion chamber filled with helium gas. The purpose of the hadron monitor is to measure the position and intensity of the proton beam at the end of the decay volume [63]. All protons from the primary beam and mesons that do not decay are stopped by the hadron absorber, which is composed of aluminum, steel and concrete. The hadron absorber sits directly downstream of the hadron monitor.

\section{The Muon Monitors}

Only the muons and neutrinos remain in the NuMI beamline downstream of the hadron absorber. For the NuMI Low Energy Run Period, only three muon monitors are positioned downstream of the hadron absorber in the NuMI facility. In the summer of 2013 a fourth muon monitor was installed and will be available for the Medium Energy Running of the NuMI beamline. Figure 3.4 shows a schematic diagram of the NuMI hadron and muon monitors apparatus setup. The $2 \times 2 \mathrm{~m}^{2}$ muon monitors are $9 \times 9$ pixels helium-filled ion chambers which are positioned in alcoves embedded in dolomite rock [63]. As the muons propagate downstream of NuMI, each successive muon monitor detects muons of increasingly higher energy. The muons which stopped before the muon monitor apparatus, originate from low energy mesons that were produced from the $p C$ collisions. Hence, the muon monitors observe the muons whose parents are the higher energy mesons. By comparing the muon rates at the various alcoves, information about the kinematics of the mesons that decay to produce neutrinos is obtained. This information can be used to constrain the model of the neutrino beam. 
The muons and neutrinos that have survived after passing through the most downstream alcove must then traverse an additional 210 meters of rock before entering the MINOS Underground Near Detector Hall. The muons that originate from the meson decay products range out in the rock. The neutrinos continue to move towards the detector hall.

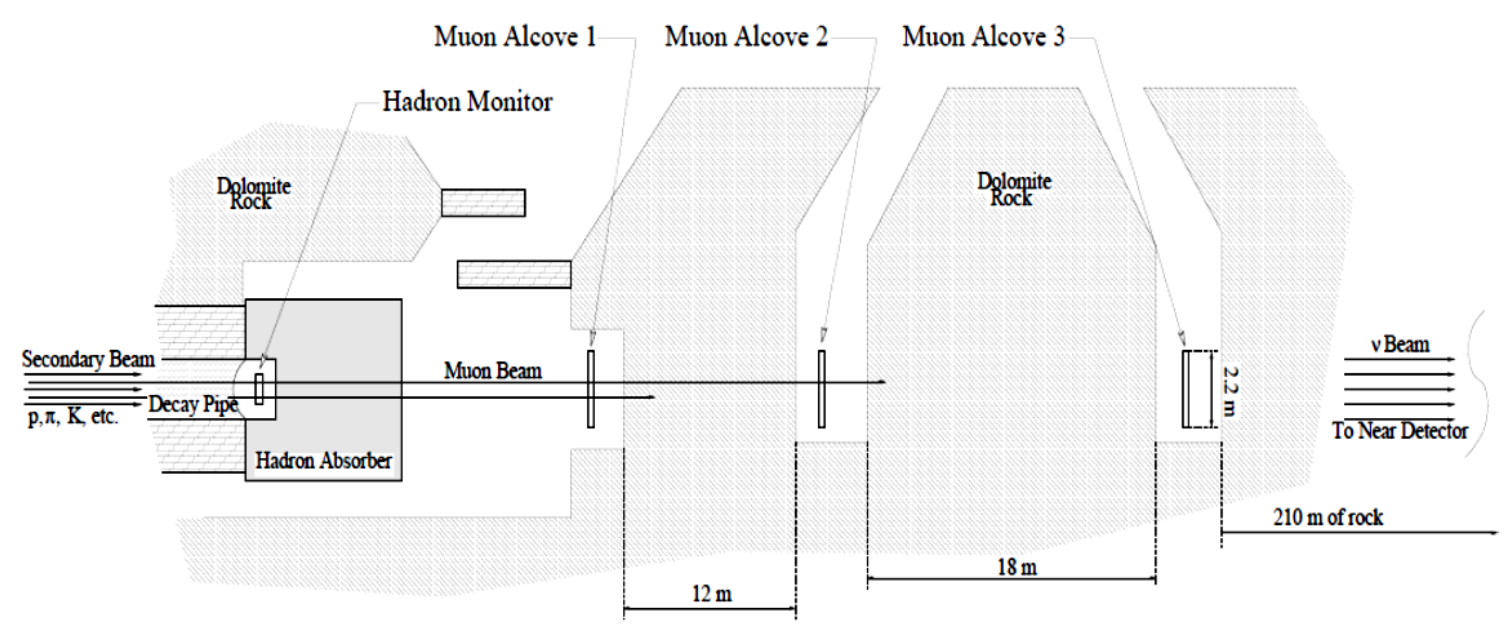

Figure 3.4. A schematic picture of th NuMI Hadron and Muon Monitors before the installation of the fourth muon alcove. Reprinted from Reference [53], Copyright (2006) with permissions from Elsevier.

\subsection{THE MINER $\nu$ A DETECTOR}

This section gives a description of the various components of MINER $\nu \mathrm{A}$ and Figure 3.5 shows a schematic diagram of the detector. The main part of the MINER $\nu \mathrm{A}$ detector is categorized into two separate regions, the inner detector (ID) and the outer detector (OD). The ID is assembled into four sub-regions along the beam axis: the nuclear targets region, an active tracker region, downstream electromagnetic calorimetry (ECAL), and downstream hadronic calorimetry (HCAL). The OD is composed 
mostly of frames of steel and serves as the side hadronic calorimetry. Upstream of the detector, a veto wall is placed to tag muons coming from the rock. Between the veto wall and the main MINER $\nu \mathrm{A}$ detector, a cryogenic vessel filled with liquid helium is situated.

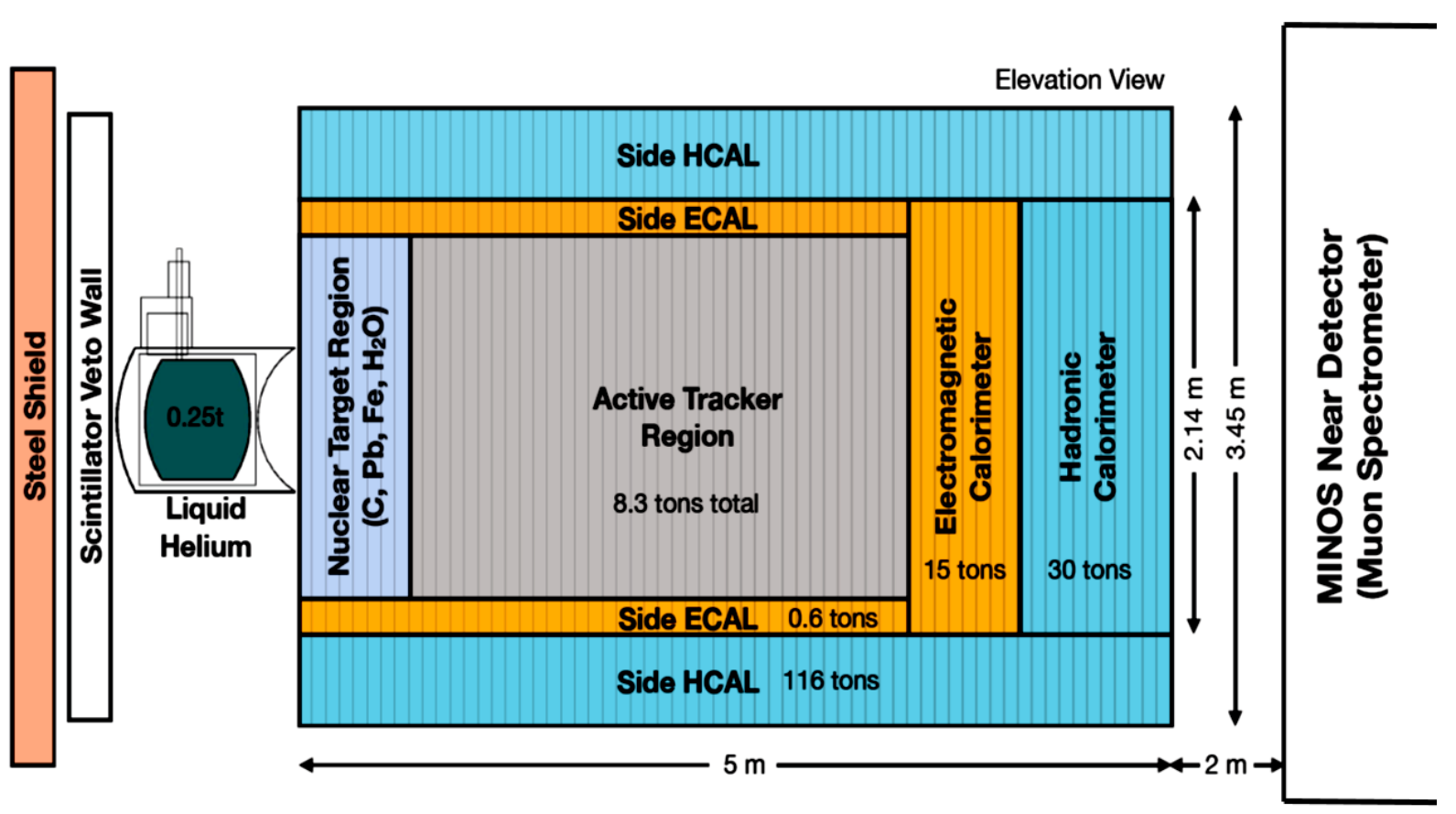

Figure 3.5. A schematic diagram of the MINER $\nu \mathrm{A}$ detector as shown from the top view. Reprinted from [51], Copyright (2013).

\subsubsection{INNER DETECTOR}

MINER $\nu \mathrm{A}$ is composed of 120 modules that are stacked along the direction of the beam. The shape of each module is a regular hexagon. The ID consists of four types of modules which are mounted in outer detector frames: tracking, electromagnetic calorimetry, hadronic calorimetry, and passive nuclear targets modules. Figure 3.6 shows the front view of a tracking module mounted in an outer detector frame. The various composition of the ID subdetectors are described below. 


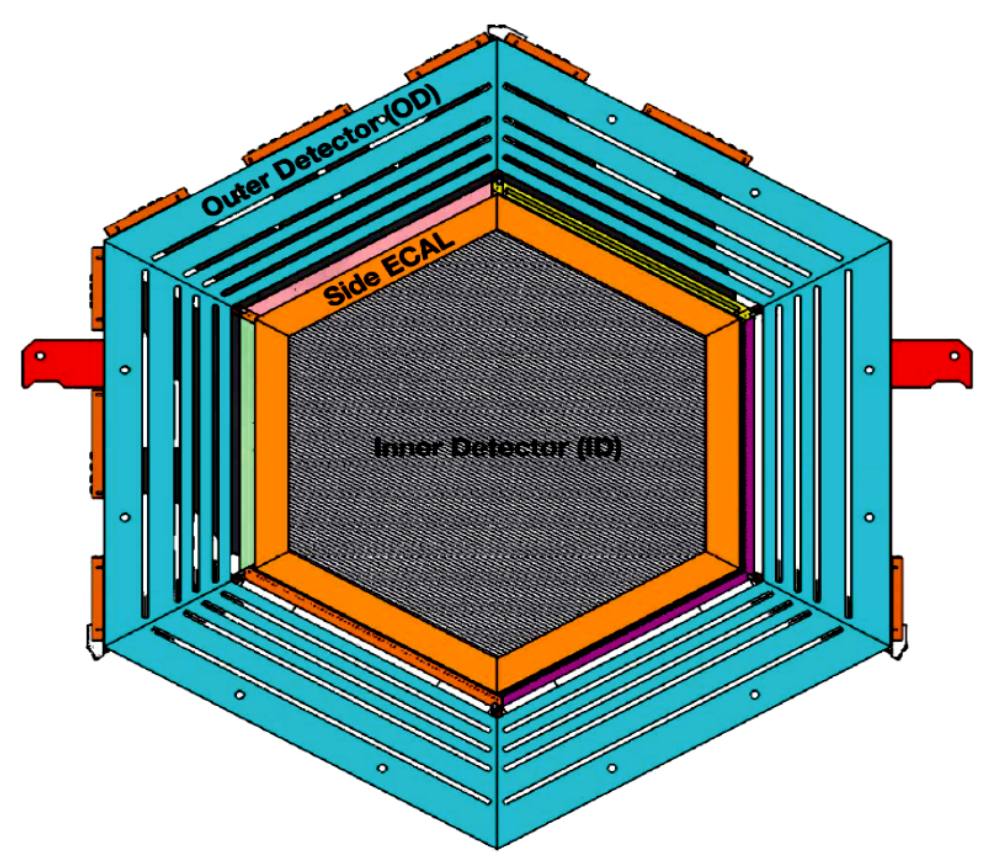

Figure 3.6. A schematic diagram of a tracking module mounted in an outer detector frame. Reprinted from [51], Copyright (2013).

\section{The Inner Detector Planes}

An ID plane is composed of 127 polystyrene triangular scintillator strips, that are arranged in a saw-tooth pattern (see Figure 3.7). Section 3.2.4 contains descriptive details about the scintillator strips. The strips are glued together with 3M-DP190 translucent epoxy to form a hexagon plane with an apothem of $1.07 \mathrm{~m}$ and a width of $2.1 \mathrm{~m}$. On both the downstream and upstream sides of the plane, sheets of GE polycarbonate (Lexan) are glued with 3M-DP190 gray epoxy to make them light tight. Any additional light leaks are controlled with black PVC electrical tape, where the light leaks are mainly due to gaps in the Lexan sections. Table 3.2.1 shows the percentage of various elements that make up the scintillator plane, where the measured areal density is $2.02 \pm 0.03 \mathrm{~g} / \mathrm{cm}^{2}$.

MINER $\nu \mathrm{A}$ uses a right-handed coordinate system as shown in Figure 3.8. The coordinate system is defined such that the z-axis is along the direction of the beam, 


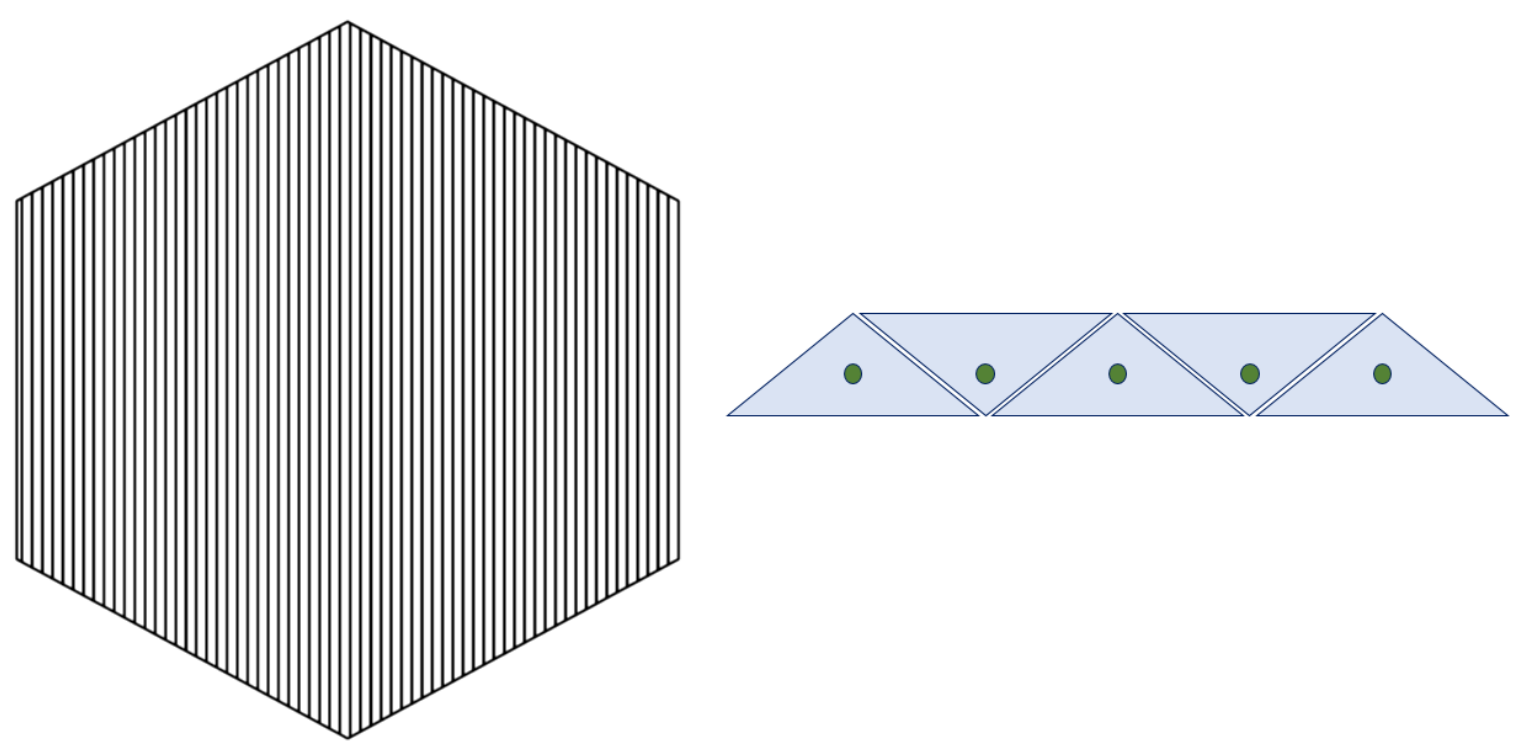

Figure 3.7. A schematic diagram of the ID scintillator plane orientated in the X-view. The left diagram shows the plane from the front view. The right figure shows the cross section of the triangular scintillator strips. Each strip contains a WLS fiber.

Table 3.1. The Element Composition of the Scintillator Plane by Mass Percentage.

\begin{tabular}{l||c}
\hline \hline Element & Percentage (\%) \\
\hline \hline Hydrogen & 7.42 \\
Carbon & 87.6 \\
Oxygen & 3.18 \\
Aluminum & 0.26 \\
Silicon & 0.27 \\
Chlorine & 0.55 \\
Titanium & 0.69 \\
\hline \hline
\end{tabular}

where the NuMI beamline is directed into the Earth at an angle of $58 \mathrm{mrad}$. The $\mathrm{y}$-axis points upward, away from the center of the Earth. The x-axis is horizontal and points to the left as viewed from the beam. The $x-y$ origin is placed in the center of the detector.

The hexagonal planes are oriented into the three different views, $\mathrm{X}$, $\mathrm{U}$, or $\mathrm{V}$. In the X-view, the plane's strips are aligned parallel to the y-axis. A U or V plane 


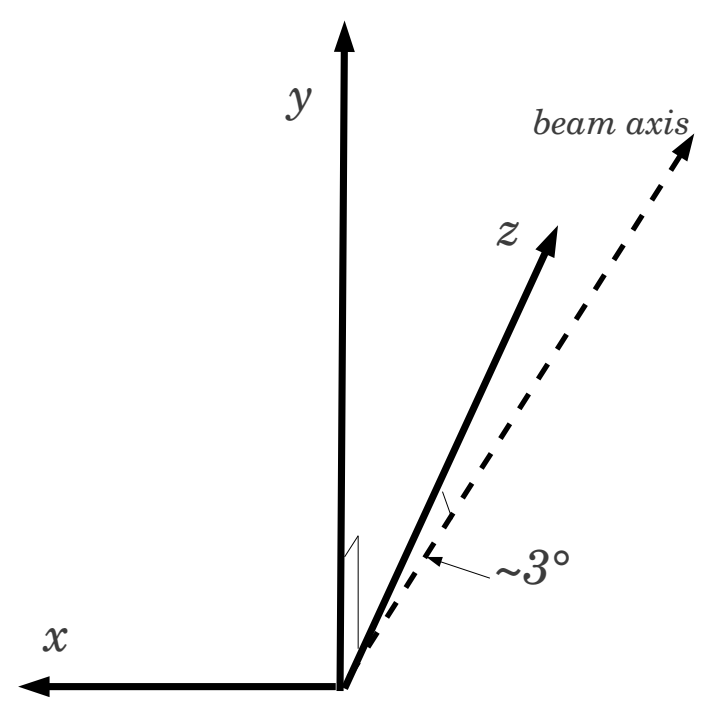

Figure 3.8. A schematic diagram of the MINER $\nu$ A coordinate system.

corresponds to the rotation of a plane +60 or -60 degrees in the XY-plane relative to the x-axis. The three different views allow for the reconstruction of three-dimensional objects such as tracks.

\section{The Tracker Region}

The tracker region consists of 62 tracking modules, which each contains two hexagonal, scintillator planes. As shown in Figure 3.6, the downstream side of each plane is bordered with a $0.2 \mathrm{~cm}$ thick lead collar. The width of the lead collar is $15 \mathrm{~cm}$. The lead collar starts out at approximately $90 \mathrm{~cm}$ for the center of a plane and extends to the plane's outer border. The lead collar region of the tracking planes served as the side electromagnetic calorimetry as shown in Figure 3.5. The planes are arranged in either the UX or VX orientation. The modules are stacked in alternating UX/VX orientations along the direction of the beam. 


\section{The Downstream Electromagnetic Calorimetry}

The ECAL region contains 10 modules which are assembled similar to the tracking modules. An ECAL module has a $0.2 \mathrm{~cm}$ thick lead sheet that covers the entire downstream side of a plane. A transition module lives upstream of the ECAL modules. For the transition module, the upstream plane is bordered with a lead collar, while the downstream plane is covered with a lead sheet. The purpose of the transition module is to allow for a lead absorber to sit upstream of each ECAL plane.

\section{The Downstream Hadron Calorimetry}

There are 20 modules that make up the HCAL region. Unlike the tracking and ECAL modules, the HCAL module consists of only one scintillator plane and a 2.54 $\mathrm{cm}$ thick hexagonal steel plane. The steel plane is assembled downstream of the scintillator plane. The planes in consecutive modules exhibit the XVXU repeating pattern.

\section{The Nuclear Targets Region}

The nuclear targets region contains 22 tracking modules, 5 solid passive targets, and a water target. Figure 3.9 shows a schematic diagram of the nuclear targets region. There are four tracking modules before the first target and between each target, except for the fourth and fifth passive solid targets, which are separated by two tracking modules. The tracking modules are necessary to obtain efficient vertex, track, and shower reconstruction for particles that originate from neutrino interactions within a passive target. Similar to the tracking modules, each solid target module is shaped as a hexagon and mounted in an outer detector steel frame. The OD frames are discussed in more details in the subsection 3.2.2. Except for the fourth target, each solid target is sectioned into different nuclei, where the sections are arranged in various orientations as indicated in Figure 3.9. The primary purposes of this arrangement 
allow for producing precision targets acceptance studies and measuring the cross sections in form of ratios to partially remove the acceptance and z-dependence effects.

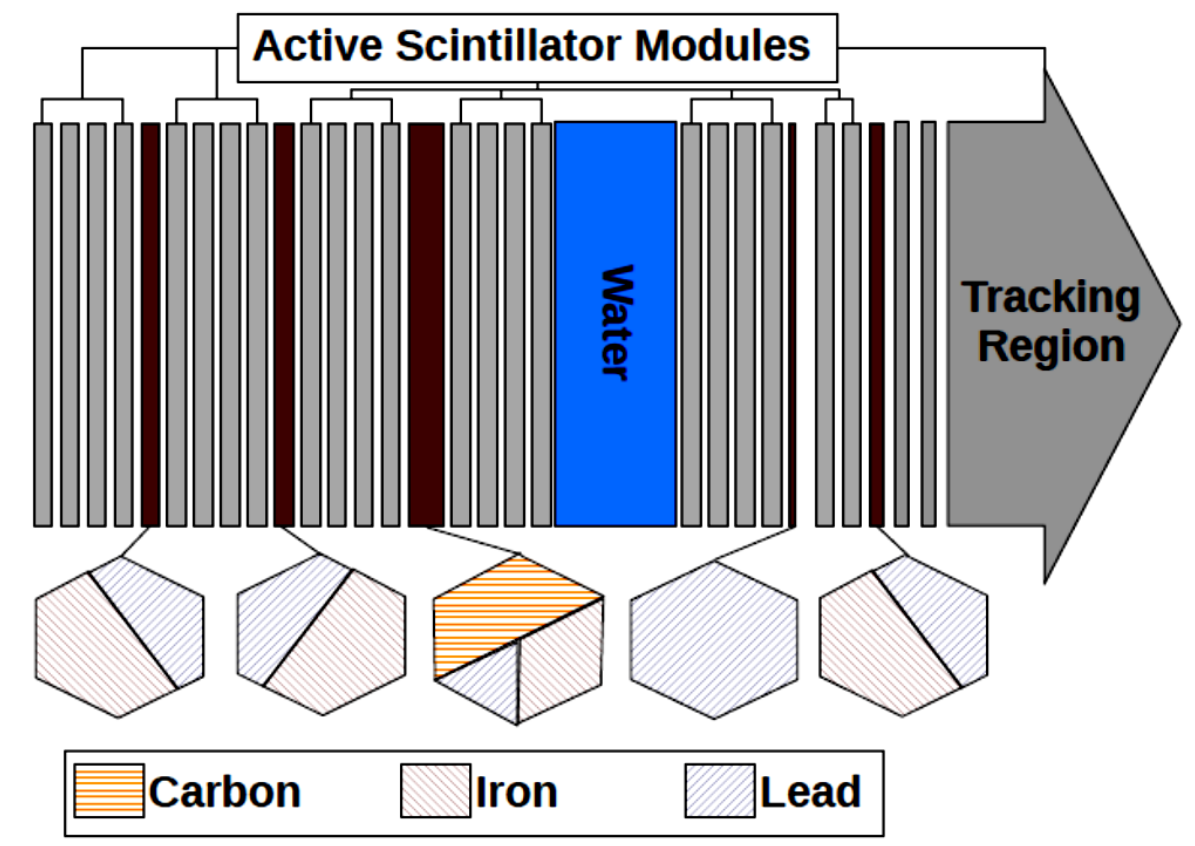

Figure 3.9. A schematic diagram of the nuclear targets region in the MINER $\nu \mathrm{A}$ experiment, where the liquid cryotarget vessel is excluded. The Targets 1, 2, and 5 are composed of the iron and lead nuclei, Target 3 is composed of carbon, iron, and lead, and Target 4 is composed only of lead. The water target was not installed at the beginning of the LE run period. Reprinted from [53], Copyright (2013) with permissions from B. Tice.

For Targets 1, 2, and 5, the iron and lead regions are divided diagonally on a $20.5 \mathrm{~cm}$ offset from the center of the target. Target 3 is composed of carbon, iron, and lead regions, where each region occupies $\frac{1}{2}, \frac{1}{3}$, and $\frac{1}{6}$ of the tri-target's area, respectively. The carbon is approximately 3 times thicker than the iron and lead, which is necessary so that the nuclear interaction length is approximately the same for all three materials. Since, the materials are aligned at the front of the module, this leads to an air gap between the iron/lead sections and the tracking module that is directly downstream of the target. As shown in the Table 3.2.1, the fourth target is composed only of lead and is the thinnest target. It serves for studying the electromagnetic induced interactions from particles originating from the passive targets. In addition, the thickness of the 
solid targets also differ. The thickest targets are placed in the upstream region and the thinnest targets lived in the downstream region. The Table 3.2.1 gives the material and geometrical composition of each target.

Table 3.2. The Nuclear Targets Material and Geometrical Description.

\begin{tabular}{l|c|c|c}
\hline \hline Target Number & Nucleus & Fiducial Mass $(\mathrm{kg})$ & Thickness $(\mathrm{cm})$ \\
\hline Target 1 & Iron & 322 & $2.567 \pm 0.006$ \\
& Lead & 263 & $2.578 \pm 0.012$ \\
\hline Target 2 & Iron & 321 & $2.563 \pm 0.006$ \\
& Lead & 263 & $2.581 \pm 0.016$ \\
\hline Target 3 & Carbon & 158 & $2.573 \pm 0.004$ \\
& Iron & 107 & $2.563 \pm 0.004$ \\
& Lead & 160 & $7.620 \pm 0.005$ \\
\hline Target 4 & Lead & 225 & $0.795 \pm 0.005$ \\
\hline Target 5 & Iron & 162 & $1.289 \pm 0.006$ \\
& Lead & 134 & $1.317 \pm 0.007$ \\
\hline \hline
\end{tabular}

The water target is positioned between the third and fourth solid targets. The data collected for the water target was not used for studying the neutrino interactions on nuclei in this analysis, and will not be futher discussed.

\subsubsection{OUTER DETECTOR}

The OD is composed of hexagonal steel frames, which provides the structural support system of the ID modules and serves as the side hadronic calorimetry as shown in Figure 3.5. The OD frames that support the tracking and ECAL modules are $3.49 \mathrm{~cm}$ thick, whereas the frames in the HCAL region are $3.81 \mathrm{~cm}$ thick. Each frame is composed of six sections called towers as shown in Figure 3.6, where a tower is approximately $56 \mathrm{~cm}$ wide at its halfway position. A tower is also commonly referred to as a group of OD doublets which occupy 4 slots in each section of the OD steel frame. Each slot has a different length which corresponds to the span to the tower's outer edges. The slot is instrumented with a pair of $2.54 \times 2.54 \mathrm{~cm}^{2}$ rectangle scintillator strips called an OD bar or an OD doublet as shown in Figure 3.10. 


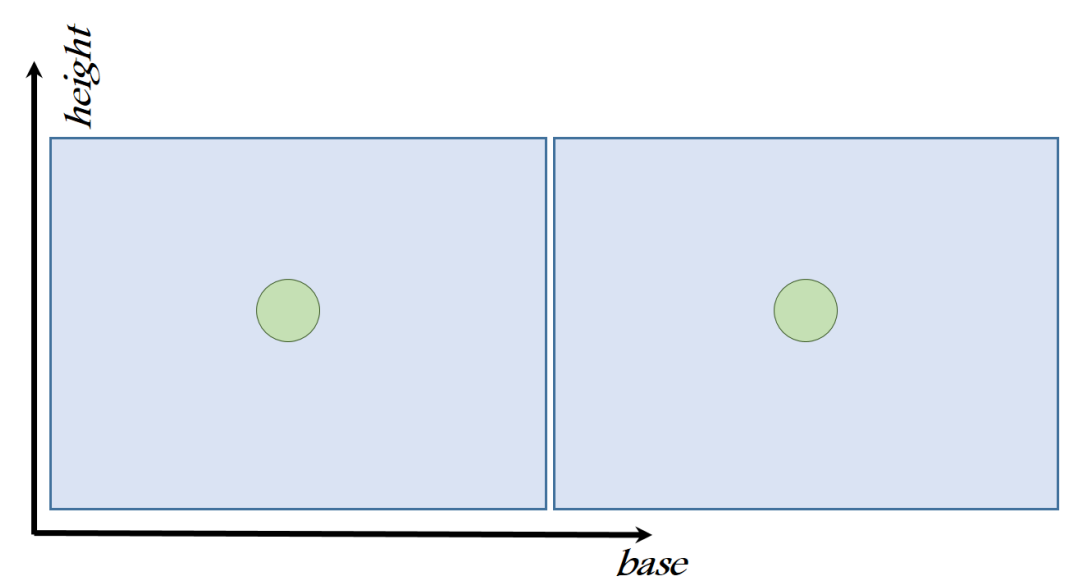

Figure 3.10. A schematic diagram of the cross section of an OD bar. An OD bar is composed of two rectangular scintillator strips with wavelength shifting fiber running through each strip.

Starting from the bar closest the inner edge of the OD frame, the length of the bars are as the following: $52.66,56.38,60.16$, and $66.50 \mathrm{~cm}$. The scintillator strips are described in the subsection 3.2.4. The space between the slots are not equal. The distance between the inner edge of an OD frame to each slot is 6.35, 15.24, 23.50, and $37.47 \mathrm{~cm}$. The OD also performs as a crude tracking detector due to the orientation and separation distance between the bars and the spacing between frames.

\subsubsection{UPSTREAM REGION}

As mentioned previously, the region upstream of MINER $\nu \mathrm{A}$ contains a veto wall followed by a cryogenic helium target. The veto wall consists of two layers of iron and scintillator paddles utilized for absorbing and identifying charged particles that enter the front face of the MINER $\nu \mathrm{A}$ detector. The neutrinos in the NuMI beam can undergo both the charged and neutral current interactions with the rock before entering the Near Detector Hall. The iron slab is useful for stopping the low energy hadrons from contaminating the cryotarget and main detector. The scintillator paddles detect muons from the neutrino $\mathrm{CC}$ interactions with the rock. These muons 
are called rock muons. The veto wall was fully installed and instrumented towards the end of the LE run period. The detectors in the upstream region of MINER $\nu \mathrm{A}$ are excluded from this analysis, therefore will not be discussed further. The first four scintillator planes at the front face of the nuclear targets region are used for identifying rock muon events, where the rock muons have deposited energy in a minimum of 170 scintillator planes.

\subsubsection{THE SCINTILLATOR STRIPS}

The ID triangular strips and the OD rectangular strips are extruded from Dow Styron $663(\mathrm{~W})$ polystyrene $\left((\mathrm{CH})_{n 8}\right)$, scintillator. The scintillator is doped with 2,5-diphenyloxazole (PPO or $\mathrm{C}_{15} \mathrm{H}_{11} \mathrm{NO}$ ) and 1,4-bis (5-phenyloxazol-2-y1) benzene (POPOP or $\mathrm{C}_{24} \mathrm{H}_{16} \mathrm{~N}_{2} \mathrm{O}_{2}$ ). The dopants, PPO and POPOP are approximately $1 \%$ and $0.03 \%$ of the weight of the scintillator. The dopants cause the ionization loss of the charged particles in the scintillator to convert into wavelengths of light [55]. The strips are coated with a layer of white reflective paint that is a diffuse reflector and composed of polystyrene and $\mathrm{TiO}_{2}$, where the $\mathrm{TiO}_{2}$ makes up about $15 \%$ of the weight of the paint.

The triangular strips have a width at the base of $3.3 \pm 0.05 \mathrm{~cm}$ and a height of $1.7 \pm 0.05 \mathrm{~cm}$. The cross section of a rectangular strip in an OD bar are $1.9 \pm 0.05$ $\mathrm{cm}$ base and $1.66 \pm 0.05 \mathrm{~cm}$ height as indicated in Figure 3.10. Each strip has a $0.26 \pm 0.02 \mathrm{~cm}$ diameter hole positioned halfway above its base. The hole runs the entire length of the strip and is filled with a 175 ppm Y-11 doped, S-35 multicade wavelength shifting (WLS) fiber, where optical epoxy is utilized to position the fiber in the holes. The WLS fiber collects the light from the scintillator strip, shifts it to green, and propagates the light out of the strip. 
Only one end of the WLS fiber is read out. The unread end of each fiber is mirrored in order to maximize the light collection. The mirroring procedure is as the following:

1. The end of the fiber is prepared for polishing using the ice-polishing technique.

2. A $2500 \AA$ thick reflective coating of pure aluminum is sputtered at the end of the fiber and the coating serves as the mirrors.

3. A layer of Red Spot UV Epoxy is applied for protecting the mirrors.

The read-out end of the WLS fiber extends the length of the strip by $\sim 8 \mathrm{~cm}$ and is positioned in an optical connector (Fujikura-DDK connectors). Each DDK connector groups eight fibers. The DDK connector attaches a cable of clear optical fibers to the WLS fibers. The purpose of the clear optical fibers (jumper cables) is to transmit light from the WLS fibers to the photomultiplier tube (PMT).

\subsubsection{THE PHOTOMULTIPLIER TUBE AND OPTICAL BOX}

The clear optical fibers are connected to a multi-anode PMT with the model number of H8804MOD-2 and manufactured by Hamamatsu Photonics. The scintillation light collides with the PMT's photocathode which made of Bialkai material and where photoelectrons are emitted from the photocathode via the photoelectric effect. The photoelectrons are amplified in a series of 12 dynode stages, where approximately $10^{5}$

to $10^{6}$ photoelectrons per signal are expected at the anode stage [55]. Each PMT contains an array of $8 \times 8$ pixels, which are mapped onto a $2 \times 2 \mathrm{~cm}^{2}$ grid. There are 64 pixels per PMT, thus eight sets of eight fibers can connected to a PMT. The PMT lives in a light-tighten cylindrical box made out steel as shown in Figure 3.11. 


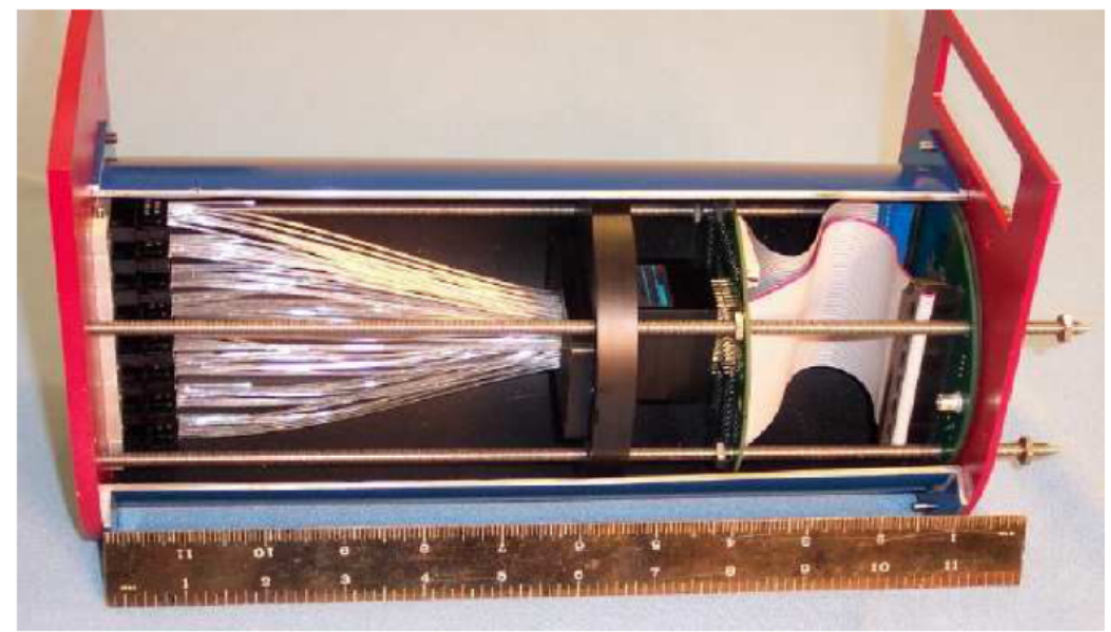

Figure 3.11. A picture of the components that make up the MINER $\nu \mathrm{A}$ PMT optical box. The PMT box is displayed from the back-end to the front-end. The ODU is positioned between the box's back-end and the black disc which mounts the fiber cookie. The PMT is mounted on a base circuit board and the cylindrical rods are used to ensure alignment.

An optical decoder unit (ODU) frame is placed at the back face of each PMT box. Eight sets of jumper cables connect to the ODU ports that extend out of the steel optical box. An ODU is a set of $8 \times 8$ clear optical fibers that transmit light from the cables to the PMT. As shown in Figure 3.11, the PMT is mounted to a base circuit board, which provides the signal routing to the front-end electronics. A fiber cookie is positioned at the opposite end of the PMT. The cookie maps the clear fibers into the PMT, where it ensures that the signals from adjacent strips do not propagate to adjacent pixels of the PMT. This is required to eliminate some of the cross-talk between the PMT channels. MINER $\nu \mathrm{A}$ is instrumented with a total of $507 \mathrm{PMTs}$ which produce approximately 32,000 channels for readout. 


\subsubsection{READOUT ELECTRONICS AND THE DAT ACQUI- SITION SYSTEM}

A Front End Board (FEB) is attached to front face of the PMT optical box and reads out analog signals from the PMT, where the FEB is controlled by a Spartan 3E Field-Programmable Gate Array (FPGA) chip. The FEB applies high voltage to the PMT dynodes using an on-board Cockroft-Walton (CW) generator. The charge from the PMT is stored and digitized in its six Application-Specific Integrated Circuit chips, better known as TriP-t chips. The TriP-t chip integrates the signal charge from the PMT over a time window (gate), where the standard gate length is $\sim 16 \mu$ s. Each TriP-t chip carries 32 channels. FEB is mounted with six TriP-t chips, where four operates in the high and medium gain and two in low gain. The high gain channels defined the discriminator threshold. When a charge in the channel surpasses the discriminator threshold, the TriP-t chip integrates the charge and stores the hit. The integration time after the discriminator fires is $\sim 150 \mathrm{~ns}$ with a reset time window of $\sim 20$ ns.

Up to ten FEBs are daisy-chained together using UTP ethernet networking cables. The FEB chain is connected at both ends to a Chain Readout Controller (CROC), a custom made VME module. A CROC can support up to four chains of FEBs, where each CROC channel has a $6 \mathrm{kB}$ of dual-port memory for the FEB chain. The VME modules are housed in a VME crate which contains a CAEN V2718 crate controller. In addition to the CROCs, a CROC Interface Module (CRIM) is also installed in the VME crate. The CRIM sends the CROC timing and trigger commands, where the CRIM receives information from MINOS timing system and the Main Injector via the MINER $\nu$ A Timing Module (MvTM). Up to four CROCs can be plugged into

a CRIM. MINER $\nu$ A has 2 VME crates, 2 CRIMs, 15 CROCs, and 509 FEBs. The VME crates communicate with a computer running the DAQ program. For more 
information on the MINER $\nu \mathrm{A}$ Readout Electronics and Data Acquisition System see Reference [64].

\subsection{THE MINOS NEAR DETECTOR}

The MINOS experiment is an on-axis neutrino oscillation experiment with a near detector and a far detector which is located approximately $735 \mathrm{~km}$ away in Soudan, Minnesota [12]. The MINER $\nu \mathrm{A}$ detector is located about 2 meters upstream of the MINOS near detector. The MINOS near detector (here-forth referred to as MINOS) is used for analyzing muons that exit the MINER $\nu \mathrm{A}$ detector.
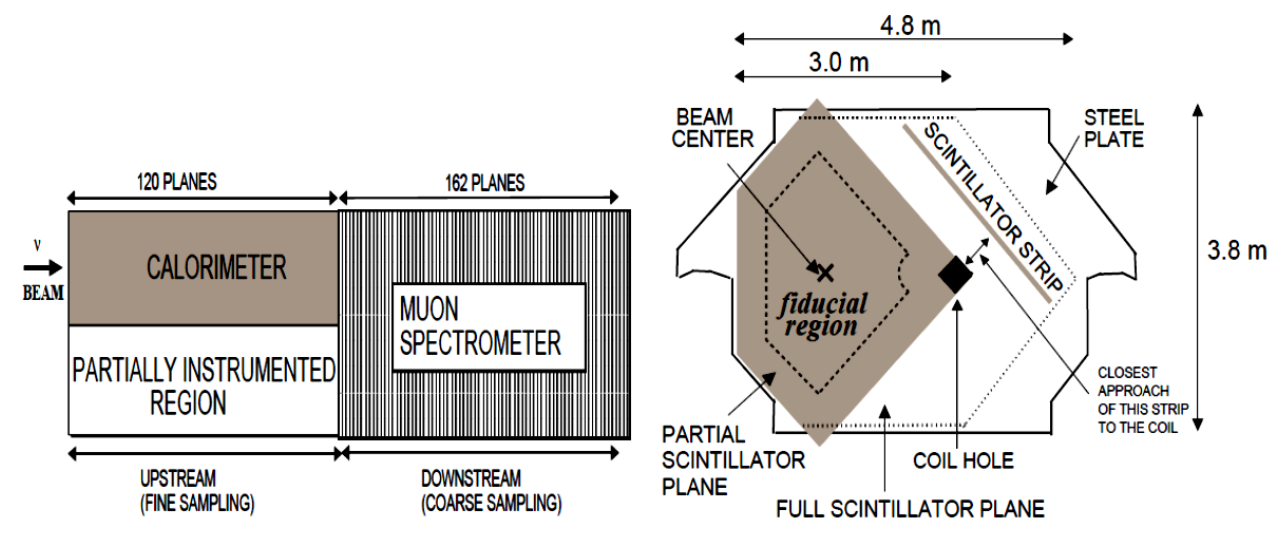

Figure 3.12. The MINOS near detector. The left (right) shows a top (beam) view of the detector. Reprinted from [65], Copyright (2010) with permissions from the MINOS Collaboration.

MINOS is a tracking calorimetry detector which has a calorimetry region and muon spectrometer region as shown in Figure 3.12. The detector is assembled with alternating magnetized steel planes and plastic scintillator planes, where the steel and scintillator planes are $2.54 \mathrm{~cm}$ and $1 \mathrm{~cm}$ thick, respectively. The scintillator planes are composed of $4.1 \mathrm{~cm}$ wide strips made out of the same compound mixture as the MINER $\nu \mathrm{A}$ scintillator strips. The calorimetry region, the upstream region of the 
detector is composed of 120 planes, where each steel plane has a plastic scintillator plane attached to its upstream edge. The MINOS planes are rotated 45 degrees clockwise or counterclockwise with respect to the vertical axis. In this region, the planes are either partial-instrumented or fully-instrumented, where the partial-instrumented planes only covers a fraction of the steel plane. The muon spectrometer region consists of 162 planes, where every fifth steel plane is affixed to a fully-instrumented scintillator plane.

MINOS serves as a muon toroidal magnetic spectrometer for the MINER $\nu \mathrm{A}$ experiment. The spectrometer has a magnetic field of $1.3 \mathrm{~T}$, where the field is generated by a current of $40 \mathrm{kA}$. The polarity of the current can be adjusted such that either the negative or positive charged particles are more likely to be contained in the detector. The polarity of the current depends on the NuMI beam mode, the Forward Horn Current (FHC) or the Reverse Horn Current (RHC).

\subsection{THE DATA RUN PERIOD}

The main part of MINER $\nu \mathrm{A}$ was fully installed and instrumented by the end of March 2010. However, MINER $\nu$ A has been taking data since the winter of 2009 as shown in Figure 3.13, where this data period is known as the Low Energy Run. The Low Energy (LE) Run refers to the NuMI beamline operating in a configuration such that there is a $10 \mathrm{~cm}$ separation distance between the NuMI production target and magnetic horns with a current of $185 \mathrm{kA}$. For the first four months of the run period, data was collected for a partially instrumented MINER $\nu \mathrm{A}$ detector and the NuMI beamline was in the RHC focusing mode. In the RHC mode, the NuMI beam is predominately $\bar{\nu}_{\mu}$ particles. This period corresponds to the Frozen Detector Era. As mentioned earlier, this analysis analyzes the data where the NuMI beamline was in the FHC configuration ( $\nu_{\mu}$-mode). Thus the data collected during the Frozen Detector Era as well as all other periods when the NuMI was in the $\bar{\nu}_{\mu}$-mode is irrelevant to 
this dissertation. In addition, during the $\mathrm{LE}$ run, MINER $\nu \mathrm{A}$ recorded data when the NuMI beamline operated in special configurations, when the separation distance between the target and horns was not $10 \mathrm{~cm}$ and/or the supplied current was not 185 kA. The data collected for the special runs were not analyzed in this thesis.

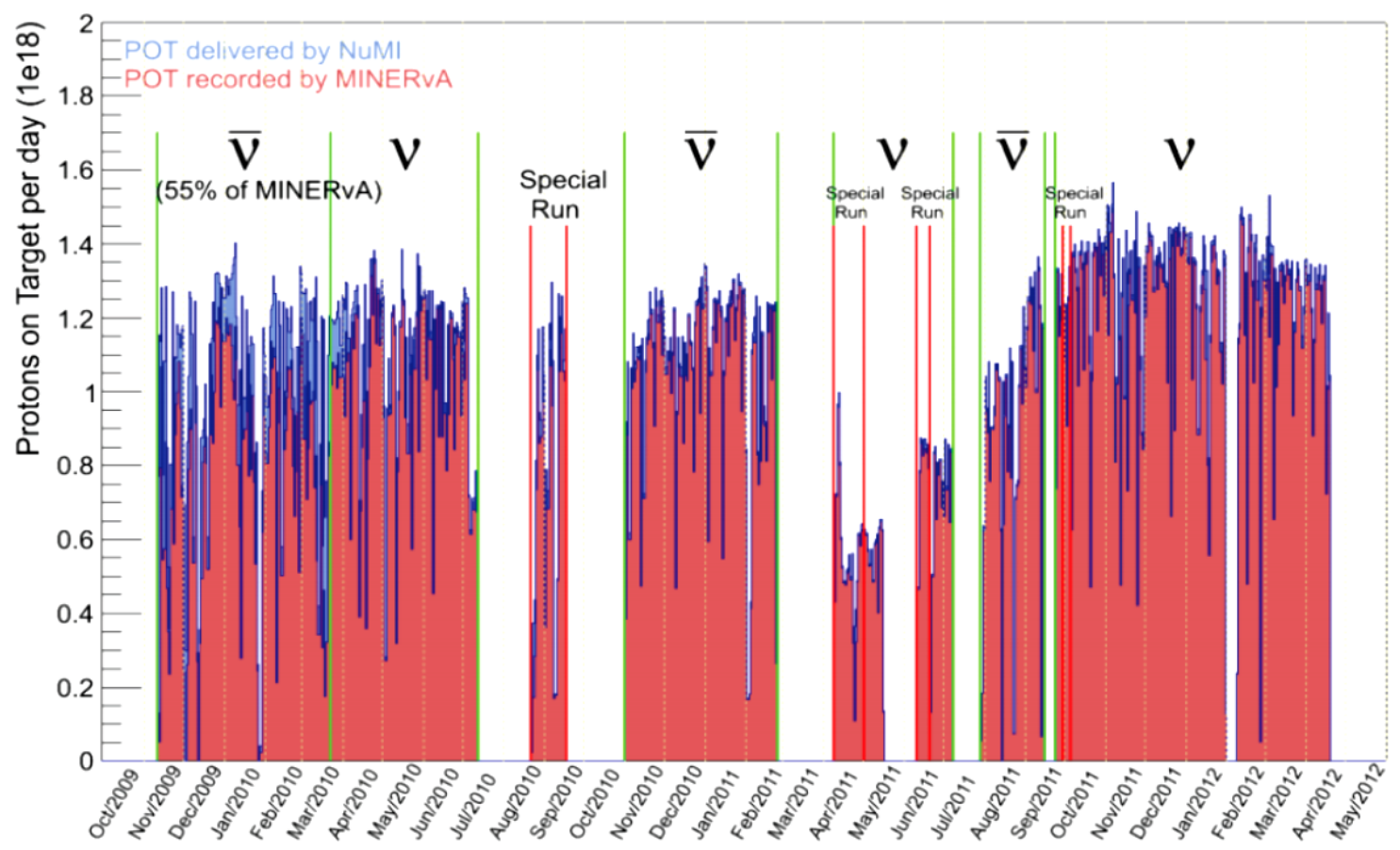

Figure 3.13. The MINER $\nu$ A total collected data for the Low Energy Run Period. The Special Runs correspond to the periods when the NuMI target-horn system was in a non-standard configuration.

The LE data presented in the analysis corresponds to four intervals of the NuMI $\nu_{\mu}$ focusing mode, where the Main Injector delivered $\sim 35 \times 10^{12}$ protons on target per beam spill to NuMI. The recorded protons on target (P.O.T) for each $\nu_{\mu}$-mode intervals are of the following,

1. data period of March 22, 2010 - July 12, 2010 with $0.941 \times 10^{20}$ POT

2. data period of May 7, 2011 - May 132011 with $0.025 \times 10^{20}$ POT

3. data period of June 22, 2011 - July 1, 2011 with $0.064 \times 10^{20}$ POT 
4. data period of October 18, 2011 - April 30, 2012 with $1.914 \times 10^{20}$ POT. 


\section{SECTION 4}

\section{CALIBRATIONS, SIMULTIONS, AND NEUTRINO EVENT RECONSTRUCTION}

The data provides information about the position, time, and energy deposition from the electronic signals (hits) collected by the MINER $\nu \mathrm{A}$ detector, where the raw signals are recorded by photomultiplier tubes, which decode the scintillation light produce by particles propagating throughout the detector. Figure 4.1 shows a schematic diagram of an optical readout channel, where each component is described in the previous chapter.

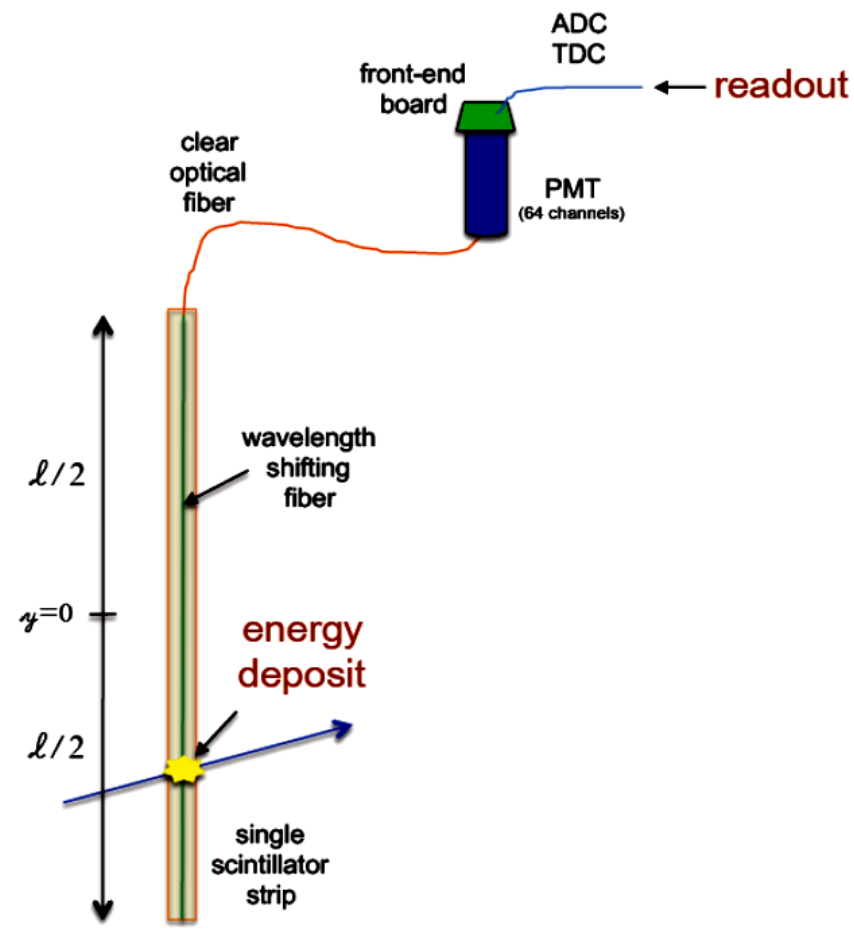

Figure 4.1. A schematic diagram of an optical readout channel. Reprinted from [51], Copyright (2013).

Figure 4.1 illustrates that the electronic signals are digitized by the Front-End Boards and read out as raw time-to-digital (TDC) counts and raw analog-to-digital (ADC) 
counts. Recall that the width of the NuMI beam spill (gate) is approximately 8-10 $\mu$ sec and the width of the FEB's gate is $\sim 150$ ns. Due to the high intensity neutrino flux, multiple neutrino interactions can be observed per NuMI beam spill in the MINER $\nu \mathrm{A}$ detector. The hit time calibration includes correcting for the transport time of the scintillation light from the scintillator hit position to the PMT and the time response of the electronics, as well as the grouping of hits that are within a similar time frame (time slice) of a detector gate that is triggered within the NuMI gate. Time slicing is discussed in subsection 4.3.1. In addition to the hit time calibration, the raw ADC counts must be convert into energy, which gives an estimation of the energy deposited in each scintillator strip. There are several effects (describe in the next subsections) that must be taken into account in order to convert the raw ADC counts into units of energy. The conversion from ADC counts, $A D C_{i}$, to the energy deposited, $\mathrm{E}_{i}$ per scintillator strip $i$ is computed as,

$$
E_{i}=\left[C(t) \cdot S_{i}(t) \cdot \eta_{i}^{a t t} \cdot e^{l_{i} / \lambda_{\text {clear }}} \cdot G_{i}(t) \cdot Q_{i}(A D C)\right] \times A D C_{i}
$$

Here,

- $\mathrm{C}(\mathrm{t})$ is the absolute energy scale constant as a function of time, which converts the measured photoelectrons into units of energy in $\mathrm{MeV}$,

- $\mathrm{S}_{i}(\mathrm{t})$ is the relative channel to channel energy scale constant as a function of time, which corrects for the energy variations of the scintillator strips,

- $\eta_{i}^{a t t}$ is a scintillator strip attenuation correction factor, which encodes differences in strip-to-strip response,

- $\mathrm{e}^{l_{i} / \lambda_{\text {clear }}}$ is the exponential correction factor for a clear optical fiber of length, $l_{i}$ with an attenuation constant of $\lambda_{\text {clear }}=7.83 \mathrm{~m}$,

- $\mathrm{G}_{i}(\mathrm{t})$ is the PMT gain per channel as a function of time,

- $\mathrm{Q}_{i}(\mathrm{ADC})$ is the conversion from an analog signal given by the PMT to the number of photoelectrons (charge),

where the energy calibrations that are responsible for the measured values of these terms are summarized in Section 4.1. 
Through a series of low and high level calibration techniques and pattern recognition reconstruction chains, the hits are converted into algorithmic objects that describe particles propagating throughout the detector. Within the MINER $\nu \mathrm{A}$ software framework, there exist sophisticated analysis routines which are designed to reconstruct neutrino interaction events out of these software objects. In addition, all of the various apparatus components of the MINER $\nu \mathrm{A}$ experiment and the neutrino interactions are simulated with Monte Carlo codes in order to understand the detector response and the measured data. This chapter provides an overview of the detector calibrations, the simulation of events observed in the MINER $\nu \mathrm{A}$ detector, and the reconstruction of both the calibrated data and simulated events into neutrino interactions.

\subsection{CALIBRATION}

The MINER $\nu \mathrm{A}$ experiment has two sets of calibrations, the Ex situ and In situ. The Ex situ calibrations were measured from various components of the detector before deployment into the fully assembled detector. The In situ calibrations were measured from the fully installed and instrumented detector. The following subsections, 4.1.1 and 4.1.2, give an overview of the calibration chains, Additional information is provided in Reference [51].

\subsubsection{Ex situ Calibration}

\section{The Module Mapper}

Before each module was installed in the detector, the module was placed in a custom designed module mapper as shown in Figure 4.2. The module mapper scanned the entire surface of a module from above using two ${ }^{137} \mathrm{Cs}$ radioactive $\gamma$-sources that were mounted in motion-driven source carriages. The main purpose of the module 
mapper was to measure the optical attenuation response of the WLS fibers per scintillator strip as a function of the position along each strip. The mapper also served as the final quality assurance check for identifying dead channels and anomalous strip response distributions that may correspond to broken fibers.

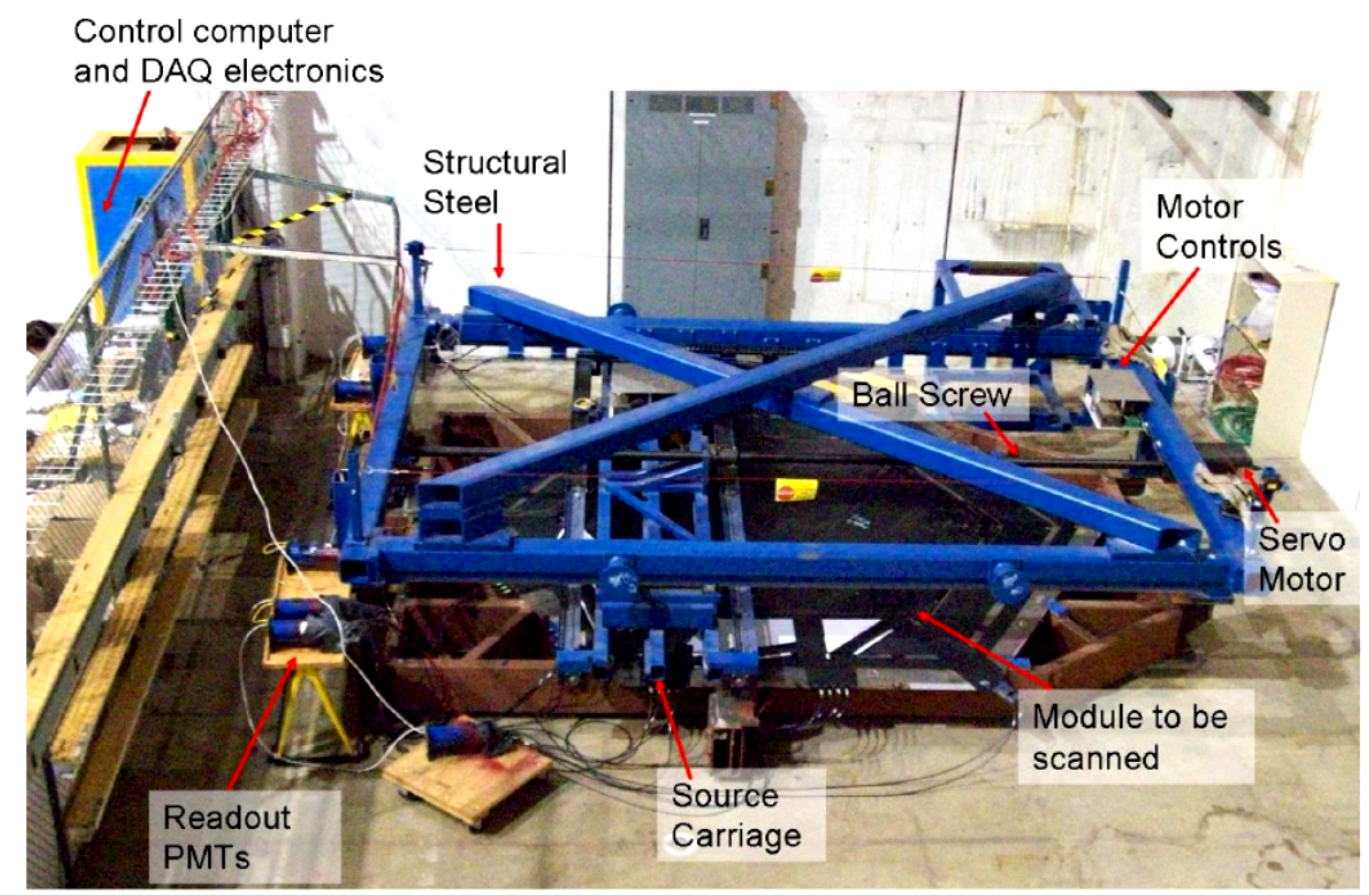

Figure 4.2. A picture of the Module Mapper. Reprinted from [51], Copyright (2013).

\section{The Photomultiplier Tubes Test Stand}

Before being installed in the cylindrical steel box, each PMT is subjected to quality assurance tests on a PMT test stand as shown in Figure 4.3. The PMT is tested for the following: linearity, efficiency, dark noise, channel-to-channel amplification variation, and optical cross-talk. After the PMT passes the first set of quality assurance requirements, it is prepared for deployment into the optical box. Once all of the apparatus parts are assembled and enclosed in the PMT box, the entire unit undergoes additional quality assurance checks. These checks ensure that the unit operates sufficiently and the components are properly aligned. Any mis-alignments between 


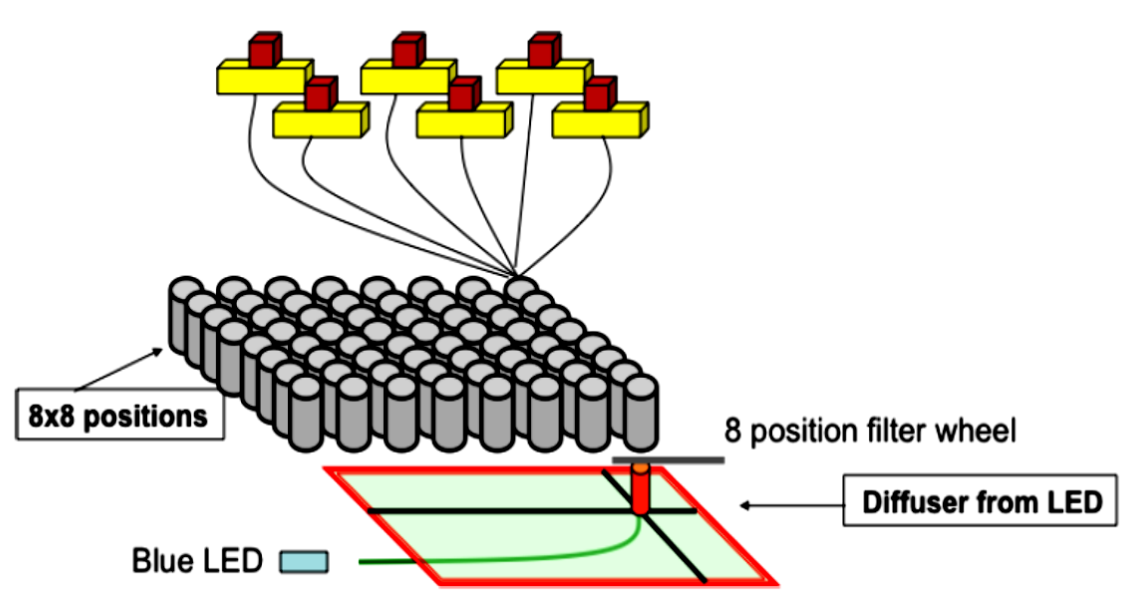

Figure 4.3. A schematic diagram of the PMT test stand. Six PMTs, which are represented by the red cubes, are mounted onto fiber cookies which are represented by the yellow rectangular boxes. A bundle of six clear optical fibers is connected to six PMTs, such that one channel of each PMT can be read out using the same clear optical cable. Light from a blue LED is absorbed by a green WLS fiber and illuminates one pixel in each of the six PMTs, simultaneously. Reference [51], Copyright (2013).

the PMT and the fiber cookie will most likely result in additional optical cross-talk between adjacent pixels which was not observed in the original quality assurance test.

\section{The Front-End Boards Response}

Each FEB is subjected to a series of quality assurance checks, such as high voltage control, discriminator and electronic cross-talk measurements. In addition, the charge calibration is extracted from each of the 507 FEBs prior to installation. 10 $\mathrm{pF}$ capacitors, which are supplied with various known high voltages, are used to inject external charges into the FEB, where the response is characterized by a fit to a tri-linear function. The fitted function describes the high, medium, and low gain responses for each electronic channel. The conversion from the raw ADC counts to a linearized charge is extracted from the parameters obtained from the tri-linear fit. 


\subsubsection{In situ Calibration}

Equation 4.0.1 shows that for majority of the terms, which are needed to convert the raw ADC counts to units of energy, are time-dependent. Thus, many calibrations are performed at regular intervals from the fully-instrumented detector. Most of the in situ calibrations are performed using a selection of rock muons, which are muons that are produced from the neutrino charged current interactions in the rock upstream of the detector. At least one rock muon event is likely to be observed during each NuMI gate. In addition to the high event rate, rock muons are minimum ionizing particles (MIP) which either exit or range out in the detector, thus making them simple to identify topologically. As a result, rock muons serve as an excellent source for calibrating the detector. However, the pedestal and gain calibrations are performed with data that is recorded which does not use the beam activity trigger. These special calibration runs are usually taken at the beginning of a run sequence or during a NuMI beam downtime, when the Main Injector is not delivering protons to NuMI. The in situ calibrations are described in the following subsections, which include the pedestal subtraction, PMT Gain, scintillator plane alignment, absolute energy scale, and timing calibration.

\section{Pedestals Subtraction}

Even in the absence of an input charge, channels can measured low level electronic signals called pedestals. A pedestal signal in a digital channel corresponds to the zero of the input signal. The pedestals varied from channel to channel. The pedestal for each channel must be subtracted off in order to accurately measured the amount of energy deposited in each scintillator strip. The pedestals are determined when there are not any external induced charge activities being applied to the MINER $\nu \mathrm{A}$ detector. The pedestal data is taken once every 32 subruns, where a subrun consists of approximately $750 \mathrm{NuMI}$ gates. The reason for collecting pedestal data so frequently, 
is simply because pedestals can drift over time. Figure 4.4 shows an example of a high gain pedestal distribution for a single channel, where the calibration is performed by A. McGowan of the MINER $\nu$ A Collaboration.

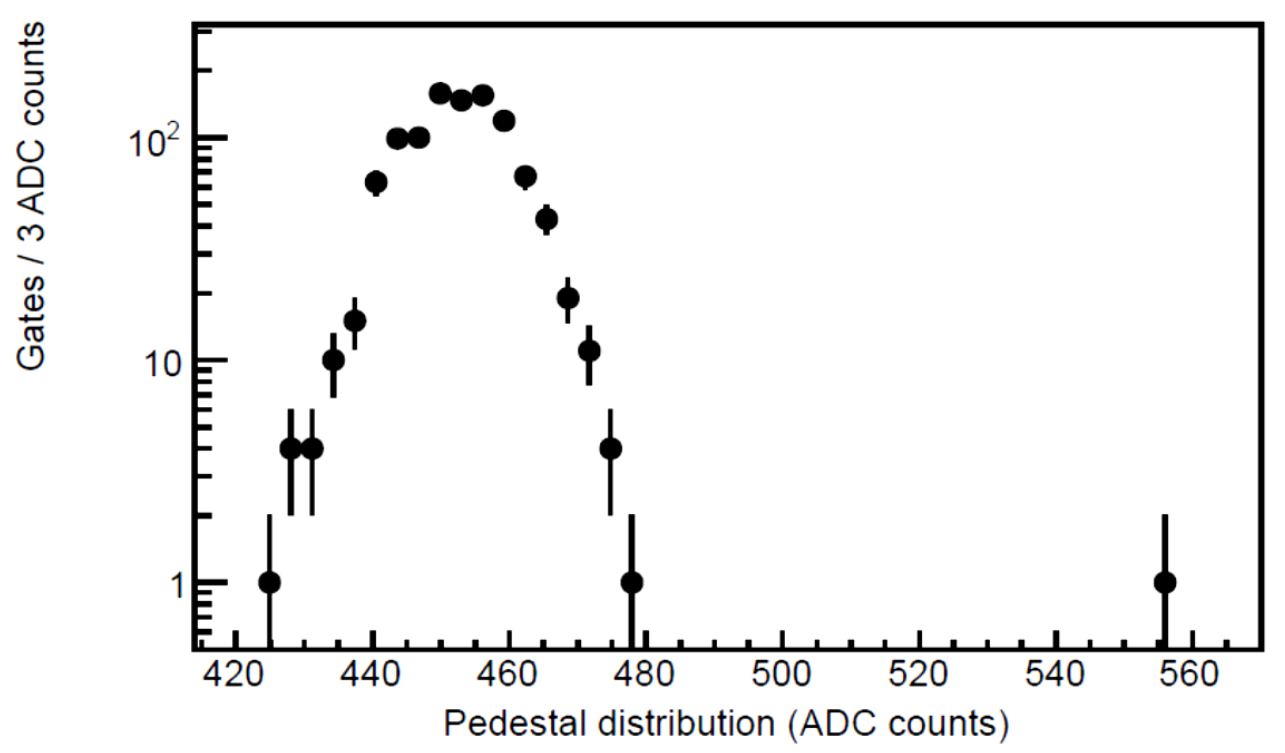

Figure 4.4. Plotted is an example of a high gain pedestal distribution for a single channel, where the outlier may originated from a cosmic ray event. Reprinted from Reference [51], Copyright (2013)

\section{PMT Gains}

The gains for the PMTs can change over time as shown in Figure 4.5, where the calibration is performed by B. Eberly of the MINER $\nu$ A Collaboration. For monitoring the PMT gain, a light injection (LI) system is utilized. Each PMT optical box has two ODU ports which connect to clear optical fiber cables from the LI system. A polypropylene diffuser is embedded in each ODU port and spreads the light across the front face of the PMT. A blue AlGaInP LED from the LI system can generate a signal on the order of a few photoelectrons (PEs), where the LI system has been optimized to pulse at a $1 \mathrm{PE}$ level for the gain calibration. For a PMT with $n$ dynodes, the gain is calculated using a probabilistic computation which models the gain amplification 
linearly with the number of PEs that are emitted from the PMT photocathode. The gain at each dynode stage can be written as

$$
g=\frac{\sigma_{Q}^{2}-\sigma_{p}^{2}}{\bar{Q}\left(1+w^{2}(g)\right) e}
$$

where $\sigma_{Q}^{2}$ is the variance of a probability function that is function of the charge, $\sigma_{p}$ is the width of the pedestal, $\bar{Q}$ is the mean of the pixel's pedestal-subtracted anode charge distribution, $e$ is the magnitude of the electron charge, and $w^{2}$ is defined as,

$$
w^{2} \equiv \sum_{j=1}^{n}\left(\prod_{i=1}^{n} \frac{1}{g_{i}}\right)
$$

Here, $\mathrm{g}_{i}$ is the gain per pixel and is proportion to the voltage, $\mathrm{V}_{i}^{\alpha}$, at each dynode stage and the value used for $\alpha=0.75$, where this value leads to a systematic uncertainty of less than $\% 1$ on the gain calibration.

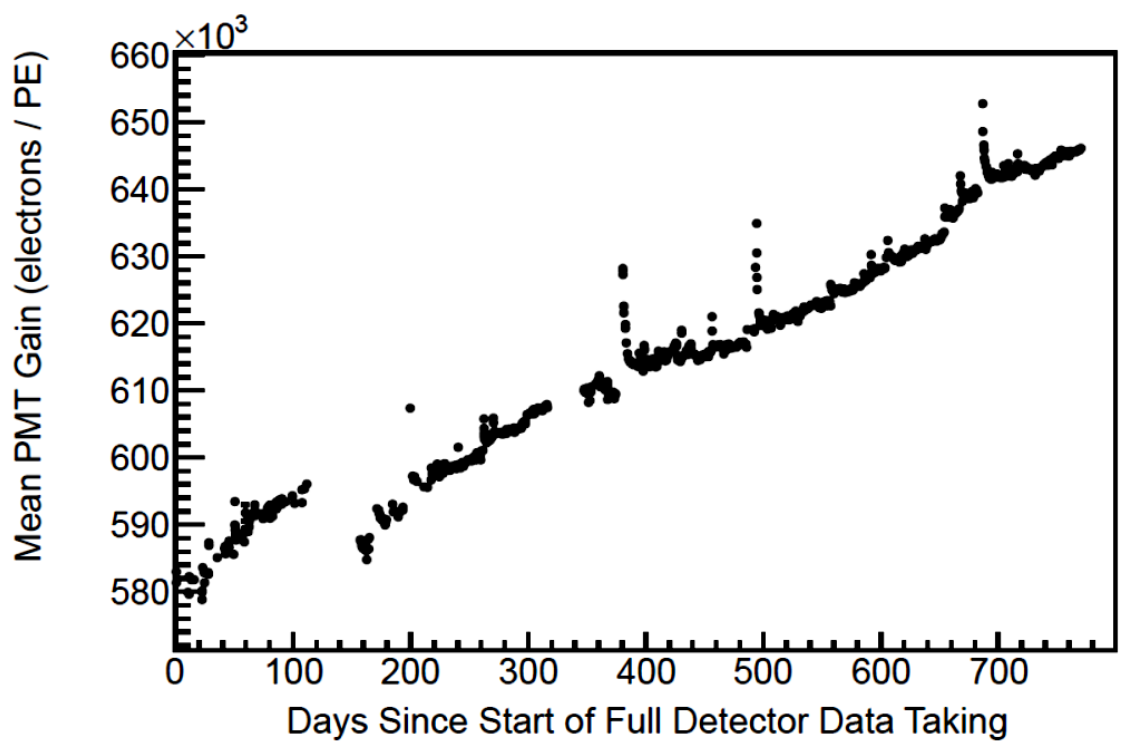

Figure 4.5. The plot shows the average PMT gain as a function of time. The dip at day 120 is a result of adjusting the high voltages. Reprinted from Reference [51], Copyright (2013). 


\section{Scintillator Plane Alignment}

Recall that the MINER $\nu \mathrm{A}$ modules are mounted in OD steel frames which are placed in a steel rack that is mounted in the Earth and parallel along the direction of the NuMI beam. During the installation and instrumentation of the detector modules, the planes relative position can be slightly rotated along the longitudinal axis. Also, due to gravity, the planes in the different types of modules have various stress and strain tolerances within a frame. These position variations are measured using a plane-based alignment procedure, which uses rock muons that enter the front of MINER $\nu \mathrm{A}$ and exit the back of the detector (commonly referred to as throughgoing rock muons). As shown in Figure 4.6, a muon rock traverses a plane and can deposited energy into two adjacent strips, where the total plane length is from the point when the rock muon intersects the front base position of the triangle strip to the back base position.

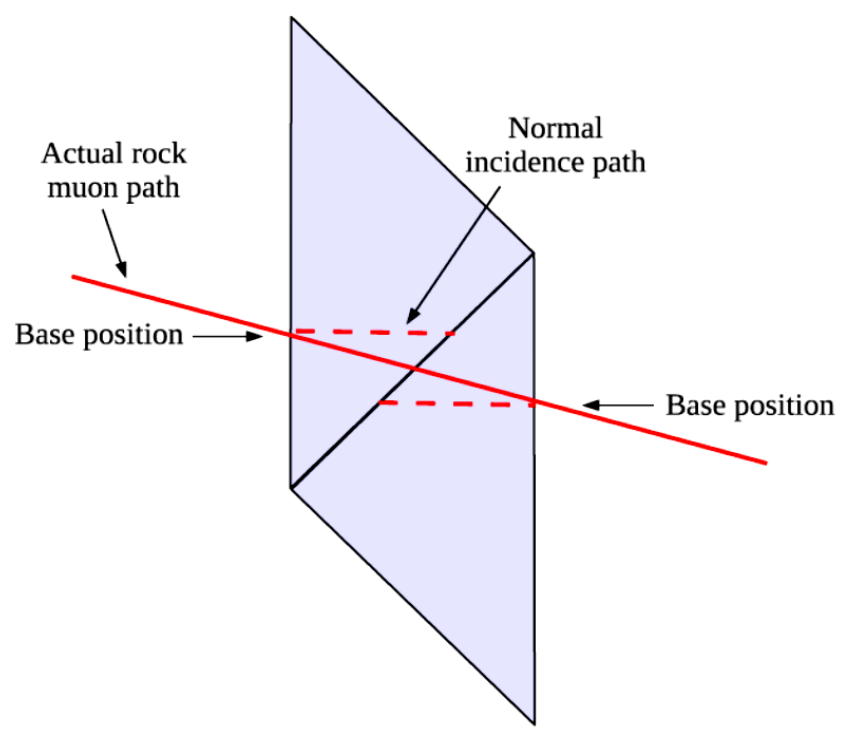

Figure 4.6. A schematic diagram of a rock muon traversing two adjacent strips. Rock muons that travel throughout the detector usually deposited energy in one or two strips per plane. Reprinted from Reference [66], with courtesy from C. Marshall.

The majority of the rock muons selected for this calibration travel along the direction of the beam. Thus, for this selected group of rock muons, the triangular strip 
maximum path length is from the center of the triangular base to its peak. With this information, the measured energy loss is corrected to the normal incidence such that the distribution peaks at the center of the strip as indicated in Figure 4.7. The zero position is the expected value of the center position for a triangular strip relative to its position within the plane.
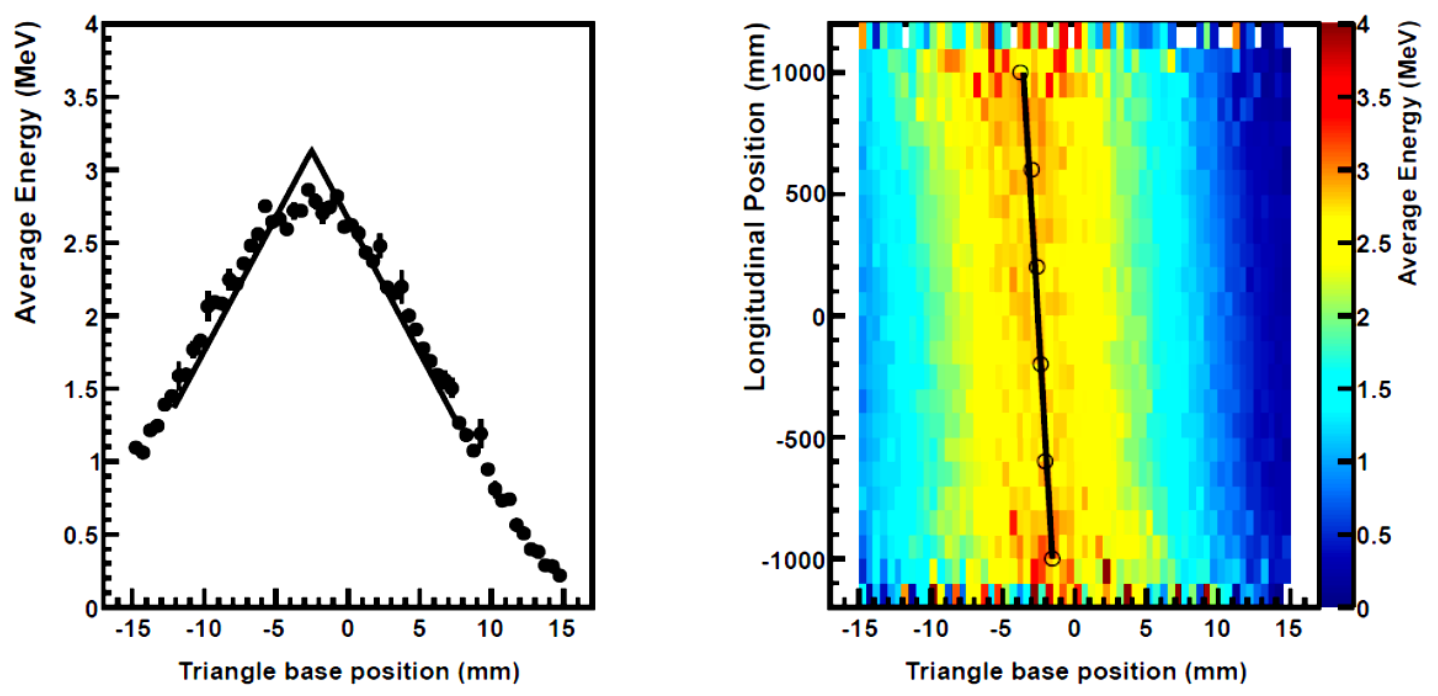

Figure 4.7. The plots show the scintillator plane alignment measurements as described in the text. Reprinted from Reference [51], Copyright (2013).

As shown in the left plot of Figure 4.7, the offset between the measured peak and nominal value is defined as the shift parameter. The shift parameter is extracted for all of the scintillator strips. Since the planes can be slightly rotated around the $Z$ axis with the assembly, the shift parameter is plotted as a function of the longitudinal position as indicated in the right plot of Figure 4.7 and the slope from the linear fit gives a determination of the rotation angle. This procedure ensures that the planes are efficiently aligned, where the calibration is performed by C. Marshall of the MINER $\nu \mathrm{A}$ Collaboration. 


\section{Scintillator Strip's Channel to Strip's Channel Energy Variations}

There are several reasons why the scintillation light levels may fluctuate between the strips. For example, this can be the result of the differences in the mixture of batches of scintillator material or air bubbles in the optical epoxy used to couple the WLS fiber in a strip. To remove these variations, a channel-to-channel (commonly referred to as strip-to-strip) calibration is applied which uses multiplicative constants to normalize each strip's observed energy deposition relative to the others. This calibration utilizes through-going rock muons and is an iterative procedure which

involves extracting the constants using the truncated mean of the observed $\frac{d E}{d x}$ per strip of the rock muons as shown in Figure 4.8. The Figure 4.8 shows the truncated mean $d E / d x$ of the sample of rock muons for all channels in the ID before and after applying the multiplicative constants to the measured $d E / d x$ of the rock muons. This technique is able to identify dead channels as well as fibers that are software mapped incorrectly to the electronic channels. The strip-to-strip response variation is performed after the plane-based alignment calibration and by C. Marshall of the MINER $\nu \mathrm{A}$ Collaboration.

\section{Absolute Energy Scale}

The absolute energy scale calibration uses through-going rock muons and is performed after the strip-to-strip calibration. Unlike the channel-to-channel calibration, the absolute energy scale accounts for the light level response of particles propagating throughout the detector. MINER $\nu$ A uses GEANT Monte Carlo codes to simulate particles traversing the detector. GEANT4 modeling of the energy loss by muons in the scintillator material is well understood, where muons lose energy by ionization [12]. Therefore, the absolute energy scale calibration extracts a muon equivalent unit (MEU) factor by tuning the simulated $d E / d x$ to the measured $d E / d x$. The extracted MEU factor is utilized to convert the observed photoelectrons into units of energy. 


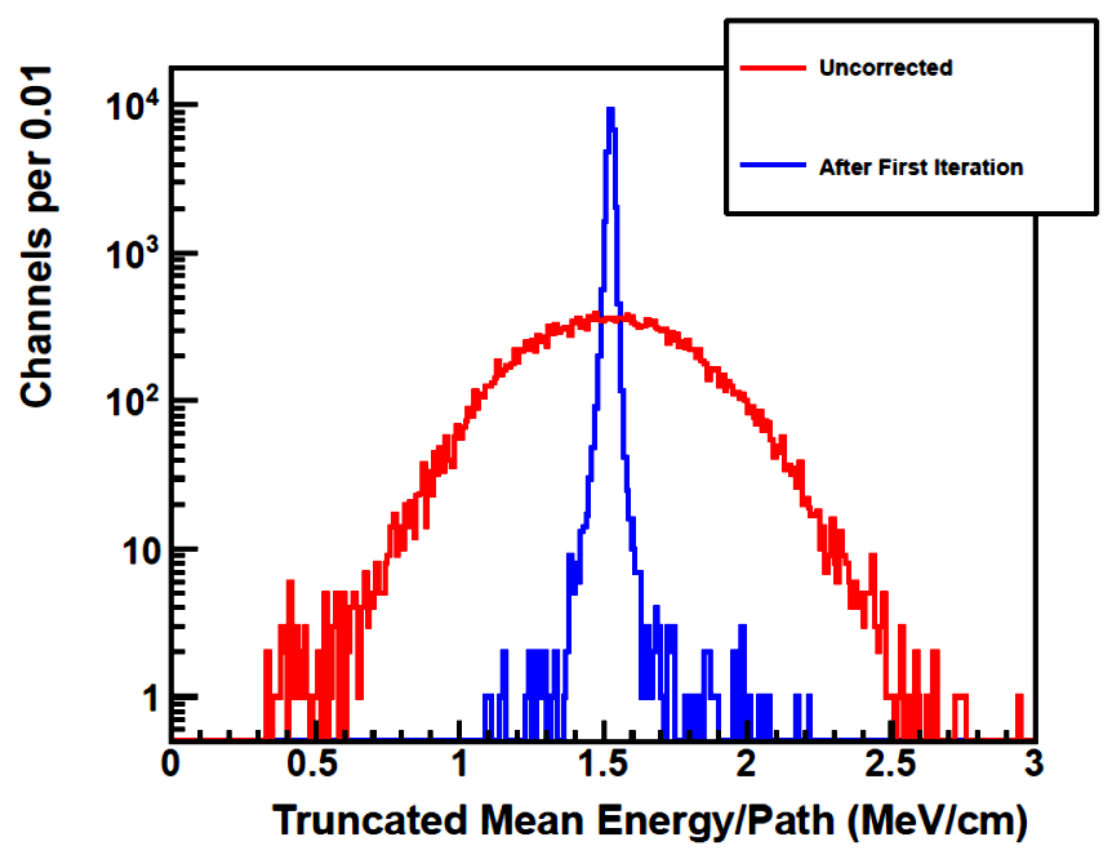

Figure 4.8. Results for all ID channels of the truncated mean energy loss before and after applying one iteration of the strip-to-strip response calibration. The uncalibrated data (red histogram) has a width of $\sigma=19.1 \%$ and after the first iteration the distribution (blue histogram) decreases to a width of $\sigma=1.6 \%$. Not shown on the plot, but the procedure is preformed in two iterations. Reprinted from Reference [66], with permissions from C. Marshall.

The scintillator was found to suffer from aging effects, which reduce the light yield over time, and in addition, the overall scintillator response is affected by the temperature of the detector hall. To account for the time variation of the light levels, the technique selects the rock muon events from data in two-day intervals and extracts a MEU factor per interval. Figure 4.9 shows a plot of the comparisons between simulated and real data reconstructed for cluster energy of rock muon events. The absolute energy scale calibration is performed by A. Mislivec of the MINER $\nu \mathrm{A}$ Collaboration. An overview of the clustering of energy is illustrated in the subsection 4.3.1 

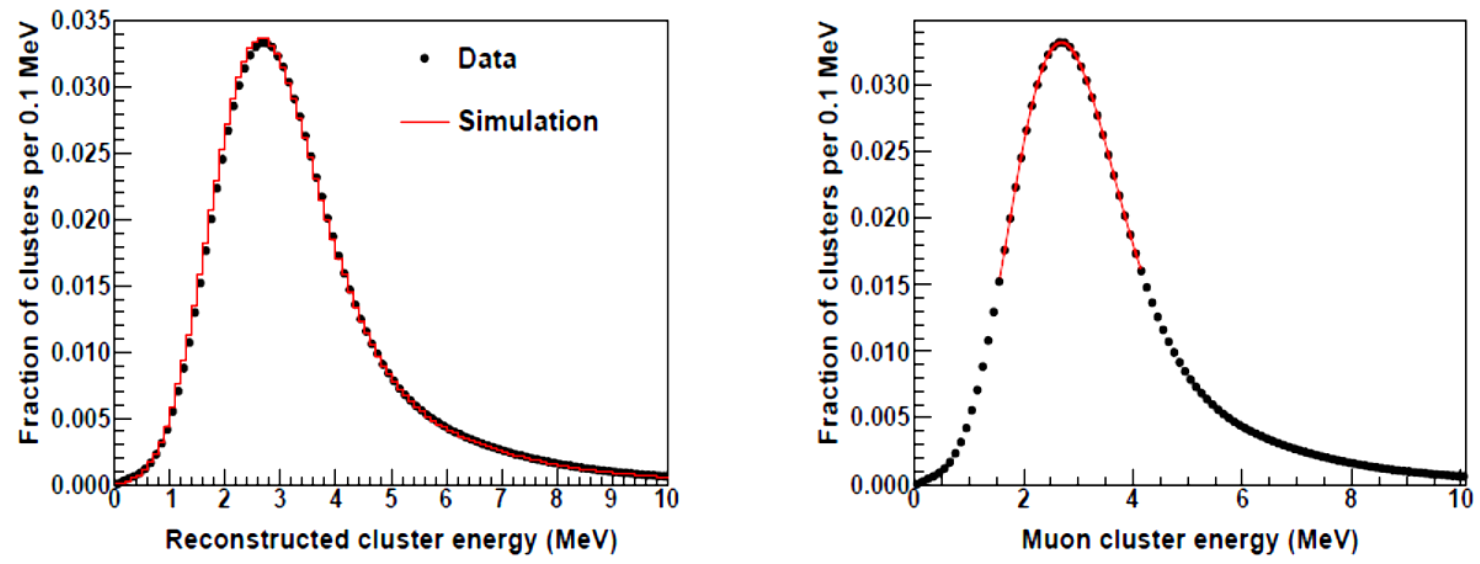

Figure 4.9. The energy loss distributions of clusters on rock muon tracks. On the left is the simulated and data distributions and the right shows a fit to the peak of the data distribution. The left plot shows that the comparison between the simulated and measured energy loss of rock muon events in the detector is remarkable good. Reprinted from Reference [51], Copyright (2013).

\section{Timing}

The timing calibration also utilizes the hit time and number of PEs from rock muons. The purpose is to correct for the timing variations observed in the MINER $\nu \mathrm{A}$ detector. These variations can included the light transport time in the optical fiber, channel-to-channel offsets, and time slewing. Recall that the FEBs are daisy-chained, thus the channel-to-channel time offsets consist of the decay time in the cables and the time offset between the FEB chains, where the time offsets between the FEB channels are negligible. The time slewing is primarily caused by the time it takes the signal to surpass the discriminator threshold. Time slewing is energy dependent, because signals with large counts of PEs pass the discriminator threshold much earlier than the signals with a smaller PEs as indicated in Figure 4.10. 


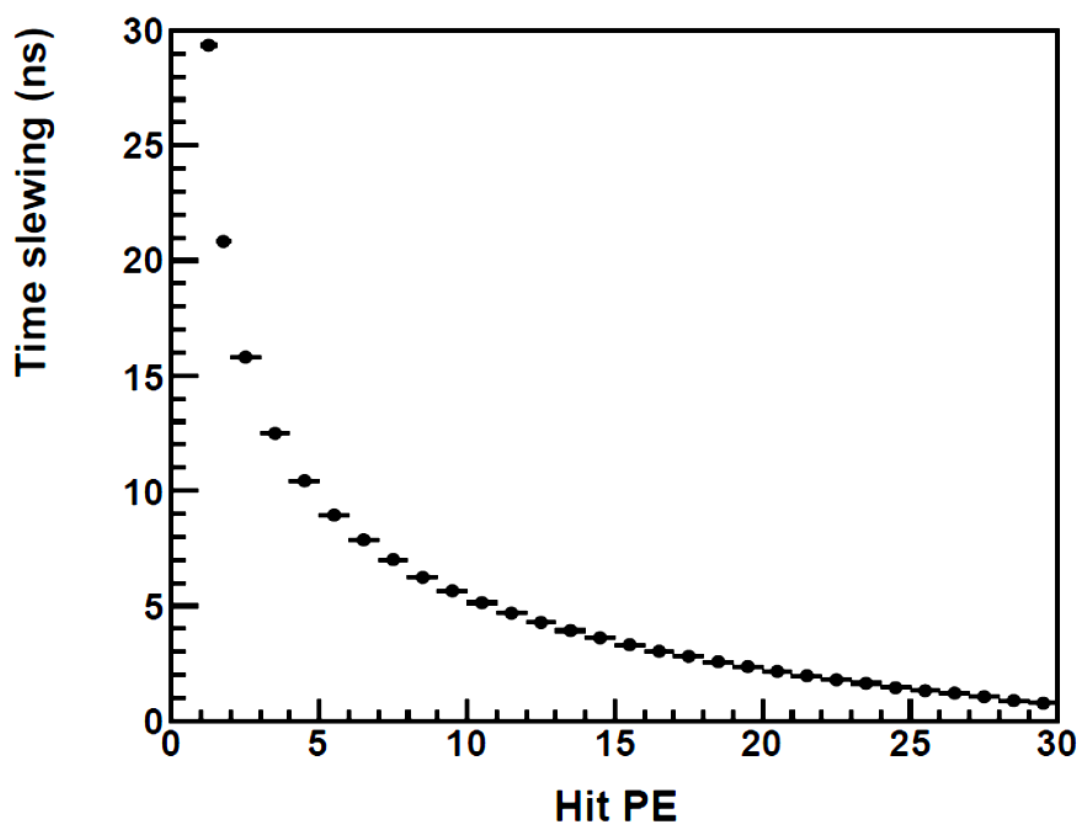

Figure 4.10. The measured timing slew as a function of photoelectrons for through-going rock muons, as describe in the text. Reprinted from Reference [51], Copyright (2013).

The speed of light in the fiber along with its length are used to correct the hit time for the light transport time along the optical fiber path. The time slewing is corrected by fitting the measured energy-dependent time slewing curve (see Figure 4.10) with a third order polynominal. The technique requires that the energy-dependent time slewing is fitted for each group of 32 channels which are read out by the same highgain TriP-t chip. The timing calibration is an iterative procedure, which searches for the best timing calibration constants through the optizimation of the hit time residual. The hit time residual (relative time) is the difference between the calibrated time of each hit and the truncated-mean time of hits along the rock muon tracks. The timing calibration is performed by A. Mislivec of the MINER $\nu$ A Collaboration. 


\subsection{SIMULATION}

The MINER $\nu \mathrm{A}$ experiment uses various simulation (Monte Carlo codes) packages for modeling the NuMI beamline, MINER $\nu$ A detector, the MINOS detector, and the neutrino interactions. First, the neutrino flux, which is produced by the NuMI beamline, is predicted based on the G4numi (Geant4 version 9.2.p03 NuMI) beam Monte Carlo (MC). The neutrino flux is consumed by GENIE (Generates Events for Neutrino Interaction Experiments version 2.6.2), which generates neutrino interactions and transports the recoil hadrons through the nucleus. The particles are propagated through the detector using Geant4 (version 9.2.p03), along with additional MINER $\nu \mathrm{A}$ simulation codes which more accurately describe the detector and electronic responses of the particles. Finally, the particles that exit MINER $\nu \mathrm{A}$ are propagated to MINOS, where Geant3 version 21.14a is utilized for the MINOS simulation. This section gives an overview of the simulation of the NuMI flux and the MINER $\nu \mathrm{A}$ detector. The analysis presented in this dissertation does not rely on the MINOS detector, therefore the MINOS simulation will not be discussed any further.

\subsubsection{NuMI FLUX}

The NuMI beamline, and the hadrons which are produced from the proton beam collisions with the NuMI production graphite target (Carbon), are simulated using the G4numi package. G4numi is a Geant4-based implementation of the NuMI beamline and by default uses the QGSP (Quark Gluon String Pre-compond) hadronic physics for modeling the hadron production, reinteractions with the production target and magnetic horns, and propagation [67]. In spring of 2013, MINER $\nu$ A switched from using the QGSP to FTFP_BERT (Fritiof with Pre-compound and Bertini cascade) as the input hadronic physics model in G4numi. Figure 4.11 shows that the neutrino energy spectrum depends significantly on the input hadronic model. The discrepan- 
cies demonstrate that there is uncertain on the modeling of the mesons propagation in the production target and magnetic horns [68].

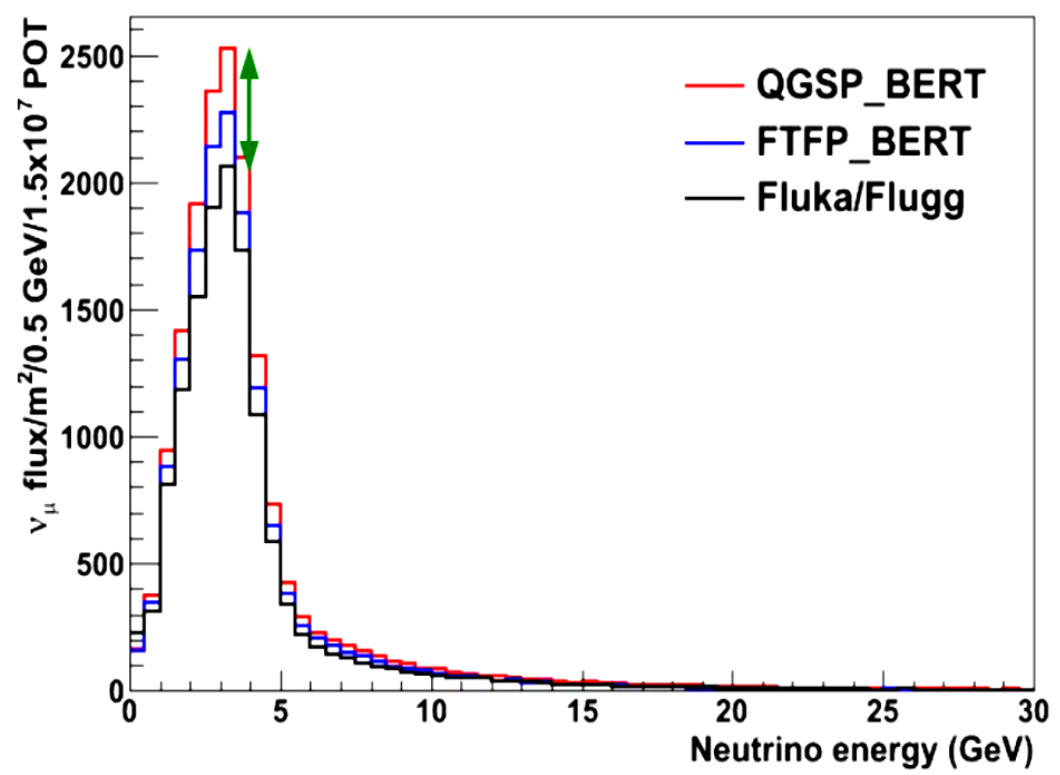

Figure 4.11. Plotted is various neutrino energy spectrums using the same MC geometry, but different input hadronic physics models. The green arrow illustrates the discrepancy between the peaks of the distributions. Plot courtesy from M. Kordosky of the MINER $\nu \mathrm{A}$ Collaboration.

Currently, MINER $\nu \mathrm{A}$ has several active programs in progress exploring different datasets and techniques to tune the neutrino flux. These include the following:

- using data collected from the muon monitors by measuring the muon flux that is produced from the mesons and relating it to the neutrino flux [69],

- exploring special runs data, data collected where the NuMI target-horn system is positioned in a non-standard configuration [68],

- analyzing the low $\nu$ method, meaning events with low energy transfer to the nucleon for a neutrino interaction process [70],

- applying the neutrino-electron scattering as a constraint by sampling various regions of the predicted neutrino flux [71],

- re-weighting the model prediction of hadrons produce by the $p C$ collisions with external hadron production data.

Only the external hadron production data has been utilized for constraining the flux which is used in the analysis presented in this dissertation. The raw external 
hadron data has better agreement overall with the FTFP_BERT hadron model for the prediction of both the $\bar{\nu}_{\mu}$ and $\nu_{\mu}$ energy spectrums, which explains why MINER $\nu \mathrm{A}$ decided to switch from QGSP to FTFP_BERT for G4numi hadronic physics model $[72]$.

\section{Hadron Production Reweighting}

The predicted neutrino flux is corrected by reweighting the G4numi events based on the measurements from external $p C$ hadron production data. The collected datasets which are used to reweight the production cross sections for the $p C \rightarrow \pi^{ \pm} X$, $p C \rightarrow K^{ \pm} X$, and $p C \rightarrow p^{ \pm} X$ collisions are from the following experiments:

- NA49 experiment (Large Acceptance Hadron Detector for an Investigation of Pb-induced Reactions at the CERN SPS) collected data using a $158 \mathrm{GeV} / \mathrm{c}$ proton beam [73],

- Barton, et. al produced measurements for the $p C$ collisions with a $100 \mathrm{GeV} / \mathrm{c}$ beam using the Fermilab Single Arm Spectrometer in the M6E beamline [74].

Recall that the NuMI production target is exposed to a $120 \mathrm{GeV} / \mathrm{c}$ proton beam, which differs from both of the external hadron production experiments. To account for the energy differences, an energy scaling correction is applied only to the NA49 dataset using the Monte Carlo package FLUKA [72]. Both NA49 and Barton have reported the measured cross-sections as functions of the transverse momentum, $p_{T}$, and Feynman x, $x_{F}$ variables, where

$$
\mathrm{x}_{F} \sim \frac{2 p_{L}}{\sqrt{s}}
$$

with $\mathrm{p}_{L}$ the longitudinal momentum and $\sqrt{s}$ the total center of mass energy. The $p C \rightarrow \pi^{ \pm} X$ cross-section comparisons between the FTFP_BERT predictions and NA49 measurements are shown in Figure 4.12, where the focusing peak region for the NuMI $\nu$-beam (the left plot in Figure 4.12) is $\mathrm{x}_{F}=[0.0,0.16]$ and $\mathrm{p}_{T}=[0.0,0.6]$ $\mathrm{GeV} / \mathrm{c}$.

The reweighting factor for the NA49 data is written as, 

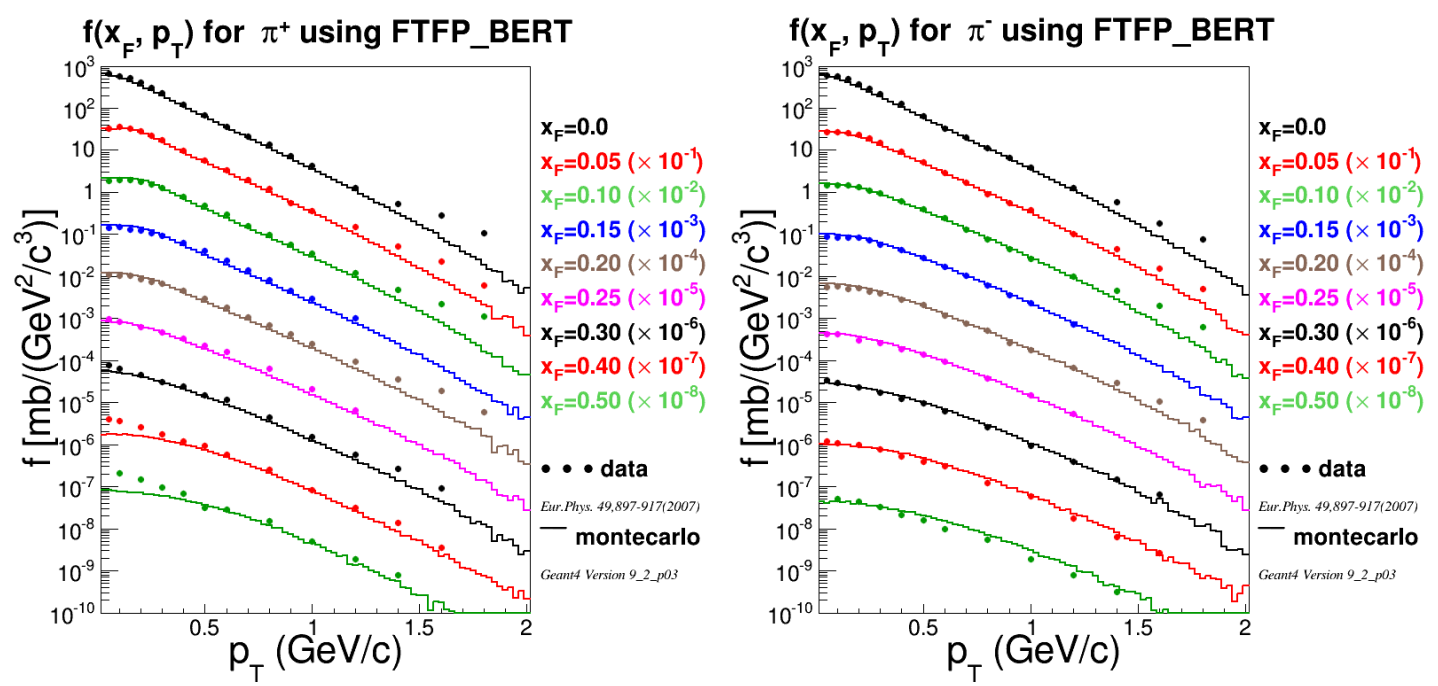

Figure 4.12. The left (right) plot shows a comparison of the FTFP_BERT predicted cross section to the NA49 data for the $p C \rightarrow \pi^{+} X\left(p C \rightarrow \pi^{-} X\right)$ collisions. Plots courtesy of L. Aliaga of the MINER $\nu$ A Collaboration.

$$
w_{N A 49}=\frac{\sigma_{N A 49}\left(x_{F}, p_{T}, E=158 G e V\right)}{\sigma_{\text {model }}\left(x_{F}, p_{T}, E\right)} \cdot \frac{\sigma_{F L U K A}\left(x_{F}, p_{T}, E\right)}{\sigma_{F L U K A}\left(x_{F}, p_{T}, E=158 G e V\right)},
$$

where the second term is the energy scaling correction which was mentioned above. The re-weighting factor for the Barton dataset is,

$$
w_{\text {Barton }}=\frac{\sigma_{\text {Barton }}\left(x_{F}, p_{T}, E=100 G e V\right)}{\sigma_{\text {model }}\left(x_{F}, p_{T}, E\right)},
$$

where an energy scaling correction is not applied. The extracted weights from the NA49 and Barton datasets are applied to specific kinematic regimes and the scattered hadrons from the $p C$ interactions and are summarized in the table 4.2.1.

The events which fall outside of these kinematic ranges (as indicated in table 4.2.1) are assigned a re-weighting factor equal to one. In addition, weights are applied to correct for the attenuation of the primary proton beam that interacts within the 
Table 4.1. A summary of the kinematic regimes for re-weighting the hadron production.

\begin{tabular}{lcc}
\hline \hline$p C$ Interaction Type & Kinematics & Dataset \\
\hline$p C \rightarrow \pi^{ \pm} X$ & $\mathrm{x} F<0.5$ & NA49 \\
$p C \rightarrow \pi^{ \pm} X$ & $\mathrm{x} F>0.5$ & Barton \\
$p C \rightarrow K^{ \pm} X$ & $\mathrm{x} F<0.2$ & NA49 \\
$p C \rightarrow p X$ & $\mathrm{x} F<0.95$ & Barton \\
\hline \hline
\end{tabular}

target. Recall that the NuMI production target is approximately 2 nuclear interaction lengths.

\section{The Flux Results}

Figure 4.13 shows the results for the predicted neutrino flux for both the NuMI $\nu_{\mu^{-}}$ beam and $\bar{\nu}_{\mu}$-beam, where the NuMI $\nu_{\mu}$-beam flux is ulitized for the analysis presented in this thesis. The distributions only show the spectrums up to $20 \mathrm{GeV}$, while this analysis produces a cross section measurement using the neutrino flux integrated from 0 to $100 \mathrm{GeV}$.
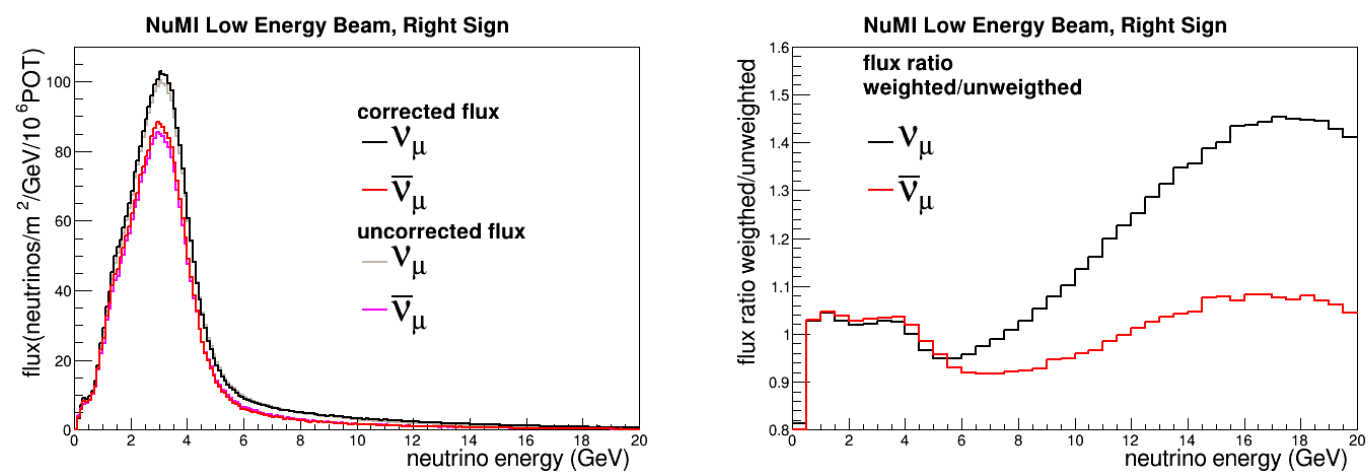

Figure 4.13. (Left) $\nu_{\mu}$ and $\bar{\nu}_{\mu}$ simulated fluxes with and without the hadron production weights applied. (Right) Ratio of the hadron re-weighting distributions, where the dip region corresponds to the falling edge of the focusing peak. Plots are a courtesy of L. Aliaga of the MINER $\nu$ A Collaboration.

The method that is used to extract an uncertainty on the flux will be discussed later in the dissertation. 


\subsubsection{NEUTRINO EVENT GENERATOR}

MINER $\nu \mathrm{A}$ uses the ROOT-based Monte Carlo generator, GENIE for generating neutrino interactions in nuclear matter, as well as modeling the transportation of the hadrons through the nucleus [75]. GENIE is capable of simulating neutrino interactions with an energy spectrum ranging from $\sim 1 \mathrm{MeV}$ to $\sim 100 \mathrm{TeV}$ for all neutrino species interacting with a broad variety of nuclear targets. One of the challenges for GENIE is to incorporate the theoretical predictions and phemomenological models of neutrino interactions in the few-GeV (neutrino energy ranging from 1 to $5 \mathrm{GeV}$ ) regime, which is essential for the current and near-future neutrino oscillation experiments, as well as for evolving the interpretation of the nuclear physics observed from the neutrino scattering data. This section summarizes the GENIE models implementations that are used for simulating the neutrino interaction channels that are relevant to the analysis presented in this thesis, as well as the hadron transportation models.

\section{Modeling the Nuclear Medium}

GENIE uses the formalism of the Relativistic Fermi Gas (RFG) model for describing all neutrino processes. The impluse approximation (see section 2.3) is used for modeling the neutrino-nucleon scattering, where the recoil nucleons are assigned an average binding energy based on the electron scattering data. Recall that nucleons within a short range relative to each other can interact strongly as described in subsection 2.3.1. GENIE has an implementation of the Bodek and Ritchie model that describes the high momentum tail of the nucleons after the nucleon-nucleon interaction. However, all neutrino processes are modeled assuming scattering from a quasi-free nucleon. As mentioned eariler, GENIE also applies Pauli blocking, which requires that the recoil nucleon from the elastic and quasi-elastic processes is outside of the Fermi sea $\left(\mathrm{p}_{N}>\mathrm{p}_{F}\right)$. Table 4.2 lists GENIE parameters for the Fermi momentum and binding energy for the carbon, iron, and lead nuclei. 
Table 4.2. The Relativistic Fermi Gas Model parameters for the Quasielastic Scattering.

\begin{tabular}{c||cc}
\hline Nucleus & $\mathrm{p}_{F}$ for neutron (proton) $(\mathrm{MeV} / \mathrm{c})$ & $\mathrm{E}_{b}(\mathrm{MeV})$ \\
\hline Carbon & $221(221)$ & 25 \\
Iron & $263(251)$ & 36 \\
Lead & $283(245)$ & 44 \\
\hline
\end{tabular}

\section{Cross Section Models}

GENIE provides the differential cross section on an event-by-event basis for an interaction channel for a given flavor of neutrino which can scatter on a wide variety of nuclei. There are several stages in the GENIE simulation to calculate the differential cross section. First, the total neutrino cross section, $\sigma_{t o t}\left(\mathrm{E}_{\nu}\right)=\sum_{i} \sigma_{i}\left(\mathrm{E}_{\nu}\right)$ is pre-calculated. The total cross section includes the variety of different scattering processes, such as scattering from the nucleus, individual nucleons, quarks, and atomic electrons. Next, GENIE determines if the neutrino interacts. If the neutrino undergoes a scattering process, the physical process, $\alpha$, is sampled via the probability function, $P_{\alpha}\left(E_{\nu}\right)=\sigma_{\alpha}\left(E_{\nu}\right) / \sigma_{t o t}\left(E_{\nu}\right)[76]$. Once the interaction channel is known, the modeling of the differential cross section for that particular process determines the event kinematics. The interaction channels that are relevant to this analysis are quasielastic, charged current resonance production, and charged current deep inelastic scattering (DIS).

\section{Quasielastic Scattering}

As mentioned in subsection 2.2.1, the quasielastic scattering is modeled using the Llewellyn-Smith formalism [21]. The vector form factors are related to electromagnetic nucleon form factors by the conserved vector current and are given by the BBBA2005 parameterization (see subsection 2.2.2). Recall that the nucleon form factors, $\mathrm{G}_{E}^{p}\left(Q^{2}\right), \mathrm{G}_{E}^{n}\left(Q^{2}\right), \mathrm{G}_{M}^{p}\left(Q^{2}\right)$, and $\mathrm{G}_{M}^{n}\left(Q^{2}\right)$, are extracted from both the Rosenbluth measurements and polarization transfer measurements. A fit to the polarization transfer data is done using the Kelly Parameterization [77], where the fit is given by 


$$
G\left(Q^{2}\right)=\frac{\sum_{k=0}^{n} a_{k} \tau^{k}}{1+\sum_{k=1}^{n+2} b_{k} \tau^{k}},
$$

where $\mathrm{G}\left(\mathrm{Q}^{2}\right)$ (also known as the Kelly Form Factors) are the following,

- $\mathrm{G}_{E}^{p}\left(Q^{2}\right) / \mathrm{G}_{D}\left(Q^{2}\right)$,

- $\mathrm{G}_{E}^{n}\left(Q^{2}\right) / \mathrm{G}_{D}\left(Q^{2}\right)$,

- $\mathrm{G}_{M}^{p}\left(Q^{2}\right) / \mu_{p} \mathrm{G}_{D}\left(Q^{2}\right)$,

- $\mathrm{G}_{M}^{n}\left(Q^{2}\right) / \mu_{n} \mathrm{G}_{D}\left(Q^{2}\right)$,

and $\tau=\frac{Q^{2}}{4 M^{2}}$ and the LaGrange polynomial $\mathrm{a}_{k}$ is initialized as $\mathrm{a}_{0}=1$ for the proton form factors and neutron magnetic form factor and $\mathrm{a}_{0}=0$ for the neutron electric form factor. In addition, the fit employs constraints at high $\mathrm{Q}^{2}$ based on the local quark-hadron duality [77]. As $\mathrm{Q}^{2} \rightarrow 0$, the BBBA2005 fits and Kelly Form Factors approach unity and is interpreted by the dipole approximation, with the exception of the neutron electric form factor which approaches zero. In addition, this also means that $\mathrm{G}_{E}^{p}\left(Q^{2} \rightarrow 0\right)=e, \mathrm{G}_{M}^{p}\left(Q^{2} \rightarrow 0\right)=\mu_{p}$, and $\mathrm{G}_{M}^{n}\left(Q^{2} \rightarrow 0\right)=\mu_{n}$.

Finally, the pseudoscalar form factor is related to the axial form factor as described in subsections 2.2.3, 2.2.4, and 2.2.5. In GENIE, the axial form factor is assumed to follow the dipole form and the free mass parameter, $\mathrm{M}_{A}$ is assigned a value of $0.99 \mathrm{GeV} / \mathrm{c}^{2}$.

\section{Resonance Production}

GENIE uses the Rein-Sehgal formalism for modeling the differential cross sections for the production of the baryon resonances, where the 16 resonances are summed incoherently to produce the total cross section [78]. These include the following channels,

$$
\begin{aligned}
& \mathrm{P}_{33}(1232), \mathrm{S}_{11}(1535), \mathrm{D}_{13}(1520), \mathrm{S}_{11}(1650), \\
& \mathrm{D}_{13}(1700), \mathrm{D}_{15}(1675), \mathrm{S}_{31}(1620), \mathrm{D}_{33}(1700),
\end{aligned}
$$




$$
\begin{aligned}
& P_{11}(1440), P_{13}(1720), F_{15}(1680), P_{31}(1910), \\
& P_{33}(1920), F_{35}(1905), F_{37}(1905), P_{11}(1671),
\end{aligned}
$$

where the resonances are labeled with the incoming partial wave $L_{2 I, 2 J}$ with $I$ is the isospin and $J$ is the total angular momentum. The resonance channel is the biggest source of background observed in the analysis presented in this dissertation. In addition, the $\Delta^{++} \rightarrow \pi^{+}+p$ reaction can contributed to the quasielastic-like signal, if the pion does not escaped the nucleus due to FSI. GENIE predicts that approximately $25 \%$ of the pions produced from the $\Delta^{++}(1232)$ resonance are absorbed in the nucleus. The hadron transportation in the nucleus is described below.

\section{Deep Inelastic Scattering}

The DIS cross section is modeled using the Quark Parton Model, where the low $\mathrm{Q}^{2}$ regime is described by modifications from Bodek and Yang. GENIE defines a DIS event as an event that does not produced an excited resonance from the neutrino inelastic scattering. DIS events that resemble resonance-like final states, meaning a nucleon and $1 \pi$ or $2 \pi$ in the hadron final state, are another source of background. After a final state interaction such that the pions are absorbed in the nucleus, the DIS event can resemble the quasielastic-like signal.

\section{Modeling Final State Interactions}

Hadrons produced from the neutrino scattering in nuclear targets with $\mathrm{A}>1$, may rescatter before escaping the nucleus. GENIE models the final state interactions using an intranuclear cascade simulation, which is handled by the INTRANUKE subpackage. The INC model assumes that the nucleus is an ensemble of quasi-free nucleons that contain Fermi motion and binding energy. The ejected hadron may interact with a single spectator nucleon through a series of encounters which is defined as a cascade. For the DIS process, before the quark interacts with the residual nucleus, the quarks are first modeled by the concept of hadron formation, the length and time 
it takes the quarks to materialize into hadrons. During the hadron formation, the strong interaction is turned off, thus the hadrons are more likely to exit the nucleus without experiencing any effects from FSIs.

The INC models the pions and nucleons propagation through the nucleus, with the primary goal that the missing energy lost in the nuclear medium is simulated correctly. The INC tracks particles in steps of $0.05 \mathrm{fm}$ through the nuclear environment. The probability that the hadron will interact at that step is based on the calculated mean free path which is a convolution of the hadron cross section, $\sigma_{h N}\left(E_{h}\right)$ and the density of the nuclear medium, $\rho(r)$. If the hadron interacts, then the interaction type is determined from the measured cross section for a particular process based on hadronnucleus scattering data[75]. The data which GENIE utilizes, comes from hadron interactions on Fe (see Figure 4.14), where the total reaction cross section for all other nuclei are obtained by scaling by $\mathrm{A}^{2 / 3}$. At the last stage, the kinematics are determined from the parameterization of data distributions or sophisticated nuclear models such as CEM03 model [79].
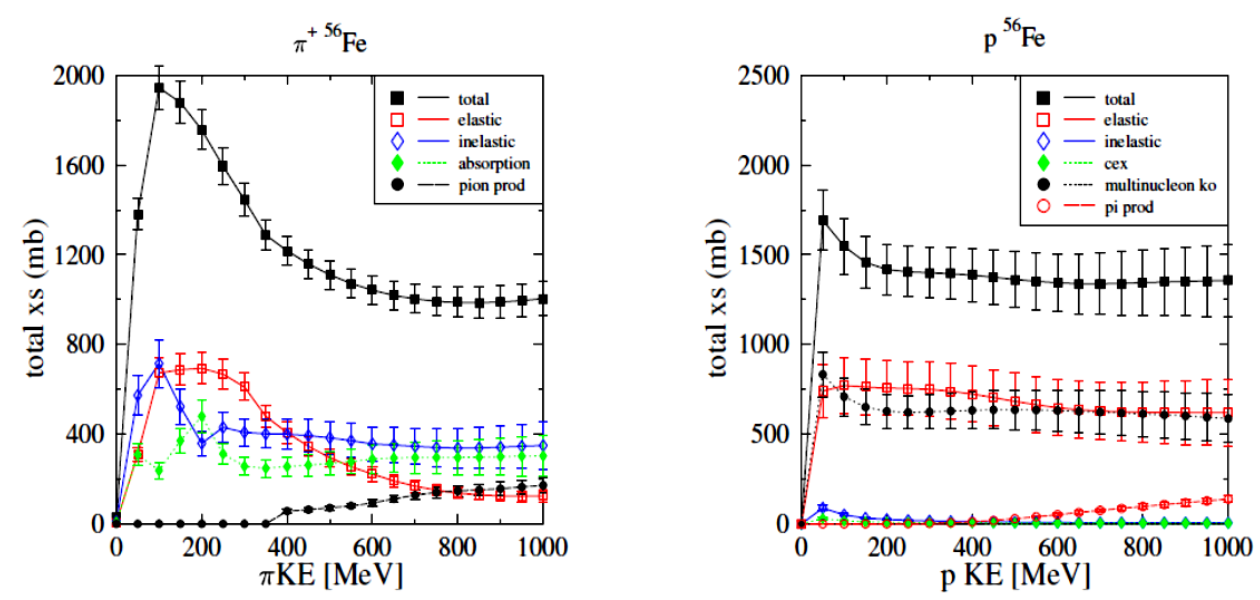

Figure 4.14. (Left) $\pi^{+} \mathrm{Fe}$ and (right) pFe cross sections which are utilized in the $h A$ INC model. The final state process is sampled from these data templates. Reprinted from Reference [79]. 
GENIE has two alternative hadron transportation models, denoted $h A$ and $h N$, where $h A$ is the default mode, which is used in the analysis presented in this dissertation. The $h A$ model implementation is described above and has been extensively tested and verified with data [79], [75]. The $h N$ model differs by walking each hadron through the nuclear environment and simulates the complete particle cascade using angular distributions as function energy which are obtained by the GWU group [79]. As a full INC model, the $h N$ calculates all of the reactions on all nuclei. Figure 4.15 shows an illustration which demonstrates the differences between the $h A$ and $h N$ models, where for the $h N$, the cascade is fully modeled.
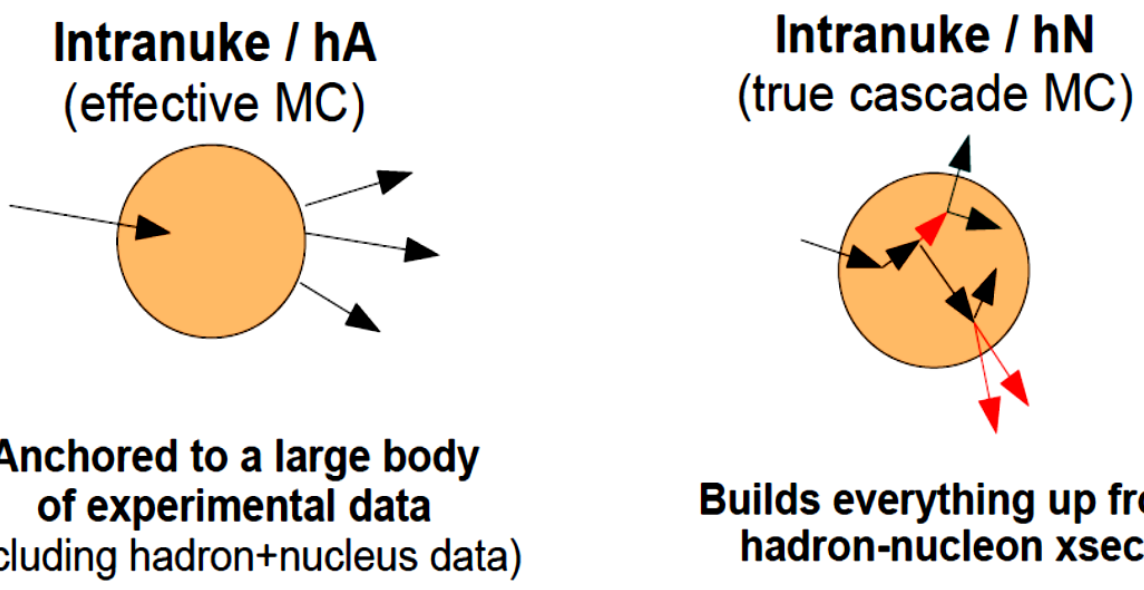

\section{Builds everything up from hadron-nucleon xsecs}

Figure 4.15. An illustration of the differences between the $h A$ and $h N$ intranuke cascade models, as mentioned in the text. Reprinted from Reference [80].

\subsubsection{SIMULATING EVENTS IN THE DETECTOR}

MINER $\nu \mathrm{A}$ uses Geant4 to simulate the propagation of the final state particles from the neutrino interactions through the detector [81]. Geant4 has an abundant collection of physics models which simulate particle interactions within nuclear matter for an overwhelming number of processes at a wide range of energies. Therefore, 
Geant4 is a useful Monte Carlo package for simulating the particles which satisfy the needs of the MINER $\nu \mathrm{A}$ experiement. The default Geant4 modules are used to simulate the behavior of particles traversing the detector, except for the hadronic physics module. The QGSP_BERT is the selected model, which includes the Bertini model for describing the hadron transportation in the nuclear environment at energies below $10 \mathrm{GeV}$.

In addition to neutrino interactions, other events such as rock muons and multiple neutrino interactions per time slice are observed in the collected data. To adequately model the complicated environment within a NuMI beam spill, the generated neutrino events are overlaid onto data gates. By overlaying data events on the simulated events, the simulation now includes events where the discriminator was not fired. Therefore, the overlaying procedure properly accounts for detector inefficiencies that are not modeled in the simulation, such as dead time and dead channels.

The MINER $\nu \mathrm{A}$ simulation framework also includes the simulation of the optical readout and electronics systems. This requires that Geant4 prediction of the particle's energy loss is converted into photoelectrons. The propagation of the simulated photoelectrons through a MINER $\nu \mathrm{A}$ simulated optical readout channel ensures that the simulated light output accurately describes the data. After this process, the tuned simulation (Monte Carlo) is now prepared to enter the reconstruction stage, where both the simulation and data have the same format and are reconstructed identical.

\subsection{RECONSTRUCTION}

Both the calibrated data and tuned Monte Carlo events are processed through a common set of reconstruction algorithms. This section gives an overview of the standard reconstruction passes for all events and the multi-track based specific reconstruction routines that give rise to the selection of the quasielastic-like events. Recall 
that these are events with one muon track and at least one proton track emerging from the neutrino interaction vertex.

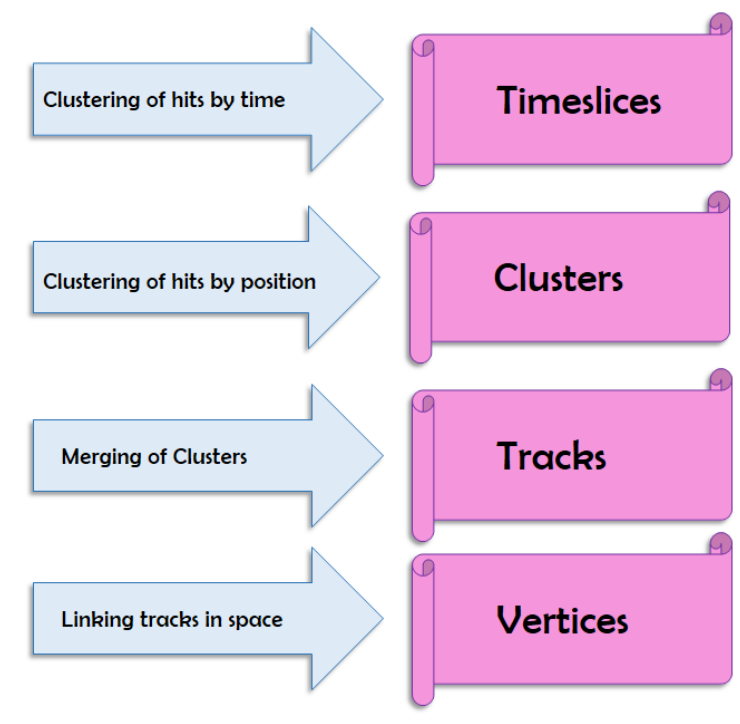

Figure 4.16. A flowchart of the reconstructed software objects in the MINER $\nu \mathrm{A}$ framework.

Figure 4.16 shows a flowchart of the algorithmic objects produce by the reconstruction, where the stages of the common reconstruction are the following:

1. Hits within a gate are bunched into smaller time windows called time slices, where the activity in the time slice usually represents a single event. Examples of an event can include a neutrino interaction, an incoming rock muon, and a Michel electron.

2. The hits per time slice are spatially grouped into objects called clusters. Clusters are formed in both the inner and outer detectors (ID and OD), separately.

3. Chains of pattern recognition algorithms form groups of clusters into 2-dimensional lines, which are eventually created into 3-dimensional objects called tracks. Currently, tracks are only reconstructed in the ID.

4. The vertex reconstruction links tracks to a common point in space. 
5. Tracks or hits (also commonly referred to as energy) in the MINOS detector are matched to tracks in the ID, where these topologies are automatically identified as muons. In addition, a separate matching routine associates energy in the OD to tracks in the ID, where this algorithm is executed before the MINOSmatching algorithm.

The standard reconstruction is designed specifically for an inclusive charged current neutrino interaction analysis, where the muon is captured by the MINOS detector and the un-tracked clusters are assumed to be apart of the hadronic recoil system.

\subsubsection{THE CLUSTERING OF HITS}

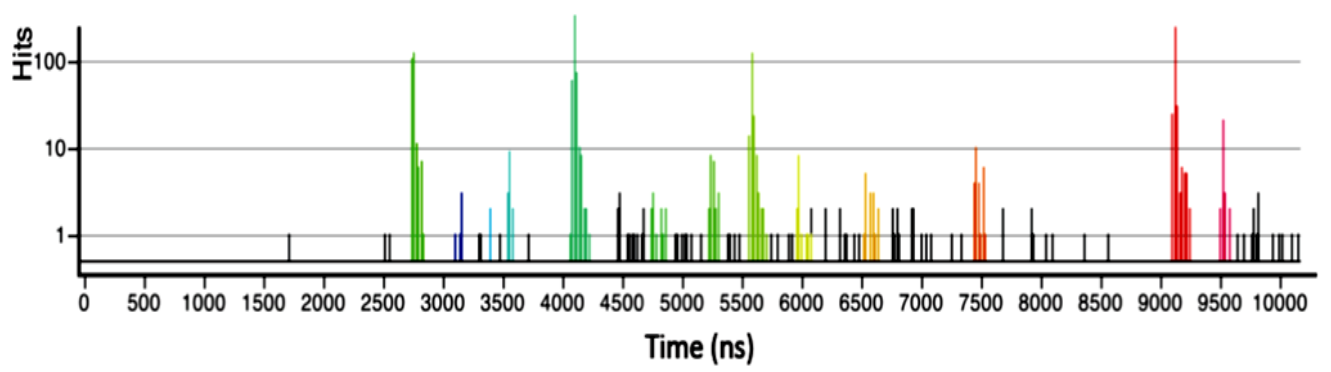

Figure 4.17. The time profile of the hits within a NuMI beam spill, where each colorbunch of hits corresponds to a different time slice. Reprinted from [51], Copyright(2013).

\section{Time slices Formation}

Multiple events can be observed during a single NuMI beam spill. Given that the MINER $\nu \mathrm{A}$ detector has a timing resolution of about $3.0 \mathrm{~ns}$, events within a gate can be efficiently untangled based on their timing profile. The time slices are formed by probing the NuMI gate time distribution, which consists of hits originating from both the ID and OD detectors. Probing forward in time, the photoelectrons within a time window of $30 \mathrm{~ns}$ are integrated. A time slice is created, if the integrated charge exceeds the default minimum value. Hits that occur close in time to the time slice are 
associated with that candidate. The adding of hits continue until the total integrated charge does not surpass the default minimum value. The width of the integration time for the time slices is given by the integration time of the FEBs, which is $\sim 150 \mathrm{~ns}$. Figure 4.17 shows the profiles of the time slices for a particular NuMI beam spill.

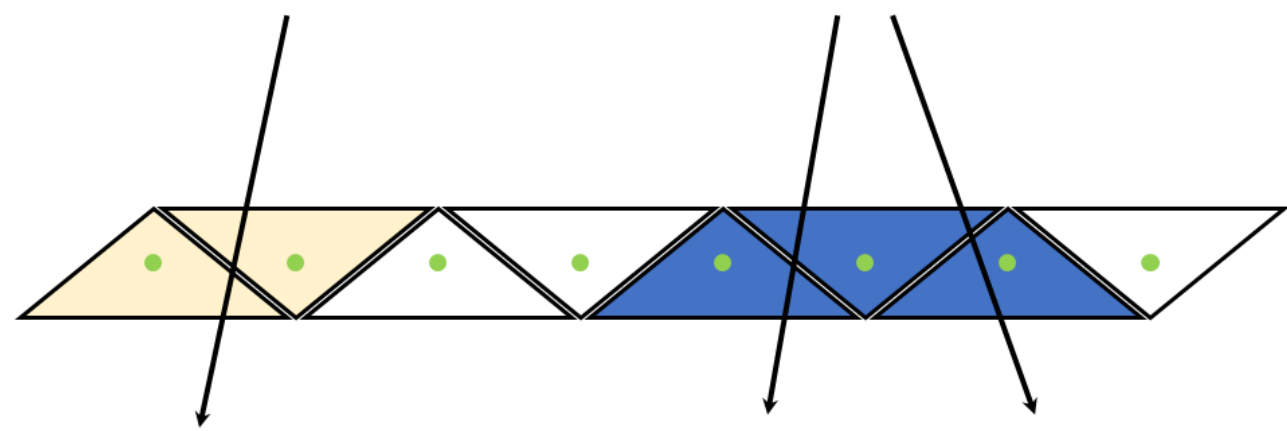

Figure 4.18. An illustration of the cluster formation in an ID plane. The arrows represent the trajectories of particles and the different colors correspond to the density of the energy deposition.

\section{Clusters Creation}

Hits in the ID are spatially grouped by plane and contiguous hits within a plane are merged to form clusters as indicated in Figure 4.18. In the OD, clusters maybe created out of adjacent hits in an OD bar, where an OD bar consists of only two rectangular strips as shown in Figure 4.19. All hits are promoted to clusters, which give rise to the one-hit cluster. Clusters are 2-dimensional objects with either a X-Z, $\mathrm{U}-\mathrm{Z}$, or $\mathrm{V}-\mathrm{Z}$ coordinate. For clusters in the ID, the $Z$ position is given by the $Z$ center position of the plane in the detector and the transverse position $(\mathrm{X}, \mathrm{U}$, or $\mathrm{V})$ is determined by the charge-weighted mean position of the hits. For clusters in the OD, the $Z$ position is taken from the $Z$-center position of the frame, and currently, the OD clusters are not assigned a transverse position. For all clusters, the energy is the sum of the energies of its hits and the time comes from its most energetic hit.

As shown in Figure 4.18 due to the alternating pattern of the triangular strips in an ID plane, a particle traversing the detector is most likely to deposit its energy into two adjacent strips. Since the energy loss profile of the particle depends on its mass 


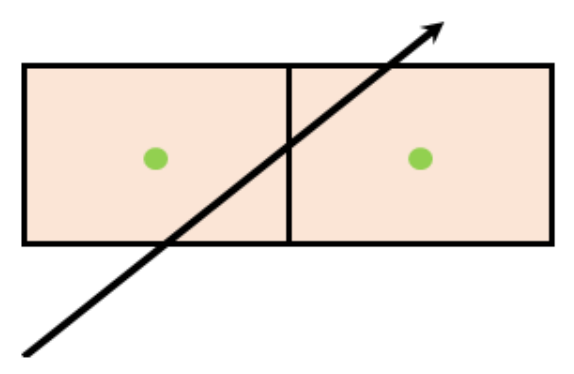

Figure 4.19. An illustration of the cluster formation in an OD bar. For many cases, the clusters are composed of an one-hit strip. This is mainly because most of the particles which enter and may exit the OD have large angles with respect to the beam axis.

and initial momentum, the energy density of the clusters can vary as the particle propagates through the detector. Thus, different particles with various momentum can generate various topologies of clusters. In addition, the production of various types of clusters depends on the event topology, as well. For example, a spray of high energy hadrons can produce very dense and wide clusters. Therefore, a cluster's energy, number of hits, energy of the individual hits, and the hit relative position to its neighboring hits are utilized for classifying clusters as one of the following: low activity, trackable, heavy ionzing, supercluster, or cross-talk. A description of each type of cluster is provided below.

- A cross-talk cluster is identified with hits which are correlated with the PMT pixels associated with a particular cluster. If hits are located directly adjacent to pixels which corresponds to the energy deposition of a particle, then those hits are tagged as cross talk.

- A low activity cluster contains a total visible energy, $\mathrm{E}_{t o t} \leq 1 \mathrm{MeV}$.

- A trackable cluster is composed of 1-4 hits, where its total energy ranges between 1-12 MeV. There must be at least one hit with $0.5 \leq \mathrm{E}_{\text {hit }}<12.0 \mathrm{MeV}$. If there are two hits such that each ranges from $0.5 \leq \mathrm{E}_{h i t}<12.0 \mathrm{MeV}$, the hits are required to be in adjacent strips. 
- A heavy ionizing cluster consists of 1-4 hits and has a total visible energy, $\mathrm{E}_{t o t} \geq 12.0 \mathrm{MeV}$. There must be 1-3 hits with $\mathrm{E}_{h i t}>0.5 \mathrm{MeV}$. If there are two or three hits with $\mathrm{E}_{\text {hit }}>0.5 \mathrm{MeV}$, the hits must be in sequential strips.

- A supercluster cluster does not satisfy any of the above conditions. These clusters are usually very wide with large energy deposition which correspond to multiple particles.

\subsubsection{TRACK RECONSTRUCTION}

A track is a reconstructed object which traces the trajectory of a particle. For particles which are not subjected to a dramatic change in direction due to an interaction in the detector, a single track is capable of estimating their trajectories. But for the case when the particle undergoes a hard collision, multiple linked tracks are needed for approximating its trajectory. Compared to the muons, the hadrons which propagate through the MINER $\nu \mathrm{A}$ detector are far more likely to require multiple tracks.

As mentioned previously, MINER $\nu \mathrm{A}$ common reconstruction framework has been developed particularly for the inclusive charged current channel, which implies that only the muon's trajectory is required to be tracked. In order to efficiently track the hadrons, multiple track algorithms must be utilized, these consists of the LongTracker and ShortTrackers. Since the LongTracker is designed and optimized to efficiently reconstruct good muon tracks, it is the only tracker activated during the running of the common reconstruction algorithms. Note that good means that the muon's trajectory has been successfully reconstructed. First, the formation of the trackbased events is discussed, followed by the various track pattern recognition schemes.

\section{Creating Events with Tracks}

The track-based event formation occurs at the common reconstruction phase and during the two track-based specific reconstruction. Although tracks are created at different processing stages, the track-based event formation is similar at both. There 
are several steps which are needed for creating high quality tracks. Figure 4.20 shows an illustration of the three steps that are described below.

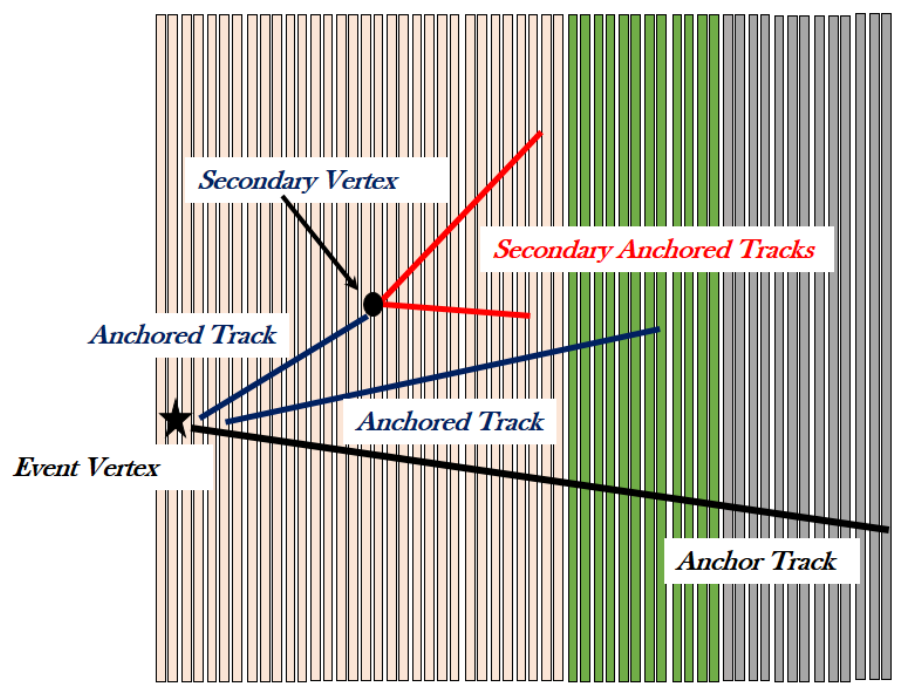

Figure 4.20. An illustration of the general procedure for creating a track-based event for a high multiplicity neutrino interaction in the MINER $\nu \mathrm{A}$ detector, see the text for the description of the labels.

1. Selecting the Anchor Track The anchor track is created at the common reconstruction stage using the LongTracker pattern recognition scheme. The LongTracker creates tracks out of the trackable and heavy ionizing clusters within a time slice. The longest track, which is typically the muon, is selected and identified as the anchor track, where all other tracks are removed, such that its clusters are freed to be consumed by the next round of track pattern recognition algorithms. The anchor track must spanned at least 25 planes in the detector, otherwise the track is discarded. The anchor track is assumed to be traveling in the forward direction. Thus, the event vertex is initialized at the most upstream cluster on the track. 
2. Creating Anchored Tracks Both the long and short trackers are used to create anchored tracks from clusters that are unused by the anchor track. The type of clusters which are selected depends on the track pattern recognition routine. In addition, each pattern recognition scheme has a different set of consistency requirements for the anchored tracks. The basic principal behind the consistency checks is to ensure that the vertices from the anchor and anchored tracks are compatible. If the anchored track is incompatible with the anchor track, then the anchored track is deleted. The search for anchored tracks continues until no further tracks satisfying the consistency criteria can be created. For each iteration, the anchor and anchored tracks are fitted simultaneously to form a common vertex (see subsection 4.3.3), where the updated event vertex replaces the previous one. Figure 4.20 demonstrates how anchored tracks are created.

3. Creating Secondary Tracks If the particle undergoes a hard collision, the trajectory can suddenly change direction or multiple particles can be produced. Therefore, the search for anchored tracks continues by leveraging the end position of each anchored track that was defined above. These secondary anchored tracks are commonly referred to as a kinked or forked tracks. If a secondary anchored track is created, then the anchored and secondary anchored track is fitted simulaneously to form a common vertex. This vertex is known as the secondary vertex, as indicated in Figure 4.20.

\section{The LongTracker}

The pattern recognition scheme for the LongTracker employs several stages, track formation, the adding of clusters to a track, and track cleaning. These various components are responsible for the robustness of the tracker. Figure 4.21 shows a plot of 
the track position resolution for through-going rock muons, where the plot includes all of the clusters along the muon track.

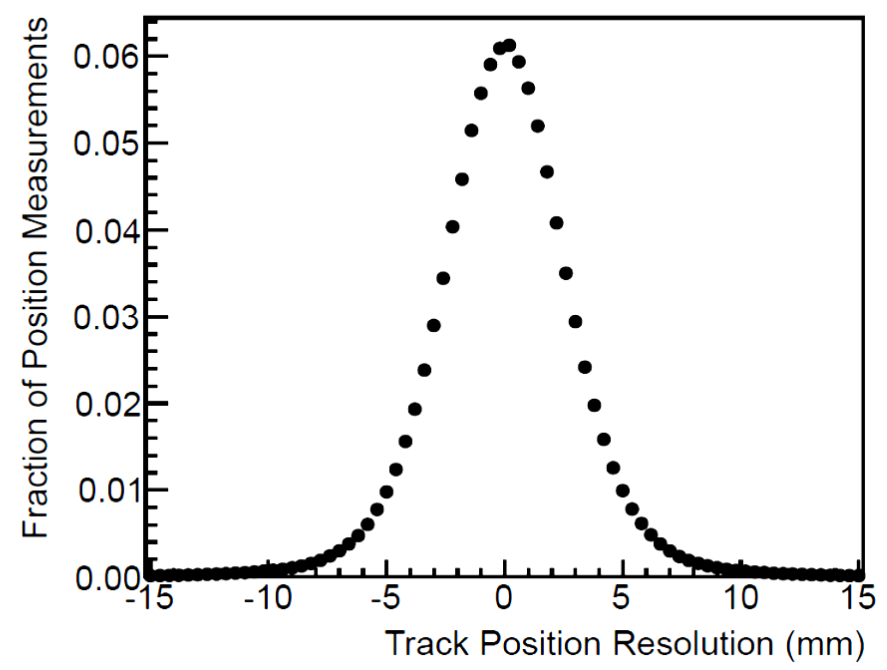

Figure 4.21. Difference between the fitted positions relative to the measured cluster positions for rock muon events. Reprinted from [51], Copyright(2013).

\section{Track Formation}

The first step in track formation is to form track seeds out of trackable and/or heavy ionizing clusters. The track seeds are grouped by views (X, U, or V module) and consists of three consecutive clusters in the same view. A single cluster is allowed to belong to multiple seeds. Each seed is fitted to a 2-dimensional line, where the $\chi^{2}$ from the least squares fit determines the quality of the seed. These enforced conditions prevent the reconstruction of tracks with angles greater than $60^{\circ}$ with respect to the longitudinal axis. Thus, the ShortTrackers are needed to reconstruct recoil hadrons with larger angles.

Track seeds are combined to form track candidates, where the seeds in the same view are merged if the following conditions are satisfied:

1. share at least one cluster,

2. can not share different clusters in the same plane, 
3. the slopes are consistent.

The merging procedure starts from the downstream end of the detector and stops toward the upstream region. This serves a way to avoid the influence of heavy vertex activity which may of have developed from a high energy neutrino interaction. After all of the possible track candidates are created, then the compatible candidates may joined, even in the presence of a missing cluster in a scintillator plane between two candidates.

The final merging step is executed sequentially by two different techniques (2dimensional and 3-dimensional algorithms) with the common goal to create 3-dimensional objects called tracks. The 3-dimensional routine searches for all possible combinations of $\mathrm{X}, \mathrm{U}$, and $\mathrm{V}$ orientations, where a track is created if the candidates overlap along the longitudinal axis and are compatible with the same line. After the 3-dimensional algorithm has tested all of the permitted track combinations and has exhausted its merging capabilities, the 2-dimensional routine is then executed. The 2-dimensional procedure searches and examines track candidates in pairs in order to create tracks. This method is quite sufficient for forming tracks when the track (observed by the eye) in a particular view is obscured by detector activity or inefficiencies such as missing clusters.

Due to the prerequisite of the track candidate combinations as mentioned above, the minimum number of planes a track can spanned is eleven and nine for the 3dimensional and 2-dimensional tracking algorithms, respectively. As a result, the ShortTrackers are essential for reconstructing particles which span less than nine planes and scatter at high angles with respect to the longitudinal axis. Finally, all created tracks are fitted by a Kalman filter, where the filter accounts for multiple Coulomb scattering [82]. For detailed information on the application the Kalman filter in the MINER $\nu \mathrm{A}$ track and vertex reconstruction framework, see Reference $[53]$. 


\section{Adding Clusters to Tracks}

The results from the Kalman filter fit are exploited such that any clusters missing from a track may be added. For this procedure, the candidate clusters can be a supercluster, heavy ionizing, and trackable cluster. The fitted track is projected in both the upstream and downstream directions of both the start and end positions of the track. If the track projection intersects an unused cluster, then the cluster is added onto the track. For the case of the supercluster with an energy deposition greater than that of a minimum ionizing particle (MIP), the cluster (also referred to as the parent cluster) is broken apart, where a MIP fraction of energy is given to each one of its daughters. The daughter cluster with the MIP $(2.25 \mathrm{MeV} / \mathrm{cm})$ amount of energy is then placed onto the track, whereas the others are discharged for availibility by other reconstruction algorithms. In addition, the track adding technique fills in gaps in the track. In order to fill in the gap, there must be a cluster associated with that plane.

\section{Track Cleaning}

Both the hadron and muon track candidates which are created by the LongTracker are processed through a procedure commonly known as track cleaning. The track cleaning technique breaks clusters apart and removes additional energy from the track. The objective of track cleaning is to remove the energy from a track which does not originate from the particle that is being tracked. The removed energy is freed to be consumed by other tracking and/or reconstruction algorithms.

Recall that the anchor track is most likely to correspond to a muon. Thus, if the $d E / d x$ per plane is inconsistent with a muon energy loss profile, then any extra energy is removed from the anchor track. Only the superclusters are cleaned for the anchored tracks, which are assumed to correspond to hadrons. If a supercluster is close to the end of the anchored track, the cleaning procedure becomes extremely 
relaxed, meaning that more than a MIP worth of energy remains on the parent cluster. This ensures that the Bragg region of the energy loss profile remains uncorrupted.

\section{The ShortTrackers}

As mentioned above, the ShortTrackers are essential for the reconstruction of tracks with large scattering angles with respect to the longitudinal axis of the detector and particles with trajectories that spanned less than nine planes. Within the MINER $\nu \mathrm{A}$ reconstruction framework, there exists multiple of ShortTrackers, where the Anchored Short Tracker and Vertex Energy Short Tracker are utilized in succession for the analysis presented in this dissertation. These trackers can reconstruct tracks that span down to a minimum of five planes and particles with scattering angles that may range from $\left[60-80^{\circ}\right]$ and $\left[110-180^{\circ}\right]$. This is accomplished because the track formation procedure is not nearly as rigorous as the method described for the LongTracker pattern recognition algorithm. Consequently, the trackers are most likely to preform unsuccessful for events with very heavy energy activity. To avoid the reconstruction of spurious tracks, the ShortTrackers are preferably executed in a reconstruction routine which imposes various requirements on the event topology. In addition, for the analysis presented, the tracks created by the short trackers are not cleaned and the Coulomb multiple scattering is not activated in the Kalman filter.

\section{Selection of Clusters}

Before running the various pattern recognition algorithms, a list of available clusters must be supplied. For this analysis, the available clusters are determined from first drawing a geometric cone that is anchored to the event vertex. The cone consists of supercluster, heavy ionizing, and trackable clusters from the time slice. The pattern recognition techniques do not enforced extreme constraints on the cluster merging step and required no gaps in the track candidates. Therefore, using a cone to gather a list of clusters is an efficient method. Here, the superclusters are uti- 
lized effectively within the formation of tracks and clusters which extend far from the vertex and intersect the cone, are qualified as candidates.

\section{The Anchored Short Tracker}

The Anchored Short Tracker uses a Four Hit Processor track formation routine for creating tracks. The routine does not form track seeds and track candidates in separate plane views. In fact, only track seeds are produced, then are processed in a merging step to form tracks, where the formation technique is implemented similar as the procedure applied in the LongTracker. This algorithm requires that the seed contains at least four consecutive clusters with one of the following alternating plane patterns: UXVX, VXUX, XUXV, or XVXU. However, this analysis enforces the seed to consist of five contiguous clusters and imposes a strict consistency requirement to reduce the creation of spurious tracks. Here, the consistency criteria verifies that the vertex and the starting position of the anchored track are within reasonable separation distances.

\section{The Vertex Energy ShortTracker}

The Vertex Energy ShortTracker incorporates a Hough transform as an angle scan between the anchor track and selected clusters for the track formation routine [83]. First, the clusters that are very near the event vertex are removed from the input container of cluster candidates. These clusters are placed aside because their reconstructed angles can influence the Hough transform. Clusters near the vertex tend to correspond to multiple particles due to the dynamics of nuclear effects and FSIs. The remaining clusters are separated by plane view, and within each view the clusters are grouped according to their angle with respect to the anchor track. The track candidates are formed from all combinations of $\mathrm{X}, \mathrm{U}$, and $\mathrm{V}$ views from the bunched clusters, where the merging procedure is similar to the technique described earlier. The clusters near the vertex are added to a track candidate, only if the 
cluster and track candidate are in adjacent planes. In addition, the track candidates are forced to contain at least five contiguous clusters. The criteria for declaring a candidate as a track is different from the previous compatibility conditions observed in the trackers which are described above. Here, the track must pass through the anchor vertex and contain a cluster near the vertex. Thus, this algorithm grows tracks which are emerging from the anchor vertex.

\subsubsection{VERTEX RECONSTRUCTION}

The vertex reconstruction serves to link two or more tracks to a common interaction point, where the initial vertex position is approximated using the point of closest approach (POCA) procedure. For $n$ tracks $>2$, the POCA computes the vertex position for pairwise combinations and assigned a weight to each POCA. The initial vertex for $n$ tracks $>2$ is the weighted average of the POCA for all pairs. Next, the initial vertex is refitted using an adaptive Kalman filter minimization technique [84]. The routine assigns weights to the tracks using an adaptive fitter scheme, where the tracks which are incompatible with the vertex are weighted down. Thus, tracks with poor compatabilty do not influence the reconstruction of the vertex. Since majority of the signal candidates consists of two tracks emerging from the vertex, the reconstructed vertex is not required to converge because the POCA performs very well for two track events. For more details on the implementation of the fitting procedure in MINER $\nu \mathrm{A}$ see Reference [53].

\subsubsection{MUON RECONSTRUCTION}

Tracks in MINER $\nu \mathrm{A}$ that are matched to tracks or energy in MINOS are automatically assumed to be muons. The majority of the MINER $\nu \mathrm{A}$ analyses require that the muon is reconstructed and track-matched by MINOS in order to isolate their signal candidates and reconstruct the event kinematics. Recall that the analysis presented 
in this dissertation is searching for the $\nu_{\mu}$ quasielastic-like event topology that consists of a reconstructed muon track and at least one reconstructed proton track. Since this is a simple event topology and the event kinematics can be reconstructed from either the muon, the proton, or both particles. Thus, all muons that exit the detector are included in the event selection. Note that the tracking threshold of the observed proton is responsible for preventing the selection to incorporate muons that are contained within the MINER $\nu \mathrm{A}$ detector. Figure 4.22 shows the reconstructed topology for muons that originate from the $\nu_{\mu}$ quasielastic-like scattering on the Tracker region in the detector. This section summarizes the various muon topologies that are selected in the analysis.

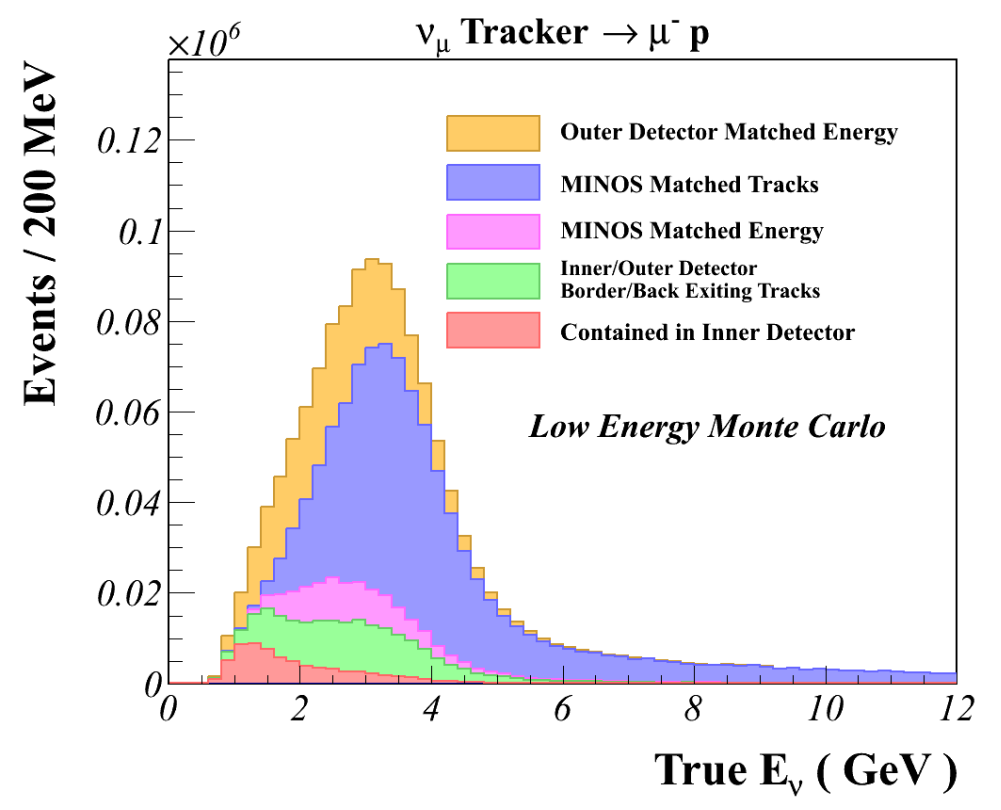

Figure 4.22. The simulated neutrino energy spectrum for the $\nu_{\mu}$ quasielastic-like channel, where the stack histograms correspond to the reconstructed muon track topology.

\section{Matching Tracks to MINOS}

MINER $\nu \mathrm{A}$ tracks that are matched to tracks or energy in MINOS are commonly referred to as MINOS-Matched Tracks or MINOS-Matched Energy(Stubs), respectively. Recall that MINOS has a magnetic field which is utilized to determine the 
charge of particles traversing the detector. The charge can only be reconstructed for the tracks in MINOS, where the MINOS-Matched track topology is the only muon topology in MINER $\nu \mathrm{A}$ which carries a charge sign. The analysis presented is insensitive to the charge reconstruction and not utilized in the selection cuts.

\section{MINOS Matched Tracks}

The conditions which the MINER $\nu \mathrm{A}$ tracks must satisfied to qualify as candidates for matching in MINOS include the following:

1. Contain at least one cluster in the last five modules of the detector (the HCAL region). Note that this criteria prevents the MINOS matching of MINER $\nu \mathrm{A}$ tracks that exit the side of the detector.

2. The track in MINOS is required to contain at least one hit in the first four instrumented planes in the MINOS detector.

3. The time separation between the MINOS and MINER $\nu \mathrm{A}$ tracks must be within a 200 ns window.

There are two compatibility tests for the track matching. The first test compares the position of the MINER $\nu \mathrm{A}$ track projected into MINOS to the MINOS track's origin, as well as comparing the position of the MINOS track projected into MINER $\nu \mathrm{A}$ to the MINER $\nu \mathrm{A}$ track end position. If the residuals of both projections meet the matching consistency criteria, then the track matching is successful and the MINER $\nu \mathrm{A}$ and MINOS track candidates are propagated to the final compatibilty test. A closest approach technique is utilized for the last consistency check, where the projected MINER $\nu \mathrm{A}$ and MINOS tracks are evaluated at a common position in space. This procedure is useful for analyzing cases when the muon scatters in the passive material between the two detectors. 


\section{MINOS Matched Energy}

The MINER $\nu \mathrm{A}$ track must satisfy the first condition that is described above to be considered for energy matching in MINOS, where the energy is characterized by hits in MINOS and is grouped into an object commonly referred to as a stub. All hits that are above an energy threshold in the MINOS detector and are not on the matched MINOS track, are available for consumption by the energy matching routine. A stub seed is created out of hits in the first six MINOS planes (with conditions on the plane views and number of hits) that occur $200 \mathrm{~ns}$ from the MINER $\nu \mathrm{A}$ track. In addition, the seed must be within $40 \mathrm{~cm}$ of MINER $\nu \mathrm{A}$ track projected into MINOS. If a stub seed is formed, then the track-energy matching scheme is successful. The next step is to extend the stub, where it is extended if a hit candidate is within a certain distance and time window from the seed's most downstream hit.

The reconstruction is also responsible for estimating the initial energy of a stub under the muon hypothesis. There are several conditions which a stub must satisfy to obtain a reconstructed energy value. These conditions have not been optimized and are quite strict because of pile up effects due to overlapping events. However, for those stubs that do meet the criteria, their initial energy is reconstructed using the range of the stub.

\section{Energy Reconstruction}

Both the MINOS tracks or stubs provide the momentum at the face of the MINOS detector. To reconstruct the muon energy at the origin of the MINER $\nu \mathrm{A}$ track, the energy lost by the muon in the detector must be taken into account. The MINER $\nu \mathrm{A}$ track can pass through the OD and/or the passive steel plane between the MINER $\nu \mathrm{A}$ and MINOS detectors before entering MINOS. An OD track-energy matching routine (describe next) determines if the track enters the OD and an extrapolation procedure checks whether the track passes through the steel plane. 
The energy loss for the track in MINER $\nu \mathrm{A}$ is calculated using the Bethe-Bloch formula [85],

$$
-\frac{d E}{d x}=K \frac{Z}{A} \frac{e^{2}}{\beta^{2}} \rho\left[\frac{1}{2} \ln \frac{2 m_{e} \beta^{2} \gamma^{2} T_{\max }}{I^{2}}-\beta^{2}-\frac{\delta}{2}-\frac{C}{2}\right],
$$

where the constant $\mathrm{K}=0.307075 \mathrm{MeVcm}^{2} / \mathrm{g}, e$ is the magnitude of the charge of the particle, $\mathrm{A}$ is the atomic mass, $\mathrm{Z}$ is the atomic number, $\mathrm{m}_{e}$ is the mass of the electron, and $\rho$ is the density of the material. Also, $\gamma=\frac{E}{M}$, where $\mathrm{E}$ and $\mathrm{M}$ are the energy

and mass of the incident particle, $\beta=\frac{\sqrt{\gamma^{2}-1}}{\gamma}$, $\mathrm{I}$ is the mean excitation energy, $\mathrm{T}_{\max }$ is maximum energy transfer to the electron for a single collision and is given by

$$
T_{\text {max }}=\frac{2 m_{e} \beta^{2} \gamma^{2}}{1+2 \gamma m_{e} / M+\left(m_{e} / M\right)^{2}},
$$

$\delta$ is the density effect correction to the ionization energy loss, and $\mathrm{C}$ is the shell correction, which is given by an empirical calculation. Many of the parameters that are used in the calculation are taken from References [86], [87]. The energy reconstruction is an iterative procedure, where the first step is to initialize the energy based on the value given by the muon in MINOS. Starting at the face of MINOS and walking upstream toward the MINER $\nu \mathrm{A}$ track's origin, the $d E / d x$ is calculated for material $m$ which the track traverses. Next, the calculated $d E / d x$ for material $m$ is added onto the total energy. Then, updated total energy is utilized to calculate the $d E / d x$ for the following material $m+1$. This continues until the track's origin is reached. The technique accounts for the energy loss of the passive materials and it is insensitive to detector inefficiencies, such as dead time.

\section{Matching Tracks to Energy in the Outer Detector}

Due to the design of the bars in the OD, extending the track reconstruction to the OD is a difficult task. Recall that the initial purpose of the OD is to serve as a hadronic calorimeter. Instead of tracking muons in the OD, a track-energy matching 
procedure is implemented, where the routine is executed before any MINOS matching algorithms. In both the upstream and downstream directions, an exiting track is projected into the OD. Note that the analyzer is free to define exiting based on the analysis needs. The clusters that are classified as superclusters, heavy ionizing, or trackable and are within the same OD tower of the projections, are selected for matching. If a cluster is within a particular distance of the projection, then the cluster is associated with the track. If at least one cluster is linked to the track, the matching of tracks to energy in the OD has succeed. This technique performs extremely well for muon track candidates that enter the OD. However, the technique needs to be revisited for quantifying its performance for the hadron track candidates.

\section{Energy Reconstruction}

For the analysis presented, the energy is reconstructed only if the OD matching routine associates clusters at the downstream end of the track. The technique is similar to the MINOS matching energy reconstruction procedure. Like the OD matching algorithm, first the track is projected into the OD, where the Kalman filter fit parameters at the end of the track is utilized. Currently, the clusters' information is excluded from determining whether the direction of the projected track may of have changed at each OD frame. However, the location of the most downstream cluster decides the end position of the projected track. If the most downstream cluster is located in the fourth story of a tower, then the projected track stops at the back of the OD frame containing this tower. Otherwise, the first OD bar that the projected track passes through that is located downstream of the last cluster is declared as the end position as illustrated in Figure 4.23. For cases similar to Figure 4.23, the energy is not reconstructed using the end position of the projected track, but a stopping position. The stopping position is defined as the halfway point between position of the most downstream cluster and the position of the first bar which the projected track passes through that is located downstream of the cluster. Now that the stopping 
position is known, the energy is reconstructed using a technique similar to the one described for the MINOS matching muon topologies.

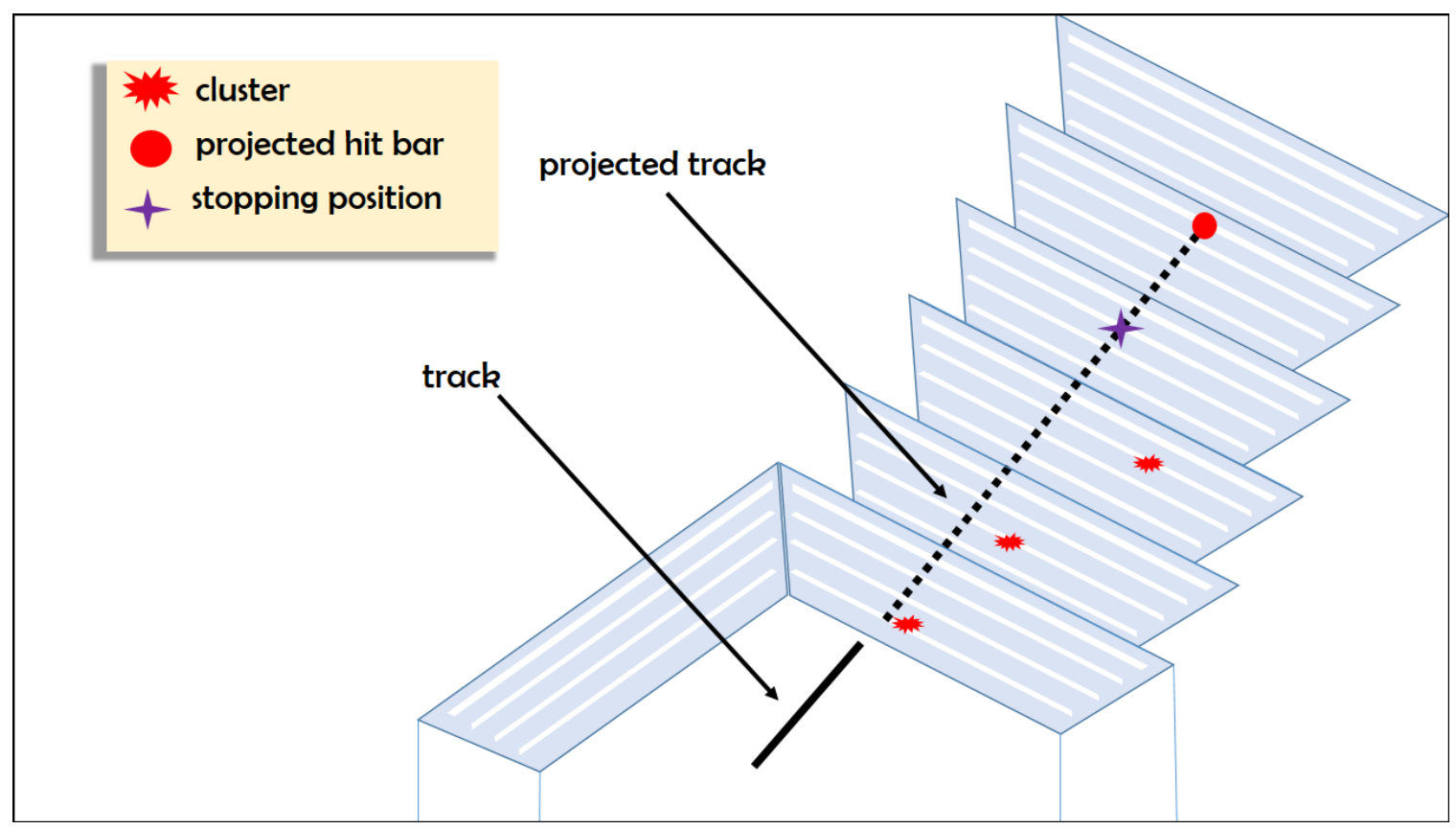

Figure 4.23. An illustration of the simple case for the OD energy reconstruction, where the energy is reconstructed using an iterative $d E / d x$ technique of the projected track. As shown, the stopping position of the projected track is determined by the most downstream cluster and the first bar which the projected track passes through.

Reconstructing the energy for particles entering the OD is not simple. The low energy muons are more likely to Couloumb multiple scatter, thus utilizing a projected track is inefficient for tracing the trajectory of the muon. In addition, there exists far more passive steel than active scintillator material, thus it is almost impossible to determine if the muon escapes or is contained within the OD. Finally, many muons do exit the OD and only a mimimum energy can be determined.

\section{Tracks that Exits MINER $\nu \mathrm{A}$}

Reconstructed tracks that are tagged as Exiting must meet the following criteria:

- not matched to tracks or energy in MINOS

- not matched to energy in the OD 
- must not be contained in the ID (contained is defined by the analysis).

Here, contained means that the reconstructed track stops before the last five modules of the detector and the end position of the track is within the MINER $\nu \mathrm{A}$ fiducial area (see Chapter 5). For these tracks, the energy is reconstructed from range under the muon hypothesis. For more details on the range implementation see Subsection 4.3.5.

\subsubsection{HADRON RECONSTRUCTION}

The $\nu_{\mu}$ quasielastic-like is a simple event topology, however the proton can rescatter in the detector, which complicates the reconstruction of the hadron. This section describes the procedure for linking together all of the observed hadron components into objects called prongs and attaching particle hypotheses to the hadron prong. Clearly, a prong is an object which holds the reconstructed objects that may correspond to a single particle, where the definition of particle can varied between the inclusive and exclusive analyses. For example, as shown in Subsection 4.3.4, a MINOS-matched track prong consists of the MINER $\nu \mathrm{A}$ and MINOS tracks with a muon particle object attached.

Table 4.3. The Nuclear Interaction Lengths of Materials in MINER $\nu$ A .

\begin{tabular}{c||c}
\hline Material & Nuclear Interaction Length $\left(\mathrm{g} / \mathrm{cm}^{2}\right)$ \\
\hline Scintillator & 81.7 \\
Carbon & 85.8 \\
Iron & 132.1 \\
Lead & 199.6 \\
\hline
\end{tabular}

\section{Hadron Formation}

The probability for the hadron to re-interact as it traverses the detector is energydependent. Table 4.3.5 shows the nuclear interaction length for materials in the 
MINER $\nu \mathrm{A}$ detector. The primary arguments for attaching the energy depositions of the hadron's daughter particles to the hadron include the following:

- Improvements to energy reconstruction,

- Better performance from the particle identification (pID) routines.

Therefore, additional stages are implemented in the reconstruction so that the hadron is fully approximated.

At this point in the hadron reconstruction, only the trajectory of the hadron has been determined, where this also includes kinked tracks. The next step is to attach clusters to the hadron, where the cluster candidates originate from the primary hadron. The type of clusters which may qualified as candidates are: supercluster, heavy ionizing, and trackable clusters. Before associating any energy with the hadron track, a spherical region is drawn around the event vertex, where the sphere has a radius of $10 \mathrm{~cm}$. The clusters within this region are placed aside from the reconstruction. This prevents free clusters near the vertex from becoming associated with the hadron track or with unattached energy in the event (see Chapter 5 for detailed information on unattached energy). Next, a geometric cone is anchored to the end position of the track, where clusters that are inside or intersect the geometric cone are attached to the hadron. Recall that hadrons can abruptly re-scatter which may result in the production of a secondary track and low energy particles around the secondary vertex. For these cases, clusters near the secondary vertex are stitched in a filament style. The tracks and clusters which represent the trajectory and energy deposition of the hadron, are utilized to form the hadron prong as indicated in Figure 4.24 .

\section{Particle Creation}

The particle identification (pID) of the hadron is determined at the analysis stage. At the reconstruction stage, the $\operatorname{track}(\mathrm{s})$ is used to create particles for the hadron 


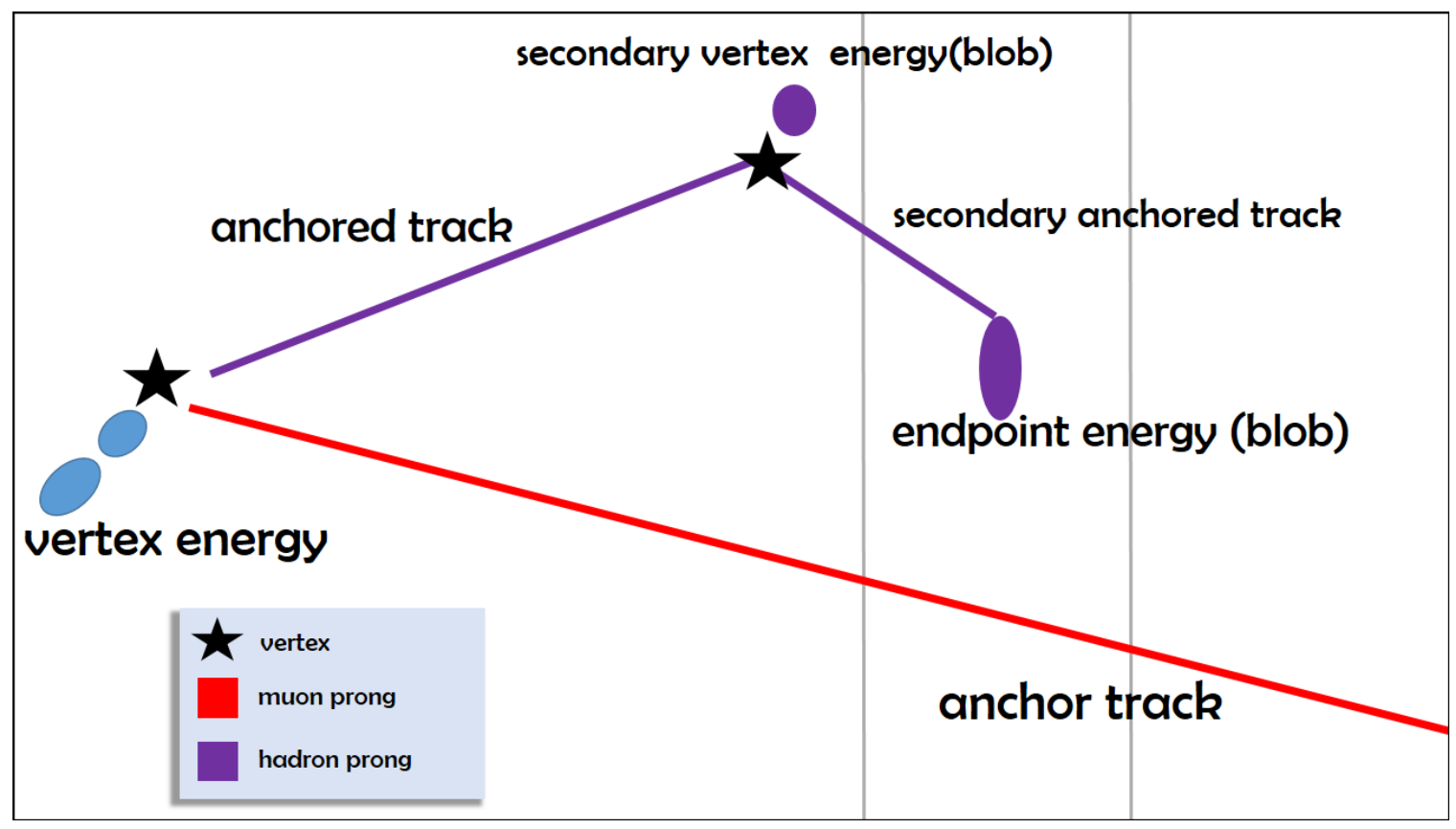

Figure 4.24. An illustration of a more complicated case for stitching together all of the components of a hadron that propagates in the detector. Un-tracked energy around the vertex is not associated with the hadron prong.

prong, where each particle has a mass and four-momentum vector. The $d E / d x$ profile and the range of the reconstructed track are utilized simultaneously for the creation of particles, where only the proton and pion particles are formed. As mentioned earlier, hadrons propagating through the detector can produce various track topologies, where only the following topologies are considered within this dissertation.

1. The trajectory of the hadron is approximated by one track, which means that the proton most likely experience little or no re-interactions in the detector.

2. The hadron undergoes a hard collision and changes direction, thus the trajectory of the hadron is represented by two tracks; a primary track and a kinked track.

For case (2), only the kinked track (the track after the interaction) is utilized in the procedure describe below. However, the primary track is taken into account for the reconstruction of the momentum vector at the primary vertex, which is described later. 
Overview of Assigning the Hadron pID

Exclusively using the track's length, a momentum range is computed for both the proton and pion hypotheses. The range provides an initial best guess approximation for the lower and upper momentum values of a stopping track. Next, in an iterative process using a $\chi^{2}$ minimization test, the procedure searches for the best fitted momentum for each particle hypothesis. This $\chi^{2}$ test compares the track's measured $d E / d x$ profile to a range of calculated $d E / d x$ profiles, where each tested profile uses a different input momentum value.

Recall that the track cleaning procedure serves to remove overlapping particles from the track. However, there are cases which show evidence that track cleaning alone cannot disentangle overlapping particles in the vertex region of a track. In addition, hadrons can undergo soft nuclear interactions such that the direction of the hadron's trajectory does not change, but the hadron leaves behind a large amount of energy deposition consistent with multiple low energy particles. Due to the various neutrino event topologies and physical characteristics of hadrons, a second iteration of the $\chi^{2}$ minimization test is processed in order to incorporate the various outlier removal techniques, which are described later in the section.

The $\chi^{2} /$ ndf values that are obtained from the first and second iterations of the $\chi^{2}$ minimization test are compared. The iteration with the smallest $\chi^{2} /$ ndf gives the reconstructed momentum for that particle hypothesis. Figure 4.25 shows an example of the track's measured $d E / d x$ profile compared with the best fitted calculated $d E / d x$ profiles for both the pion and proton hypotheses.

\section{$\chi^{2}$ Minimization Test}

The $\chi^{2}$ test compares the track's measured $d E / d x$ profile to a calculated $d E / d x$ profile based on a particle hypothesis which has a particular incident momentum, where its direction is determined by the reconstructed track. The formula for the $\chi^{2}$ is given by, 


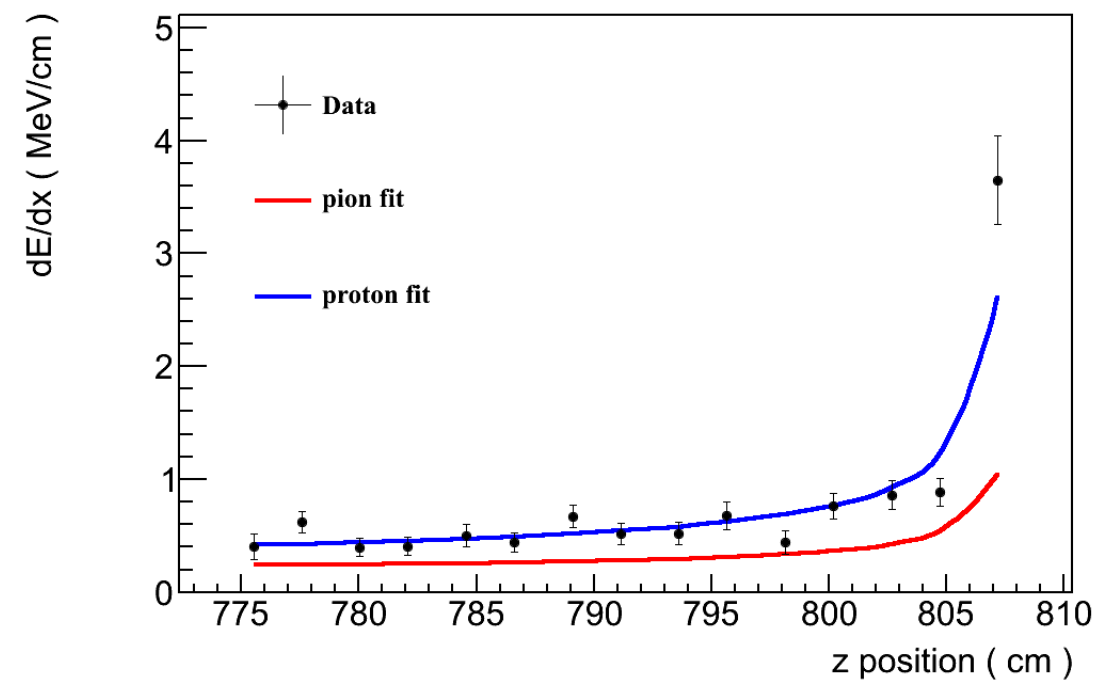

Figure 4.25. Plotted is the $d E / d x$ profiles of the reconstructed track compared with the best fitted profiles for the proton and pion hypotheses. The plot shows that the proton best describes the reconstructed track.

$$
\chi^{2}=\sum_{i=0}^{\text {nclusters }} \frac{\Delta E_{\text {meas }}-\Delta E_{\text {calc }}}{\sigma_{\text {strag }}^{2}+\sigma_{\text {photo }}^{2}+\sigma_{\text {path }}^{2}}
$$

where $\Delta E_{\text {meas }}$ is the measured energy of the cluster, $\Delta E_{\text {calc }}$ is the calculated average energy deposited in the scintillator, $\sigma_{\text {strag }}$ is the energy straggling width in the Gaussian limit and is given by,

$$
\sigma_{\text {strag }}=\xi \sqrt{\frac{1-\beta^{2}}{\frac{2 \xi}{T_{\max }}}}
$$

with

$$
\xi=\frac{K}{2} \frac{Z}{A} \frac{e^{2}}{\beta^{2}} \rho d x
$$


where $\rho d x$ is the mass thickness and the other variables are described in Subsection 4.3.4. In addition, $\sigma_{\text {photo }}$ is the calculated total photo-statistical error on the measured and predicted energy loss in the scintillator and is given by,

$$
\sigma_{\text {photo }}^{2}=\left(\frac{\Delta E_{\text {meas }}}{\sqrt{P E_{\text {meas }}}}\right)^{2}+\left(\frac{\Delta E_{\text {calc }}}{\sqrt{\text { BirksPE } / 1720}}\right)^{2}
$$

where $\mathrm{PE}_{\text {meas }}$ is the number of photo-electrons emitted by the PMT photocathode for each cluster, value 1720 is extracted from the channel-to-channel calibration, and BirksPE is the calculated light yield using Birk's Law saturation, where Birk's constant is $k_{B}=0.133 \mathrm{~m} / \mathrm{GeV}$ in the MINER $\nu \mathrm{A}$ optical model. Finally, $\sigma_{\text {path }}$ is the calculated error on the path length and given by,

$$
\sigma_{\text {path }}=0.04\left(\Delta E_{\text {calc }}-\Delta E_{\text {meas }}\right) \Delta x
$$

where the value of 0.04 is the typical theta resolution. The $\chi^{2}$ computation only calculates a $\chi^{2}$ value at a plane, if the $d E / d x$ is non-zero for both the measured and calculated energy depositions.

\section{Outlier Removal}

The outlier removal technique serves to identify the track's clusters, whose energy loss profile is consistent with multiple of particles, where the identified clusters are excluded from the $d E / d x \chi^{2}$ calculation. There exists three different schemes, where two of the techniques aims to identify clusters at the beginning of the track that should be excluded from the fit. These techniques work extremely well for the high multiplicity ( $n$ hadron tracks $>2$ ) neutrino event topologies, where the hadron tracks span more than seven planes. Recall that the analysis presented is primarily searching for only one hadron track coming out of the interaction vertex, where the hadron can spanned a minimum of five planes. Therefore, the outlier removal techniques that are mentioned above, are not incorporated in the analysis. 
An outlier removal technique is applied to hadrons that span more than ten planes. The purpose of this procedure is to identify clusters which correspond to soft collisions experienced by the hadron as it propagates through the detector. Using the fit parameters that are given by the first iteration of the $\chi^{2}$ minimization test, the scheme searches for clusters with a $d E / d x \chi^{2}$ that is $3 \sigma$ from the mean. Those clusters are tagged and excluded from the second iteration of the $\chi^{2}$ minimization test. This routine performs quite well for the proton particle.

\section{Computing a Particle Score}

The particle's score is used to determine the quality of the $d E / d x$ profile fit and the identity of the hadron track. The formula for the score is given by,

$$
\operatorname{score}_{p(\pi)}=1.0-\frac{\left(\frac{\chi^{2}}{n d f}\right)_{p(\pi)}^{2}}{\sqrt{\left(\frac{\chi^{2}}{n d f}\right)_{p}^{2}+\left(\frac{\chi^{2}}{n d f}\right)_{\pi}^{2}}} .
$$

Equation 4.3.8 shows the score computation using the $\chi^{2} /$ ndf obtained from the proton and pion $d E / d x$ fits. The score quantifies which hadron that the reconstructed track most likely resembles, assuming that the track ranges out in the detector. Therefore, the score is interpreted as a ranging out particle identification score.

\section{Optimizing the Momentum Reconstruction}

For case the where the hadron prong contains the primary and kinked tracks (see Figure 4.24), the procedure that is illustrated above, reconstructs the momentum for each given particle hypothesis at the secondary vertex. In addition, the calculated score is based completely on the $d E / d x$ profile of the kinked track. For the single track hadron prong, the momentum is reconstructed at the origin of the track. Recall that the hadron prong may contained energy that is linked to its secondary vertex and/or end position of the track. This energy is taken into account in order to 
fully reconstruct the initial momentum of the hadron prong for each given particle hypothesis. The procedure is described below.

1. For the kinked hadron prong case:

(a) If clusters are attached to the secondary vertex, then the calorimetric corrected energy of the clusters are added to the reconstructed total energy.

(b) Using the technique that is summarized for the muon energy reconstruction (see subsection 4.3.4), the energy loss by the primary track is taken into account, under the particle hypothesis of the kinked track, where the momentum is initialized based on the value obtained in step (a).

2. If clusters are attached to the end position of the track (kinked track), then the the calorimetric corrected energy of the clusters are added to the reconstructed total energy.

3. The last step accounts for the energy loss between the event vertex and the track's origin.

This is a effective technique which results in a momentum resolution of about 20-25 $\mathrm{MeV} / \mathrm{c}$ for the protons that are evaluated in the analysis presented in this dissertation. However, the overall scheme for hadron formation and particle creation must be optimized for the higher energy hadrons, where nuclear re-interactions are more likely to occur. 


\title{
SECTION 5
}

\begin{abstract}
ANALYSIS
Recall that charged current neutrino quasielastic (QE) scattering is the scattering of the neutrino from a nucleon bound in the nucleus and via the exchange of a charge vector boson, $\mathrm{W}^{ \pm}$the scattered charged-lepton changes flavor due to the nature of the weak nuclear force and the nucleon remains in it's ground state. As mentioned previously, the boson $\mathrm{W}^{ \pm}$, can be absorbed by a nucleon-nucleon correlated pair or coupled with a virtual meson which is being exchanged between two nucleons in the nucleus. The scattering nuclear physics that are not described by the independent nucleon impulse approximation result in observed enhancements in the measured QE cross section for scattering from nuclei heavier than Hydrogen. In addition, for all neutrino scattering processes that occur on nuclei with $\mathrm{A}>1$, the ejected hadrons at the scattering vertex can re-scatter or be absorbed in the nucleus. Thus, final state interactions can influence the kinematic distributions of the ejected hadrons and are responsible for non-QE events mimicking the signature of the $\mathrm{QE}$ channel. Instead of measuring the $\nu_{\mu} \mathrm{QE}$ cross section, the $\nu_{\mu}$ quasielastic-like (QE-like) cross section is measured, where QE-like is described by,

- one muon,

- no mesons,

- at least one proton.

Due to the nuclear physics summarized above, this analysis searches for event topologies with one reconstructed muon and at least one reconstructed proton. In principal, event topologies that consist of $\mu^{-}+p+p$ in the final state are allowed to enter into the signal selection. These event topologies can represent the signature of multinucleons at the scattering vertex or elastic collisions that occur between the
\end{abstract}


struck nucleon and a spectator nucleon in the nucleus. This chapter presents the analysis of the $\nu_{\mu}$ quasielastic-like differential cross section, $\mathrm{d} \sigma / \mathrm{dQ}^{2}$ on a polystyrene scintillator $(\mathrm{CH})$ target.

\subsection{SELECTING $\nu_{\mu}$ CHARGED CURRENT EVENTS}

Subsections 4.3.4 and 4.3.5 provide an overview of the procedure for reconstructing tracks in the MINER $\nu \mathrm{A}$ detector. As shown in Figures 4.20 and 4.24, tracks trace the trajectories of particles propagating through the detector and additional reconstruction algorithms are utilized for linking tracks together and associating unused clusters to the tracks. This section summarizes the cuts which are applied for selecting the $\nu_{\mu}$ charged current (CC) events with at least two tracks emerging from the neutrino interaction vertex, where one of the tracks is associated with the muon and the other tracks are declared as hadrons. The cuts are applied sequentially and include the following:

1. Large fiducial volume. The event vertex is required to be within a fiducial volume that is slightly larger than the MINER $\nu \mathrm{A}$ standard fiducial volume which is described by the final cut.

2. Events with isolated tracks. The event must not have a track that is isolated, meaning that the track is not associated with the event vertex. Event topologies with isolated tracks usually represent the signature of a neutrino interaction with a neutral particle in the final state.

3. Number of reconstructed tracks. There must be at least two tracks coming out of the reconstructed neutrino interaction vertex.

4. Selecting the muon prong candidate. If a track is matched to a track or hits in the MINOS detector, then it is automatically declared as the muon 
candidate. Currently, tracks matched to hits in the OD are also declared as muons. Therefore, if the event has more than one track tagged as an ODMatch prong, then the event is discarded. For all other cases, the prong with the longest track in the event is identified as the muon candidate, where the longest track must be exiting the detector.

5. Selecting the hadron track candidates. The prongs which contains tracks that are not identified as muons, are declared as the hadron candidates. These hadron prongs must not be tagged as Forked, which is defined in Subsection 4.3.5. In addition, each hadron prong must contain reconstructed particles which provide the four-momentum vector and particle score for both the proton and pion hypotheses. A cut is not applied to the stopping position for these candidates. Based on the event topology that is described for selecting the muon prong candidate, by default, the hadron prong is assumed to be contained if it is tagged as a non-ODMatch prong.

6. The fiducial volume. The reconstructed primary vertex is required to be within the MINER $\nu \mathrm{A}$ standard fiducial volume, which is defined as the following:

- $598.0 \leq \mathrm{Z}_{\text {vertex }} \leq 842.2 \mathrm{~cm}$

- The transverse position is within a hexagonal apothem of $85 \mathrm{~cm}$, where an apothem is the distance from the center to the midpoint of one side of the hexagon.

This cut ensures that the neutrino interaction occurs within the scintillator tracking region of the detector and excludes events where the neutrino interacts on a lead collar which borders a scintillator plane.

Table 5.1 shows the remaining fraction of events for both the data and simulated events after applying each cut that is described above. The differences that are observed in the event rate between the data and Monte Carlo originate at the number of 
tracks requirement. This may be due to GENIE overestimation of the pion production rate, particularly the $\Delta^{++}$resonance reaction. For example, the proton pID score distribution (see Figure 5.2) shows that the Monte Carlo over-predicts the number of events with pions.

Table 5.1. The Event Rate for Selecting $\nu_{\mu}$ Charged Current Events.

\begin{tabular}{l||c|c}
\hline \hline Cuts & Data & Monte Carlo \\
\hline Big Fiducial Volume & $100 \%$ & $100 \%$ \\
$\mathrm{~N}_{\text {isolated-tracks }}=0$ & $89.7 \%$ & $89.2 \%$ \\
$\mathrm{~N}_{\text {tracks }} \geq 2$ & $51.5 \%$ & $57.7 \%$ \\
$\mathrm{~N}_{\text {muon-prong }}=1$ & $36.7 \%$ & $42.6 \%$ \\
$\mathrm{~N}_{\text {hadron-prong }} \geq 2$ & $31.4 \%$ & $36.3 \%$ \\
Fiducial Volume & $21.4 \%$ & $26.3 \%$ \\
\hline \hline
\end{tabular}

\section{The Number of Tracks Discussion}

Due to the nuclear dynamics beyond the impulse approximation and the proton propagation in the nucleus, this analysis is sensitive to the number of protons that is observed in the final state. As a result, there is not a criteria on the number of reconstructed protons. However, due to the applied analysis cuts (describe next) and the tracking resolution, the majority of the events which enter into the $\nu_{\mu}$ QE-like selection consists only of two tracks, where one track represents the muon and the other is a proton. Furthermore, the tracking threshold prevents the reconstruction of single protons at very low $\mathrm{Q}^{2}$. There are small fraction of events (based on scanning a selection of events) where multi-protons in the final state which are aligned backto-back (a signature of SRCs) enter into the signal selection. However, these protons are often reconstructed on a single track. This is because low energy nucleons with their momentum vector aligned back-to-back or those that are overlapping in detector space cannot be separated by the current reconstruction algorithms. In the higher $\mathrm{Q}^{2}$ region, more than one proton track can be reconstructed. However, these protons are required to be identified correctly, which implies that all of these high energy protons 
range out in the detector. After the application and discussion of each analysis cut, the number of tracks distribution is presented in order to fully understand the effect of each applied cut on this observable. Figure 5.1 shows the number of tracks after each of the cuts which are discussed above.
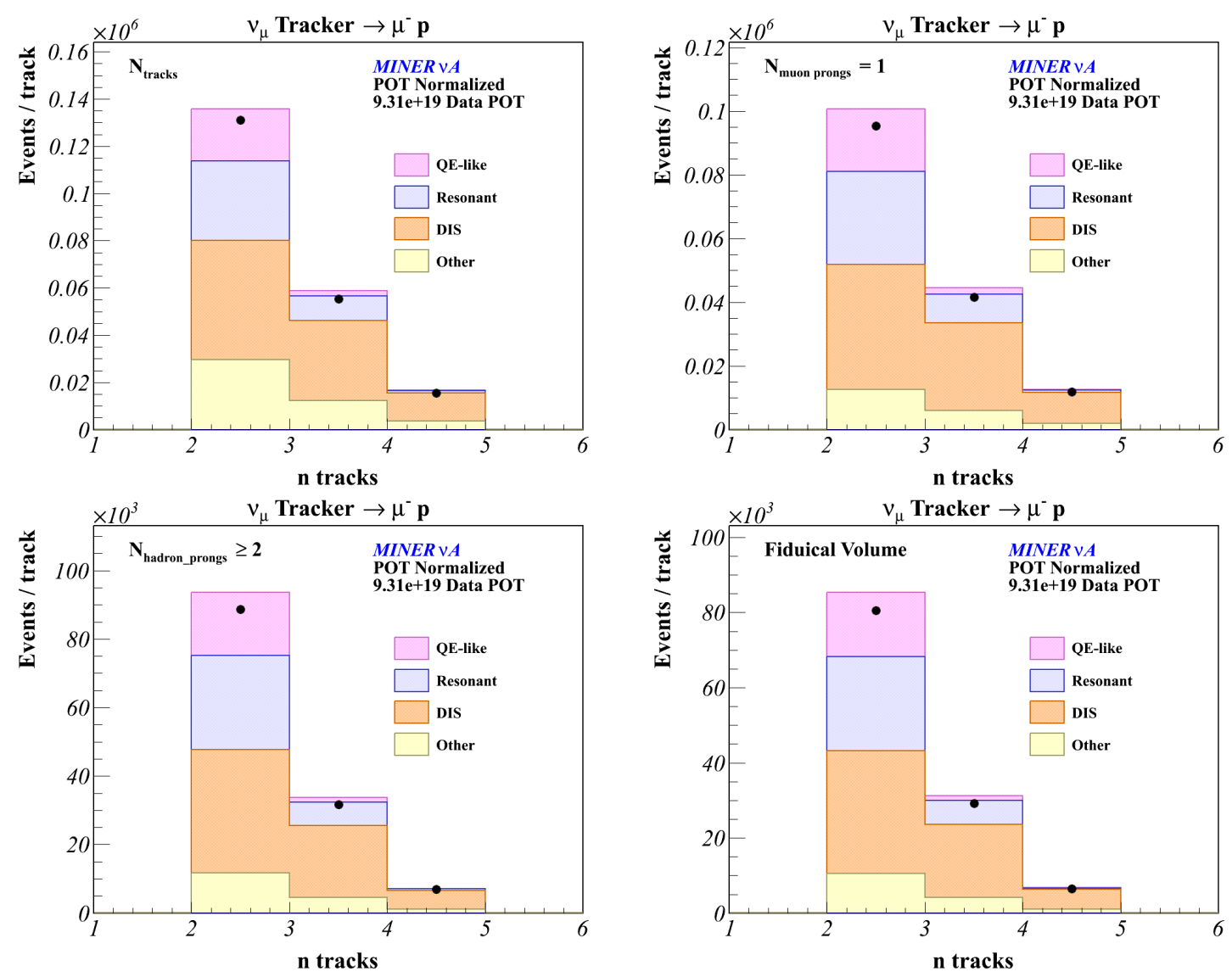

Figure 5.1. Absolutely normalized data and Monte Carlo comparison of the number of tracks after applying each cut that was discussed earlier. One of the tracks is identified with the muon candidate and other tracks are declared as the hadron candidates. The Monte Carlo histograms are characterized by the signal (QElike) and the non-signal (Resonant, DIS, other) events.

\subsection{ISOLATING THE $\nu_{\mu}$ QUASIELASTIC-LIKE EVENTS}

The $\nu_{\mu}$ QE-like events have a simple event topology. The majority of the reconstructed events consists of a muon prong and one reconstructed proton prong. This 
section describes the applied analysis cuts which isolate the $\nu_{\mu}$ QE-like candidates in the tracker region of the $\mathrm{MINER} \nu \mathrm{A}$ detector.

\subsubsection{THE APPLIED ANALYSIS CUTS}

Following the event topological selection cuts which are discussed above, there are three additional cuts applied to perform effectively and efficiently for signal event selection based on Monte Carlo studies. Each cut has been optimized sequentially for the isolation of the $\nu_{\mu}$ QE-like candidate events. The cuts are summarized below.

\section{Proton Identification (pID)}

The first cut serves to select events where all of the hadron candidates are reconstructed as protons. As mentioned in Subsection 4.3.5, $d E / d x$ is utilized for particle identification by fitting the measured energy loss profile of the hadron track candidate to both the proton and pion calculated energy loss profiles. From each fit, a $\chi^{2} / \mathrm{ndf}$ value is obtained, and the $\chi^{2} /$ ndfs are used to create a pID score for each hadron hypothesis (see Equation 4.3.8). The pID score determines which ranging out particle (proton or pion) that the hadron prong candidate energy profile most resembles. The

pID score for the proton hypothesis is shown in Figure 5.2, where all of the hadron prongs in the event must have a pID score greater than 0.35 units to pass the proton pID consistency criteria.

As mentioned previously, the track reconstruction cannot disentangle hadron particles overlapping in all three plane views (X, U, and V). Recall that this analysis does not apply any additional routines to prevent these track topologies from influencing the pID score calculation. This is because the presented analysis relies heavily on the short track reconstruction algorithms which reconstruct protons that can span a minimum of five planes and may scatter at large angles relative to the detector longitudinal axis. Since these additional routines are excluded from the from the particle's score calculation, the spectrum of the pions in the pID score distribution (see 


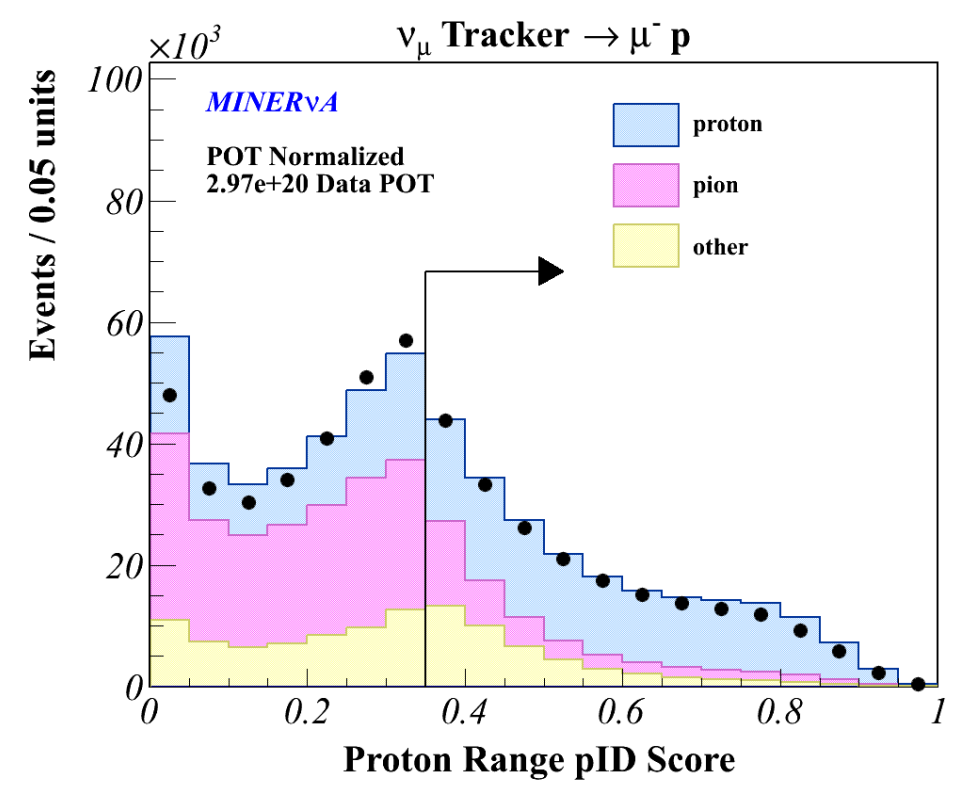

Figure 5.2. Absolute normalized data and Monte Carlo comparison of the proton pID score, which is interpreted as a particle ranging out score. The histograms are categorized by the Geant4 primary particle truth information. The discrepancy between the data and Monte Carlo is primarily due to the pion production cross section in GENIE.

Figure 5.2), does not peak at zero. In spite of this effect, Figure 5.2 shows that pID score is an efficient variable for discriminating protons from pions for the purpose of this analysis. Figure A.20 shows the proton pID score distribution after applying all of the analysis cuts.

Figure 5.3 shows the number of tracks distribution after applying the pID cut. The events with four or more tracks are removed, as well as majority of the threetrack events, because these generally contain a pion track. Based on the Monte Carlo, the majority of the remaining three-track events are likely backgrounds in which the final state pion has not been tracked.

\section{Removing Events with Heavy Activity}

The invariant hadronic mass (W) of the genuine QE event is equal to the mass of the recoil nucleon $\left(\mathrm{M}_{N}\right)$. Thus, events with $\mathrm{W} \gg \mathrm{M}_{N}$ produce heavy activity 


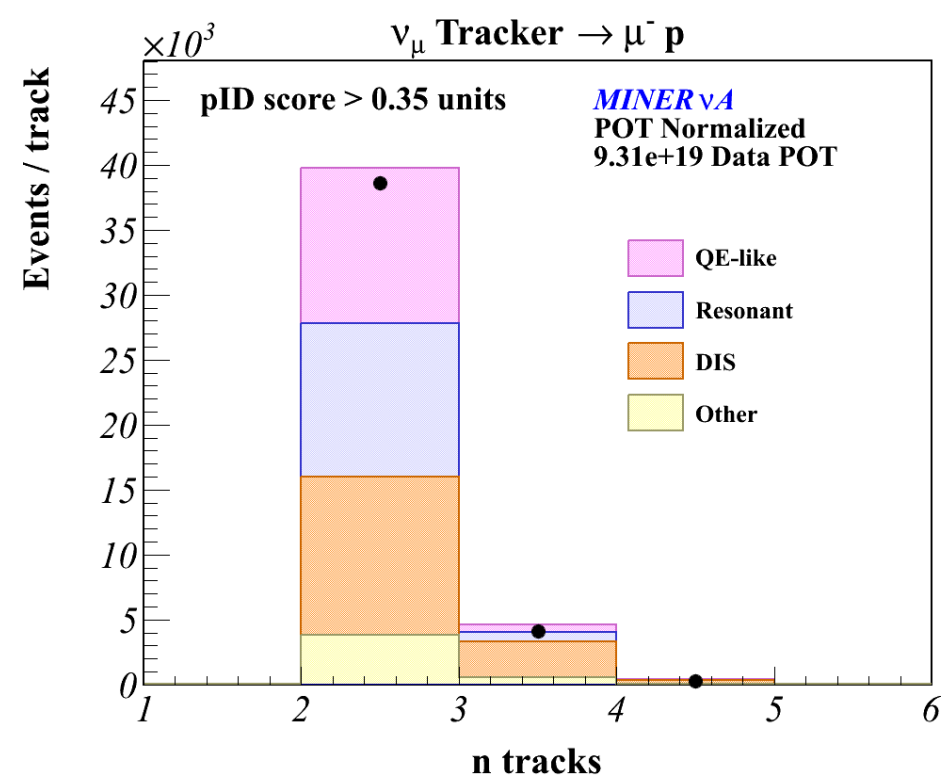

Figure 5.3. Absolutely normalized data and Monte Carlo comparison of the number of tracks after applying the pID cut. The pID cut essential removes all of the events with more the three tracks, which are dominated by the resonant and DIS events.

in the detector which represents a hadronic final state with higher multiplicities of outgoing hadrons. Therefore, such events are more likely not to originate from a $\nu_{\mu}$ QE-like reaction. The next cut is applied to an observable known as the unattached visible energy which serves to remove event topologies that exhibit heavy activity. The unattached visible energy is defined as the sum of the visible energy in the Tracker or ECAL regions located outside of a geometric sphere (radius $=10 \mathrm{~cm}$ ) drawn around the event vertex and which is not associated with the muon or proton prong. Figure 5.4 shows an illustration of the unattached visible energy observable.

Recall that the nuclear dynamics beyond the independent nucleon impulse approximation is not accurately simulated in the GENIE model that is used for this analysis. Consequently, the energy inside of the geometric sphere around the event vertex is sensitive to the mis-modeling of the multinucleon contribution to the $\nu_{\mu}$ 


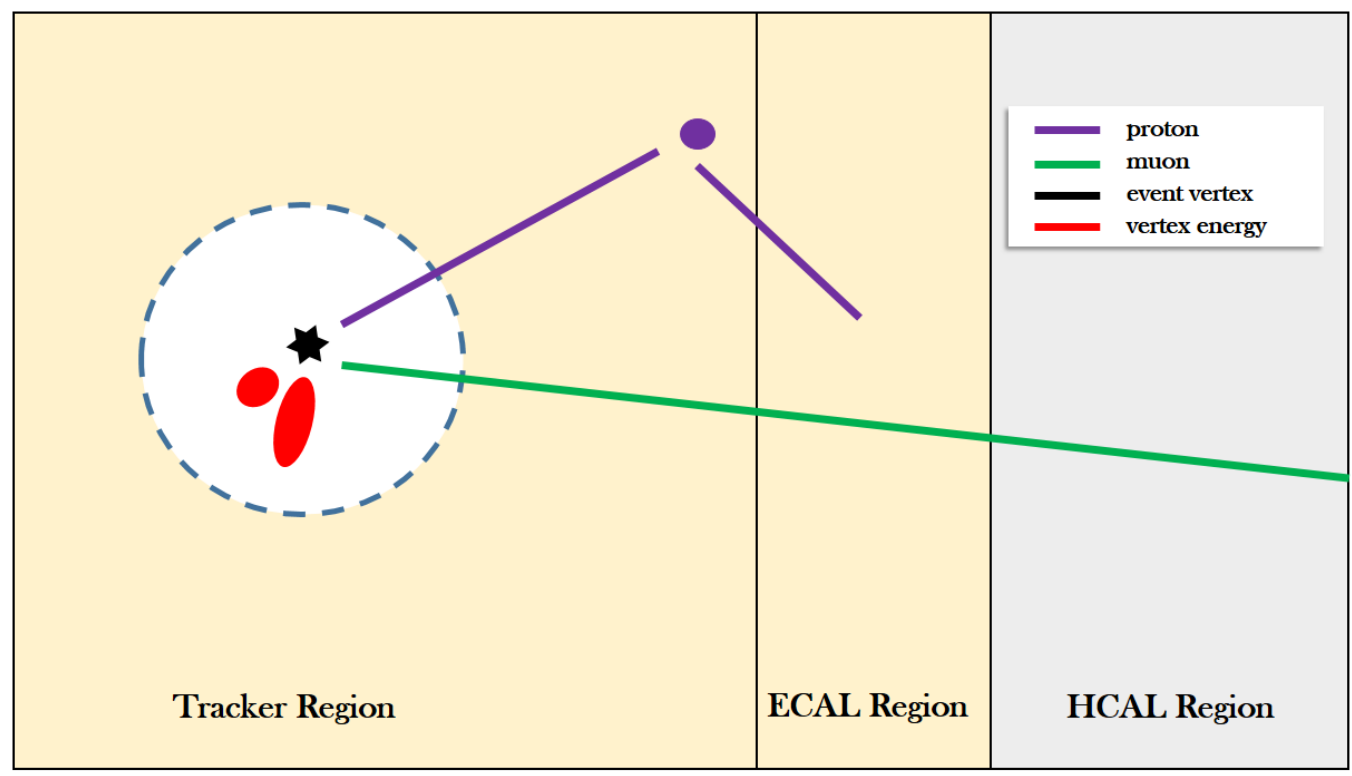

Figure 5.4. Illustration of the detector regions in which the unattached visible energy is located. This illustration does not display any unattached visible energy. However, the energy that falls outside of the sphere with radius $=10 \mathrm{~cm}$ and is unassociated with a primary particle, makes up the unattached visible energy.

QE-like cross section. Thus, the unattached visible energy (see Figure 5.5) excludes vertex energy in the summation.

In general, the low $\mathrm{Q}^{2} \nu_{\mu} \mathrm{QE}$-like events produce no or very little unattached visible energy, $\mathrm{E}_{\text {attach }}^{\text {vis }}$, relative to the events at higher values of $\mathrm{Q}^{2}$. The cut on $\mathrm{E}_{\text {unattach }}^{\text {vis }}$ is applied as function of $\mathrm{Q}^{2}$, where $\mathrm{Q}^{2}$ is calculated using only the proton kinematics. The purpose for analyzing the cut as a function of the $\mathrm{Q}^{2}$ that is computed from the proton is explained in Subsection 5.2.2. Figure 5.6 displays the 2-dimensional distributions in unattached energy and $\mathrm{Q}_{\mathrm{QE} \text {,proton }}^{2}$ for the simulated events, as well as the $\mathrm{Q}_{\mathrm{QE} \text {,proton }}^{2}$ dependent cut. For the event generated at higher values of $\mathrm{Q}^{2}$, the proton's energy is more likely to be mis-reconstructed. Therefore, these events migrate into the low and moderate $\mathrm{Q}_{\mathrm{QE} \text {,proton }}^{2}$ bins. As a result, there is less $\mathrm{E}_{\text {attach }}^{\text {vis }}$ observed at the higher $\mathrm{Q}_{\mathrm{QE} \text {,proton }}^{2}$ bins. 

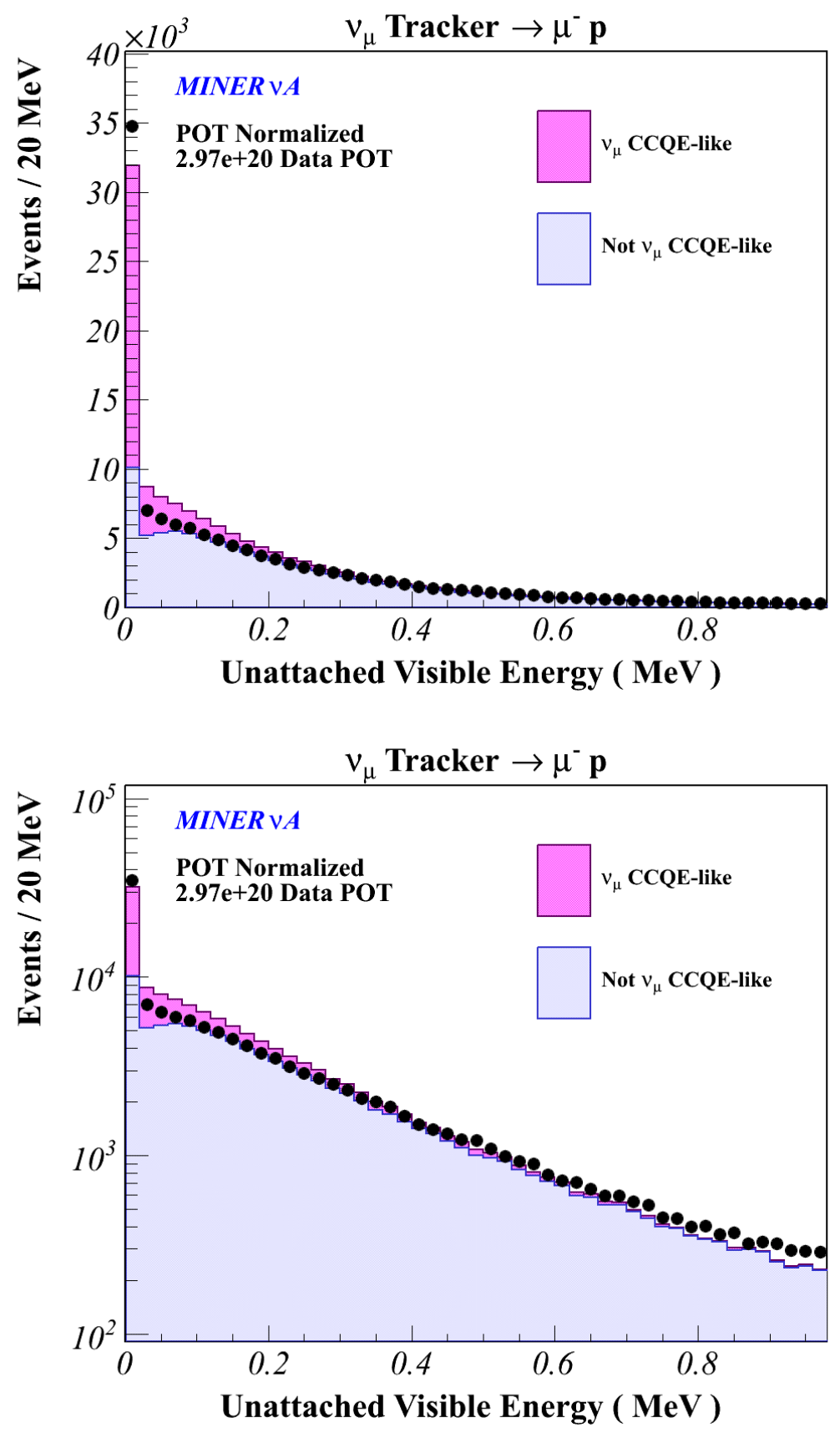

Figure 5.5. Absolute data and Monte Carlo comparison of the unattached visible energy on a linear (top) and log vertical (bottom) axis. The histogram contents are characterized by the analysis signal definition. 

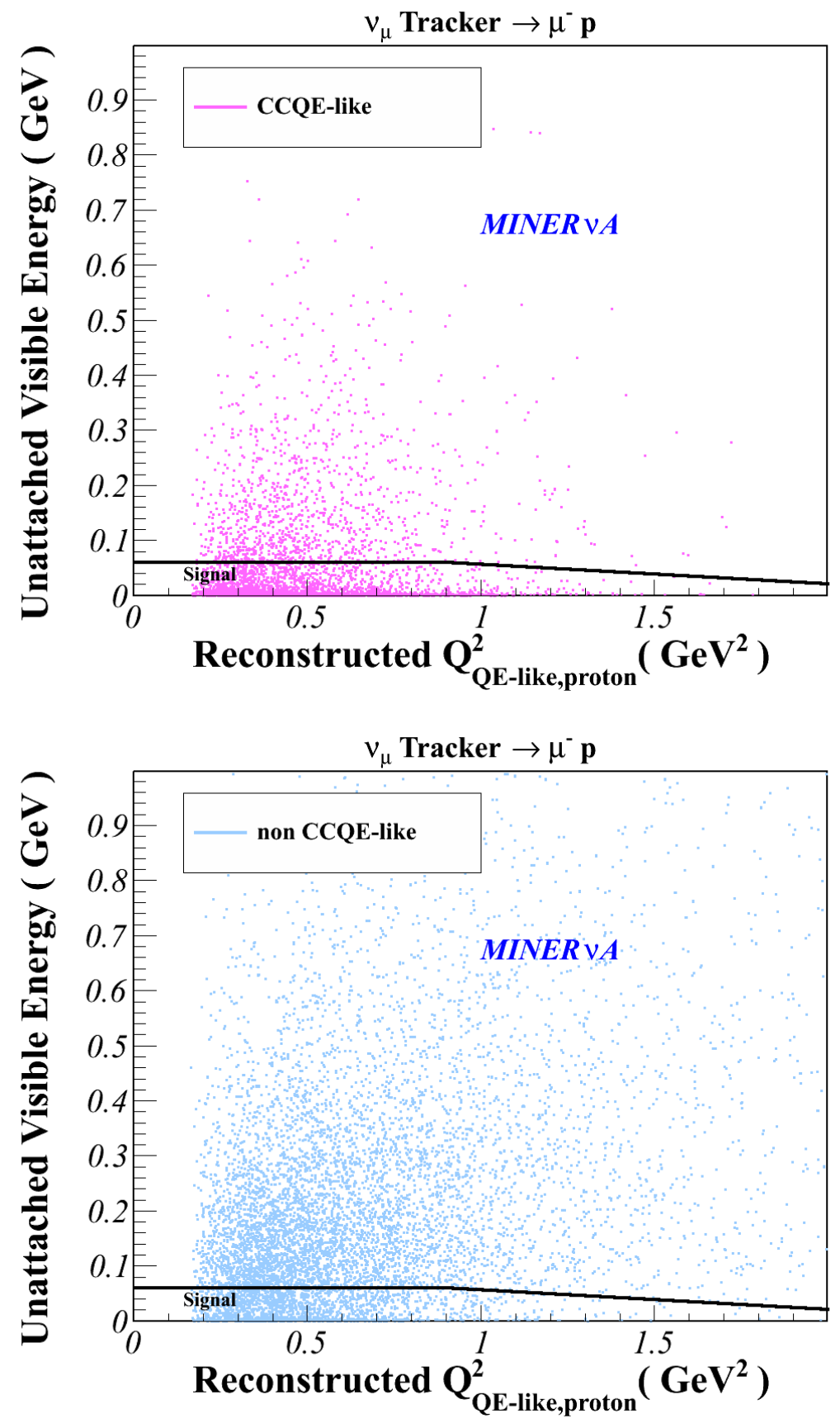

Figure 5.6. Distribution of simulated events in $\mathrm{E}_{\text {unattach }}^{\text {vis }}$ and $\mathrm{Q}_{\mathrm{QE} \text {,proton }}^{2}$ for the signal (top) and non-signal (bottom) events, along with the $\mathrm{Q}_{\mathrm{QE} \text {,proton }}^{2}$ dependent cut. 
The plot in Figure 5.7 illustrates the number of tracks remaining in the event selection after applying the 2-dimensional $\mathrm{E}_{\text {attach }}^{\text {vis }}$ cut. Notice that majority of the inelastic events have been removed and the remaining event selection consists of a very small fraction of the three-track candidates.

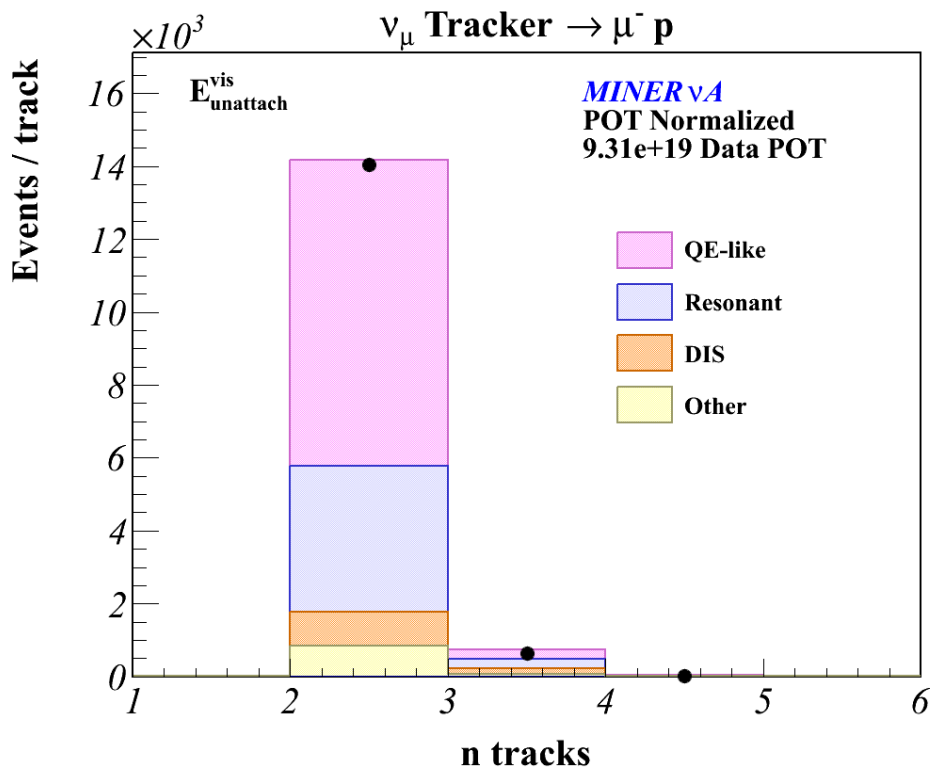

Figure 5.7. Absolutely normalized distribution for the data and Monte Carlo comparison of the number of tracks after applying the unattached visible energy cut. This cut primarily removes events that originate from the DIS process.

\section{Removing Events with Soft Pions}

The final cut applied vetoes events with a soft pion near the event vertex. These events originate primarily from resonance production. Recall that the charged pion decays via the weak nuclear interaction into a muon and neutrino, $\pi^{\mp} \rightarrow \mu^{\mp}+\nu_{\mu}\left(\bar{\nu}_{\mu}\right)$. The dominant decay modes of the muons are,

$$
\begin{gathered}
\mu^{-} \rightarrow \mathrm{e}^{-} \nu_{\mu} \bar{\nu}_{e} \\
\mu^{+} \rightarrow \mathrm{e}^{+} \bar{\nu}_{\mu} \nu_{e} .
\end{gathered}
$$

with a mean lifetime of $\tau=2.6 \times 10^{-8}$ sec. The electrons which are produced via the muon decay are called Michel electrons. An algorithm that searches for Michel 
electrons is utilized to identify events with soft pions. The Michel electron is created from unused clusters that are within the same NuMI beam spill of the event vertex, where these clusters must satisfy the following conditions,

- the total energy of the cluster is greater than $0.8 \mathrm{MeV}$,

- the position window of the Michel electron candidate with respect to the interaction vertex is,

$\left|\mathrm{Z}_{\text {displacement }}\right| \leq 12.5 \mathrm{~cm}$

$\left|\mathrm{T}_{\text {displacement }}\right| \leq 17.5 \mathrm{~cm}$, where $\mathrm{T}$ is the transverse position $\mathrm{X}$, $\mathrm{U}$, or $\mathrm{V}$.

- the discriminator on the FEB must of have fired.

The Michel electron is created from various combinations of clusters:

- a single cluster in one of the $\mathrm{X}, \mathrm{U}$, or $\mathrm{V}$ plane views (1 view Michel),

- a collection of two clusters with one of the following alternating patterns, XU, XV, or UV (2 view Michel),

- a group of clusters with at least one cluster in each of the plane views (3 view Michel).

For the analysis presented, an event is identified with producing a Michel electron if a Michel electron is found near the event vertex. If the event satisfies the Michel electron consistency requirement then the event is vetoed. Figure 5.8 shows the data and Monte Carlo histogram comparisons for the Michel electron tag, where a Michel electron is not found(is found) for the events in the first(second) bin, respectively. Note that the performance of the Michel electron algorithm on soft pion events has not been extensively studied for the analysis that is presented in this dissertation. The studies are primarily performed on rock muons that stopped in the MINER $\nu \mathrm{A}$ detector. These studies do not precisely describe the Michel algorithm performance for pion events. The performance of the Michel electron finder for stopping pions is currently being extensively examined.

Figure 5.9 shows the number of tracks distribution after the final analysis cut. The Michel electron veto does remove a small fraction of the three-track events. As 


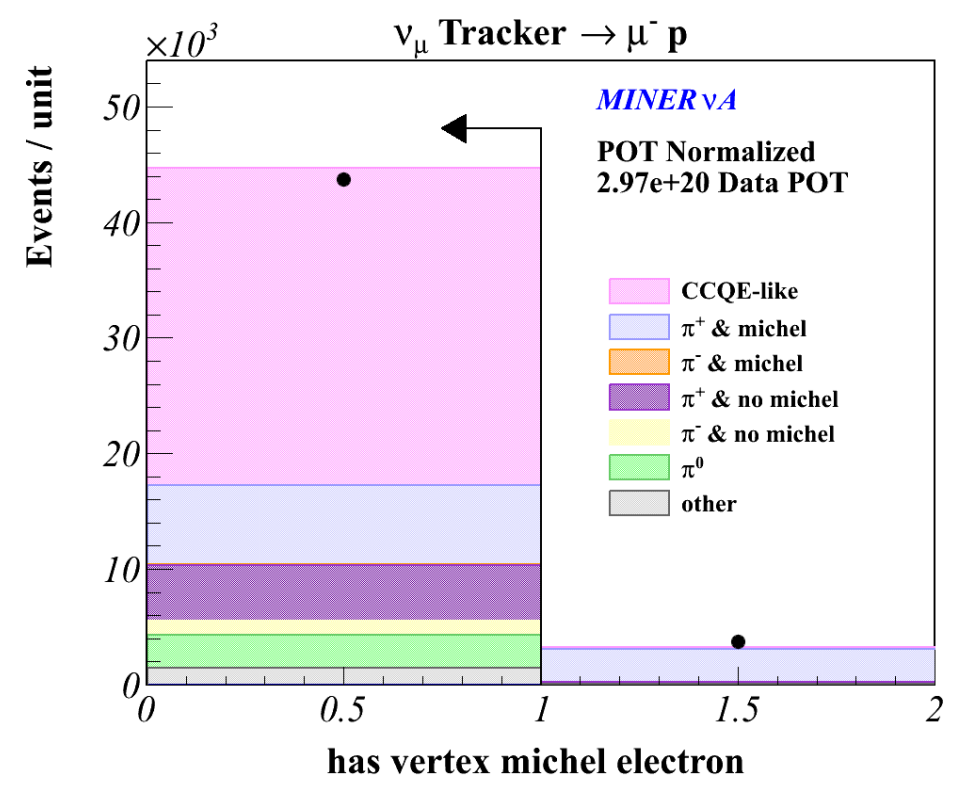

Figure 5.8. Histogram of the absolutely normalized data and Monte Carlo events with and without a Michel electron. The $\pi^{-} \mathrm{s}$ are more likely to be captured by a nucleus and the $\pi^{0} \rightarrow \gamma \gamma$. Using the Geant4 truth information, the histogram shows the fraction of $\pi^{+}$events with a Michel electron.

mentioned earlier, after applying all cuts, the $\nu_{\mu}$ QE-like event selection is dominated by the two-track events.

\section{Summary of the Analysis Cuts}

Table 5.2 displays the cumulative event rate for both the data and Monte Carlo after applying each cut. Recall that after requiring that the event vertex falls within the MINER $\nu \mathrm{A}$ standard fiducial volume, the data and Monte Carlo event rates are $21.4 \%$ and $26.3 \%$, respectively. The discrepancy that is observed between the data and Monte Carlo event rates after applying the proton pID range score condition, shows that the Monte Carlo overestimates the pion production event rate.

\subsubsection{THE $\nu_{\mu}$ QUASIELASTIC-LIKE CANDIDATES}

As mentioned previously, this analysis accepts events with various types of muon topologies, which are described in Subsection 4.3.4. Figure 5.10 shows the data and 


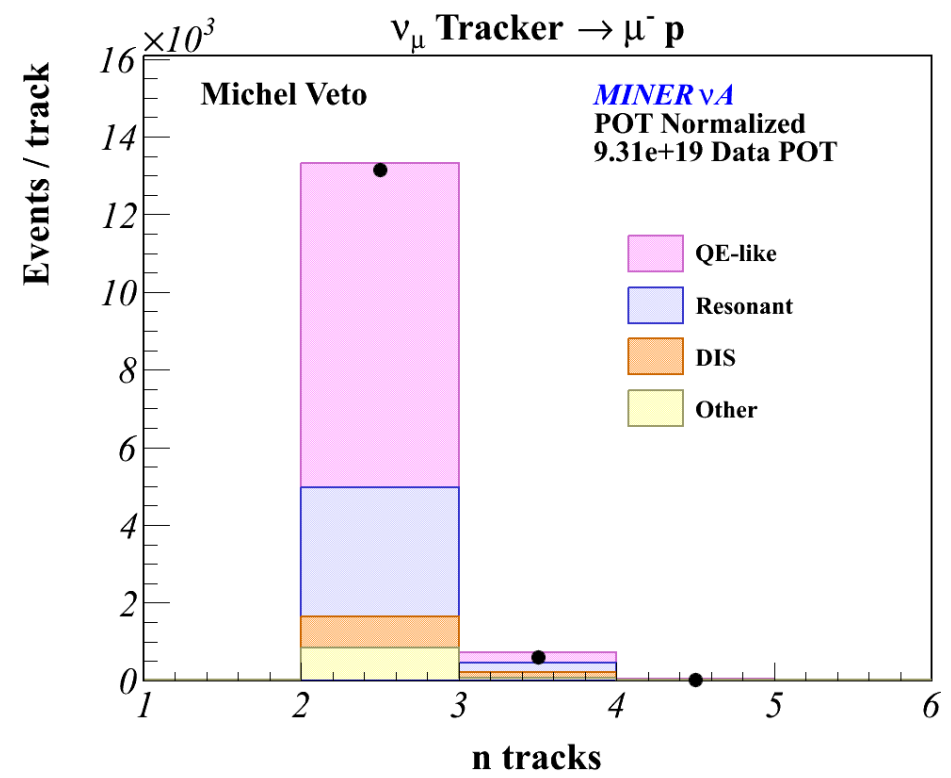

Figure 5.9. Absolutely normalized data and Monte Carlo comparison of the number of tracks after the vetoing event with a Michel electron.

Table 5.2. The Event Rate for Isolating the $\nu_{\mu}$ QE-like Candidates.

\begin{tabular}{l||c|c}
\hline \hline Analysis Cuts & Data & Monte Carlo \\
\hline \hline pID range score $\geq 0.35$ & $7.7 \%$ & $9.4 \%$ \\
$\mathrm{E}_{\text {unattach }}^{\text {vis }}\left(Q_{Q E-\text { like,proton }}^{2}\right)$ & $3.0 \%$ & $3.6 \%$ \\
Michel Electron Veto & $2.8 \%$ & $3.4 \%$ \\
\hline \hline
\end{tabular}


Monte Carlo distribution of the muon angle with respect to the beam axis for the QElike candidates. The distribution make-up is categorized based on the reconstructed topology of the muon, where Table 5.3 summarizes the percentage contribution from each topology.

Table 5.3. The Muon Topology Rate for the Simulated Events.

\begin{tabular}{l||c}
\hline \hline Muon Topology & $\mathrm{N}_{\text {Events }}(\%)$ \\
\hline \hline MINOS Matched Tracks & $53.8 \%$ \\
MINOS Matched Energy & $0.3 \%$ \\
OD Matched Energy & $32.1 \%$ \\
Exiting & $13.7 \%$ \\
\hline \hline
\end{tabular}

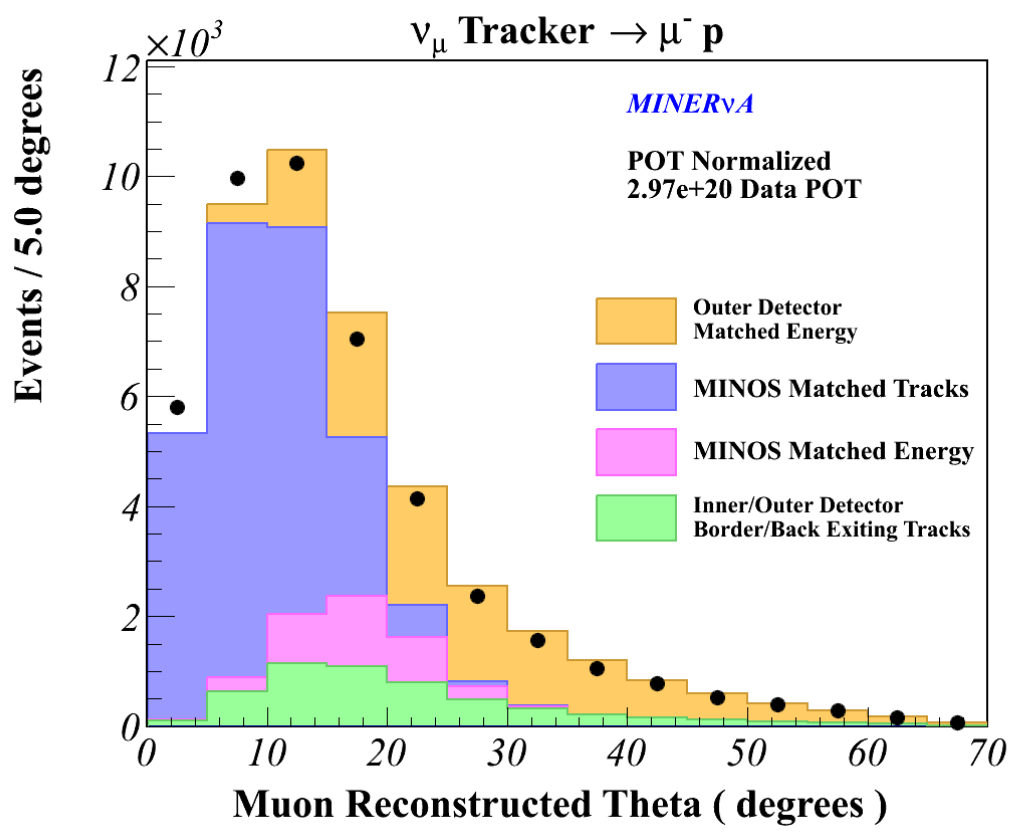

Figure 5.10. Distribution of the muon angle with respect to the beam axis absolutely normalized for the data and Monte Carlo comparison. See the text and Table 5.3 for more information describing the plot.

The distribution (Figure 5.10) illustrates the MINOS angular acceptance for muons exiting the MINER $\nu \mathrm{A}$ detector, where muons with scattering angles greater than $20^{\circ}$ are typically not reconstructed by MINOS. Recall that the reconstructed energy is poor for muons that are not track-matched by MINOS, as indicated in the 


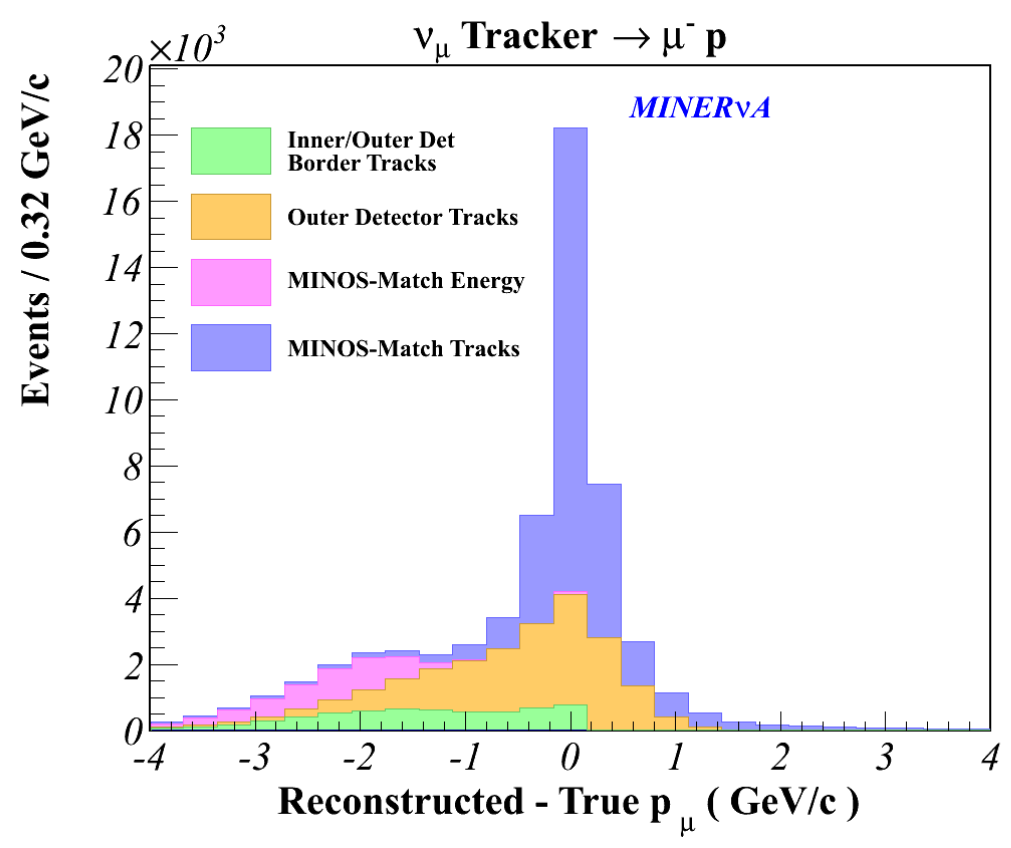

Figure 5.11. Plotted is the muon momentum residual. The histogram is categorized by the reconstructed topology of the muon. The momentum reconstruction performs the best for the muons that are reconstructed and track-matched by MINOS.

muon momentum residual distribution in Figure 5.11. However, the resolution for the momentum reconstruction of protons generated in the Tracker Region is generally better than $20 \mathrm{MeV} / \mathrm{c}$, as indicated by the RMS of the narrower Gaussian in the proton momentum residual distribution in Figure 5.12. The low-side tail of the residual histogram consists primarily of the high $\mathrm{Q}_{\mathrm{QE} \text {,proton }}^{2}$ events, which are momentum mis-reconstructed due to re-interactions in the detector. The high-side tail is composed mainly of the low $\mathrm{Q}_{\mathrm{QE} \text {,proton }}^{2}$ events, where the momentum is reconstructed too high due to the mis-reconstruction of the event vertex. The mean offset of $\sim-13$ $\mathrm{MeV} / \mathrm{c}$ comes from a bias in the $d E / d x$ fitting procedure. Because the momentum of protons are generally reconstructed better than the momentum of muons, the $\mathrm{Q}^{2}$ is reconstructed from the proton kinematics assuming QE scattering from a free nucleon at rest, via the formula 


$$
\begin{aligned}
Q_{Q E, \text { proton }}^{2} & =-q^{2} \\
& =\left(p_{\text {neutron }}^{\mu^{\prime}}-p_{\text {proton }}^{\mu}\right)^{2} \\
& =\left(M^{\prime}\right)^{2}-M_{\text {proton }}^{2}+2 M^{\prime}\left(T_{\text {proton }}+M_{\text {proton }}-M^{\prime}\right),
\end{aligned}
$$

where $\mathrm{T}_{\text {proton }}$ and $\mathrm{M}_{\text {proton }}$ are the kinetic energy and the mass of the proton. Here, $\mathrm{M}^{\prime}$ is defined as

$$
M^{\prime}=M_{\text {neutron }}-B E
$$

where the value of the binding energy, $B E=34 \mathrm{MeV}$ is based on that used for previous MINER $\nu \mathrm{A}$ QE analyses [1] and [2]. Equation 5.2.1 shows that the calculated $\mathrm{Q}_{\mathrm{QE} \text {,proton }}^{2}$ depends only on the kinetic energy of the proton. Figures 5.13 and 5.14 show examples of event displays of QE-like candidates for data events, which are created by the Arachne event display software [88].

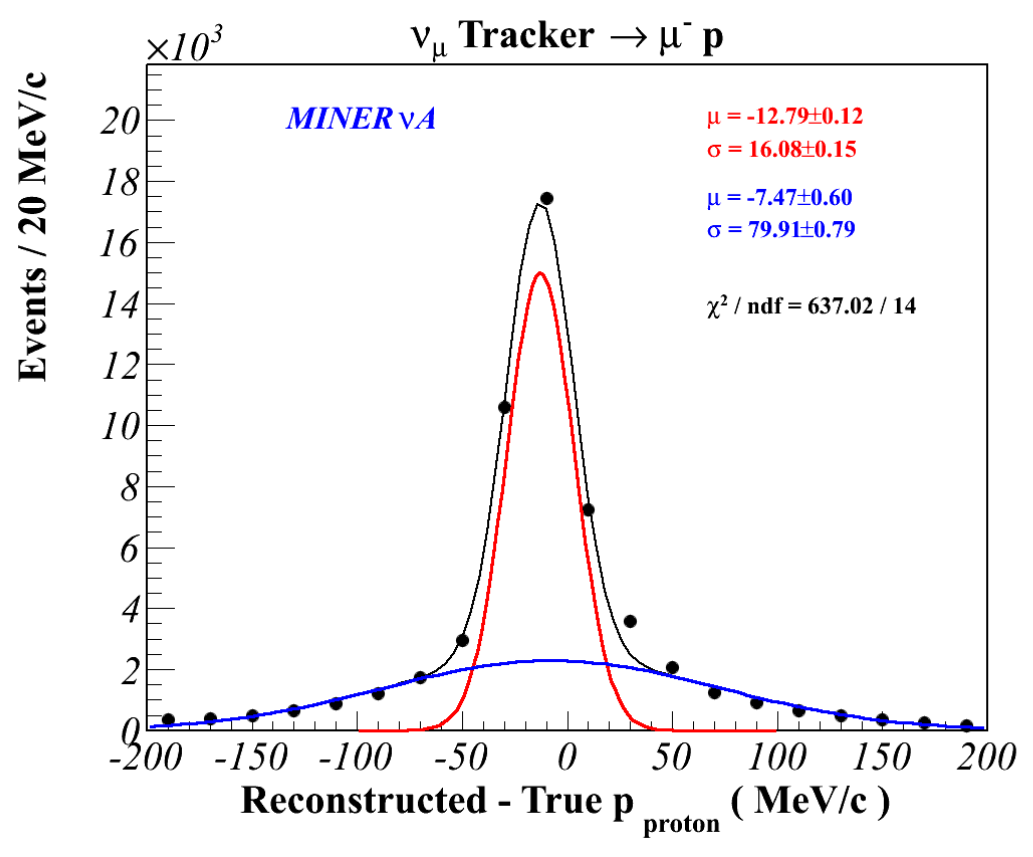

Figure 5.12. Histogram of the proton momentum residual for protons in the Tracker region of the MINER $\nu \mathrm{A}$ detector. 

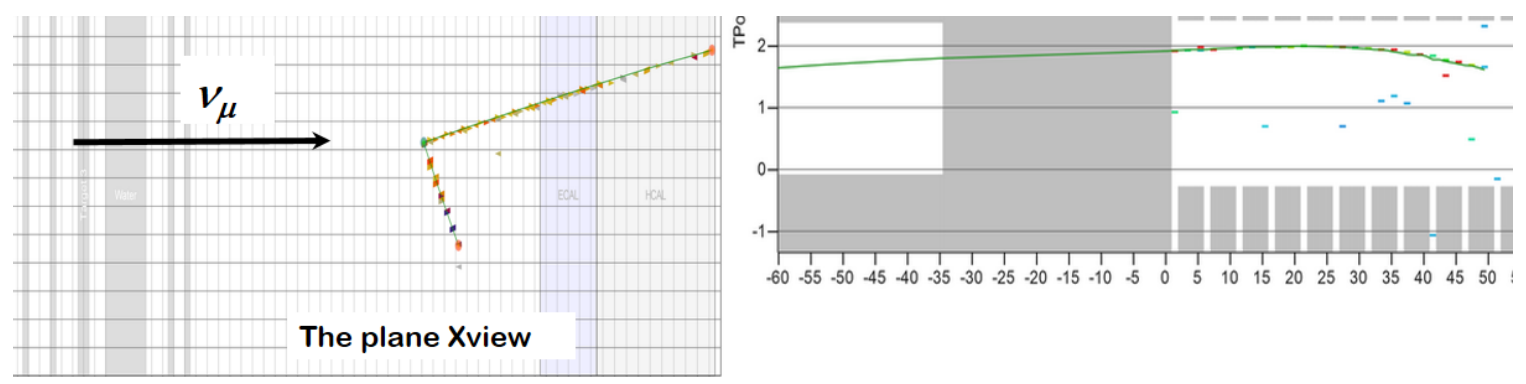

Figure 5.13. The muon exits the MINER $\nu \mathrm{A}$ detector and is matched to a track in MINOS.
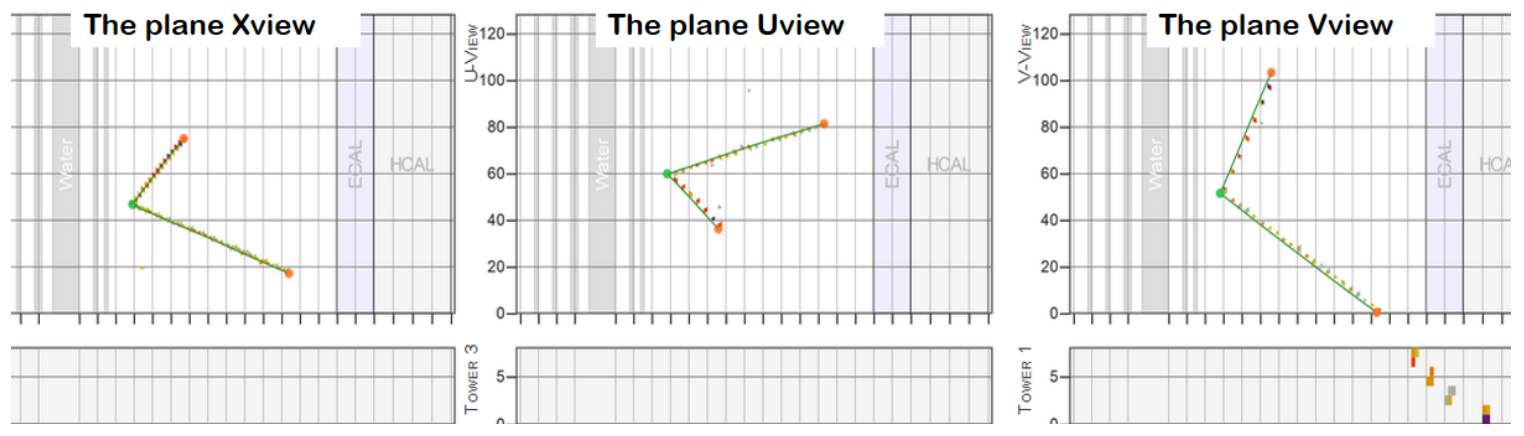

Figure 5.14. The muon is matched to energy in Outer Detector.

The $\mathrm{Q}_{\mathrm{QE} \text {,proton }}^{2}$ spectrum for the QE-like candidates is shown in Figure 5.15. Note that there are not any entries for $\mathrm{Q}_{\mathrm{QE} \text {,proton }}^{2} \leq 0.15 \mathrm{GeV}^{2}$, which is a result of the tracking threshold, where reconstructed tracks must span at least five planes. This results in an effective threshold of $\sim 450 \mathrm{MeV} / \mathrm{c}$ for the forward going protons. The spectrum drops rapidly as $\mathrm{Q}_{\mathrm{QE} \text {,proton }}^{2}$ becomes larger, which is another significant feature. The probability that the proton will re-scatter increases with energy, thus higher energy protons are more likely to undergo additional nuclear interactions in the detector. Due to these re-interactions, the protons are more likely to be mis-identified which result in the decrease in the pID efficiency. In additional, higher energy protons tend to enter the OD. These events are difficult to momentum analyze, thus are excluded from the analysis and contribute to the rapid decline in the efficiency. Both the shape and absolute error summaries for the $\nu_{\mu}$ QE-like candidates are shown 

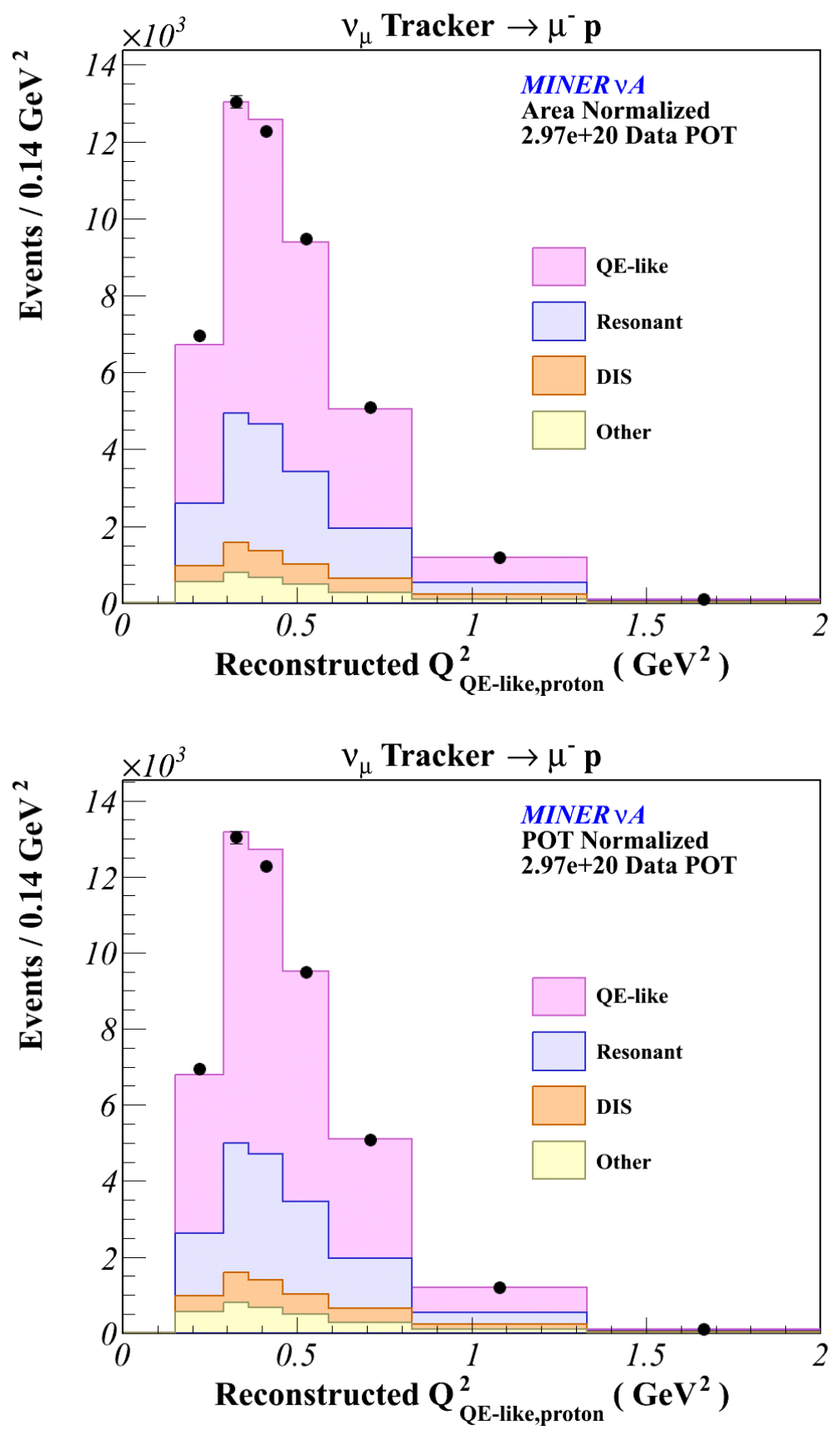

Figure 5.15. The $\nu_{\mu}$ QE-like candidates data and Monte Carlo area normalized (top) and absolute normalized (bottom) comparisons. The histogram make-up is characterized by the QE-like channel which includes the GENIE QE, Resonant, and DIS events with a final state that satisfies the defined signal. The non QE-like events are characterized by their GENIE definition. 
in Figure 5.16, where an overview of each systematic uncertainty is summarized in Section 5.4.
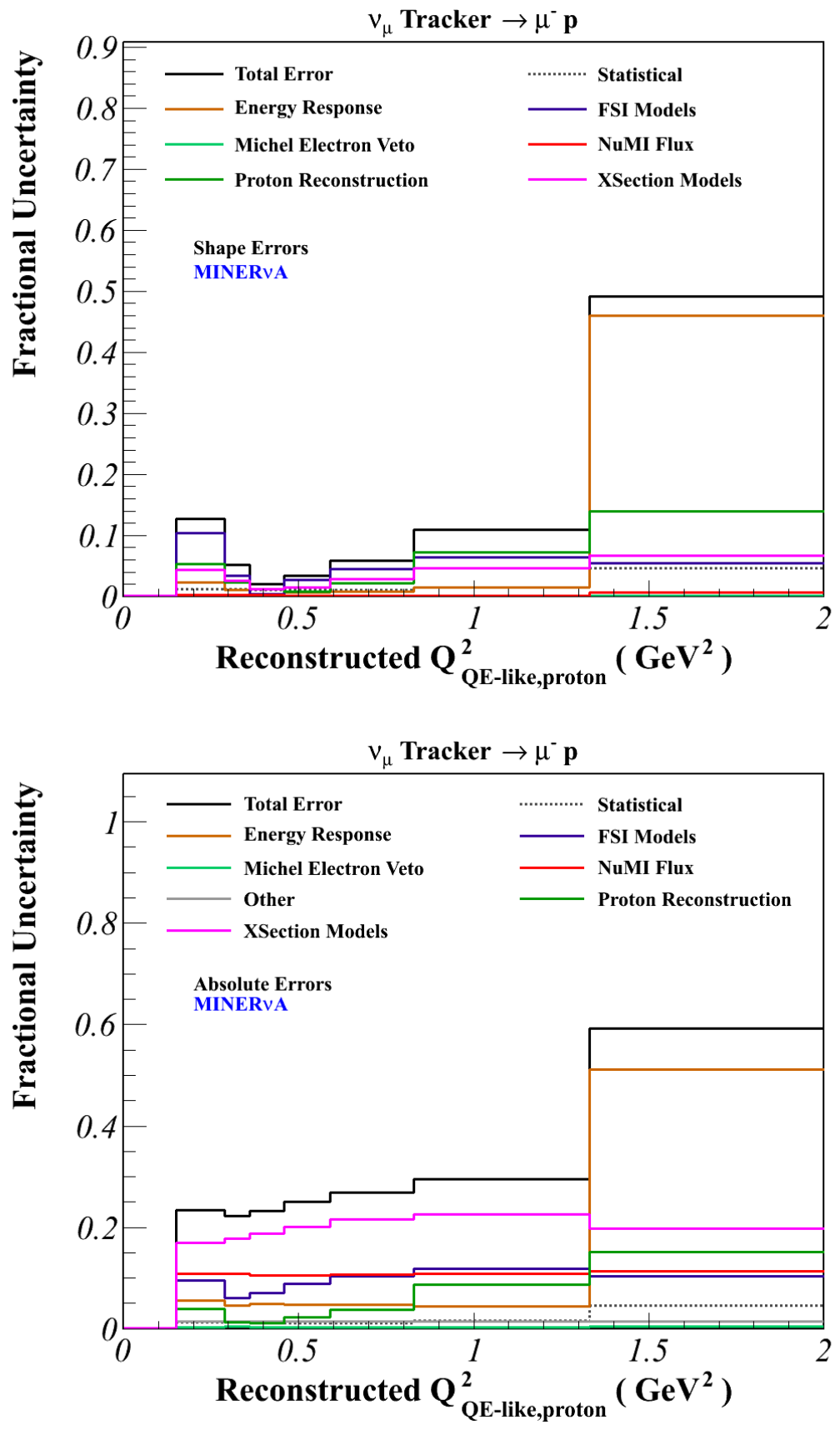

Figure 5.16. The shape (top) and absolute (bottom) error summaries for the $\nu_{\mu}$ QE-like candidates. For all plots presented in this dissertation, the shape errors corresponds to the errors on the area normalized distributions, where the shape error definition is given in Subsection 5.4 


\subsection{MEASURING THE CROSS-SECTION}

The formula to produce the flux-integrated differential cross section per $\mathrm{Q}^{2}$ bin $i$, is given by

$$
\left(\frac{d \sigma}{d Q^{2}}\right)_{i}=\frac{1}{\Phi T} \frac{1}{\Delta Q^{2}} \frac{\sum_{j} U_{i j}\left(N_{j}^{\text {data }}-N_{j}^{b k g d}\right)}{\epsilon_{i}}
$$

where

- $\Phi$ is the flux integrated over the range of $\mathrm{E}_{\nu}=[0 ., 100.] \mathrm{GeV}$,

- $\mathrm{T}$ is the number of target nucleons,

- $\Delta \mathrm{Q}^{2}$ is the size of the bin width,

- $\epsilon$ is the signal efficiency and acceptance,

- $\mathrm{U}_{i j}$ is the unfolding function which converts the reconstructed $\mathrm{Q}^{2}$ to the Monte Carlo calculated $\mathrm{Q}^{2}$,

- $\mathrm{N}_{j}^{d a t a}$ is the number of data events per bin $j$,

- $\mathrm{N}_{j}^{b k g d}$ is the number of constrained background events per bin $j$.

Equation 5.3.1 shows that there are a number of computational steps that must be preformed to convert the $\nu_{\mu}$ QE-like candidates in Figure 5.15 to a cross section measurement. This section provides a description of each of those steps.

\subsubsection{BACKGROUND SUBTRACTION}

The first step towards producing a cross section measurement is to subtract off the backgrounds from the data, $\mathrm{N}^{d a t a}-\mathrm{N}^{b k g d}$. There are several types of backgrounds which are known to contaminate the signal. These backgrounds include: rock muon events, neutrino interactions that occur outside of the fiducial volume, and the non QE-like neutrino interactions. Based on event scanning, the background from the rock muon events is expected to be less than $0.1 \%$ because of the number of tracks criteria and the proton pID condition. Recall that the width of a scintillator plane 
triangular strip is $1.7 \mathrm{~cm}$ with a base of $3.5 \mathrm{~cm}$. The vertex $\mathrm{X}, \mathrm{Y}$, and Z resolutions for the QE-like signal is approximately $3.4 \mathrm{~mm}, 3.6 \mathrm{~mm}$, and $4.6 \mathrm{~mm}$, respectively. Therefore, the background from the non-fiducial events is assumed to be negligible. For the analysis presented, only the non QE-like neutrino interaction background is taken into consideration for the background subtraction procedure.

The non $\nu_{\mu}$ QE-like backgrounds originate primarily from low multiplicity resonant production and deep inelastic scattering with a small contribution from the non $\nu_{\mu} \mathrm{CC}$ channel. To estimate the non QE-like backgrounds in the data, a data driven technique is implemented, which utilizes the Monte Carlo to predict backgrounds event rates. The Monte Carlo predicts that the non QE-like backgrounds predominately originate from the resonant and DIS productions. Therefore, the non $\nu_{\mu} \mathrm{QE}-$ like backgrounds are separated into two groups, the resonant and DIS plus others. These backgrounds are constrained utilizing a bin-by-bin extrapolation procedure, where background scale factors are extracted for the two-component backgrounds. The technique for constraining the backgrounds is described below, where each step is applied sequentially.

1. First, the sidebands are selected. A sideband is defined as a region that is outside of the signal region. Recall that the second analysis cut is on the $\mathrm{E}_{\text {unattach }}^{\text {vis }}\left(\mathrm{Q}_{\mathrm{QE} \text {,proton }}^{2}\right)$ observable. This analysis isolates four sidebands in the $\mathrm{E}_{\text {unattach }}^{\text {vis }}\left(\mathrm{Q}_{\mathrm{QE} \text {,proton }}^{2}\right)$ observable, as shown in Figure 5.17, where the Michel electron veto has been applied.

2. The next step is to extract scale factors from each of the sidebands. Figure 5.18 shows the data to Monte Carlo comparisons for each sideband distribution. A bin-by-bin correction technique is utilized to extract these background scale factors for each sideband, where the procedure requires that the data and Monte Carlo are perfectly matched. As a result, the extracted scale factors are 

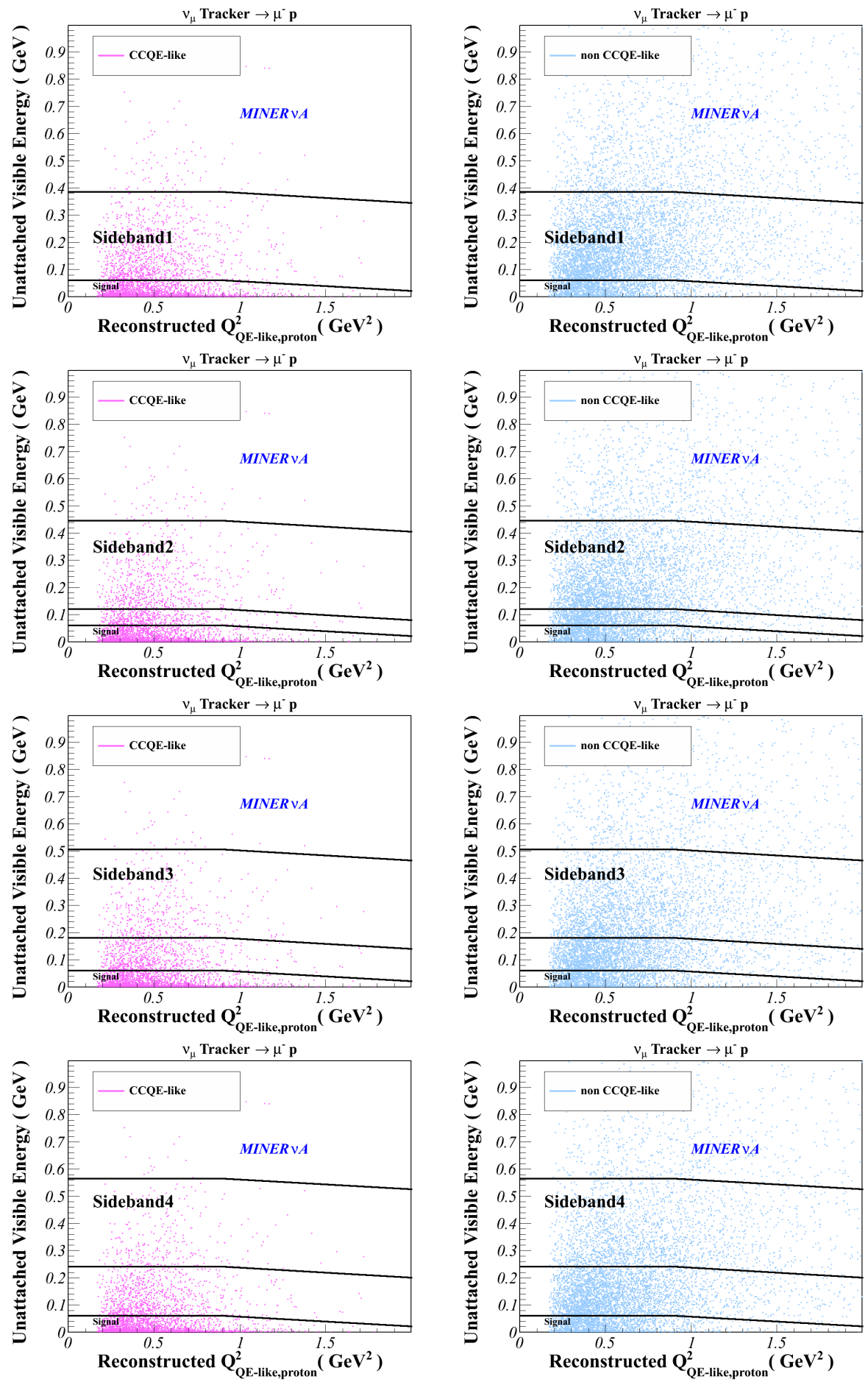

Figure 5.17. The signal (left) and non-signal (right) simulated events for each sideband. The histograms illustrate the upper and lower cut boundaries for each sideband. 

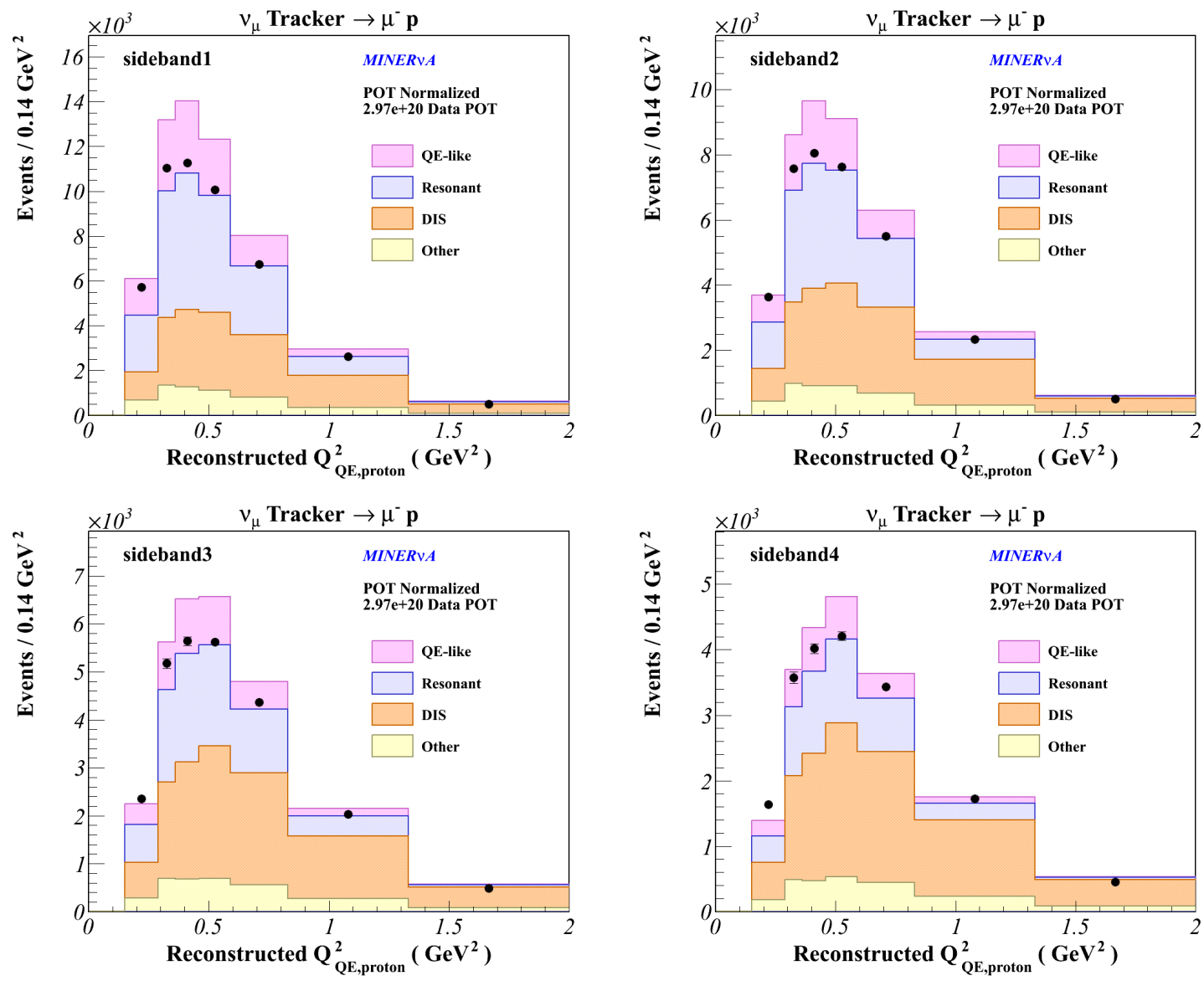

Figure 5.18. Data and Monte Carlo absolute comparisons for each sideband. The (top) sideband1/sideband2 and (bottom) sideband3/sideband 4 are characterized by the $\nu_{\mu}$ QE-like signal and GENIE definition of the backgrounds. 
the weights which force the data and Monte Carlo event rates to be equal, and are calculated from

$$
w_{i}^{b k g d}=\frac{N_{i}^{\text {data }}-N_{i}^{Q E-l i k e}}{N_{i}^{\text {nonQE-like }}},
$$

where $\mathrm{N}_{i}^{\text {data }}, \mathrm{N}_{i}^{Q E-l i k e}$, and $\mathrm{N}_{i}^{\text {nonQE-like }}$ are the number of data, Monte Carlo QElike, and Monte Carlo non QE-like events per bin $i$, and $\mathrm{w}_{i}^{b k g d}$ is the scale factor per bin $i$. Note that in Equation 5.3.2, the signal events are subtracted from the sideband data distributions, which assumes that the Monte Carlo prediction of the number of QE-like events is correct. The uncertainty of this dependence is reflected in the systematic on the background subtraction procedure.

Figure 5.19 shows the results of the extracted scale factors for each sideband. Sideband 1 is the closest to the signal region, while sideband 4 is the furthest away. As the sideband region moves further from the signal region, the scale factors move closer to unity. This shows that the Monte Carlo describes the data more accurately for the sidebands that are further from the signal region. Also, as sideband $1 \rightarrow$ sideband 4 , the overall event rate decreases and the shape of the spectrums broaden. Consequently and as expected, the sidebands that are located further away are less likely to describe the shape of the background in the signal region. The differences between the shapes in the sidebands and background in the signal region are due to the different fractional contributions from the $\mathrm{N}^{*}$ resonance states and DIS events. Therefore, it is unwise to utilize a single sideband to constrain the QE-like backgrounds.

3. The final step is to extrapolate scale factors for the two-component backgrounds using the extracted scale factors from each sideband. The extrapolated scale factors do not depend on the $\mathrm{Q}_{\mathrm{QE} \text {,proton }}^{2}$ shape differences (that are discussed above) between the sidebands and the background in the signal re- 


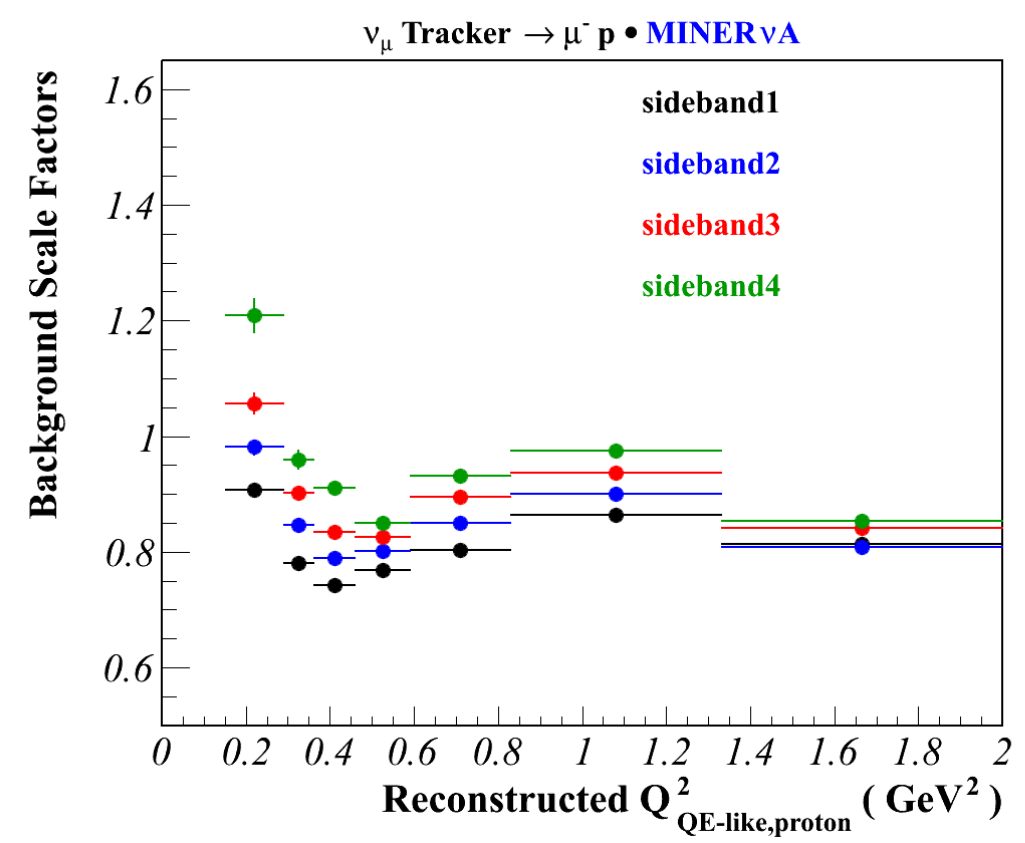

Figure 5.19. Extracted scale factors for each of the sideband, where the data to Monte Carlo event rate differ for each sideband.

gion. The technique leverages on the following Monte Carlo information which is provided per sideband.

- $\left(\mathrm{N}_{\text {Total }}^{\text {bkgd }}\right)_{i} \equiv$ the total number of Monte Carlo background events for bin $i$

- $\left(\mathrm{N}_{R e s}^{b k g d}\right)_{i} \equiv$ the total number of non $\nu_{\mu}$ QE-like resonance production events for bin $i$

- $\left(\mathrm{N}_{D I S}^{b k g d}\right)_{i} \equiv$ the total number of non $\nu_{\mu}$ QE-like DIS plus non CC $\nu_{\mu}$ events for bin $i$

- $\left(\mathrm{w}_{\text {Total }}^{\text {bkgd }}\right)_{i} \equiv$ extracted scale factors for bin $i$, which describe how much to weight the total Monte Carlo background events, such that the Monte Carlo and data event rate are perfectly matched.

From the information given above, a scale factor for each component, Resonant and DIS plus others, is extracted per $\mathrm{Q}_{\mathrm{QE}, \text { proton }}^{2}$ bin $i$ using the linear relationship,

$$
\left(\left(w_{\text {Total }}^{\text {bkgd }}\right)_{i} \times \frac{\left(N_{\text {Total }}^{b k g d}\right)_{i}}{\left(N_{\text {Res }}^{b k g d}\right)_{i}}\right)_{s}=\left(w_{\text {Res }}^{b k g d}\right)_{i}+\left(w_{D I S}^{b k g d}\right)_{i} \times\left(\frac{\left(N_{D I S}^{b k g d}\right)_{i}}{\left(N_{\text {Res }}^{b k g d}\right)_{i}}\right)_{s},
$$


with $s=$ sideband $1,2,3,4$. The $\mathrm{y}$-intercept $\mathrm{w}_{\text {Res }}^{b k g d}$ and the slope $\mathrm{w}_{D I S}^{b k g d}$ are the extrapolated scale factors for bin $i$ for the two-component QE-like backgrounds. Figure 5.20 shows the linear fits that are obtained from the extrapolation technique.

The two-component QE-like background extrapolated scale factors are shown in Figure 5.21. Overall, the resonant events need to be scaled down by 50\%, while the DIS plus others should be scale up. This reduction that is observed for the resonant events is consistent with the extracted background scale factors found in the MINER $\nu \mathrm{A}$ pion production cross section analyses [89]. The DIS plus others scale factors increases for $\mathrm{Q}_{\mathrm{QE} \text {,proton }}^{2} \rightarrow 0$, which can suggests that the backgrounds in the low $\mathrm{Q}_{\mathrm{QE} \text {,proton }}^{2}$ regime consist of a significant contribution from the inelastic events in the region of $1.3<\mathrm{W}<1.6 \mathrm{GeV} / \mathrm{c}^{2}$.

The Monte Carlo predicted QE-like backgrounds (see Figure 5.22) are weighted by the extrapolated scale factors shown in Figure 5.21. The comparison between the data and absolutely normalized Monte Carlo after re-weighting the backgrounds (see Figure 5.22) shows that the Monte Carlo underestimates the data event rate. This could correspond to the lack of modeling of the multinucleon contribution to the $\nu_{\mu}$ QE-like cross section and a full description of the effects from FSIs.

The Monte Carlo constrained backgrounds are subtracted from the data, where the subtracted distribution is displayed in Figure 5.23. Both the shape and absolute error summaries for the background subtracted $\mathrm{Q}_{\mathrm{QE} \text {,proton }}^{2}$ distribution are shown in Figure 5.24, where an overview of the systematic uncertainties is summarized in Section 5.4. To obtain the uncertainty on the background subtracted data events, the following procedure is applied.

1. The Monte Carlo event rate for each of the various components which make-up the QE-like candidates (see Figure 5.15) and the sidebands (see Figure 5.18) are 

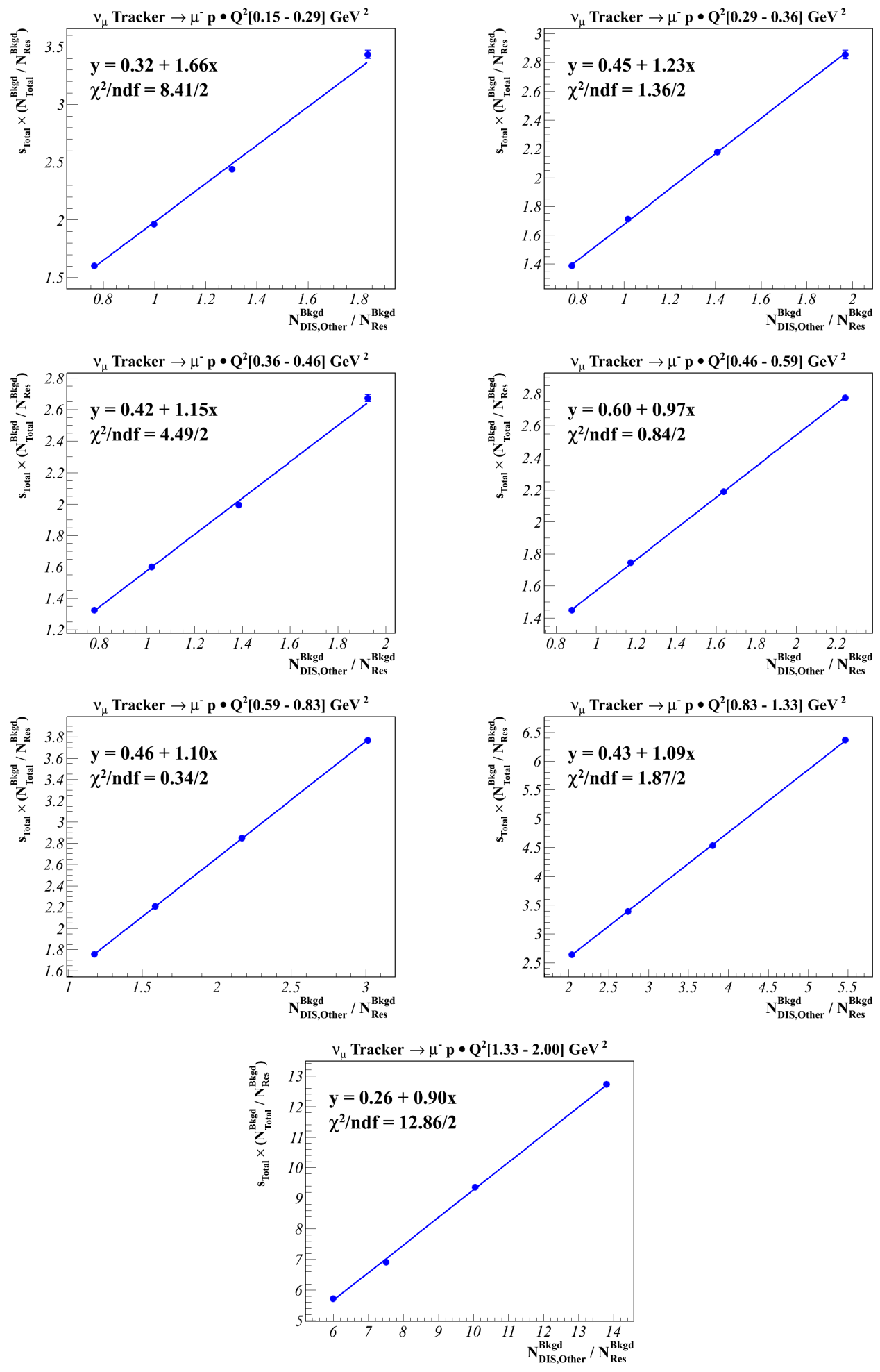

Figure 5.20. Linear fits for each $\mathrm{Q}_{\mathrm{QE} \text {,proton }}^{2}$ bin $i$, where the fit parameters give the scale factors for the two-component QE-like backgrounds. 


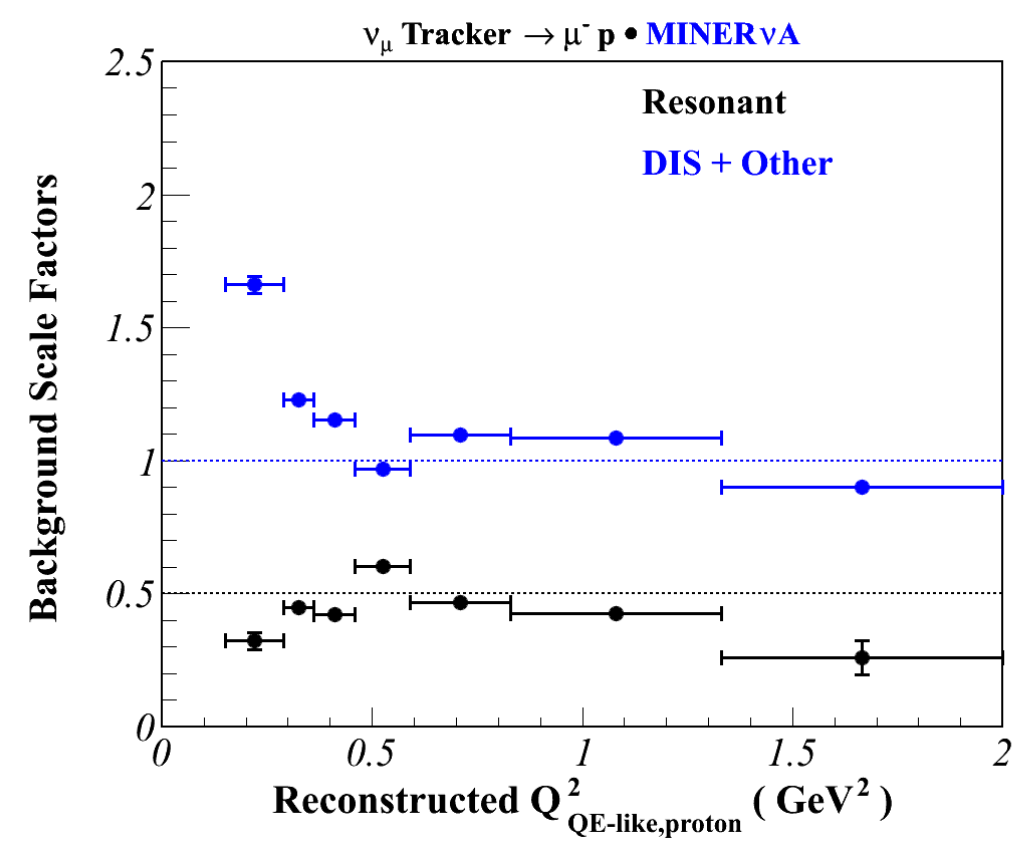

Figure 5.21. The scale factors for the two-component QE-like backgrounds.

predicted over and over again (commonly referred to the multi-universe procedure, which is described in Section 5.4) by simultaneously varying a parameter of a given systematic source which may contribute to the total uncertainty on the background subtraction procedure. Thus, each Monte Carlo variation $i$ predicts a differ event rate for the QE-like candidates $\mathrm{N}_{\text {source, } i}^{Q E-l i k e}$, which has a predicted background yield $\mathrm{N}_{\text {source }, i}^{\text {non } Q E-l i k e}$. In addition, the event rate for each component which make-up each of the sidebands has also varied.

2. The background procedure which is described above, is applied to each Monte Carlo universe. As a result, each Monte Carlo universe that gives an event rate $\mathrm{N}_{\text {source, } i}^{Q E-l i k e}$ with parameter $p$ varied for a given source $s$, has it's own set of the two-component extrapolated scale factors.

3. Next, the two-component backgrounds for each of the QE-like variations are reweighted using it's own set of two-component extrapolated scale factors. The 

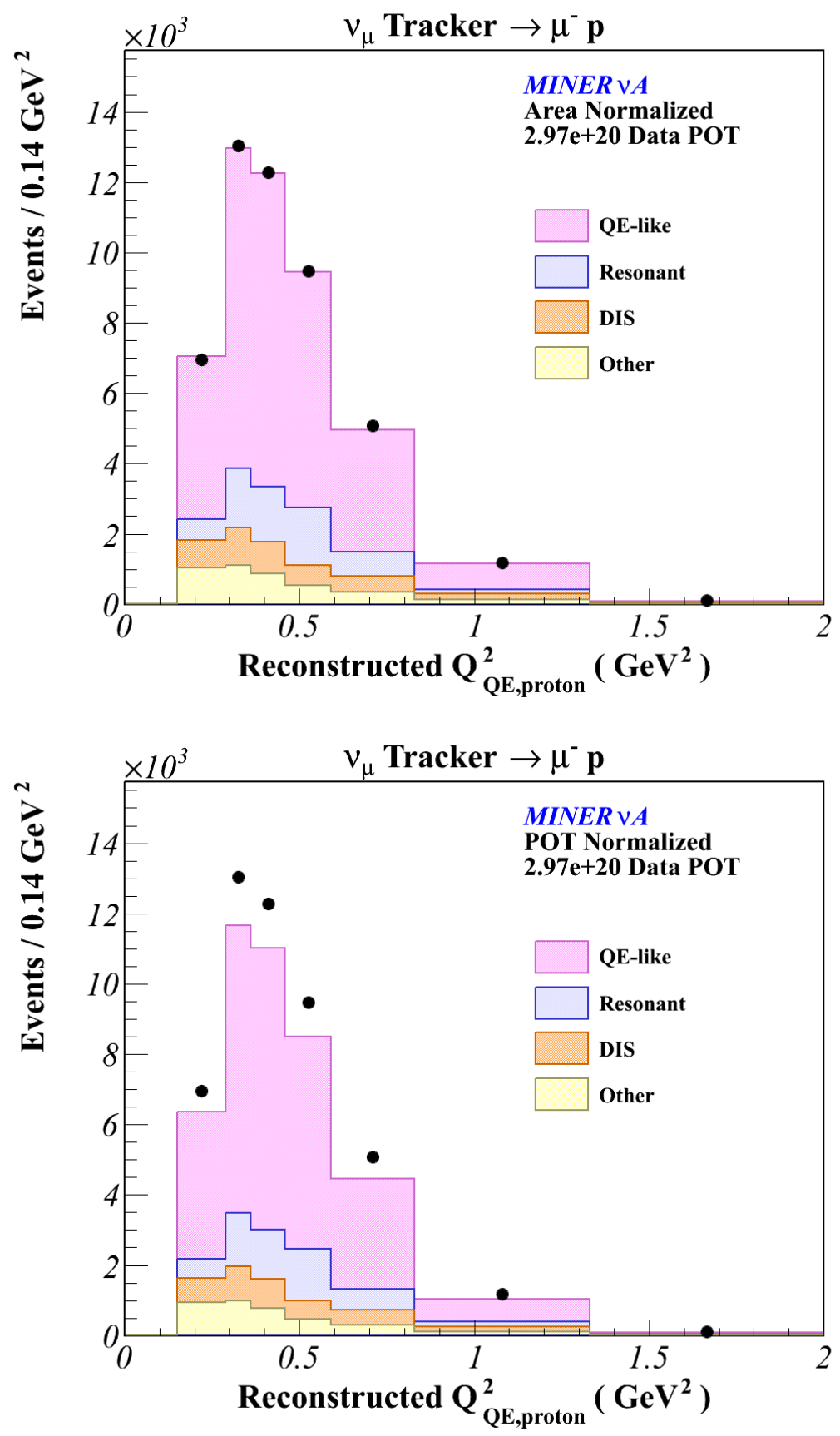

Figure 5.22. The area (top) and absolutely (bottom) normalized data and Monte Carlo comparison for the QE-like candidates with the constrained backgrounds. For the absolutely normalized distribution, an enhancement in the data is observed. 

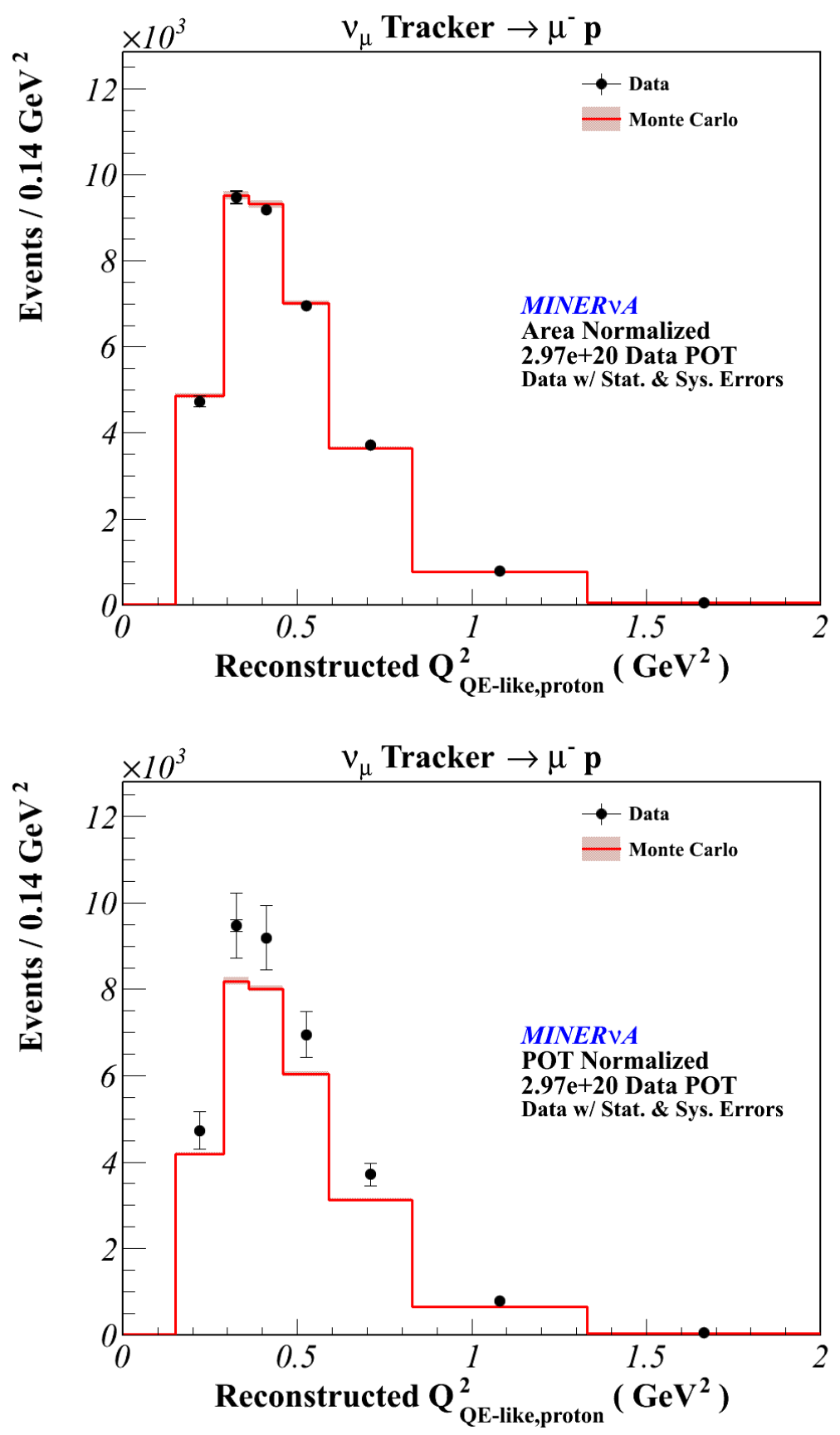

Figure 5.23. The area (top) and absolutely (bottom) normalized data and Monte Carlo comparison for the background subtracted distribution. The Monte Carlo only has statistical error bars. 
nominal Monte Carlo and the Monte Carlo universes constrained backgrounds are given by,

$$
\mathbf{N}^{\text {nonQE-like }}=\left(\begin{array}{c}
N_{0}^{\text {nonQE-like }} \\
N_{1}^{\text {nonQE-like }} \\
\cdot \\
N^{\text {nonQE-like }} \\
\cdot \\
N_{n}^{\text {nonQE-like }}
\end{array}\right),
$$

where the $n$ entries correspond to each universe in parameter space and $\mathrm{N}^{\text {non } Q E-l i k e}$ is the yield for the nominal Monte Carlo constrained backgrounds.

4. Each background variation is subtracted from the data,

$$
\mathbf{N}_{\text {signal }}^{\text {data }}=\left(\begin{array}{c}
N^{\text {data }}-N_{0}^{\text {nonQE-like }} \\
N^{\text {data }}-N_{1}^{\text {nonQE-like }} \\
\cdot \\
N^{\text {data }}-N^{\text {nonQE-like }} \\
\cdot \\
N^{\text {data }}-N_{n}^{\text {non } Q E-l i k e}
\end{array}\right),
$$

which gives different predictions of the event rate for background subtracted data.

5. The last step is to calculate the systematic error for a given source with parameter $p$ varied using a covariance matrix, which is described in Section 5.4. Note that the parameter $p$ is usually varied within $\pm 1 \sigma$ of it's uncertainty.

The largest contributions to the background subtracted data are due to GENIE modeling of the neutrino interactions (the cross section models) and the hadrons trans- 

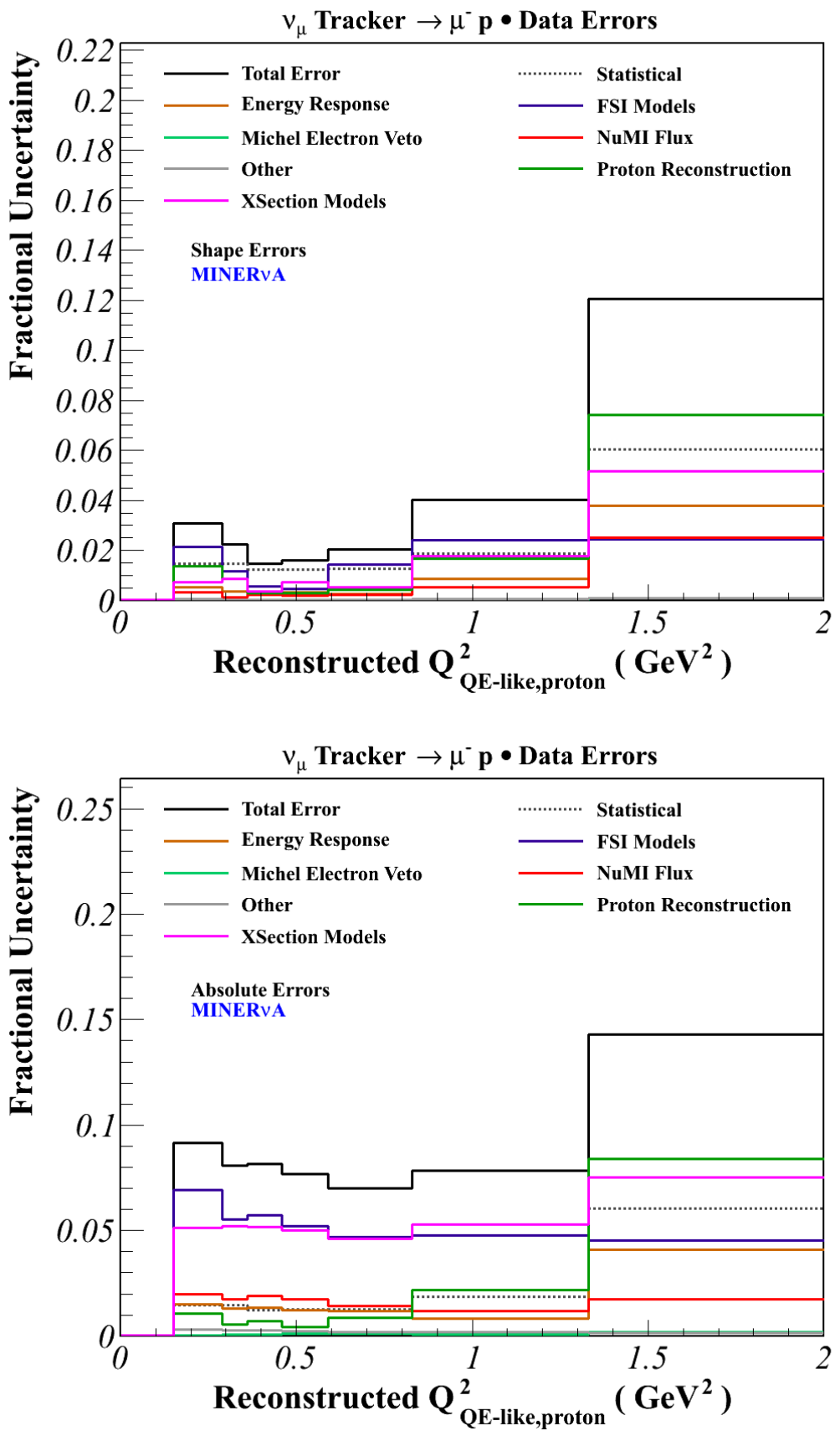

Figure 5.24. The shape (top) and absolute (bottom) error summary for the background subtracted distribution. The flux does not contribute to the systematic uncertainty on the background subtraction procedure. 
portation in the nucleus (the final state intra-nuclear cascade models). In addition, the uncertainty is the largest for the highest $\mathrm{Q}_{\mathrm{QE} \text {,proton }}^{2}$ bin, which is due to calculating the systematic uncertainty with a smaller number of events.

\subsubsection{BIN MIGRATION AND UNFOLDING}

The reconstruction of kinematic observables, such as a particle scattering angle or initial energy in the final state, is not exact due to the finite detector resolution. The detector smearing causes events to migrate from bin to bin, and often in one direction. The background subtracted data is unfolded using the inverse of a smearing matrix (commonly referred to as the unfolding matrix), that is completely determined from the Monte Carlo truth information and is given by,

$$
U_{i j}=\frac{N_{\text {true }, j}^{r e c, i}}{N_{\text {true }, j}^{r e c o}},
$$

where $\mathrm{N}_{t r u e, j}^{r e c o}$ is the number of reconstructed signal events generated in bin $j$ and $\mathrm{N}_{\text {true }, j}^{\text {reco }, i}$ is a subset of those events which are reconstructed in bin $i$. The smearing matrix is inverted and utilized to unfold the data, via

$$
N_{\text {data, } i}^{\text {true }}=\sum_{j} N_{\text {data,j }}^{r e c o} U_{i j}^{-1}
$$

The unfolding matrix converts the reconstructed number of events in the bin $j$ to the true number of events in the bin $i$. There exists various unfolding techniques, which have been studied by the previous MINER $\nu \mathrm{A}$ QE analyses. Based on the QE analyses conclusions, the iterative Bayesian procedure using four iterations is utilized to unfold the reconstructed distribution.[90]. The unfolding matrix that is utilized for this analysis is presented in Figure 5.25

The reconstructed $\mathrm{Q}_{\mathrm{QE} \text {,proton }}^{2}$ distribution is unfolded to a $\mathrm{Q}_{\mathrm{QE} \text {,proton }}^{2}$ calculation using Equation 5.2.1, where the kinetic energy is taken from the most energetic proton 


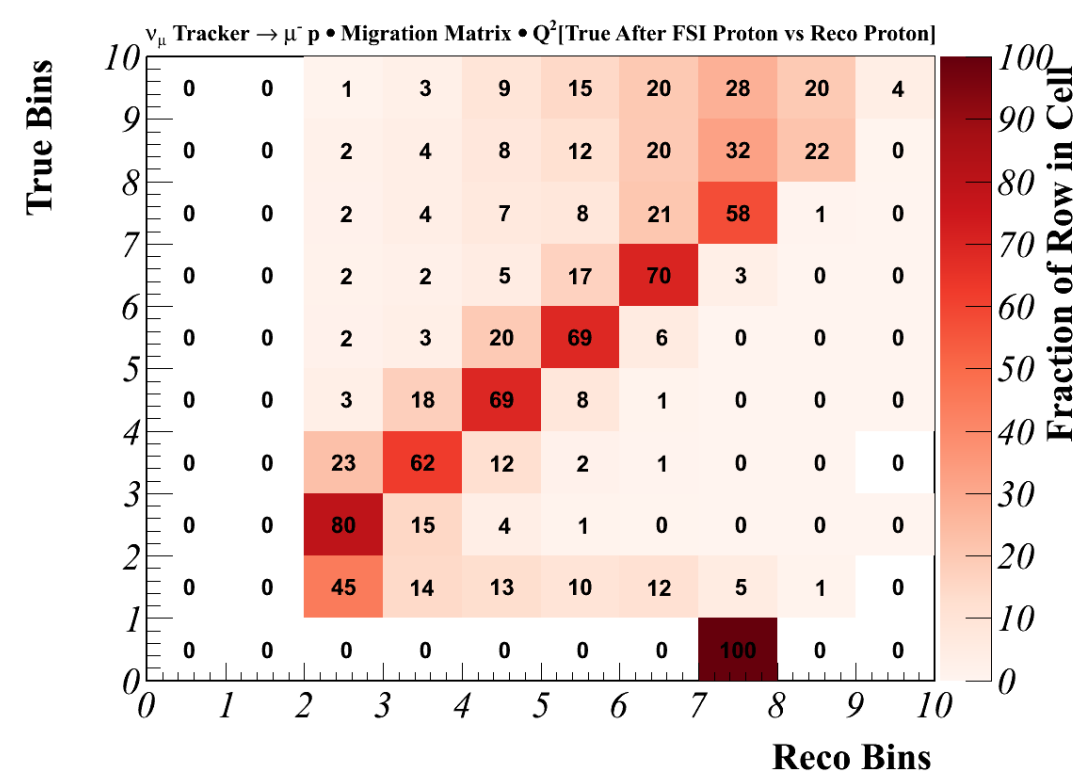

Figure 5.25. Plotted is the smearing matrix, where the inverse of this matrix is defined as the unfolding matrix. The event in the underflow bin corresponds to neutrino interactions that have a proton with no kinetic energy in the final state, but a high energy neutron. The neutron immediately re-scatters in the detector and knocks out a proton that is tracked.

in the final state. Therefore, the unfolding only accounts for the most energetic proton propagation through the MINER $\nu \mathrm{A}$ detector. Thus, the application of the unfolding technique does not depend on the GENIE modeling of the final state interactions. Note that this $\mathrm{Q}_{\mathrm{QE} \text {,proton }}^{2}$ calculation is not the same as the true $\mathrm{Q}^{2}$ at the scattering vertex.

The results of the unfolding are presented in Figure 5.26. In general, the momentum is reconstructed too low for the higher energy protons due to the nuclear re-scattering in the detector. Therefore, the unfolding matrix moves events toward the high $\mathrm{Q}_{\mathrm{QE} \text {,proton }}^{2}$ bins and the spectrum broadens. Note that the unfolding procedure moves events to the first $\mathrm{Q}_{\mathrm{QE} \text {,proton }}^{2}$ bin, which was unoccupied originally and is below the tracking threshold. The smearing matrix in Figure 5.25 shows that events with a leading proton less than the tracking threshold are momentum reconstructed too high. This is due to the inefficiency of the reconstruction. The track cleaning 

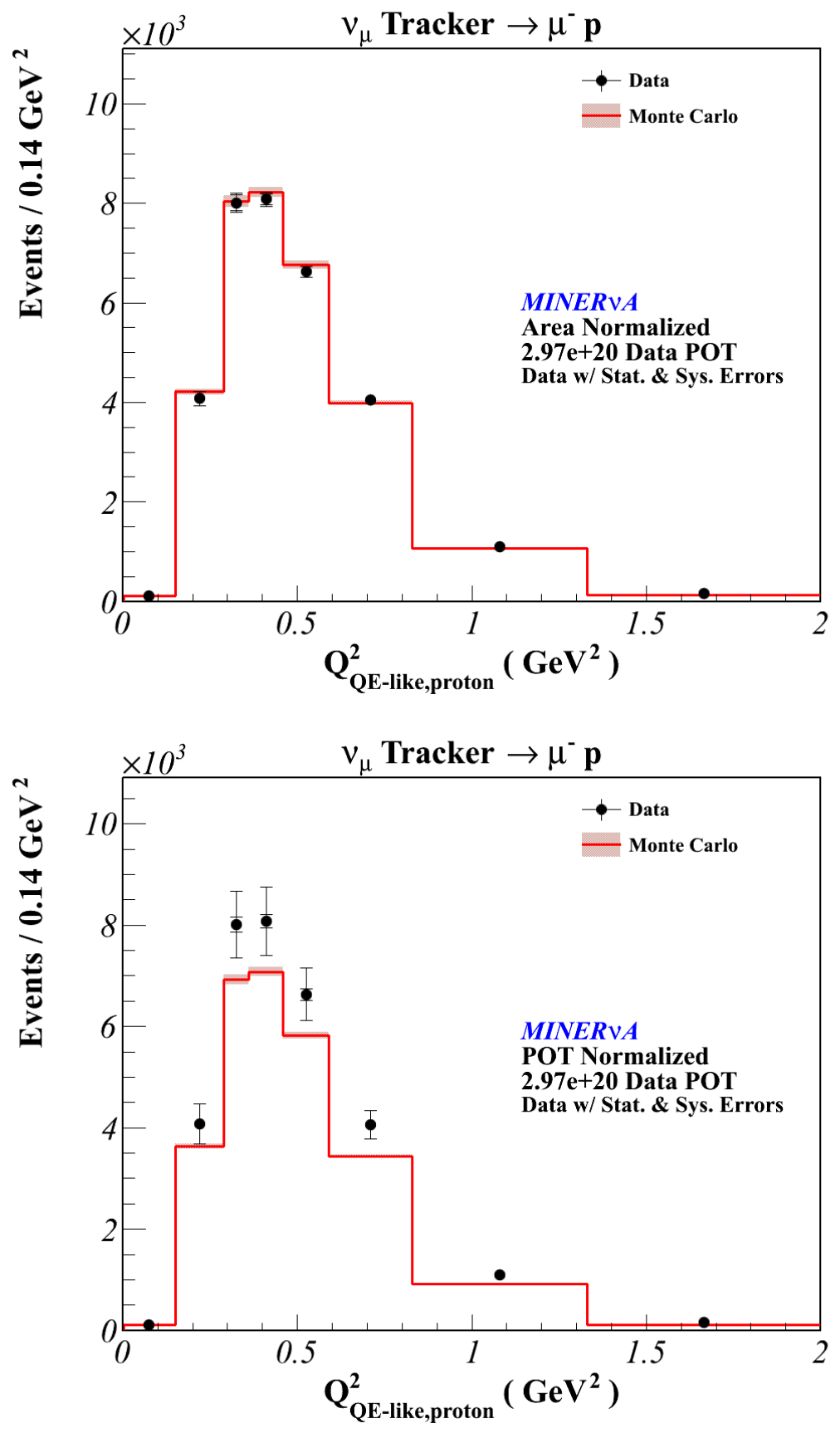

Figure 5.26. The area (top) and absolutely (bottom) normalized data and Monte Carlo comparison for the unfolded distribution. 
procedure removes too much muon energy from the muon track, which is consumed by the short tracking algorithms which create a proton track out of a combination of the proton and muon energies. These events usually resemble an event topology where the muon and proton overlap in detector space. Recall that the proton identification consistency check is relax cut, thus these events can pass the pID score cut. The vertex $\mathrm{Z}$ position is more likely to be reconstructed too far upstream relative to the true vertex $\mathrm{Z}$ position for these events. Again, this analysis accounts for the energy loss in the materials between the track's origin and the event vertex. Therefore, the momentum is reconstructed too high for these protons. An event scan of the $\nu_{\mu}$ QE-like candidates in both the data and Monte Carlo will characterized if this feature is significant for this analysis.

Both the shape and absolute error summaries for the unfolding procedure is summarized in Figure 5.27. The application of the unfolding is not affected by the uncertainty on the GENIE primary interaction cross section models and FSI intranuke cascade models, because the smearing matrix is designed to be as model-independent as possible.

\subsubsection{EFFICIENCY AND ACCEPTANCE CORRECTIONS}

As mentioned previously, due to the detector and reconstruction inefficiencies, not all of the observed $\nu_{\mu}$ QE-like events enter into the final event selection. As shown in Table 5.1, the number of tracks condition contributes the most to the loss in the event rate. The proton pID consistency check also contributes significantly to the decline in efficiency. The efficiency for selecting the $\nu_{\mu}$ QE-like events per bin $i$ is determined from the Monte Carlo and is given by,

$$
\epsilon_{i}=\left(\frac{N_{g e n}^{\text {pass }}}{N_{\text {gen }, \text { fid }}}\right)_{i}
$$



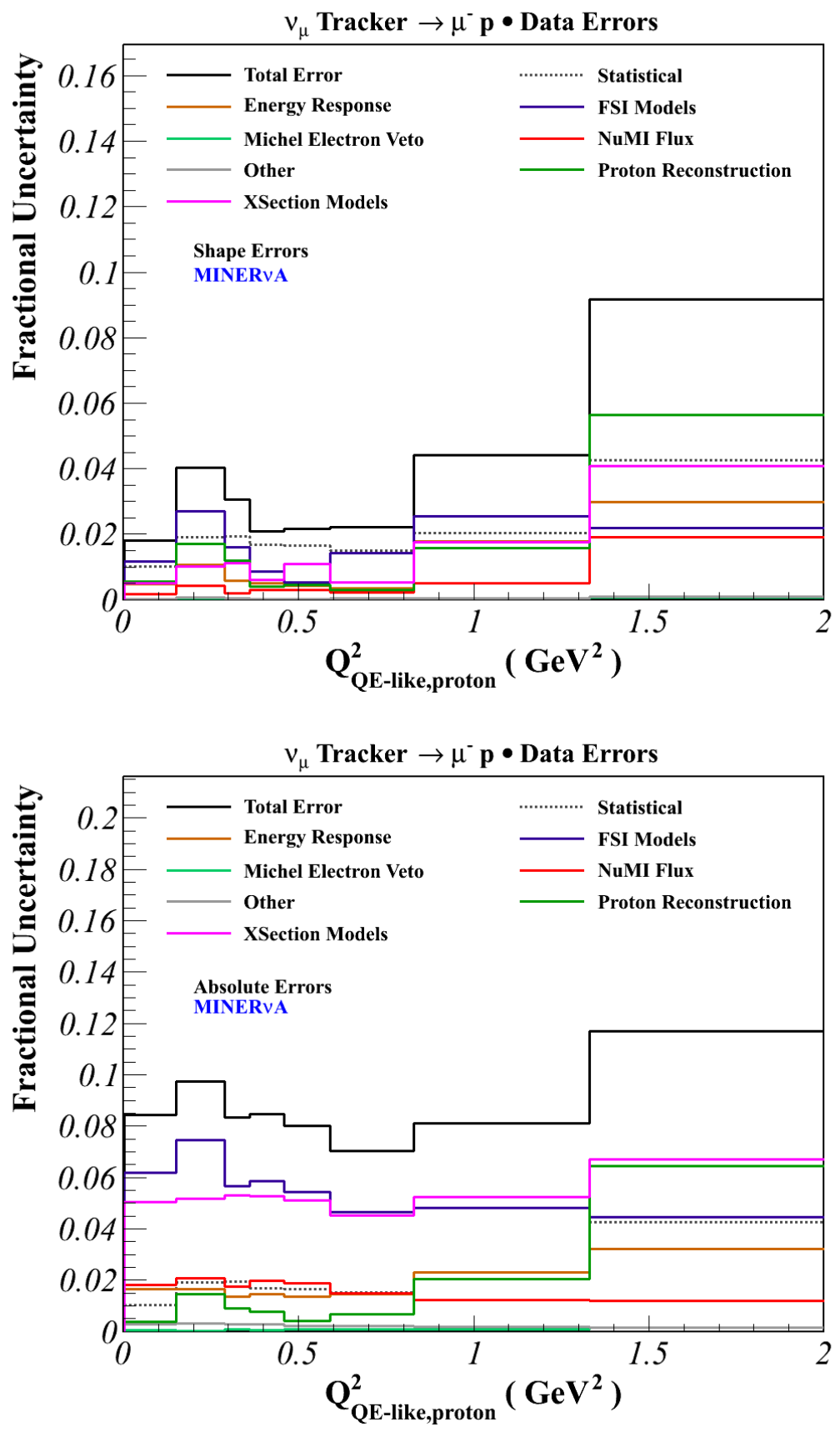

Figure 5.27. The shape (top) and absolute (bottom) error summaries for the unfolding procedure. 
where $\mathrm{N}_{g e n, f i d}$ is the number of signal events generated in the fiducial volume for bin $i$ and $\mathrm{N}_{\text {gen }}^{\text {pass }}$ is the number of generated signal events that appear in the signal selection for bin $i$.

The reconstruction performance may vary slightly between the data and Monte Carlo events. Therefore, the efficiency function which is calculated using Equation C.0.1 for the final event selection may differ between the Monte Carlo and the data. The data and Monte Carlo efficiency discrepancy is a result of the mis-modeling of the detector inefficiencies or external activity that may enter the detector. There are two components from the reconstruction that may produce a discrepancy for the efficiency between the data and simulated events;

- The number of tracks which addresses the efficiency for tracking protons in the detector, and

- The proton pID performance which accounts for the mis-modeling of the proton propagation in the detector.

Currently, the analysis presented in this dissertation does not produce any correction factors to account for reconstruction inefficiencies that may be observed between the data and Monte Carlo based on the sources describe above. This also implies that a systematic uncertainty which accounts for the differences between the data and Monte Carlo for reconstructing hadron tracks and identifying the proton, is not evaluated. In the very near term future, the track efficiency will be estimated from scanning events in both the Monte Carlo and data. In addition, the proton pID efficiency will be determined from the data, which is obtained from a beam of protons propagating through a detector that is a smaller version of the MINER $\nu$ A detector.

The efficiency function and the systematic uncertainty that are presented in Figure 5.28 is model-dependent and is used to correct the data. Figure 5.29 shows the distribution after correcting for the efficiency, where both the shape and absolute error summaries are presented in Figure 5.30. The primary contributors to the systematic 

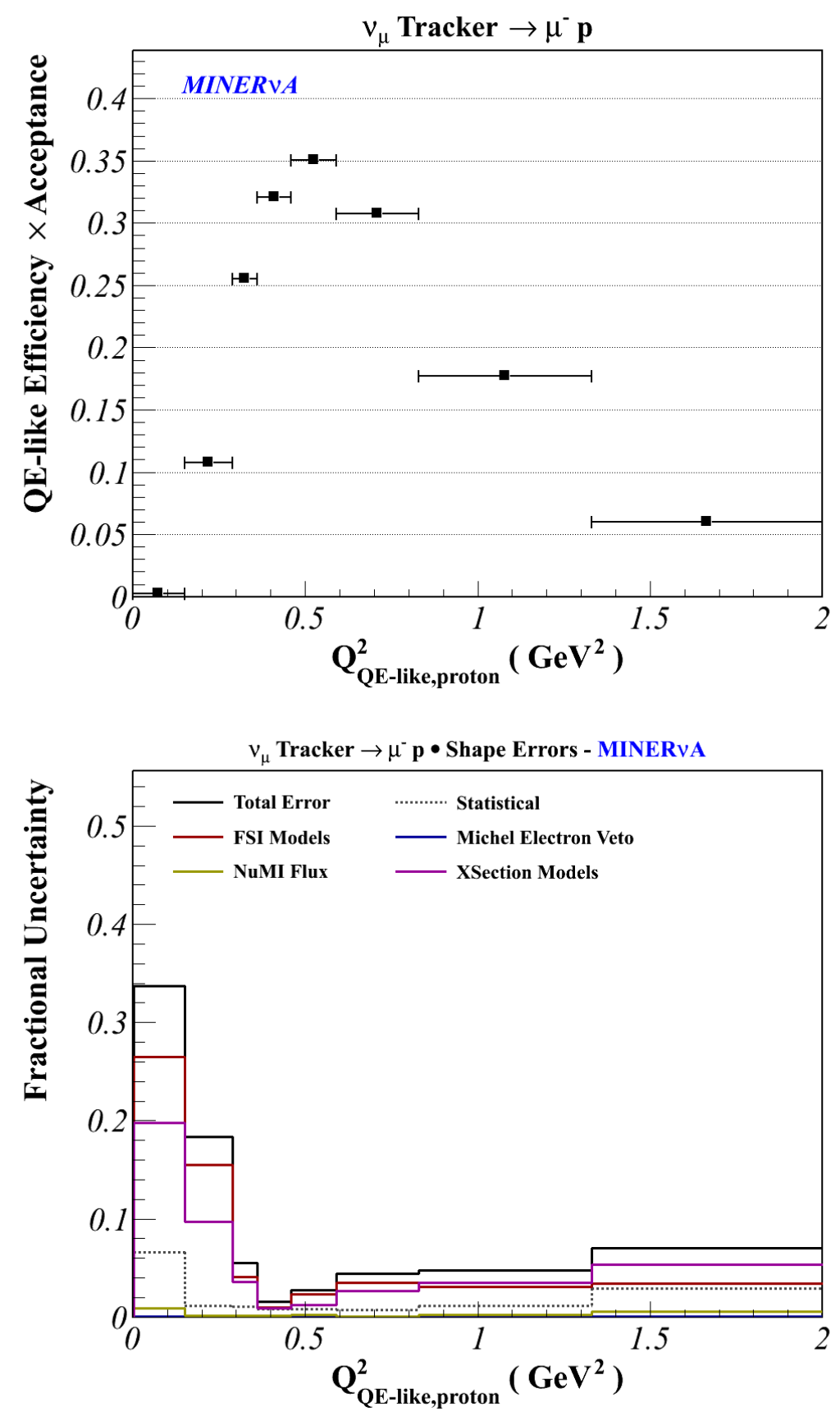

Figure 5.28. (Top) Product of the Monte Carlo efficiency and acceptance for reconstructing the $\nu_{\mu}$ QE-like events and the (bottom) absolute systematic uncertainty on the efficiency function. Recall that the tracking threshold is responsible for the loss in efficiency for the low $\mathrm{Q}_{\mathrm{QE} \text {,proton }}^{2}$ bin, while the pID criteria and detector acceptance dominate in the high $\mathrm{Q}_{\mathrm{QE} \text {,proton }}^{2}$ bin. 
uncertainty is GENIE modeling of the neutrino interactions and hadron transportation in the nucleus. The GENIE sources that give rise to the large errors are the following:

- The resonance production cross section free parameter, $\mathrm{M}_{A}$.

- The INC modeling of the pion absorption and inelastic cross sections.

The fraction of pions that are produced directly effects the efficiency of the QE-like signal. For the QE-like events that are not quasielastic, the proton kinematics are correlated with the pion kinematics.

\subsubsection{DIFFERENTIAL CROSS-SECTION RESULTS}

The final step is to divide the efficiency-corrected distribution by the integrated flux and bin width, then normalize the results by the number of target nucleons to obtain the $\mathrm{d} \sigma / \mathrm{dQ}_{\mathrm{QE}-\text { like,proton }}^{2}$ as a function of $\mathrm{Q}_{\mathrm{QE}-\text { like,proton }}^{2}$. The neutrino flux is integrated over the entire spectrum, $0<\mathrm{E}_{\nu}<100 \mathrm{GeV}$, where the total flux is estimated as $\Phi=3.286 \times 10^{-8} / \mathrm{cm}^{2} / \mathrm{POT}$. The number of target nucleons is $\mathrm{T}=9.771 \times 10^{50}$, where the calculation is based on the total number of nucleons within the MINER $\nu \mathrm{A}$ standard fiducial volume [91]. Figure 5.31 shows the differential cross section results for the $\nu_{\mu} \mathrm{QE}$-like sample. The results show that the Monte Carlo underestimates the event rate for the $\nu_{\mu}$ QE-like channel, but are within the systematic uncertainties. Tables 5.3.4 and 5.3.4 display the cross section measurement for each bin and the fractional systematic uncertainties on the $\mathrm{d} \sigma / \mathrm{dQ}_{\mathrm{QE}-\text { like,proton }}^{2}$, respectively. Both the shape and absolute error summaries for the cross section are shown in Figure 5.32, where an overview of each systematic uncertainty is summarized in the following section.

\section{The Cross Section Measurement in the First $Q_{Q E \text {,proton }}^{2}$ Bin}

Before interpreting the cross section result, the measurement in the first $\mathrm{Q}_{\mathrm{QE} \text {,proton }}^{2}$ bin is discussed. Recall that due to several reconstruction inefficiencies that are 

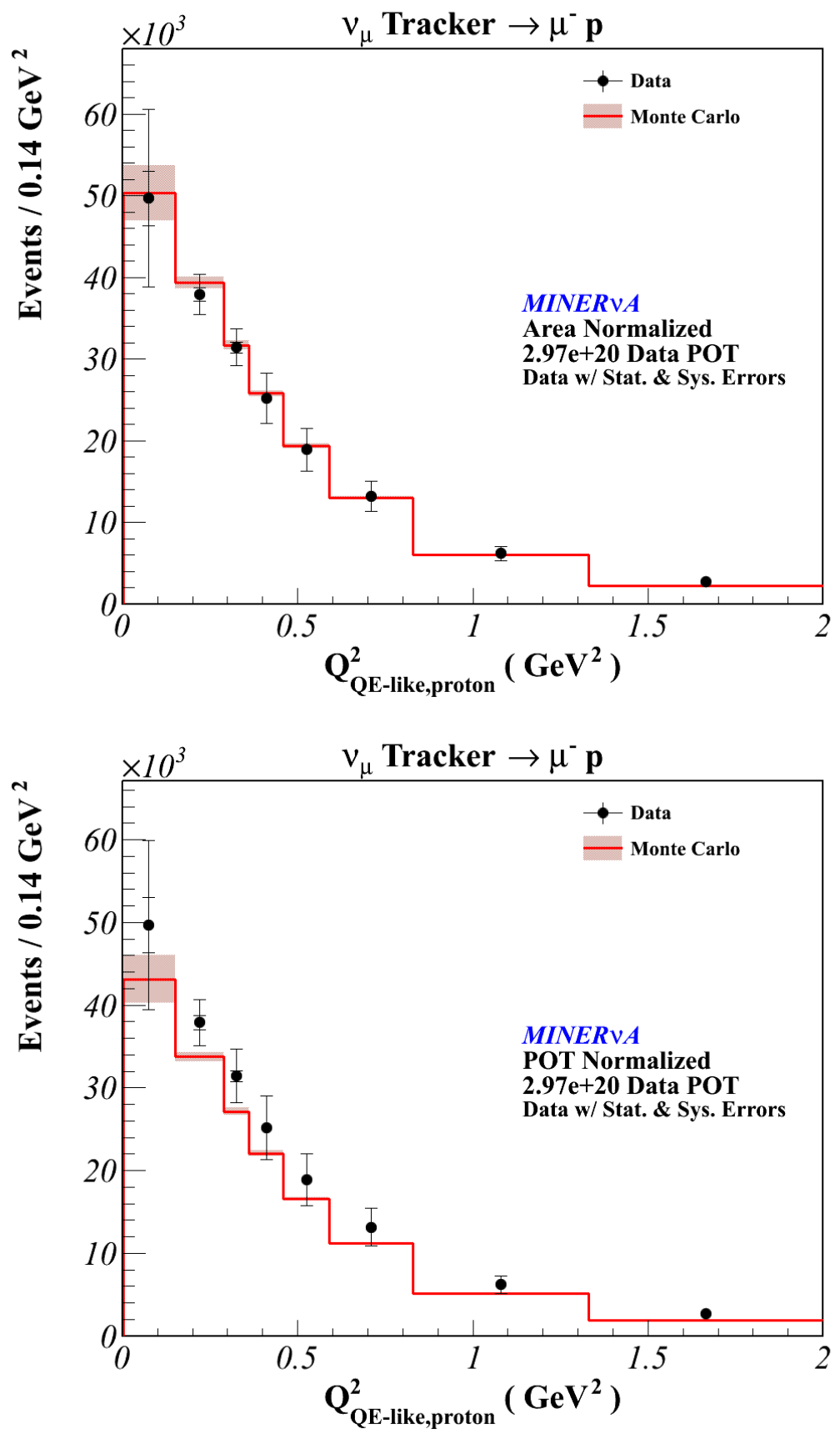

Figure 5.29. The efficiency-corrected area (top) and absolutely (bottom) normalized distributions. 

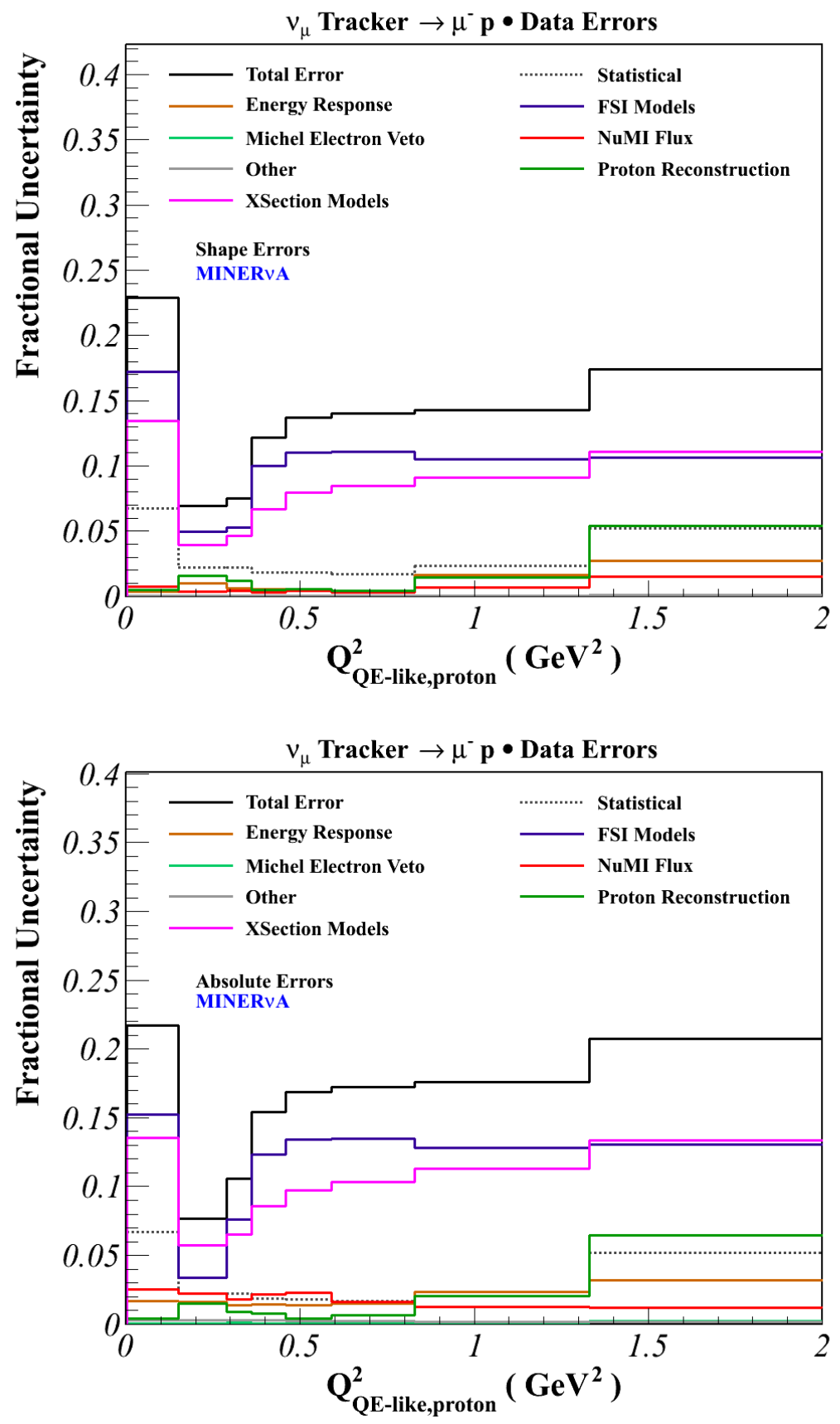

Figure 5.30. Plotted are both the shape (top) and absolute (bottom) error summaries for the efficiency-corrected distribution. 

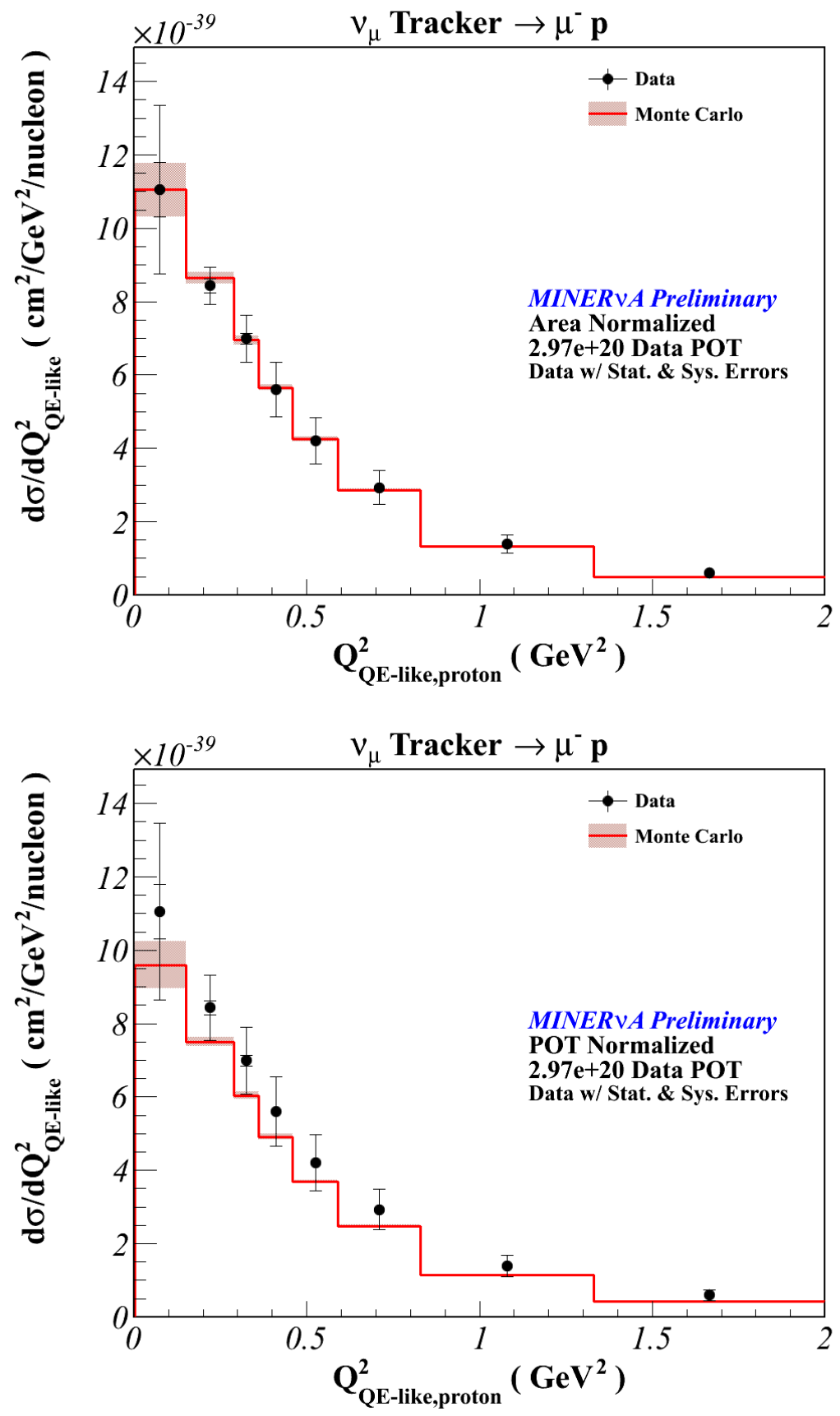

Figure 5.31. The area (top) and absolutely (bottom) normalized data and Monte Carlo comparison of the cross sections for the QE-like channel. 

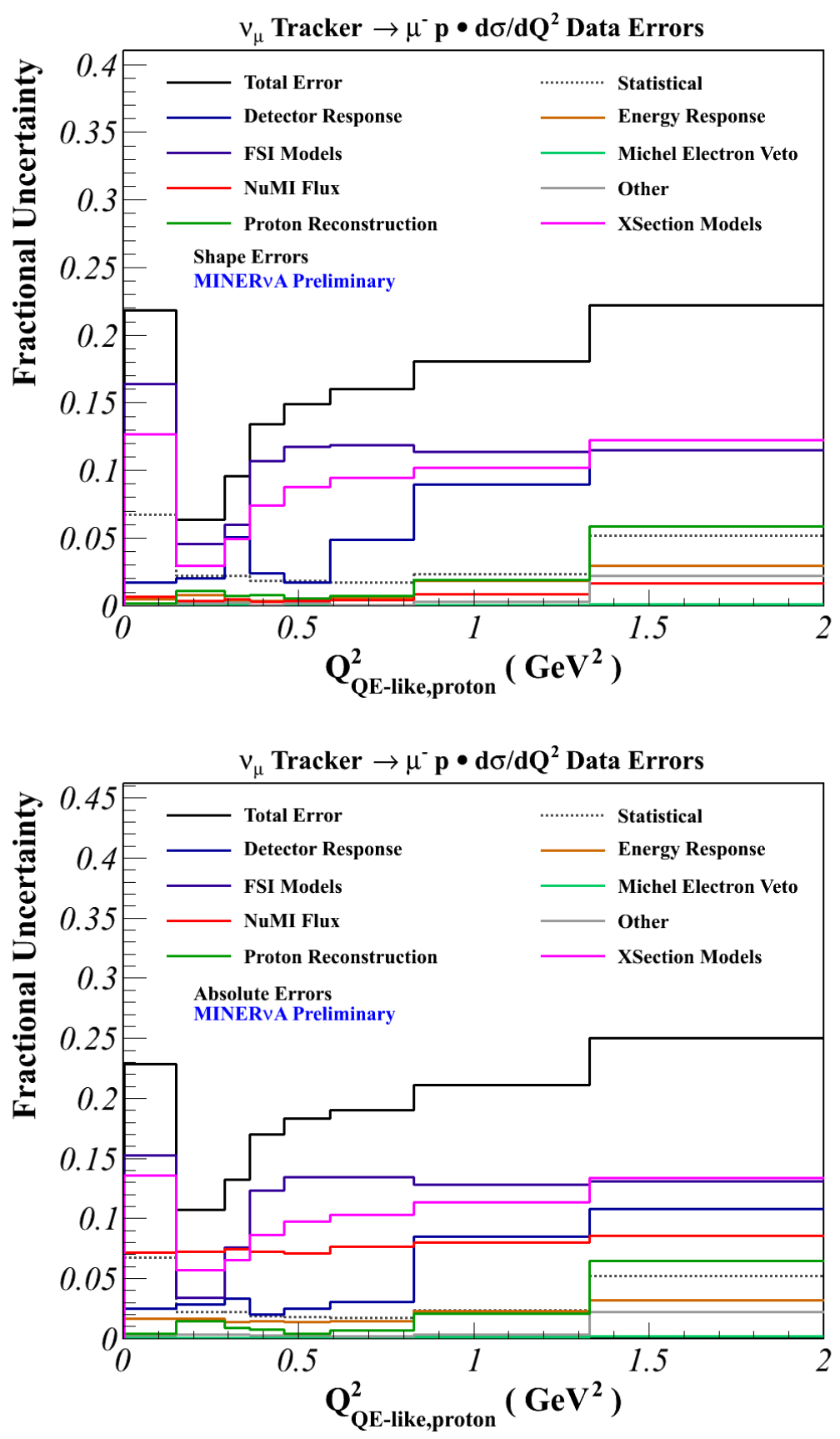

Figure 5.32. The shape (top) and absolute (bottom) error summaries of the differential cross section. Notice for the absolute errors, the uncertainty on the flux becomes significant. 
Table 5.4. Flux-integrated $\mathrm{d} \sigma / \mathrm{dQ}_{\mathrm{QE}-\text { like,proton }}^{2}$ Summary. Both the statistical (first) and absolute systematic (second) errors are presented for each measurement.

\begin{tabular}{c||c}
\hline \hline $\begin{array}{c}\mathrm{Q}_{\mathrm{QE} \text {,proton }}^{2} \\
\left(\mathrm{GeV}^{2}\right)\end{array}$ & $\begin{array}{c}\text { Cross-section } \\
\left(10^{-38} \mathrm{~cm}^{2} / \mathrm{GeV}^{2} / \text { nucleon }\right)\end{array}$ \\
\hline $0.0-0.15$ & $1.106 \pm 0.074 \pm 0.241$ \\
$0.15-0.29$ & $0.843 \pm 0.019 \pm 0.088$ \\
$0.29-0.36$ & $0.699 \pm 0.015 \pm 0.091$ \\
$0.36-0.46$ & $0.561 \pm 0.010 \pm 0.095$ \\
$0.46-0.59$ & $0.420 \pm 0.008 \pm 0.077$ \\
$0.59-0.83$ & $0.293 \pm 0.005 \pm 0.055$ \\
$0.83-1.33$ & $0.138 \pm 0.003 \pm 0.028$ \\
$1.33-2.0$ & $0.060 \pm 0.003 \pm 0.015$ \\
\hline
\end{tabular}

Table 5.5. Fractional systematic uncertainties on the $\mathrm{d} \sigma / \mathrm{dQ}_{\mathrm{QE}-\text { like,proton measurement for }}^{2}$ each $\mathrm{Q}_{\mathrm{QE} \text {,proton }}^{2}$ bin. Both the absolute (top) and area (bottom) errors are presented for each $\mathrm{Q}_{\mathrm{QE}}^{2}$,proton bin and the contributors are (I) flux, (II) neutrino interaction models, (III) final state interaction models, (IV) detector response, (V) energy response, (VI) proton reconstruction, (VII) Michel electron veto, and (VIII) other

\begin{tabular}{|c||cccccccc|}
\hline \hline \multicolumn{10}{|c|}{ Absolute Errors } \\
\hline $\mathrm{Q}_{\mathrm{QE} \text {,proton }}^{2}\left(\mathrm{GeV}^{2}\right)$ & I & II & III & IV & V & VI & VII & VIII \\
\hline $0.0-0.15$ & 0.072 & 0.135 & 0.152 & 0.025 & 0.017 & 0.004 & 0. & 0.003 \\
$0.15-0.29$ & 0.073 & 0.057 & 0.034 & 0.029 & 0.016 & 0.015 & 0. & 0.003 \\
$0.29-0.36$ & 0.075 & 0.065 & 0.076 & 0.033 & 0.014 & 0.009 & 0.001 & 0.004 \\
$0.36-0.46$ & 0.073 & 0.086 & 0.123 & 0.020 & 0.015 & 0.007 & 0.001 & 0.003 \\
$0.46-0.59$ & 0.071 & 0.097 & 0.134 & 0.025 & 0.014 & 0.004 & 0.001 & 0.002 \\
$0.59-0.83$ & 0.076 & 0.103 & 0.135 & 0.030 & 0.023 & 0.007 & 0.001 & 0.002 \\
$0.83-1.33$ & 0.080 & 0.113 & 0.128 & 0.085 & 0.032 & 0.021 & 0.001 & 0.003 \\
$1.33-2.0$ & 0.086 & 0.134 & 0.131 & 0.108 & 0.081 & 0.064 & 0.002 & 0.033 \\
\hline \multicolumn{10}{|c}{ Shape Errors } \\
\hline $0.0-0.15$ & 0.007 & 0.126 & 0.164 & 0.017 & 0.005 & 0.002 & 0. & 0.001 \\
$0.15-0.29$ & 0.003 & 0.029 & 0.046 & 0.020 & 0.008 & 0.011 & 0. & 0.001 \\
$0.29-0.36$ & 0.004 & 0.049 & 0.060 & 0.051 & 0.004 & 0.007 & 0. & 0.002 \\
$0.36-0.46$ & 0.003 & 0.074 & 0.107 & 0.024 & 0.003 & 0.008 & 0. & 0.003 \\
$0.46-0.59$ & 0.004 & 0.088 & 0.117 & 0.017 & 0.005 & 0.006 & 0. & 0.001 \\
$0.59-0.83$ & 0.004 & 0.094 & 0.118 & 0.049 & 0.005 & 0.007 & 0. & 0.001 \\
$0.83-1.33$ & 0.008 & 0.102 & 0.114 & 0.089 & 0.019 & 0.019 & 0. & 0.002 \\
$1.33-2.0$ & 0.016 & 0.122 & 0.115 & 0.115 & 0.030 & 0.059 & 0. & 0.032 \\
\hline
\end{tabular}


explained in Subsection 5.3.2, events where the leading proton has a momentum less than $450 \mathrm{MeV} / \mathrm{c}$ can enter into the $\nu_{\mu}$ QE-like event selection. As expected, the unfolding matrix moves events into the bin below the tracking threshold (the first $\mathrm{Q}_{\mathrm{QE} \text {,proton }}^{2}$ bin). Thus, the efficiency function (see Figure 5.28) corrects for the event rate in the first $\mathrm{Q}_{\mathrm{QE} \text {,proton }}^{2}$ bin with an efficiency value less than $1 \%$.

As indicated in Figure 5.31, the cross section measurement for the first $\mathrm{Q}_{\mathrm{QE} \text {,proton }}^{2}$ bin produces the largest area and error. This measurement completely drives the calculation of the shape errors, where the technique is discussed in Section 5.4. As a result, the total absolute and shape systematic errors are nearly identical, as shown in Figure 5.32. The measurement which is produced in this bin is a fabrication of the unfolding technique and untrustworthy. Since the tracking threshold prevents the detection of $\nu_{\mu}$ QE-like events with $\mathrm{Q}_{\mathrm{QE} \text {,proton }}^{2}<0.15 \mathrm{GeV}^{2}$, a simple solution for handling the first $\mathrm{Q}_{\mathrm{QE} \text {,proton }}^{2}$ bin is to alter the signal definition to reflect the tracking threshold. Therefore, the cross section is measured in a kinematic phase space where the events are reconstructed. The $\nu_{\mu}$ QE-like signal is redefined as,

- one muon

- no mesons

- any number of protons with at least one proton above tracking threshold $\left(\mathrm{p}_{\text {proton }}>\right.$ $450 \mathrm{MeV} / \mathrm{c})$.

The cross section measurement produce using the $\nu_{\mu}$ QE-like signal with the condition on the proton's energy is shown in Figure 5.33. Both the absolute and shape systematic error summaries are given in Figure 5.34. The fractional uncertainties on the systematic shape errors for GENIE modeling of the neutrino cross sections and hadron transportation in the nucleus are reduced, as indicated in Table 5.3.4. The systematic uncertainties on the GENIE models are better controlled.

Table 5.3.4 shows the comparison between the cross section measurements with and without the condition on the proton's momentum. There is an $\sim 30 \%$ reduction 

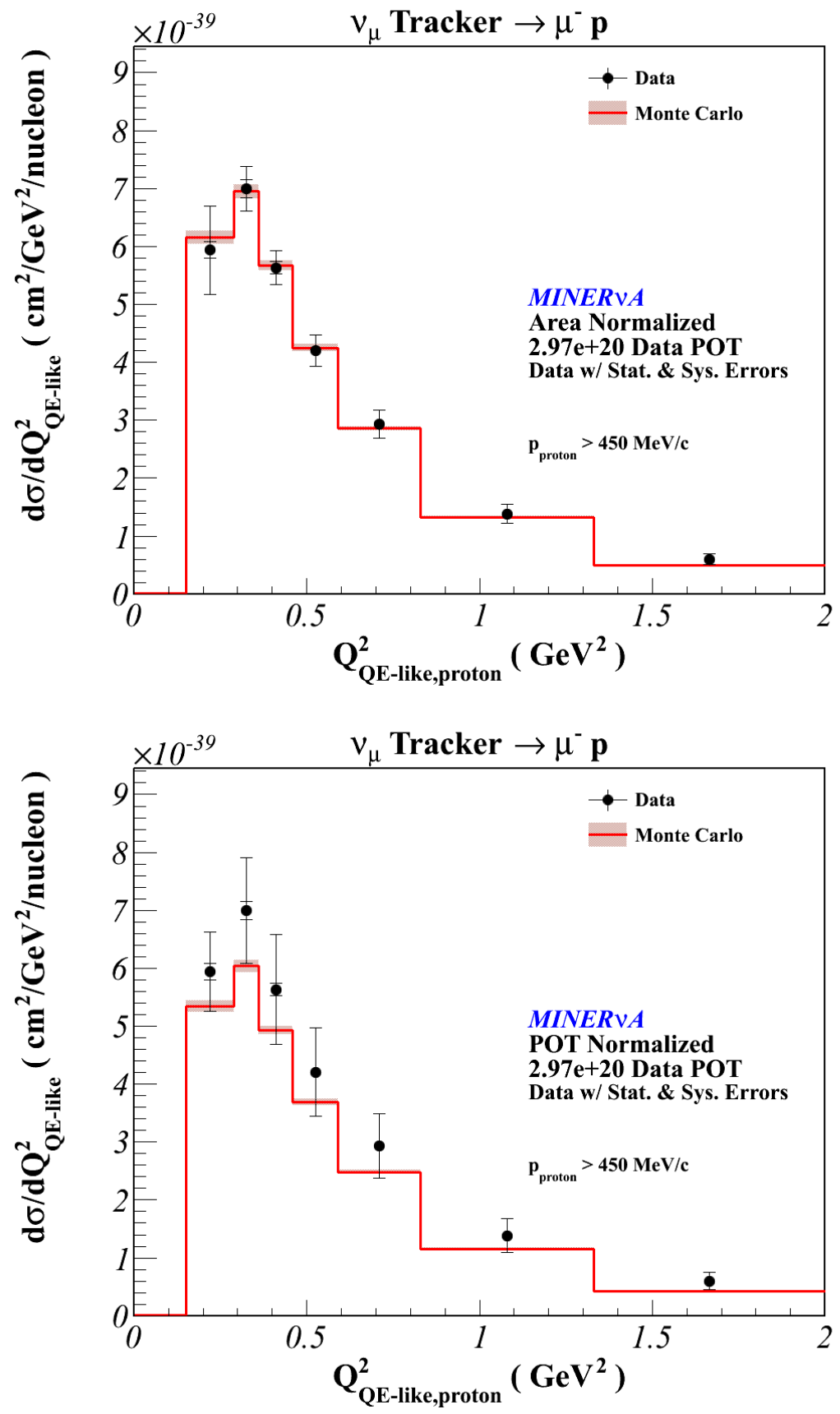

Figure 5.33. The area (top) and absolutely (bottom) normalized data and Monte Carlo comparison of the cross sections for the QE-like channel. 

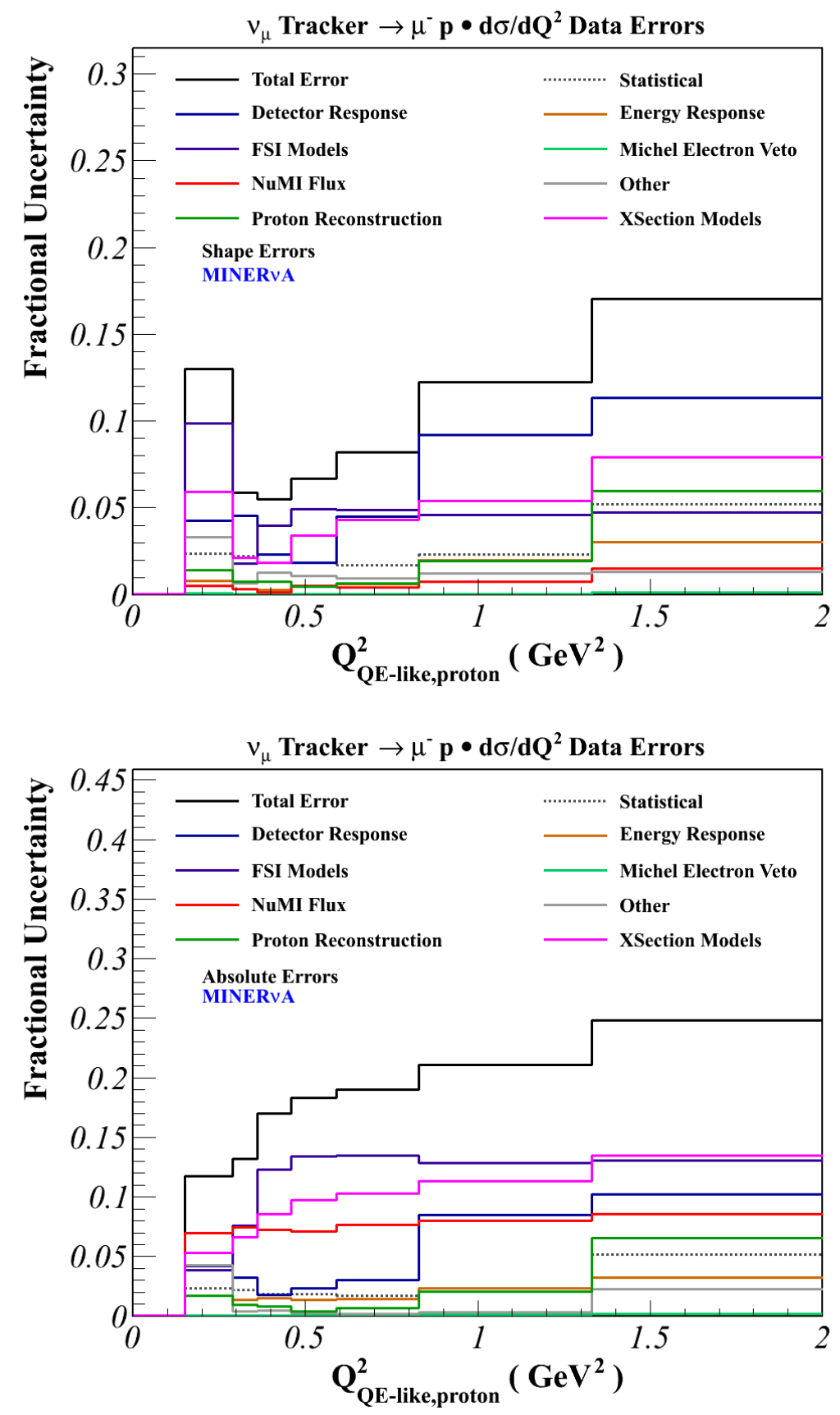

Figure 5.34. Plotted are the shape (top) and absolute (bottom) error summary for the $\mathrm{d} \sigma / \mathrm{dQ}_{\mathrm{QE}-\text { like,proton }}^{2}$ with $\mathrm{p}_{\text {proton }}>450 \mathrm{MeV} / \mathrm{c}$. 
Table 5.6. Fractional systematic uncertainties on the $\mathrm{d} \sigma / \mathrm{dQ}_{\mathrm{QE}-\text {-like,proton }}^{2}$ measurement with $\mathrm{p}_{\text {proton }}>450 \mathrm{MeV} / \mathrm{c}$ for each $\mathrm{Q}_{\mathrm{QE} \text {,proton }}^{2}$ bin. Both the absolute (top) and shape (bottom) errors are presented for each $\mathrm{Q}_{\mathrm{QE} \text {,proton }}^{2}$ bin and the contributors are (I) flux, (II) neutrino interaction models, (III) final state interaction models, (IV) detector response (V) energy response, (VI) proton reconstruction, (VII) Michel electron veto, and (VIII) other

\begin{tabular}{|c||cccccccc|}
\hline \hline \multicolumn{10}{|c|}{ Absolute Errors } \\
\hline $\mathrm{Q}_{\mathrm{QE} \text {,proton }}^{2}\left(\mathrm{GeV}^{2}\right)$ & I & II & III & IV & V & VI & VII & VIII \\
\hline $0.15-0.29$ & 0.069 & 0.053 & 0.038 & 0.042 & 0.017 & 0.017 & 0. & 0. \\
$0.29-0.36$ & 0.075 & 0.066 & 0.076 & 0.033 & 0.014 & 0.004 & 0.001 & 0. \\
$0.36-0.46$ & 0.073 & 0.086 & 0.123 & 0.018 & 0.015 & 0.008 & 0.001 & 0. \\
$0.46-0.59$ & 0.071 & 0.097 & 0.134 & 0.023 & 0.015 & 0.004 & 0.001 & 0. \\
$0.59-0.83$ & 0.076 & 0.103 & 0.134 & 0.030 & 0.014 & 0.007 & 0.001 & 0. \\
$0.83-1.33$ & 0.080 & 0.114 & 0.128 & 0.085 & 0.015 & 0.021 & 0.001 & 0. \\
$1.33-2.0$ & 0.086 & 0.135 & 0.130 & 0.102 & 0.023 & 0.065 & 0.002 & 0. \\
\hline \multicolumn{10}{|c}{ Shape Errors } \\
\hline $0.15-0.29$ & 0.005 & 0.054 & 0.099 & 0.042 & 0.008 & 0.014 & 0. & 0.001 \\
$0.29-0.36$ & 0.003 & 0.021 & 0.018 & 0.046 & 0.003 & 0.008 & 0. & 0. \\
$0.36-0.46$ & 0.001 & 0.018 & 0.040 & 0.023 & 0.003 & 0.007 & 0. & 0. \\
$0.46-0.59$ & 0.005 & 0.034 & 0.049 & 0.018 & 0.005 & 0.005 & 0. & 0. \\
$0.59-0.83$ & 0.004 & 0.043 & 0.046 & 0.045 & 0.006 & 0.006 & 0. & 0. \\
$0.83-1.33$ & 0.008 & 0.054 & 0.047 & 0.092 & 0.020 & 0.019 & 0. & 0.001 \\
$1.33-2.0$ & 0.015 & 0.079 & 0.080 & 0.113 & 0.030 & 0.060 & 0.001 & 0.001 \\
\hline
\end{tabular}

Table 5.7. Flux-integrated $\mathrm{d} \sigma / \mathrm{dQ}_{\mathrm{QE}-\text { like,proton }}^{2}$ Summary for $\mathrm{p}_{\text {proton }}>450 \mathrm{MeV} / \mathrm{c}$. Both the statistical (first) and full systematic (second) errors are presented for each measurement.

\begin{tabular}{c||cc}
\hline \hline $\begin{array}{c}\mathrm{Q}_{\mathrm{QE}, \text { proton }}^{2} \\
\left(\mathrm{GeV}^{2}\right)\end{array}$ & $\begin{array}{c}\text { Cross-section }\left(\mathrm{p}_{\text {proton }}>450 \mathrm{MeV} / \mathrm{c}\right) \\
\left(10^{-38} \mathrm{~cm}^{2} / \mathrm{GeV}^{2} / \text { nucleon }\right)\end{array}$ & $\begin{array}{c}\text { Cross-section } \\
\left(10^{-38} \mathrm{~cm}^{2} / \mathrm{GeV}^{2} / \text { nucleon }\right)\end{array}$ \\
\hline $0.0-0.15$ & 0. & $1.106 \pm 0.074 \pm 0.241$ \\
$0.15-0.29$ & $0.594 \pm 0.014 \pm 0.069$ & $0.843 \pm 0.019 \pm 0.088$ \\
$0.29-0.36$ & $0.700 \pm 0.015 \pm 0.091$ & $0.699 \pm 0.015 \pm 0.091$ \\
$0.36-0.46$ & $0.563 \pm 0.010 \pm 0.095$ & $0.561 \pm 0.010 \pm 0.095$ \\
$0.46-0.59$ & $0.421 \pm 0.008 \pm 0.077$ & $0.420 \pm 0.008 \pm 0.077$ \\
$0.59-0.83$ & $0.293 \pm 0.005 \pm 0.055$ & $0.293 \pm 0.005 \pm 0.055$ \\
$0.83-1.33$ & $0.138 \pm 0.003 \pm 0.029$ & $0.138 \pm 0.003 \pm 0.028$ \\
$1.33-2.0$ & $0.060 \pm 0.003 \pm 0.015$ & $0.060 \pm 0.003 \pm 0.015$ \\
\hline
\end{tabular}


in the cross section measurement for $0.15<\mathrm{Q}_{\mathrm{QE} \text {,proton }}^{2}<0.29$. This is primarily due to the efficiency-correction procedure. The measurements are identical for $\mathrm{Q}_{\mathrm{QE} \text {,proton }}^{2}$ values greater than $0.29 \mathrm{GeV}^{2}$. Thus, it can be assumed that the cross section $\mathrm{d} \sigma / \mathrm{dQ}_{\mathrm{QE}-\text { like,proton }}^{2}$ that is produced for $\mathrm{p}_{\text {proton }}>450 \mathrm{MeV} / \mathrm{c}$ is insensitive to the reconstruction inefficiencies which are explained in Subsection 5.3.2. The cross section measurement produce with the condition on the proton's momentum is interpreted in Chapter 6 and the systematic uncertainties on the data $\mathrm{d} \sigma / \mathrm{dQ}_{\mathrm{QE}-\text { like,proton }}^{2}$ is discussed next.

\subsection{SYSTEMATIC UNCERTAINTIES}

The various components which are needed to produce the $\mathrm{d} \sigma / \mathrm{d} \mathrm{Q}_{\mathrm{QE}-\text { like,proton }}^{2}$ measurement have been presented throughout this dissertation. Simulations are utilized to model these components which includes the incoming neutrino beam, the neutrino interactions in nuclear matter, and the particle propagation through the detector. Each analysis step which is outlined in the previous section depends on these simulated quantities, which are known to be imperfect. The estimated uncertainties on the various modeling contributions, give rise to the systematic errors.

As mentioned earlier, the systematic errors are evaluated by applying a procedure known as the many universes [92]. For a given systematic source, the relevant model's parameters are shifted within their measured $\pm 1 \sigma$ uncertainties. The measured observable is then re-extracted with the complete analysis procedure using the shifted parameters. This variation is commonly referred to as an universe, which represents the deviation from the measured nominal value. In most cases, the parameters are varied by $\pm 1 \sigma$. There are cases where the error on the systematic source has been reproduced using 100 variations, where the shifted values are randomly drawn from sampling a Gaussian distribution with a width of a $1 \sigma$. The purpose for using 100 variations is to minimize the statistical uncertainties on the systematic uncertainties. 
For all cases, the systematic errors are computed using a covariance matrix, where the calculation depends on the number of universes.

- For the case with one variation, the covariance matrix is given by,

$$
C_{i j}=\left(x_{\text {universe }, i}-x_{i}\right)\left(x_{\text {universe }, j}-x_{j}\right) .
$$

where $\mathrm{x}_{i}$ is the $i^{\text {th }}$ bin of the nominal value and $\mathrm{x}_{\text {universe }, i}$ is the $i^{\text {th }}$ bin of the value with the varied parameter.

- For all other cases, the covariance matrix is calculated as,

$$
C_{i j}=\frac{1}{N} \sum_{k=1}^{N}\left(x_{k, i}-\bar{x}_{i}\right)\left(x_{k, j}-\bar{x}_{j}\right),
$$

where $\mathrm{N}$ is the total number of variations, $\mathrm{x}_{k, i}$ is the $i^{\text {th }}$ bin of the $k^{\text {th }}$ variation and $\bar{x}_{i}$ is the $i^{t h}$ bin of the average of the measured value for all variations.

As presented throughout this chapter, the systematic errors are produced as two ways: the shape and absolute errors. Both the shape and absolute errors are computed as describe above. In the case of the shape errors, the covariance matrix is area normalized before it is built. This means that each Monte Carlo universe distribution is scaled by a normalization factor that makes the integrated Monte Carlo universe equals the integrated nominal Monte Carlo. For both the shape and absolute errors, the systematic uncertainty for a given source is the square-root of the diagonal elements of the systematic source total covariance matrix.

\subsubsection{NEUTRINO FLUX}

One of the biggest challenges for all neutrino experiments is to understand the predicted neutrino flux. Recall that $\operatorname{MINER} \nu \mathrm{A}$ is exposed to a tertiary neutrino beam, which is the final product of the $p C$ collisions. Therefore, the simulation of the predicted neutrino flux depends heavily on the modeling of the $p C$ interactions and the focusing of these produced hadrons along the beamline, as well as these 
hadrons re-interactions in the target-horn system. The primary contributions to the uncertainty on the predicted neutrino flux are listed below.

- The primary constrained hadron production data. The simulated $p C$ interaction is constrained using external hadron production data that is described in Section 4.2.1, where the NA49 data is the primary dataset used to constrain the produced pions. Since NA49 experiment uses a $158 \mathrm{GeV}$ proton beam, it's data is related to NuMI's proton beam $(120 \mathrm{GeV})$ through Feynman scaling [93]. The systematic uncertainty on the constrained hadron production data comes from uncertainty in the scaling procedure [94].

- The hadron production model. Recall that the external hadron production data is applied to a particular $p C$ reaction the occurs within defined kinematic space which is a function of Feynman $\mathrm{x}_{F}$ and $\mathrm{p}_{T}$. Thus, systematic uncertainty on the $p C$ interactions that are not constrained, are evaluated by comparing the various hadronic physics models (see Figure 4.11) description of the interactions.

- The NuMI beamline. The systematic uncertainties on the NuMI beamline were evaluated and provided by the MINOS experiment [95]. The sources which contribute to the total uncertainty are summarized in Table 5.4.1.

Table 5.8. The systematic uncertainties on various sources of the NuMI beamline. Reprinted from Reference [95], Copyright (2008).

\begin{tabular}{c||c}
\hline \hline Sources & Uncertainty \\
\hline \hline Number of protons on target & $2.0 \%$ \\
Horn transverse misalignment & $1.0 \mathrm{~mm}$ \\
Horn tilt & $0.2 \mathrm{mrad}$ \\
Horn current mis-calibration & $1.0 \%$ \\
Horn current distribution & $\delta=6 \mathrm{~mm} / \delta=\infty$ \\
Baffle scraping & $0.25 \%$ \\
Mis-alignment of shielding blocks & $1.0 \mathrm{~cm}$ \\
Target density & $2.0 \%$ \\
\hline
\end{tabular}

The contributions to the total uncertainty on the predicted neutrino flux are assumed to be uncorrelated. Figure 5.35 summarizes these various sources for the analysis presented. 


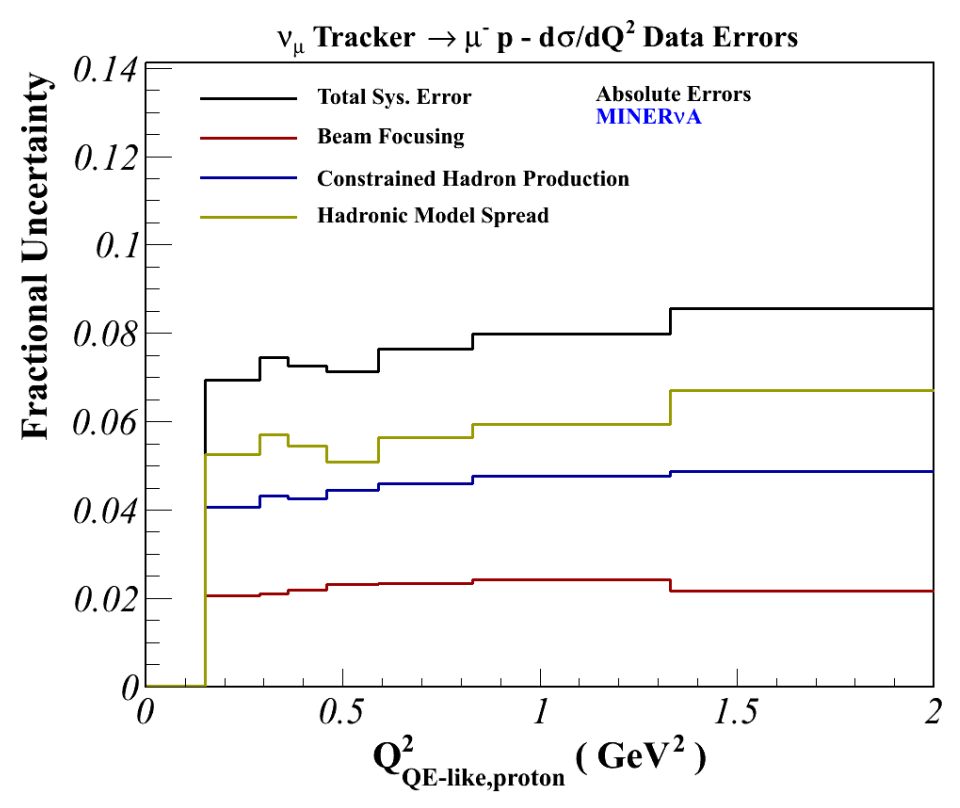

Figure 5.35. The predicted neutrino flux error summary. Recall that unlike other MINER $\nu \mathrm{A}$ analyses, this analysis does not cut on the reconstructed neutrino energy.

\subsubsection{GENIE}

GENIE is utilized to simulate the neutrino interactions observed in the MINER $\nu \mathrm{A}$ detector. The differential cross section measurement is sensitive to GENIE models, because of it's reliance on GENIE for the background subtraction, the detector unfolding, and efficiency correction. Recall that a data-driven procedure is implemented for tuning the non QE-like backgrounds, where the procedure assumes that GENIE correctly predicts the rate for each neutrino interaction process for each of the sidebands. For the unfolding procedure, the unfolding matrix is composed of the $\nu_{\mu}$ QE-like events, which depends on GENIE modeling of the FSI effects. The efficiency function is also dependent on GENIE modeling of the $\nu_{\mu}$ QE-like events. Therefore, GENIE modeling of the $\nu_{\mu}$ QE-like signal and backgrounds, as well as the hadron transportation through the nucleus all contribute to the systematic uncertainty on the measured cross section. Table 5.4.2 provides a list of the most significant contributors to the 
GENIE systematic, along with it's $\pm 1 \sigma$ uncertainty. The data $\mathrm{d} \sigma / \mathrm{dQ}_{\mathrm{QE}-\text { like,proton }}^{2}$ error summary of the parameters which contributes the most to the GENIE modeling systematic uncertainty are displayed in Figure 5.36. The systematic errors on the parameters which control the modeling of the QE cross section (the middle plot) are negligible. The resonant cross section free parameter, $\mathrm{M}_{A}$ with an systematic uncertainty of $\sim 7-8 \%$ does contributes to the GENIE systematic uncertainty. The biggest contributors to the GENIE systematic are from the INC modeling of the pion inelastic and absorption cross sections, as indicated in the top plot in Figure 5.36.

These parameters' uncertainties (shown in Table 5.4.2) on GENIE prediction of the neutrino processes and hadrons propagation through the atomic nucleus are evaluated by simulateously shifting each model's parameters by $\pm 1 \sigma$. The events are re-weighted to account for the tweaking of the parameters that control the GENIE models. Note that both the MINOS and T2K experiments have extensively studied the parameters which account for uncertainties for the GENIE cross section models [96].

\subsubsection{GEANT4}

Geant4 simulates particles propagating through the MINER $\nu$ A detector. Therefore, the Geant4 modeling of the particle's energy response and the particle's interactions with the detector's material produce an uncertainty on the energy deposition for the predicted unattached visible energy and the hadron tracks that are reconstructed in the detector. The estimated uncertainty on the energy deposition of the particles is utilized to obtain a systematic uncertainty on the unattached visible energy. Monte Carlo samples with the Geant4 modeling of the total inelastic cross section shifted for both the protons and pions, are evaluated to retrieve an uncertainty on Geant4 contribution to the proton reconstruction systematic. This section sumarizes the uncertainties that contribute to the detector response systematic due to the Geant4 models. 
Table 5.9. The systematic uncertainties on various parameters for GENIE models. Reprinted from Reference [75], Copyright (2010).

\begin{tabular}{|c|c|c|}
\hline "GENIE Model Parameters & GENIE Knob name & $1 \sigma$ \\
\hline \multicolumn{3}{|c|}{ Cross Section Models } \\
\hline $\mathrm{M}_{A}$ for CC QE Scattering & $\mathrm{MaCCQE}$ & $\pm 10 \%$ \\
\hline $\mathrm{M}_{A}$ for Resonance & MaRES & $\pm 20 \%$ \\
\hline $\mathrm{M}_{V}$ for Resonance & MvRES & $\pm 10 \%$ \\
\hline CC QE Normalization & NormCCQE & $+20 \%-15 \%$ \\
\hline CC Resonance Normalization & NormCCRES & $\pm 20 \%$ \\
\hline $1 \pi$ Production from $\nu_{\mu}$ p non-resonant & Rvp1pi & $\pm 50 \%$ \\
\hline $1 \pi$ Production from $\bar{\nu}_{\mu} \mathrm{n}$ non-resonant & Rvn1pi & $\pm 50 \%$ \\
\hline $2 \pi$ Production from $\nu_{\mu}$ p non-resonant & Rvp2pi & $\pm 50 \%$ \\
\hline $2 \pi$ Production from $\bar{\nu}_{\mu} \mathrm{n}$ non-resonant & Rvn2pi & $\pm 50 \%$ \\
\hline \multicolumn{3}{|c|}{ IntraNuclear Cascade Models } \\
\hline Nucleon Elastic Scattering & FrElasN & $\pm 30 \%$ \\
\hline$\pi$ Elastic Scattering & FrElaspi & $\pm 10 \%$ \\
\hline Nucleon Inelastic Scattering & FrInelN & $\pm 40 \%$ \\
\hline$\pi$ Inelastic Scattering & FrInelpi & $\pm 40 \%$ \\
\hline Nucleon Absorption & FrAbsN & $\pm 20 \%$ \\
\hline$\pi$ Absorption & FrAbspi & $\pm 30 \%$ \\
\hline Nucleon Mean Free Path & MFPN & $\pm 20 \%$ \\
\hline$\pi$ Mean Free Path & MFPpi & $\pm 20 \%$ \\
\hline
\end{tabular}



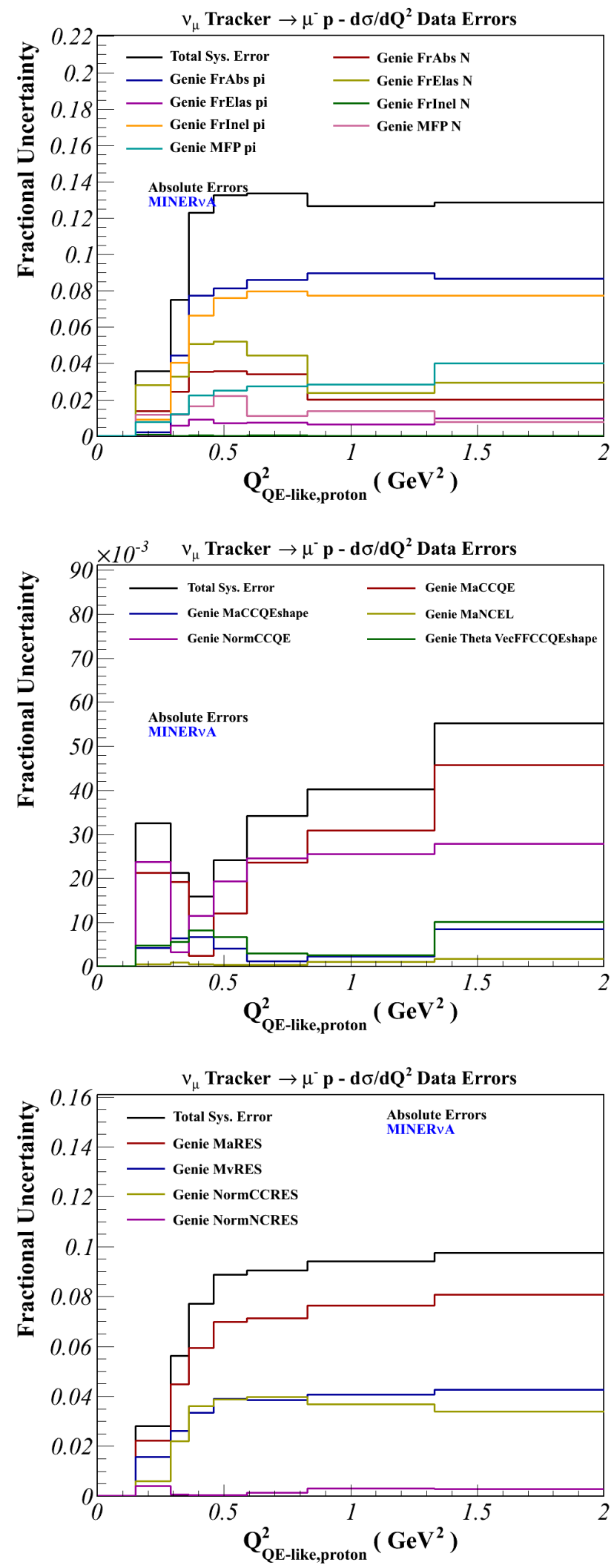

Figure 5.36. Plotted are the most significant error sources for this analysis from GENIE cross section and final state interaction models. 


\section{The Uncertainty on the Energy Response}

Recall that the $\nu_{\mu}$ QE-like candidates are isolated using the unattached visible energy observable, and this cut is sensitive to the observed scintillation light that is produced by the particle. The simulated particles which produce the energy that is apart of the unattached visible energy system, are utilized to evaluate the uncertainty on the energy response. The Monte Carlo identifies each particle that produces the hits that is apart of the unattached visible energy system. Then, the simulated hits are scaled by the particle's response uncertainties, and which is given in Table 5.10. For information on the extraction of the uncertainty produced by each particle source, see References [53] and [55]. The energy shift for a given particle is the difference between the nominal and scaled unattached visible energy. The systematic uncertainty (see Figure 5.37) on each particle's energy response is obtained by sampling the energy shift in a Gaussian distribution with a width of $1 \sigma$. All of these sources contribute insignificantly to the total systematic uncertainty on the cross section.

\begin{tabular}{c||c} 
Table 5.10. The $\mathrm{E}_{\text {unattach }}^{\text {vis }}$ Energy Response Uncertainti \\
\hline \hline Particle Sources & Uncertainty \\
\hline \hline proton & $3.5 \%$ \\
neutron $\left(\mathrm{E}_{\text {kin }}<50 \mathrm{MeV}\right)$ & $25 \%$ \\
neutron $\left(50<\mathrm{E}_{\text {kin }}<150 \mathrm{MeV}\right.$ & $10 \%$ \\
neutron $\left(\mathrm{E}_{k i n}>150 \mathrm{MeV}\right.$ & $20 \%$ \\
muon & $2.4 \%$ \\
$\gamma, \pi^{0}, e^{ \pm}$ & $3 \%$ \\
$\pi^{ \pm}$, Kaon & $5 \%$ \\
optical cross talk & $20 \%$ \\
other & $20 \%$ \\
\hline
\end{tabular}

Hadrons transversing the detector can undergo nuclear re-interactions or become absorbed within the nuclear matter. For these cases, $d E / d x$ particle identification algorithm is more likely to perform inefficiently for identifying the particle's type. This is because the direction of the particle's trajectory can abruptly change, the particle can produce a cascade of secondary particles, or be absorbed by the detector's 


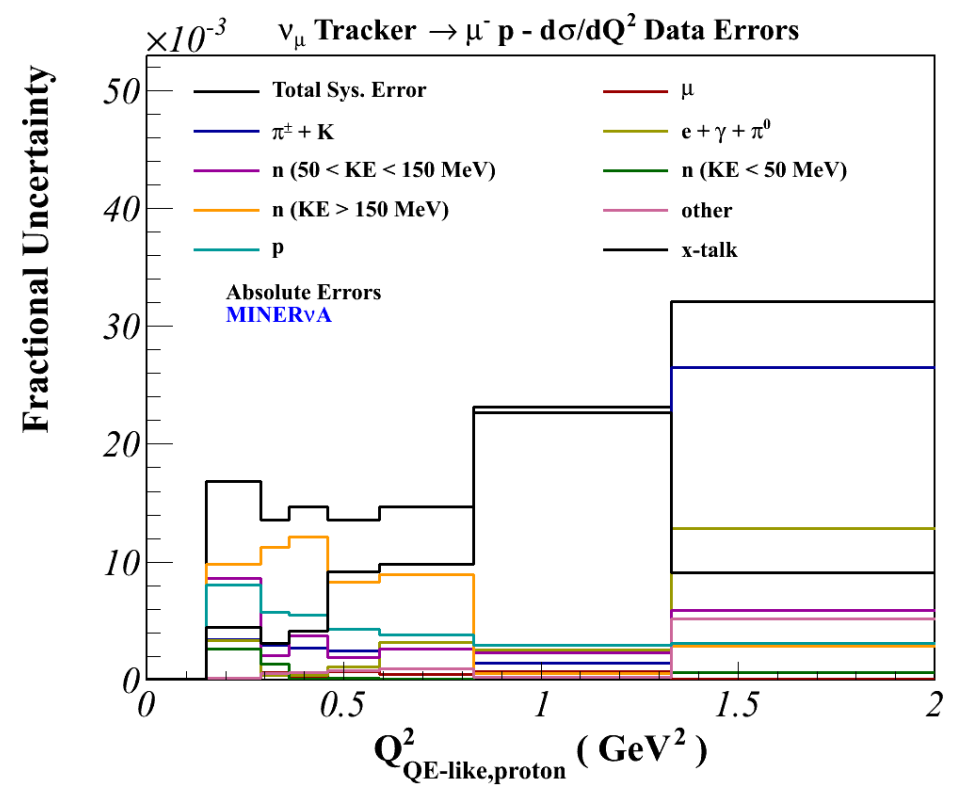

Figure 5.37. The cross section absolutely errors for each particle's source which contributes to the systematic uncertainty on the detector response for the unattached visible energy.

materials. The reconstruction and the identification of hadrons are sensitive to Geant4 modeling of these reactions in the detector. As mentioned above, alternative Monte Carlo samples with the hadron total inelastic cross section biased by $\pm 10 \%$. These samples are utilized to evaluate an uncertainty on Geant4 modeling of the hadron transportation in the detector. The $\pm 10 \%$ errors are determined from the analysis which compares the Geant4 cross section predictions and to hadron production data on Carbon and Iron [97]. The systematic uncertainties on the $\mathrm{d} \sigma / \mathrm{d} \mathrm{Q}_{\mathrm{QE}-\text { like,proton }}^{2}$ due to the Geant4 modeling of the hadron inelastic cross sections are presented in Figure 5.38. The uncertainty on the pion inelastic cross section becomes significant at high $\mathrm{Q}_{\mathrm{QE} \text {,proton }}^{2}$. The proton inelastic cross section has an overall uncertainty that is less than $4 \%$, where the $\mathrm{Q}_{\mathrm{QE} \text {,proton }}^{2} \in[0.8-1.4] \mathrm{GeV}^{2}$ has an systematic uncertainty of $\sim 8 \%$. 


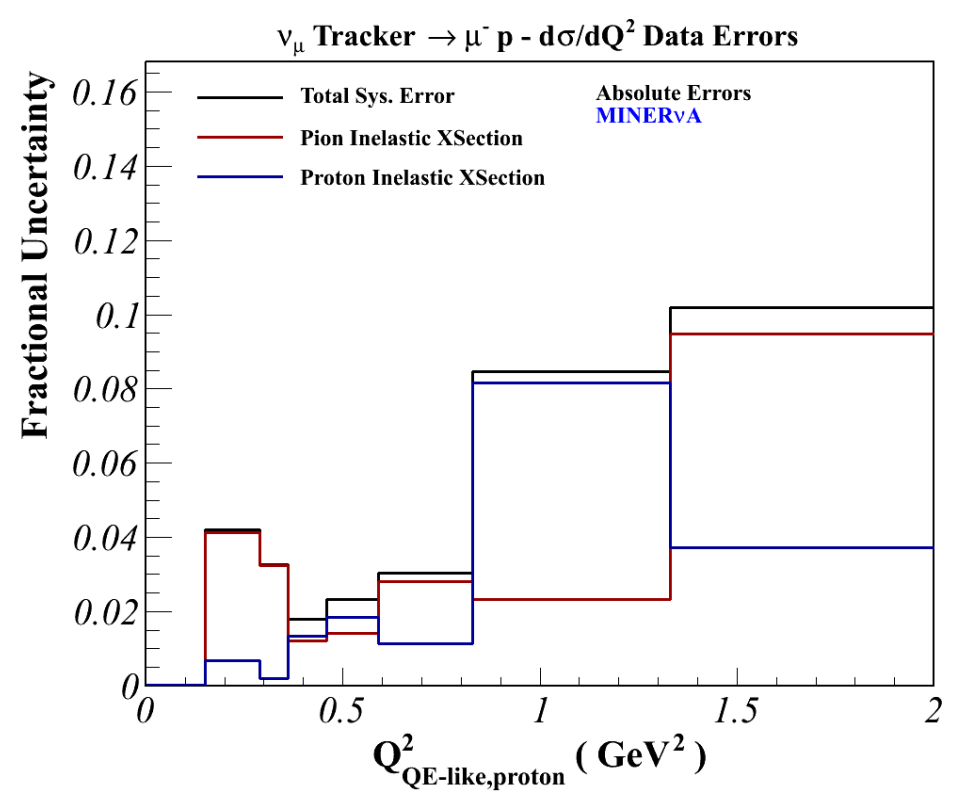

Figure 5.38. The systematic uncertainties on the cross section that are due to the Geant4 modeling of the proton and pion inelastic cross sections.

\subsubsection{PROTON RECONSTRUCTION}

Recall that $d E / d x$ profile along the track and the track's range are used simultaneously to reconstruct the momentum and determine the particle's type of the hadron track candidate. The additional sources of uncertainties on the detector's response which contribute to the uncertainty on the proton reconstruction systematic, include the following:

- Bethe-Bloch calculation. The particle creation algorithms uses the BetheBloch formula to calculate the average energy loss in a material. Based on studies which compares the muon energy loss in the detector to the Groom's muon energy range tables, the uncertainity on the Bethe-Bloch calcuation is approximately 1\%(3\%) for a given pure(compound) material [98]. Thus, the calculated $d E / d x$ for a given track is scaled by $1 \%(3 \%)$ when the track tranverses the MINER $\nu$ A detector's materials(Titianium Dioxide mixture), respectively.

- Detector mass model. The energy reconstruction depends on the path $(d x)$ of the material that the track traverses. The $d x$ of the material which the track traverses, is scaled up and down by the systematic uncertainty on the thickness of the materials in the MINER $\nu \mathrm{A}$ detector, and the uncertainty on material's budget is summarized in Table 5.11. 
- Absolute energy scale constant (MEU). The measured $d E / d x$ is sensitive to absolute energy scale calibration, which has a systematic uncertainty of $2.4 \%$ [99]. Therefore, the cluster's energy on a track is shifted by $\pm 2.4 \%$.

- Birk's Law Quenching parameter $\left(\mathbf{k}_{B}\right)$. The photoelectron's light yield is sensitive to the Birk's quenching affects. The data analysis of stopping protons observed from the Fermilab Mesons Test Beam Facility (T977), measured the Birk's parameter for the scintillator planes to be $\mathrm{k}_{B}=0.0905 \pm 0.012 \mathrm{~m} / \mathrm{GeV}$, where the Monte Carlo takes the value as $\mathrm{k}_{B}=0.133 \mathrm{~m} / \mathrm{GeV}$ [100]. The difference between the measured and Monte Carlo values is a systematic uncertainty on the Birk's parameter. To evaluate the effect of the uncertainty on the Birk's parameter for the proton reconstruction, each Monte Carlo hit on a given track is modified by $\Delta \mathrm{E}_{\text {hit }}$, which accounts for the shift between the measured and Monte Carlo $\mathrm{k}_{B}$ parameter [101]. Recall that the Birk's Law can be written as,

$$
d E=\frac{d E^{\prime}}{1+k_{B} d E^{\prime} / d x}
$$

and the $\mathrm{O}(1)$ correction is approximated using a Taylor's expansion series, which is given by,

$$
\Delta E_{\text {hit }}=\frac{-E_{\text {hit }}^{2}}{\mathrm{x}_{\text {hit }}} \delta,
$$

where $\mathrm{E}_{\text {hit }}$ and $\mathrm{x}_{\text {hit }}$ are the energy deposited and path length of the Monte Carlo hit for a given particle and the difference between the measured and Monte Carlo $\mathrm{k}_{B}$ is $\delta=-0.0545 \mathrm{~m} / \mathrm{GeV}[101]$.

The systematic uncertainties on the $\mathrm{d} \sigma / \mathrm{dQ}_{\mathrm{QE}-\text { like,proton }}^{2}$ for each of these given sources are summarized in Figure 5.39. The detector response for the proton reconstruction does not contribute significantly to the total systematic uncertainty on the cross section. This is due to the robustness of the $d E / d x$ reconstruction algorithm. Recall that this analysis incorporates a simple outlier removal (see Section 4.3.5) technique which discard clusters with large energy deposition from the fitting procedure in order to optimize the calculation of the particle's score. This procedure seems to reduce the systematic uncertainties which comes from the detector's response. In addition, the systematic uncertainty is negligible in the low $\mathrm{Q}_{\mathrm{QE} \text {,proton }}^{2}$ regime, which is due to choosing appropriate bin edges to prevent bin migration. 
Table 5.11. The Mass Thickness Uncertainties for Material in the Tracker Region. Reprinted from Reference [102].

\begin{tabular}{c|c|c|c}
\hline Plastic Scintillator & Carbon & Iron & Lead \\
\hline \hline $1.4 \%$ & - & $0.5 \%$ & $1.3 \%$ \\
\hline
\end{tabular}

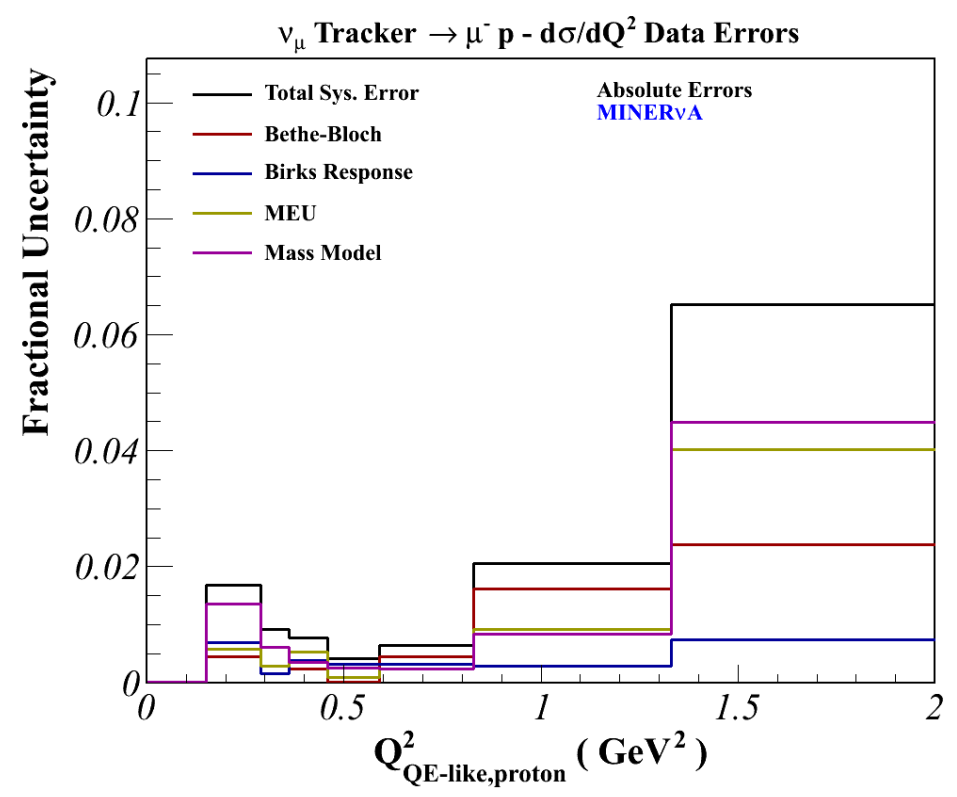

Figure 5.39. Fractional uncertainty on the cross section due to various source that contribute to proton reconstruction systematic uncertainty. 


\subsubsection{MICHEL ELECTRON VETO}

The final analysis cut in the $\nu_{\mu}$ QE-like selection serves to veto events with a Michel electron, where the Michel electron is produced by the decays of charged pions in the final state. The reconstruction of the Michel electron affects the efficiency and the background subtraction. The efficiency of the Michel electron reconstruction is evaluated using stopping rock muons, and the efficiency uncertainty is determined to be $\sigma_{\text {effmichel }}=1 \%$ [103]. For the analysis presented, this $\sigma_{\text {effmichel }}$ is utilized to re-weight all simulated events which have a Michel electron, by a weighting factor

$$
\mathrm{w}_{\text {effmichel }}=\left(1.0 \pm \frac{\sigma_{\text {effmichel }}}{1.0}\right)
$$

Note that the origin of the Michel electron and the topology of the charged pion are neglected. In order to obtain a more accurate systematic uncertainty on the Michel electron veto performance for both the data and Monte Carlo, the systematic error needs to be evaluated utilizing pions. Since events with pions have more activity, which can produce detector after-pulsing, it is expected that the uncertainty on the Michel electron veto may be greater than $1 \%$. In addition, the Geant4 modeling of the charged pion transportation in the detector, also contributes to the uncertainty on the efficiency for finding Michel electrons.

As mentioned above, the background subtraction may also be sensitive to the Michel electron reconstruction efficiency. The Michel electron veto changes the overall background event rate. To determine an uncertainty on background subtraction sensitivity to the Michel electron veto, the two-component background scale factors (see Subsection 5.3.1) are extracted with and without applying the Michel electron veto. The differences between the extracted scale factors are taken to be the uncertainty due to Michel electron veto on the background subtraction. These uncertainties on the background scale factors are $1.6 \%(3.5 \%)$ for the resonant(DIS plus other) component. The systematic error on the extracted cross section is evaluated by re-weighting 
the two-component backgrounds that produces a true Michel electron. The systematic uncertainties on the $\mathrm{d} \sigma / \mathrm{dQ}_{\mathrm{QE}-\text { like,proton }}^{2}$ for the Michel electron given sources are summarized in Figure 5.40.

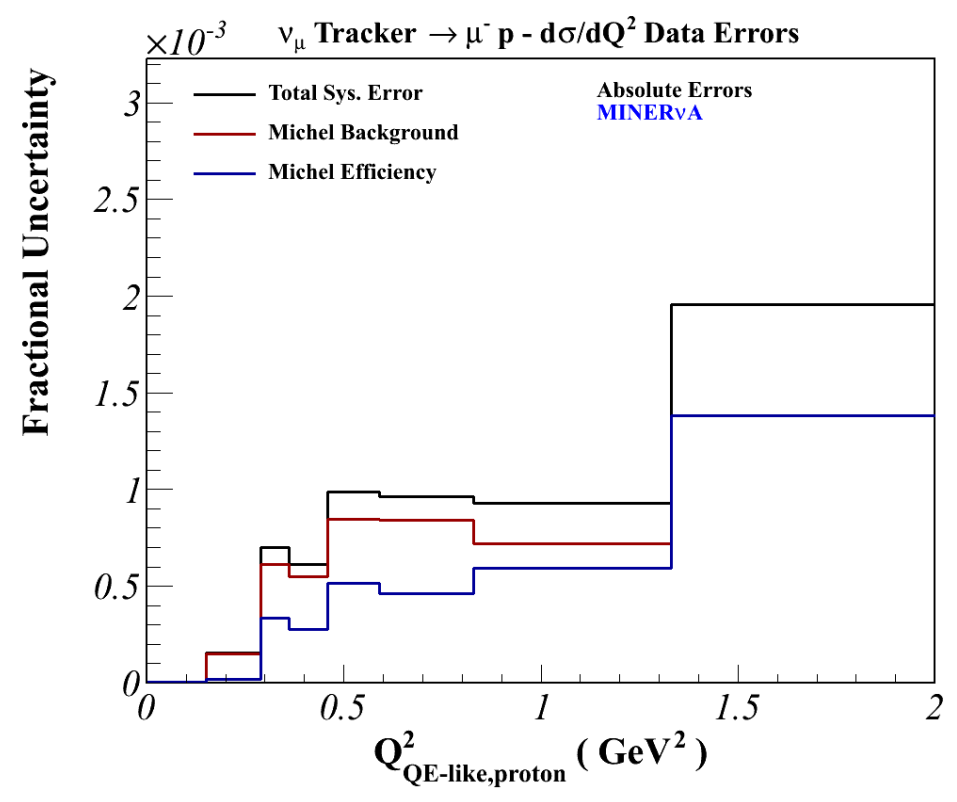

Figure 5.40. The Michel electron veto systematic uncertainty. The systematic error on the Michel electron reconstruction is negligible.

\subsubsection{OTHER SYSTEMATIC UNCERTAINTIES}

Other sources which contribute to the total systematic uncertainty include the following:

- A flat correction is applied to account for the detector mass scale with $\delta= \pm 1.4 \%$.

- The systematic uncertainty on the unfolding technique is evaluated by unsmearing the reconstructed events using five iterations of the Bayesian unfolding iterative procedure. The systematic uncertainty is the maximum deviation between the nominal $\mathrm{d} \sigma / \mathrm{dQ}_{\mathrm{QE}-\text { like,proton }}^{2}$ and the $\mathrm{d} \sigma / \mathrm{dQ}_{\mathrm{QE}-\text { like,proton that is pro- }}^{2}$ duced using the Bayesian unfolding procedure with five iterations.

These other contributions to the total systematic uncertainty on the $\mathrm{d} \sigma / \mathrm{d} \mathrm{Q}_{\mathrm{QE}-\text { like,proton }}^{2}$ are summarized in Figure 5.41. 


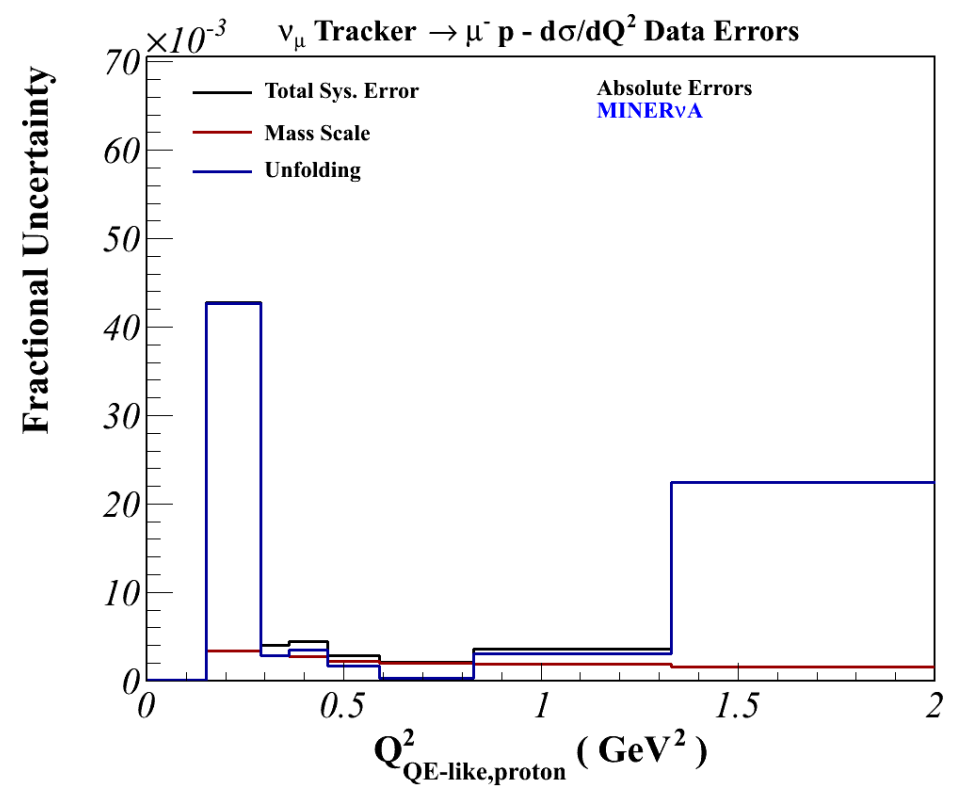

Figure 5.41. The remaining contributions to the total systematic uncertainty.

The remaining systematic sources which need to be studied for this analysis, include the following:

- The proton track efficiency.

- The pID performance.

- The reconstruction of the proton's angle.

These contributors to the systematic uncertainty are in the process of being evaluated. Recall that the proton track efficiency uncertainty will be determined from the scanning of both data and Monte Carlo events. The uncertainty on the efficiency for the pID and the proton's angle reconstruction will be determined from the analysis of protons observed by the Fermilab Meson Test Beam Facility. 


\subsection{STUDYING NUCLEAR EFFECTS USING BOTH THE MUON AND PROTON KINEMATICS}

The $\nu_{\mu}$ QE-like cross section for this analysis is measured using only the proton kinematics, as indicated in Equation 5.2.1. Recall that $\mathrm{Q}_{\mathrm{QE} \text {,proton }}^{2}$ is derived assuming $\nu_{\mu}$ QE scattering from a free nucleon at rest. This calculation does not take into account any of the scattering nuclear physics which has been mentioned repeatedly throughout this dissertation. The measured $\mathrm{d} \sigma / \mathrm{dQ}_{\mathrm{QE}-\text { like,proton }}^{2}$ is sensitive to the GENIE modeling of the following nuclear effects.

- The Fermi motion which describes the momentum of nucleons bound in a nuclear potential.

- The effects of FSIs which accounts for the missing energy of the final state nucleons.

It is essential to understand how these nuclear effects distort the shape of the measured $\mathrm{d} \sigma / \mathrm{dQ}_{\mathrm{QE}-\text { like,proton }}^{2}$ distribution.

A subset of the $\nu_{\mu}$ QE-like candidates consists of events where both the muon and leading proton have a sensible reconstructed energy, where leading refers to the most energetic proton. Recall that observables that are reconstructed from the muon are insensitive to the Fermi motion and effects of FSIs. Using the QE hypothesis, various kinematic observables, which require both the muon and proton information, can be reconstructed. These observables evaluate the simulation modeling of the nuclear effects that are described above. This section summarizes the analysis that is used to interpret the $\mathrm{d} \sigma / \mathrm{dQ}_{\mathrm{QE}-\text { like,proton }}^{2}$ sensitivity to the effects of FSI by analyzing the event kinematics that are reconstructed using both the final state lepton and hadron observables. 


\subsubsection{GENIE INTERPRETATION OF NUCLEAR EFFECTS}

The reconstructed observables are smeared due to the models in the neutrino event simulation and finite detector resolution. In this section, the event kinematics are analyzed using only GENIE truth information. Thus, the observables are insensitive to the reconstruction inefficiencies. The distortions due to nuclear effects are analyzed by studying the correlations between the $\mathrm{Q}^{2}$ computed from the muon and leading proton or all of the particles in the hadronic final state. The various way to calculate the $\mathrm{Q}^{2}$ are listed below.

\section{- lepton kinematic}

$$
Q^{2}=-q^{2}=\left(p^{\nu}-p^{\mu}\right)^{2},
$$

where $\mathrm{p}^{\nu}$ and $\mathrm{p}^{\mu}$ are the four-momentum vector of the incoming neutrino and scattered muon.

- nucleon kinematic

$$
Q^{2}=-q^{2}=\left(p^{n}-p^{p}\right)^{2},
$$

where $\mathrm{p}^{n}$ and $\mathrm{p}^{p}$ are the four-momentum vector of the struck neutron and scattered proton.

- event kinematic

$$
Q^{2}=-m_{\mu}^{2}+2 E_{\nu}\left(E_{\mu}-\sqrt{E_{\mu}^{2}-m_{\mu}^{2}} \cos \theta_{\mu}\right),
$$

where $\mathrm{m}_{\mu}, \theta_{\mu}$, and $\mathrm{E}_{\mu}$ are the mass, scattering angle, and energy of the muon, and $\mathrm{E}_{\nu}$ is the energy of the neutrino.

- muon kinematic

$$
Q^{2}=-m_{\mu}^{2}+2 E_{\nu}^{Q E}\left(E_{\mu}-\sqrt{E_{\mu}^{2}-m_{\mu}^{2}} \cos \theta_{\mu}\right),
$$

where the difference between the muon and event kinematics is the calculation of the neutrino energy, $\mathrm{E}_{\nu}$,

$$
E_{\nu}^{Q E}=\frac{2\left(M_{n}-E_{B}\right) E_{\mu}-\left[\left(M_{n}-E_{B}\right)^{2}+m_{\mu}^{2}-M_{p}^{2}\right]}{2\left[M_{n}-E_{B}-E_{\mu}-p_{\mu} \cos \theta_{\mu}\right]},
$$

where $\mathrm{M}_{n}$ and $\mathrm{M}_{p}$ are the masses of the neutron and proton and the binding energy, $\mathrm{E}_{B}$ is $34.0 \mathrm{MeV}$. 
- proton kinematic

$$
Q^{2}=\left(M^{\prime}\right)^{2}-M_{p}^{2}+2 M^{\prime}\left(E_{k i n}+M_{p}-M^{\prime}\right)
$$

where $\mathrm{E}_{k i n}$ is kinetic energy of the proton and $\mathrm{M}^{\prime}$ is given by,

$$
M^{\prime}=M_{n}-E_{B}
$$

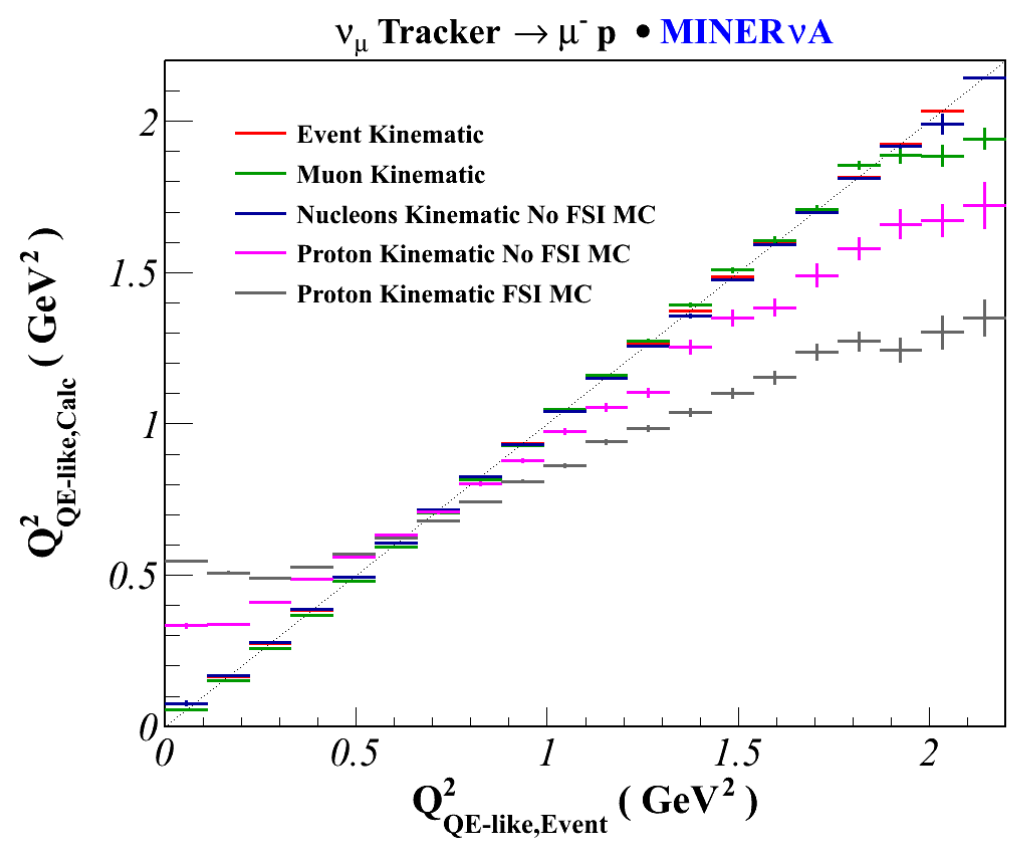

Figure 5.42. Plotted are the correlations between the $\mathrm{Q}^{2}$ calculated using the event(red), muon(green), nucleon(blue), and proton(magenta and light gray) kinematics as a function of $\mathrm{Q}^{2}$ calculated from the event kinematic. Note that these are $\nu_{\mu}$ QE-like events without any applied cuts. See text for full description of the plot. Plot is a courtesy of M. Betancourt of the MINER $\nu$ A Collaboration.

The text below describes the discrepancies that are observed between each of the curves in Figure 5.42, which shows the $\nu_{\mu}$ QE-like selection without any of the applied analysis requirements. These curves serve to demonstrate the correlations between the various $\mathrm{Q}^{2}$ calculations as function of the $\mathrm{Q}^{2}$ computed using the event kinematics (see Equation 5.5.3).

- The red profile histogram is produced using the Monte Carlo which includes final state interactions and the $\mathrm{Q}^{2}$ is calculated using Equation 5.5.3, which is insensitive to final state interactions. In addition, Equation 5.5.3 is a two-body 
system calculation. Recall that this curve includes both the resonant and DIS events that are QE-like, and these processes (for this selection) usually produce a lepton, nucleon, and pion at the scattering vertex. The remaining profile histograms in Figure 5.42 are interpreted with respect to this distribution.

- The $\mathrm{Q}^{2}$ that is calculated using the nucleon kinematic (blue profile histogram) is correlated with the $\mathrm{Q}^{2}$ calculated using the event kinematic. The nucleon kinematic distribution is produced with the simulation without FSI, and thus for this sample, the $\nu_{\mu}$ QE-like selection is composed only of the QE events. Therefore, the small discrepancy between the event and nucleon kinematic distributions, indicates that as $\mathrm{Q}^{2} \rightarrow \infty$ Equation 5.5.3 does not precisely model the $\mathrm{Q}^{2}$ of the resonance and DIS events that are QE-like.

- At higher values of $\mathrm{Q}^{2}$, the distribution which is calculated using the muon kinematic (uses Equation 5.5.4) abruptly drops from the diagonal line. This behavior also supports that $\mathrm{E}_{\nu}^{Q E}$ is inaccurate for calculating the neutrino energy for the resonance and DIS events that are observed as $\nu_{\mu}$ QE-like candidates.

- Both distributions which are produced using the proton kinematics (magenta and light gray profile histograms), are uncorrelated with the event kinematic $\mathrm{Q}^{2}$ at both lower and higher values of $\mathrm{Q}^{2}$. Foremost, the discrepancy between the magenta and light gray profiles is simply due to the effects of FSI. In addition, the magenta profile histogram that is calculated from the proton kinematic (see Equation 5.5.6) using the simulation without FSI, shows how Fermi motion can distorted the shape of the $\mathrm{Q}^{2}$ distribution.

Figure 5.43 illustrates an alternative interpretation of the $\mathrm{Q}^{2}$ correlations. The plot shows the correlations between the $\mathrm{Q}^{2}$ computed using the muon kinematic (see Equation 5.5.4) as function of the $\mathrm{Q}^{2}$ that is computed from the proton kinematic (see Equation 5.5.6) using both of the simulations with and without FSI. Fermi motion does smear the shape of the distribution. In the low $\mathrm{Q}^{2}$ regime, discrepancy is not as pronounced as the interpretation in Figure 5.42. As expected, the effects of FSI distorts the distribution in both the low and high $\mathrm{Q}^{2}$ region. In the low $\mathrm{Q}^{2}$ regime, the discrepancy is due to Fermi motion and the signal definition. Recall that the $\nu_{\mu}$ QE-like signal is defined as,

- one muon,

- no mesons,

- at least one proton, 


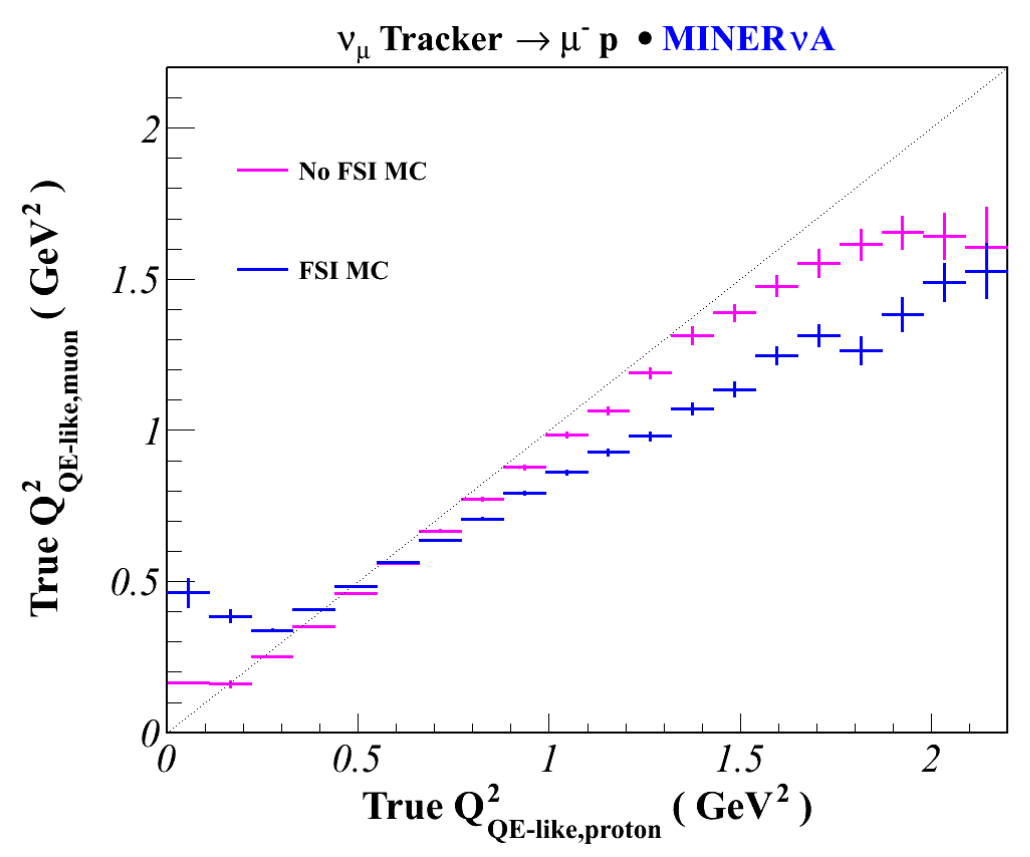

Figure 5.43. The correlations between the $\mathrm{Q}^{2}$ which is calculated using the muon and proton kinematics that are derived from the QE scattering. Plot is courtesy of M. Betancourt of the MINER $\nu$ A Collaboration.

in the final state. Note that there is not a condition on energy of the proton. There are cases when a proton with no kinetic energy is observed in the final state. These events populate the low $\mathrm{Q}^{2}$ region. In the high $\mathrm{Q}^{2}$ regime, the discrepancy is due to Fermi motion and the missing energy of the nucleons in the final state. The nuclear effects distort the shape of the $\mathrm{Q}^{2}$ distribution, particular for the $\mathrm{Q}_{\mathrm{QE} \text {,proton }}^{2}$ distribution. The next step is to examine how well does GENIE models these nuclear effects.

\subsubsection{THE MUON-PROTON KINEMATICS ANALYSIS}

The $\nu_{\mu}$ QE-like candidates with a muon track-matched to MINOS are selected for the muon-proton kinematic analysis. There are additional cuts applied to these events, which include the following:

1. The muon has a negatively charge. Recall that MINOS is able to determine the charge sign of tracks that propagate through it's detector. 
2. For these events, the neutrino energy is reconstructed from the muon kinematics assuming QE scattering, as indicated in Equation 2.3.1. The applied cut requires that the neutrino energy is reconstructed within the range of $1.5<\mathrm{E}_{\nu}<10$. GeV. The upper limit serves to remove events that are produced from the neutrinos in the high energy tail of the neutrino energy spectrum, which is less understood. The lower limit incorporates the MINOS track-matching energy acceptance.

These cuts are applied sequentially and have been extensively studied by the MINER $\nu \mathrm{A}$ QE analyses which reconstruct the event kinematics using only the muon. Figure 5.44 shows the reconstructed $\mathrm{Q}^{2}$ distributions using both the muon and proton kinematics.

These distributions illustrate that for the $\nu_{\mu}$ QE-like spectrum, that the Monte Carlo under-predicts the data. However, this underestimation is within the systematic uncertainties. In additional, the data prefers the Monte Carlo with FSI interactions (red histogram). This provides confidence in GENIE implementation of FSI, which is modeled by the intra-nuclear cascade model. For more examples of distributions which demonstrate GENIE modeling of FSI see Appendix B. 

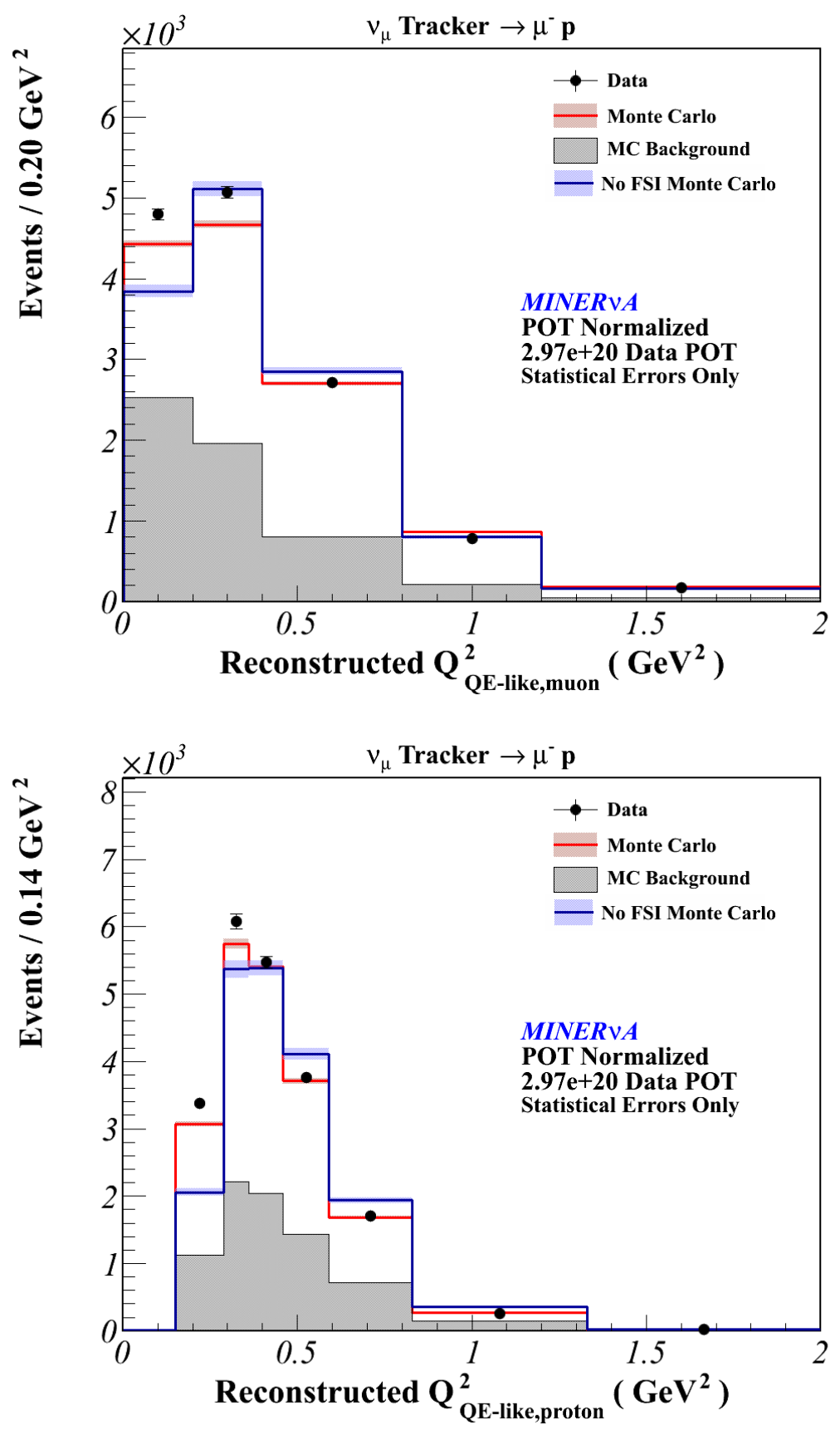

Figure 5.44. The absolutely comparisons of the reconstructed $\mathrm{Q}^{2}$ distributions using the muon (top) and proton (bottom) kinematics. The $\mathrm{Q}^{2}$ from the muon (which more accurately describe the true four-momentum transfer) shows that the backgrounds events populate the low $\mathrm{Q}^{2}$ regime. 


\section{SECTION 6}

\section{RESULTS AND CONCLUSIONS}

In Chapter 5, the measurement of the QE-like differential cross section using the proton kinematics is presented, along with the systematic uncertainties. The results show that GENIE underestimates the $\nu_{\mu}$ QE-like event rate. However, the discrepancy between the Monte Carlo and data is within the absolute systematic error bars on the data. The sources that contribute significantly to the total systematic uncertainty come from the GENIE modeling of the pion transportation in the nucleus and the resonance production cross section parameter, $\mathrm{M}_{A}$. Note that there exist MINER $\nu \mathrm{A}$ charged-current pion analyses that are in the process of measuring the resonance production $\mathrm{M}_{A}$ parameter and characterizing the effects of final state interactions. Therefore, the uncertainties on the GENIE sources will be reduced in the future. This chapter focuses on the interpretation of the $\nu_{\mu} \mathrm{d} \sigma / \mathrm{d} \mathrm{Q}_{\mathrm{QE}-\text { like,proton }}^{2}$ measurement that is extracted using the condition on the proton momentum, which requires at least one proton in the final state with momentum greater than $450 \mathrm{MeV} / \mathrm{c}$.

\subsection{COMPARING MINER $\nu \mathrm{A} \mathrm{d} \sigma / \mathrm{dQ}_{\mathrm{QE}}^{2}$ MEASUREMENTS}

There are a couple of validations which are performed to more clearly interpret

the $\mathrm{d} \sigma / \mathrm{d} \mathrm{Q}_{\mathrm{QE}-\text { like,proton }}^{2}$ results concerning the effects of final state interactions. These include:

- Comparing the QE component of the QE-like cross section with the MINER $\nu \mathrm{A}$ published QE cross section extracted from the inclusive muon sample (referred to as the one-track analysis).

- Comparing the $\mathrm{Q}^{2}$ reconstructed utilizing both the proton and muon kinematics, which was discussed in Subsection 5.5.2. 
In this section of the dissertation, the MINER $\nu \mathrm{A}$ published inclusive muon $\mathrm{QE}$ cross section measurement is referred to as the one-track QE analysis. This is because the event kinematics are measured using only the muon reconstructed observables (see Equation 5.5.4) and the proton track is not required to be reconstructed. Therefore, the one-track QE analysis selects only events with a muon track-matched by the MINOS detector. The one-track QE analysis is able to efficiently reconstruct QE events in the low $\mathrm{Q}^{2}$ regime, because it does not depend on the proton track reconstruction and the corresponding $\mathrm{Q}^{2}$ threshold.

In this section, the extracted pure QE component of the QE-like signal is referred to as the $n$-track QE analysis, because the protons are identified and track reconstructed. To compare the one-track and the n-track QE cross section measurements, only the MINOS-matched component of the $n$-track QE candidates is utilized, where the event $\mathrm{Q}_{\mathrm{QE} \text {,muon }}^{2}$ is reconstructed using the muon kinematics. The primary differences between the two types of QE analysis are:

1. the treatment of the recoil system in the event selection, and

2. the data-driven technique that is used for tuning the non-QE backgrounds.

In spite of these distinctions, the comparison between the two measurements is very important because the QE cross sections that are produced using the muon observables, are less sensitive to final state interactions of the proton propagation through the nucleus. Therefore, the comparison between the one-track and n-track QE cross sections can provide critical information concerning how well GENIE models the proton transportation. Due to FSI effects, the scattered proton's direction may abruptly change. Only the cross sections in the $\mathrm{Q}_{\mathrm{QE} \text {,muon }}^{2}$ bins that have measurements from both QE analyses are compared.

Figure 6.1 shows the measured cross sections from the one-track and $n$-track analyses and Table 6.1 gives the numerical values per $\mathrm{Q}_{\mathrm{QE} \text {,muon }}^{2}$ bin. The ratio between the 


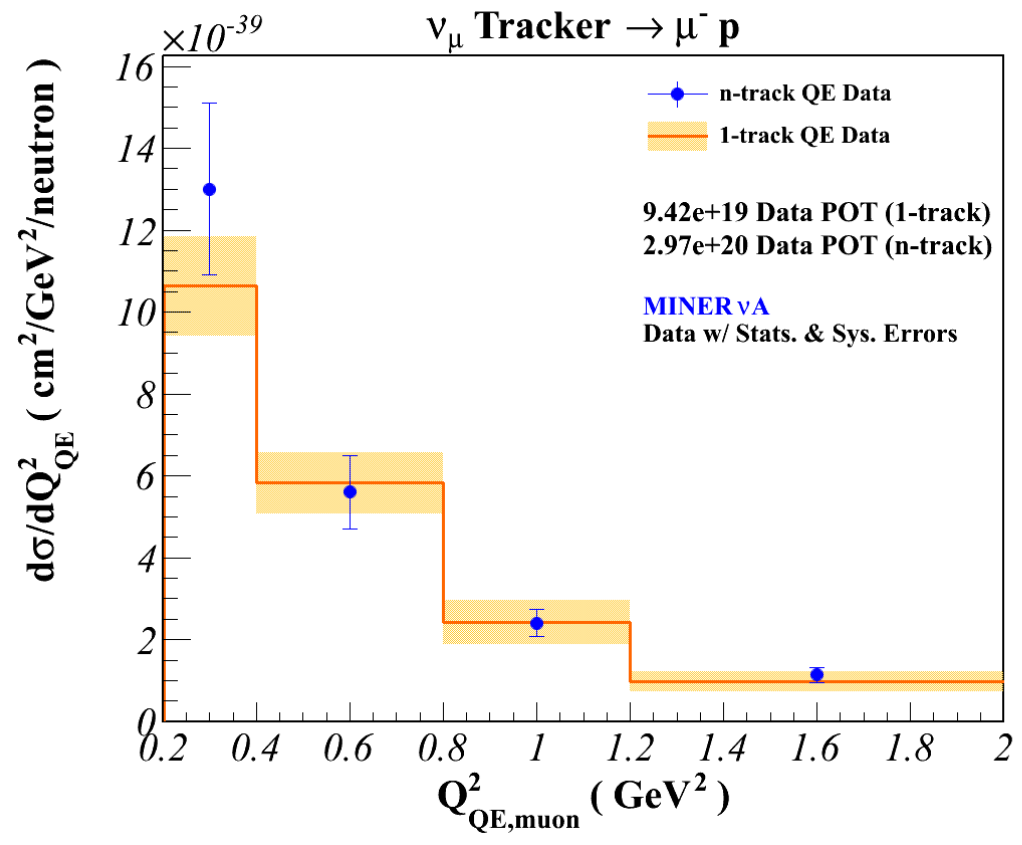

Figure 6.1. The absolutely normalized one-track (orange) and n-track (blue) QE differential cross sections comparison. The one-track measurements are provided by Reference [1].

Table 6.1. Flux-integrated $\mathrm{d} \sigma / \mathrm{dQ}_{\mathrm{QE}}^{2}$ Summary for the inclusive and exclusive $\mathrm{QE}$ analyses. Both the statistical (first column) and systematic (second column) errors are presented for each measurement. The one-track cross section is reprinted from Reference [1]

\begin{tabular}{c|cc}
\hline $\begin{array}{c}\mathrm{Q}_{\mathrm{QE}, \text { muon }}^{2} \\
\left(\mathrm{GeV}^{2}\right)\end{array}$ & $\begin{array}{c}\text { Cross-section (one-track) } \\
\left(10^{-38} \mathrm{~cm}^{2} / \mathrm{GeV}^{2} / \text { neutron }\right)\end{array}$ & $\begin{array}{c}\text { Cross-section }(\text { n-track }) \\
\left(10^{-38} \mathrm{~cm}^{2} / \mathrm{GeV}^{2} / \text { neutron }\right)\end{array}$ \\
\hline $0.2-0.4$ & $1.063 \pm 0.019 \pm 0.120$ & $1.301 \pm 0.036 \pm 0.206$ \\
$0.4-0.8$ & $0.582 \pm 0.013 \pm 0.074$ & $0.561 \pm 0.013 \pm 0.088$ \\
$0.8-1.2$ & $0.242 \pm 0.014 \pm 0.053$ & $0.240 \pm 0.012 \pm 0.031$ \\
$1.2-2.0$ & $0.097 \pm 0.008 \pm 0.024$ & $0.114 \pm 0.012 \pm 0.014$ \\
\hline
\end{tabular}


$\mathrm{d} \sigma / \mathrm{dQ}_{\mathrm{QE}}^{2}$ is presented in Figure 6.2. Note that in the Figures 6.1 and 6.2 that about a third of the events are correlated between the n-track and one-track QE analyses, and the statistical errors have not been separated into correlated and uncorrelated components. The $n$-track measurements are produced with a cut placed on the neutrino energy $\left(1.5<\mathrm{E}_{\nu}<10.0 \mathrm{GeV}\right)$ for consistency with the one-track analysis [1]. The calculated neutrino integrated-flux between the range of 1.5 and $10.0 \mathrm{GeV}$ is $\Phi=2.91 \times 10^{-8} / \mathrm{cm}^{2}$ and $2.78 \times 10^{-8} / \mathrm{cm}^{2}$ per P.O.T for the one-track and $n$-track QE analyses, respectively. The calculated number of neutrons in the MINER $\nu \mathrm{A}$ standard fiducial volume for the one-track and n-track is $\mathrm{T}=1.65 \times 10^{30}$ and $\mathrm{T}=1.53 \times 10^{30}$, respectively. For the one-track QE analysis, the values for the mass fractions of the scintillator plane were $\sim 1$ incorrect [104]. The one-track (n-track) has $\sim 65 \%(\sim 20 \%)$ purity at low $\mathrm{Q}_{\mathrm{QE}, \text { muon }}^{2}$ and $\sim 35 \%(\sim 80 \%)$ in the high $\mathrm{Q}_{\mathrm{QE}, \text { muon }}^{2}$ regime, where the resonant production is the dominate background in each. Since the analyses have a different fraction of background to signal in each $\mathrm{Q}_{\mathrm{QE} \text {,muon }}^{2}$ bin, it is likely that the primary discrepancy comes from the background subtraction of the resonant events. Since the $n$-track QE analysis requires that the proton is tracked, the proton tracking efficiency may also accounts for some part of the discrepancy that exists between the data and Monte Carlo. As mentioned in Subsection 5.3.3, the efficiency function in the $n$-track analysis does not account for any proton tracking discrepancies that may exist between the Monte Carlo and data. Based on preliminary studies of scanning two-track events, this discrepancy is expected to be small.

Table 6.1 shows which systematic uncertainties are included for each QE analysis. In this dissertation, the one-track and n-track QE cross sections are produced using different versions of the MINER $\nu \mathrm{A}$ analysis framework. Therefore, the common systematic sources, such as the neutrino flux, have not been factored out and are included in the systematic errors on both the one-track and n-track QE cross sections (see Figures 6.1 and 6.2 ). Table 6.1 shows that for the ratio of the one-track to $n$ - 
track, the systematic uncertainty on the neutrino flux are nearly identical, since it is correlated between the analyses. In the near-future when the one-track cross section is re-produced, the muon reconstruction systematic uncertainty and various GENIE contributions of the primary neutrino interactions and final state interactions will be correctly canceled. Recall that the events are isolated differently between analyes, therefore not all of the GENIE systematic uncertainties will cancel.

The ratio plot in Figure 6.2 shows that the cross section results are consistent for all bins of $\mathrm{Q}_{\mathrm{QE} \text {,muon }}^{2}$, except for the first bin. For the $n$-track $\mathrm{QE}$ analysis, the purity

is the worst for $\mathrm{Q}_{\mathrm{QE} \text {, muon }}^{2}$ values less than $0.4 \mathrm{GeV}^{2}$. This provides confidence in the treatment of the recoil system for the $n$-track QE event selection, particularly since the n-track QE analysis is more sensitive to the effects of final state interactions. The cross section results from the one-track QE analysis agree best with the RFG model that includes the TEM and illustrate that the MINER $\nu \mathrm{A}$ neutrino-nucleus QE scattering data is sensitive to the knockout of multinucleons at the scattering vertex due to meson exchange currents (see Figure 6.3). This implies that the $\nu_{\mu}$ QE-like analysis should also be sensitive to such contributions, because the results are consistent between the one-track and n-track QE analyses, where the n-track QE data is a subset of the QE-like data.

\subsection{MODEL COMPARISONS OF THE MEASURED d $\sigma / \mathrm{dQ}_{\mathrm{QE}-\text { like,proton }}^{2}$}

The measured $\nu_{\mu} \mathrm{d} \sigma / \mathrm{dQ}_{\mathrm{QE}-\text { like,proton }}^{2}$ is also compared with models other than GENIE. The neutrino event generator models which are compared with the data were produced by J. Sobczyk using the Neutrino Event Generator developed by the Wroclaw Neutrino Group (NuWro) [105]. The NuWro models which are used to describe the nuclear structure and the scattering physics of the QE component of the $\nu_{\mu}$ QE-like channel includes one of the following: 


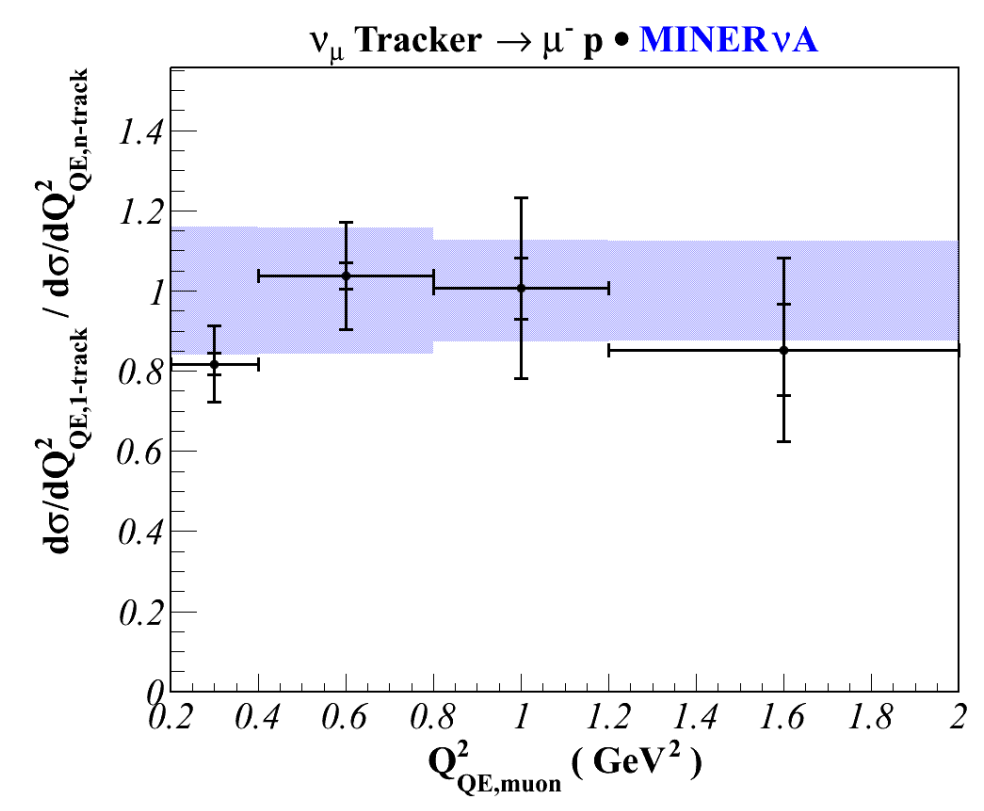

Figure 6.2. Ratio between the absolutely normalized one-track and n-track QE differential cross sections. The blue error band represents the total absolute systematic uncertainty on the $n$-track QE cross section. The inner errors bars on the data points show the total statistical errors for both analyses and the outer error bars show the total absolute systematic errors for the one-track QE cross section, respectively.

Table 6.2. The fractional systematic uncertainties on the one-track (top) and n-track (bottom) $\mathrm{QE}$ cross section measurements for each $\mathrm{Q}_{\mathrm{QE} \text {,muon }}^{2}$ bin. The contributors are the (I) flux, (II) neutrino interaction models, (III) final state interaction models, (IV) muon reconstruction (V) recoil reconstruction, (VI) other. The one-track uncertainties are reprinted from Reference [1].

\begin{tabular}{|c||cccccc|}
\hline \hline \multicolumn{7}{|c|}{ one-track } \\
\hline $\mathrm{Q}_{\mathrm{QE}, \text { muon }}^{2}\left(\mathrm{GeV}^{2}\right)$ & $\mathrm{I}$ & $\mathrm{II}$ & III & IV & V & VI \\
\hline $0.2-0.4$ & 0.09 & 0.03 & 0.03 & 0.05 & 0.02 & 0.01 \\
$0.4-0.8$ & 0.09 & 0.04 & 0.04 & 0.05 & 0.03 & 0.01 \\
$0.8-1.2$ & 0.09 & 0.07 & 0.15 & 0.08 & 0.07 & 0.02 \\
$1.2-2.0$ & 0.09 & 0.07 & 0.16 & 0.12 & 0.07 & 0.02 \\
\hline \multicolumn{7}{|c|}{$n$-track } \\
\hline $0.2-0.4$ & 0.09 & 0.04 & 0.12 & 0.04 & 0.03 & 0.01 \\
$0.4-0.8$ & 0.09 & 0.06 & 0.11 & 0.04 & 0.03 & 0.03 \\
$0.8-1.2$ & 0.08 & 0.06 & 0.07 & 0.04 & 0.01 & 0.01 \\
$1.2-2.0$ & 0.08 & 0.08 & 0.04 & 0.04 & 0.02 & 0. \\
\hline
\end{tabular}




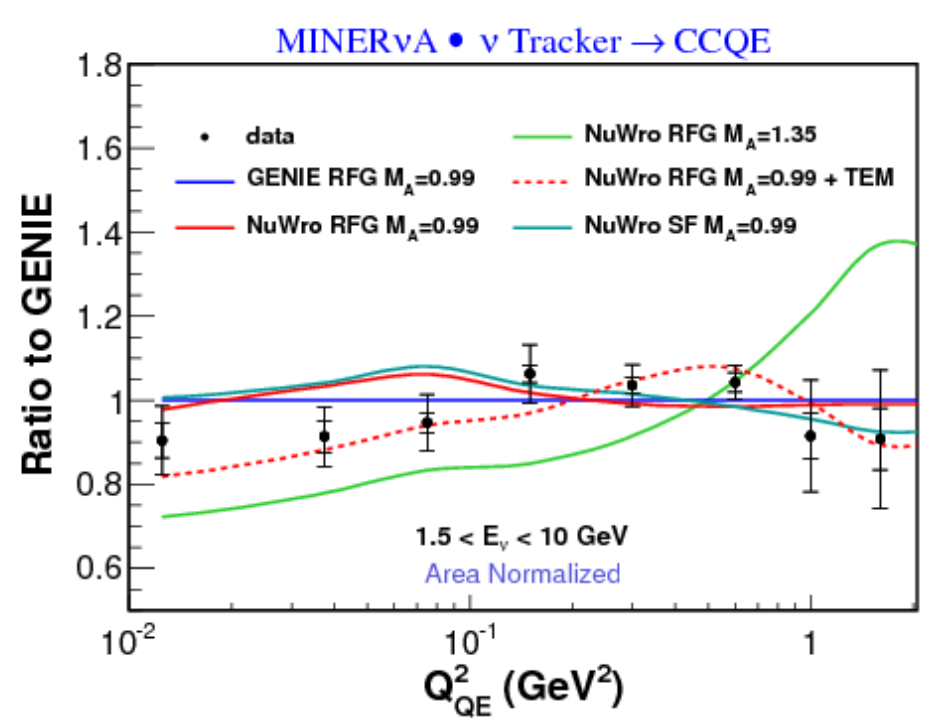

Figure 6.3. The one-track QE data and model comparison. The data prefers the RFG+TEM (dashed line) model which takes into account the enhancement in the cross section due to the knockout of multinucleons at the scattering vertex. Plot is courtesy of L. Fields of the MINER $\nu$ A Collaboration.

- Relativistic Fermi Gas model (RFG), $\mathrm{M}_{A}=0.99 \mathrm{GeV}$

- Local Fermi Gas model (LFG), $\mathrm{M}_{A}=0.99 \mathrm{GeV}$.

The QE process in NuWro is computed with the standard formalism of LlewellynSmith [21] which models the QE cross section using the plane wave impulse approximation to describe neutrino scattering. In addition, NuWro has several options for parametrizations describing the vector form factors [50], where the BBBA Form Factors [26] are utilized in the NuWro predictions presented in this dissertation. For modeling the nuclear dynamics that leads to multinucleon knockouts, NuWro has an implementation of the following models:

- Nieves-Meson Exchange Currents (MEC) is a microscopic model and treated as a separate interaction process [106].

- Transverse Enhancement Model (TEM) is an effective model that is developed based on inclusive electron-nucleus scattering data (see Subsection 2.3.2). It is implemented as an independent interaction process, where the event kinematics for these events are not treated as a two-body system [106]. 
Recall that both resonance production and DIS contributes to the $\nu_{\mu}$ QE-like cross sections. NuWro defines the resonance production as $W<1.6 \mathrm{GeV}$, and the events with $W>1.6 \mathrm{GeV}$ are defined as the DIS production. Unlike the GENIE implementation of the higher resonances, NuWro utilizes the quark-hadron duality hypothesis to describe the average cross section from higher excited resonance states. The DIS reaction is evaluated using the Bodek-Yang prescription.

The NuWro $\nu_{\mu} \mathrm{d} \sigma / \mathrm{dQ}_{\mathrm{QE}-\text { like,proton }}^{2}$ predictions are produced by generating neutrino scattering events on a pure Carbon target. Due to the lepton flavor conservation, neutrinos can only interact with neutrons in the quasielastic process. Thus, the neutrino cannot quasi-elastically scatter from a Hydrogen nucleus. Since the Hydrogen atom consists of a single proton, the ejected hadrons from the resonance and DIS productions do not experience any effects due to final state interactions. Therefore, the pions that are produced in these interactions, are observed in the final state, where such events do not satisfy the QE-like signal definition. The following list gives the various combinations of the NuWro cross sections which are used to compare with the data:

- NuWro RFG, $\mathbf{M}_{A}=\mathbf{0 . 9 9}$ with the Resonant and DIS QE-like components,

- NuWro RFG, $\mathbf{M}_{A}=\mathbf{0 . 9 9}+$ TEM with the Resonant and DIS QE-like components,

- NuWro LFG, $\mathbf{M}_{A}=\mathbf{0 . 9 9}+$ Nieves-MEC with the Resonant and DIS QElike components.

To interpret the measured $\nu_{\mu} \mathrm{d} \sigma / \mathrm{dQ}_{\mathrm{QE}-\text { like,proton }}^{2}$ (see Figure 5.33), the next Subsection focuses on three aspects of the analysis, which include the following:

1. A comparison of the models implemented for the various components of the QE-like cross section,

2. The GENIE modeling of the Resonant production,

3. The cross section shape analysis. 


\subsubsection{INTERPRETING THE $\mathrm{d} \sigma / \mathrm{dQ}_{\mathrm{QE}-\text { like,proton }}^{2}$ MEASURE- MENT}

\section{Comparing GENIE and NuWro Models}

The comparison between the GENIE and NuWro modeling of the pure QE cross section and the effects of the nuclear medium are presented in Figure 6.4, with the ratio of each model to GENIE RFG shown in Figure 6.5. The differences between the models are less than $5 \%$ for $\mathrm{Q}_{\mathrm{QE} \text {,proton }}^{2}<1.5 \mathrm{GeV}^{2}$ and $10-15 \%$ for $\mathrm{Q}_{\mathrm{QE} \text {,proton }}^{2}$ $>1.5 \mathrm{GeV}^{2}$. The initial state nucleons that are described by the NuWro RFG model have a momentum distribution based solely on the Fermi Gas model, which requires that the nucleon's momentum is not greater than the Fermi momentum in the Carbon nucleus. Recall that GENIE models the high momentum tail of the momentum distribution of the nucleons due to nucleon-nucleon SRC using the Bodek-Richie prescription [107]. The NuWro LFG models the nucleons with a mean effective potential.

Although the momentum spectrum of the initial state nucleons differs for each model, the total integral of each distribution is the same. Therefore, the small disagreement between the models in the low to moderate $\mathrm{Q}_{\mathrm{QE} \text {,proton }}^{2}$ regime is most likely due to the shape of the momentum distribution of the initial state nucleons. Recall that the proton can inelastically scatter from a spectator nucleon before exiting the nucleus and this collision can produce additional nucleons and pions. Thus, the dis-

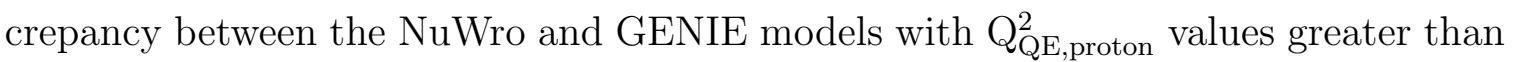
$1.5 \mathrm{GeV}^{2}$, is most likely to be due to the modeling of the proton transportation in the nucleus.

As described earlier, GENIE and NuWro define the resonant and DIS productions, differently. Figure 6.6 shows the comparison between the sum of the resonant and DIS cross sections for GENIE and NuWro. Only the GENIE inelastic cross section includes the modeling of Formation Time (FT) effects. Recall that FT can be related to a fundamental QCD phenomenon known as color transparency (CT), the production 


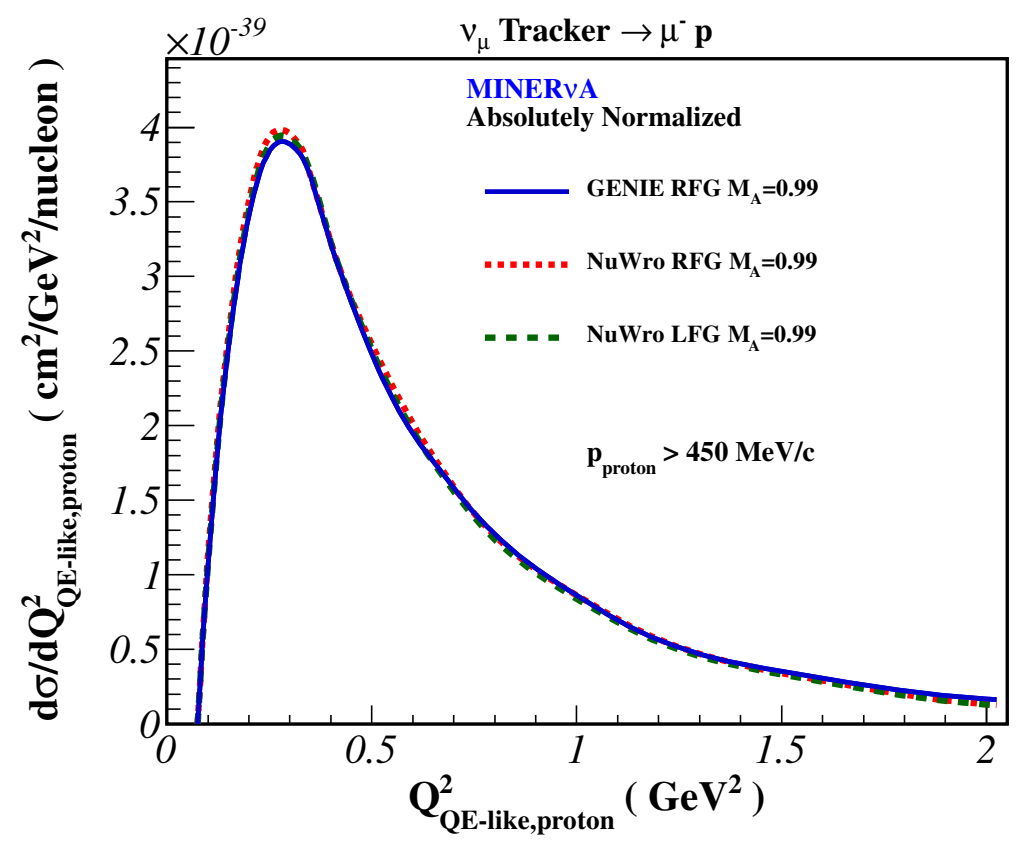

Figure 6.4. GENIE RFG (blue), NuWro RFG (red), and NuWro LFG (green) predictions of $\mathrm{QE}$ component of the QE-like cross section, with at least one proton having momentum greater than $450 \mathrm{MeV} / \mathrm{c}$ in the final state.

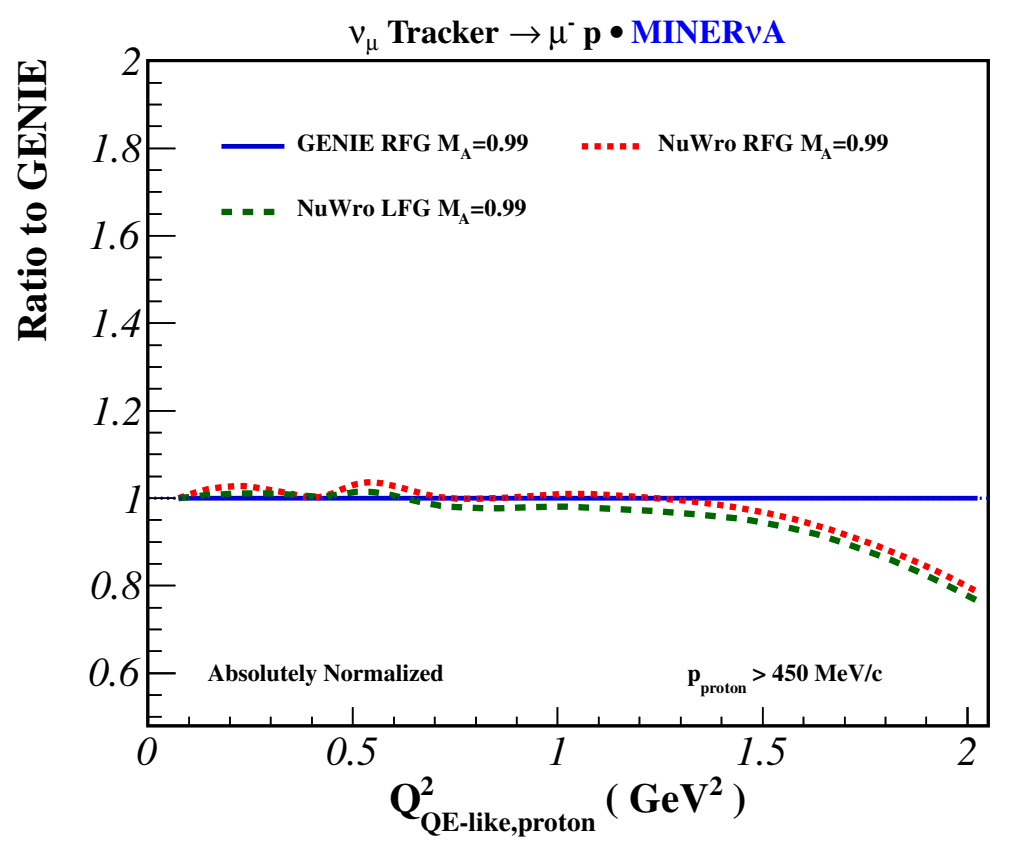

Figure 6.5. Ratio of the GENIE RFG (blue), NuWro RFG (red), and NuWro LFG (green) to GENIE RFG for the curves plotted in Figure 6.4. 
time and formation length it takes for the quarks to materialize into hadrons [50]. It is predicted that the two-quark systems are more probable to create these point like configurations than the three-quark systems. Therefore, the CT effects are expected to be greater for pions than nucleons. In the Monte Carlo neutrino event generators, the strong interaction is suppressed until the hadron (point like configuration) is formed [50]. Thus, there is a greater probability that the hadron exits the nucleus without experiencing any effects from final state interactions [50]. In spite of the FT effects, overall GENIE predicts a larger contribution for the inelastic component of the QE-like cross section than NuWro, with the current studies indicating that the discrepancy between the GENIE and NuWro cross sections is due primarily to the modeling of the pion production cross section and the pion propagation in the nucleus.

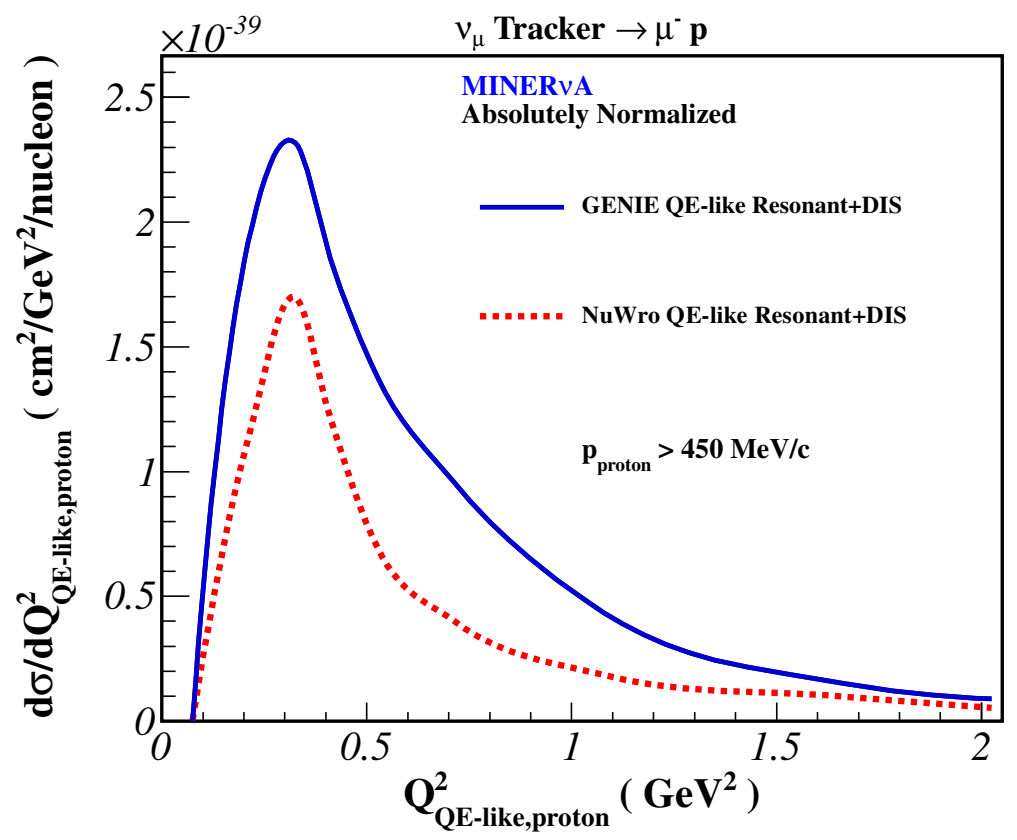

Figure 6.6. GENIE (solid line) and NuWro (dashed line) comparison of the inelastic component of the QE-like cross section. 


\section{GENIE Modeling of the Resonant Production}

Recall that the data driven two-component background tuning procedure (see Subsection 5.3.1) resulted in the resonant component of the QE-like background being scaled down by approximately $50 \%$ (see Figure 5.21). In addition, the cross section results from the MINER $\nu \mathrm{A}$ single $\mathrm{CC} \nu_{\mu}$ inclusive pion analysis also show that GENIE overestimates the pion production event rate, as indicated in Figure 6.7 [89]. Both the $\nu_{\mu}$ QE-like and $\nu_{\mu}$ single-pion analyses, which are independent analyses, provide evidence which suggest that GENIE mis-models the pion production reaction. This is critical information and must be taken into account before interpreting the $\nu_{\mu}$ $\mathrm{d} \sigma / \mathrm{dQ}_{\mathrm{QE}-\text { like,proton }}^{2}$ results. The additional strength that is observed in the GENIE resonant component of the QE-like cross section (see Figure 6.6) can lead to misinterpetations concerning the comparison to data.

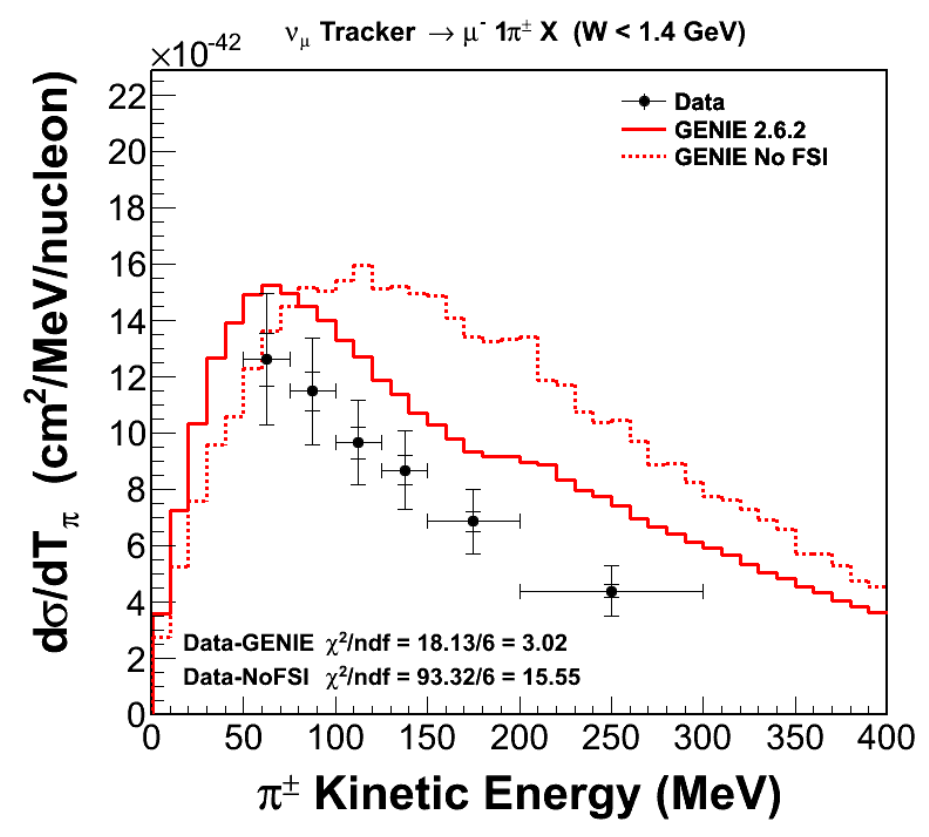

Figure 6.7. Comparison of the absolutely normalized data and Monte Carlo with (solid line) and without (dashed line) FSI effects for the charged-current single pion production cross section as a function of the pion kinetic energy. Plot courtesy of B. Eberly of the MINER $\nu$ A Collaboration. 
Before comparing the extracted cross section measurements to the various neutrino Monte Carlo event generators, the resonant component of the GENIE QE-like cross section is scaled down by $30 \%$ (see Figure 6.8), which is based on the results of the charged-current single pion analysis. As a result, the GENIE and NuWro predic-

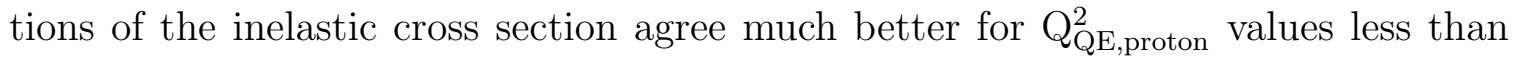
$0.5 \mathrm{GeV}^{2}$. However, there is still a 20-50\% discrepancy between the inelastic cross

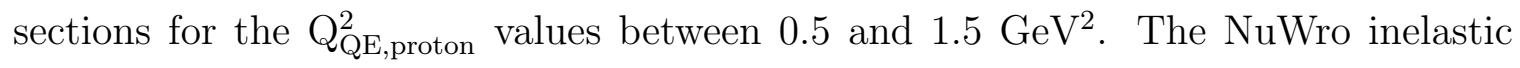
cross section drops much more rapidly with $\mathrm{Q}_{\mathrm{QE} \text {,proton }}^{2}$ than GENIE cross section in this region. NuWro and GENIE modeling of the pion absorption cross section within their INC framework are most likely responsible for the disagreement [89]. These inconsistent predictions lead to different interpretations about the data. Therefore, it becomes difficult to disentangle the mis-modeling of the quasielastic scattering from the inelastic scattering for the QE-like cross section.

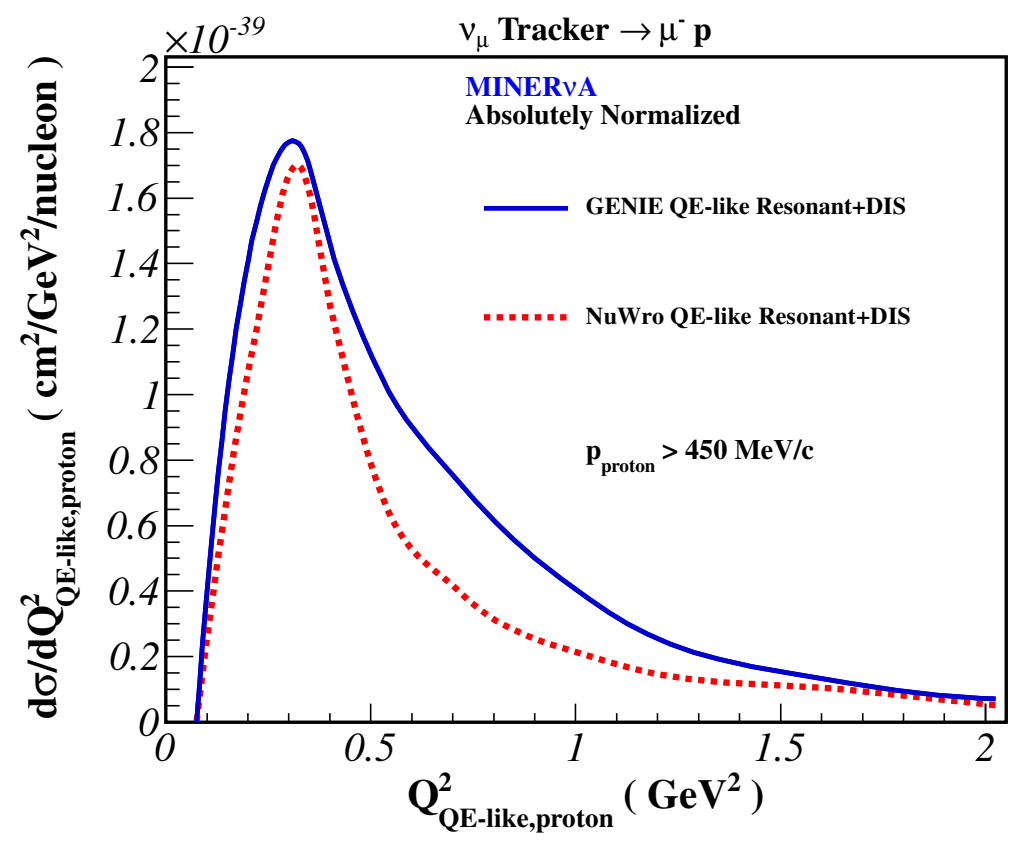

Figure 6.8. GENIE (solid line) and NuWro (dashed line) inelastic components of the QElike cross section, where the GENIE resonant component has been scaled down by $30 \%$. 

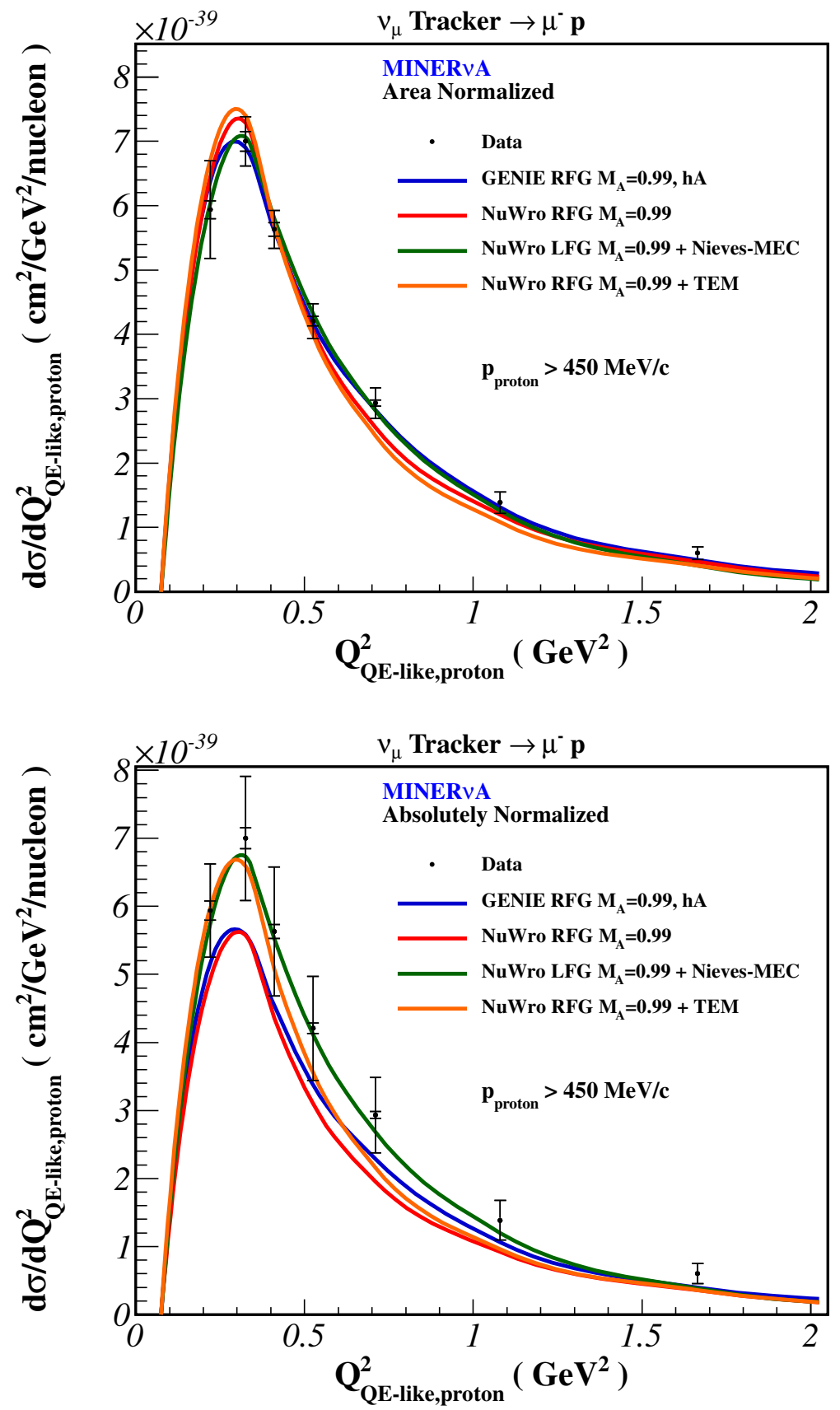

Figure 6.9. Plotted are the absolute (top) and shape (bottom) comparisons of the models to the data. The GENIE resonant component of the QE-like cross section has been scaled down by $30 \%$. 
Table 6.3. Calculated $\chi^{2}$ between the measured $\mathrm{d} \sigma / \mathrm{d} \mathrm{Q}_{\mathrm{QE}-\text { like,proton }}^{2}$ and various models with $\mathrm{M}_{A}=0.99 \mathrm{GeV} / \mathrm{c}^{2}$ and the $\mathrm{p}_{\text {proton }}>450 \mathrm{MeV} / \mathrm{c}$. The degrees of freedom, d.o.f is 7 (6) for the rate (shape) $\chi^{2}$ calculation.

\begin{tabular}{l||c|c}
\hline \hline Model & Rate $\chi^{2} /$ d.o.f & Shape $\chi^{2} /$ d.o.f \\
\hline \hline GENIE RFG & 0.72 & 0.83 \\
NuWro RFG & 0.96 & 1.28 \\
NuWro RFG + TEM & 1.64 & 2.07 \\
NuWro LFG + MEC & 1.55 & 2.39 \\
\hline
\end{tabular}

\section{The $\mathbf{d} \sigma / \mathbf{d Q}_{\mathrm{QE}-\text { like,proton }}^{2}$ Shape Analysis}

Figure 6.9 shows the comparison of the models to the data and Table 6.3 gives the calculated $\chi^{2}$ between the data and models, which is given by,

$$
\chi^{2}=\sum_{i} \sum_{j}\left(N_{i}^{\text {data }}-N_{i}^{\text {model }}\right) C_{i j}\left(N_{j}^{\text {data }}-N_{j}^{\text {model }}\right),
$$

where $\mathrm{C}_{i j}$ is the data covariance matrix element in bin $i j$. For the shape analysis (the top plot shown Figure 6.9), the models are area normalized to the data, where the normalization constants are given in Table 6.4. Before discussing the results of the calculated $\chi^{2}$, the characteristic features of the models are summarized below.

- The resonant component of the GENIE QE-like cross section has been scaled down by $30 \%$. For the absolute normalized distribution (bottom plot) in Figure 6.9, the GENIE RFG and NuWro RFG distributions agree in the peak. However, for the area normalized distribution (top plot), the GENIE RFG and $\mathrm{NuWro} \mathrm{RFG} \mathrm{predictions} \mathrm{disagree} \mathrm{in} \mathrm{the} \mathrm{peak,} \mathrm{which} \mathrm{is} \mathrm{due} \mathrm{very} \mathrm{different} \mathrm{pre-}$ dictions of the inelastic component of the QE-like cross section between NuWro

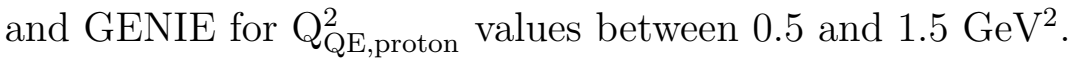

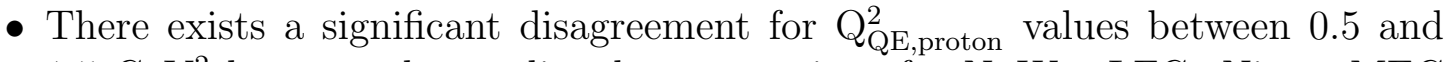
1.5 $\mathrm{GeV}^{2}$ between the predicted cross sections for NuWro LFG+Nieves-MEC and NuWro RFG+TEM, where both curves include the same NuWro prediction of the inelastic component of the QE-like cross section. Recall that Figure 6.5 shows that there is only a small disagreement between NuWro LFG and NuWro RFG pure QE component. Therefore, the larger part of the discrepancy between the NuWro LFG+Nieves-MEC and NuWro RFG+TEM comes from the

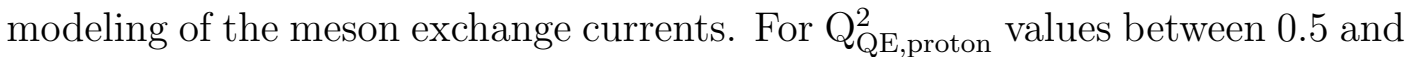
$1.5 \mathrm{GeV}^{2}$, the Nieves-MEC predicts that 2particle 2hole contributes more to the 
cross section than the does TEM prediction. The additional strength that is produced from Nieves-MEC modeling of the 2particle 2hole compenstates for the loss of strength that is modeled by the NuWro inelastic component of the QE-like cross section in the $\mathrm{Q}_{\mathrm{QE} \text {,proton }}^{2}$ region of 0.5 to $1.5 \mathrm{GeV}^{2}$.

The total systematic uncertainty on the measured $\mathrm{d} \sigma / \mathrm{dQ}_{\mathrm{QE}-\text { like,proton }}^{2}$ is reduced in the shape analysis, as indicated by Figure 6.9. Section 5.4 summarizes the calculation of the shape errors, which are designed to assign a systematic uncertainty on the shape of the distribution. The systematic uncertainty on the neutrino flux is negligible (see Figure 5.34) in the shape analysis. In addition, the absolute errors on the GENIE modeling of the primary neutrino interaction reduces by $\sim 50 \%$ relative to the shape errors. The primary contributors to the total systematic uncertainty are the GENIE INC modeling of the pion inelastic and absorption cross sections.

Based on the calculated $\chi^{2}$ (see Table 6.3), the GENIE RFG with the inelastic component best describes the data. However, the last $\mathrm{Q}_{\mathrm{QE} \text {,proton }}^{2}$ bin $\left(\mathrm{Q}_{\mathrm{QE} \text {,proton }}^{2}\right.$ $\geq 1.5 \mathrm{GeV}^{2}$ ) primarily drives the calculated $\chi^{2}$ values between the data and models. As shown in Figure 6.5, GENIE predicts a larger contribution from the QE component of the $\mathrm{d} \sigma / \mathrm{dQ}_{\mathrm{QE}-\text { like,proton }}^{2}$ than does NuWro. The disagreement between the

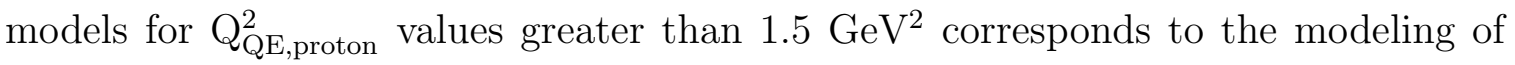
the proton propagation in the nucleus.

If the last $\mathrm{Q}_{\mathrm{QE} \text {,proton }}^{2}$ bin is excluded from the $\chi^{2}$ calculation, then NuWro LFG+NievesMEC with the inelastic component best describes the data. This interpretation also introduces many puzzles. The Nieves-MEC modeling of the 2particle 2hole compensates for the weaker strength that is modeled by NuWro inelastic component of the QE-like cross section for $\mathrm{Q}_{\mathrm{QE} \text {,proton }}^{2}$ values between 0.5 and $1.5 \mathrm{GeV}^{2}$. Recall that in this region, NuWro modeling of the inelastic component of the QE-like cross section is smaller than the GENIE modeling (see Figure 6.8). In addition, the shape between the MINER $\nu \mathrm{A}$ pion production data and GENIE model agrees fairly well for pions with kinetic energy values between 100 and $200 \mathrm{MeV}$, as shown in Figure 6.10. This 
could indicate that GENIE does fairly well for modeling the shape of the inelastic component of the cross section. However, this interpretation is inconsistent with the results from the QE-like data, which prefers NuWro LFG+Nieves-MEC prediction that include an inelastic component with a different shape than GENIE. Therefore, it becomes difficult to disentangle which component of the QE-like cross section is inaccurately modeled, meson exchange currents or inelastic processes with no pion in the final state from this data set alone.

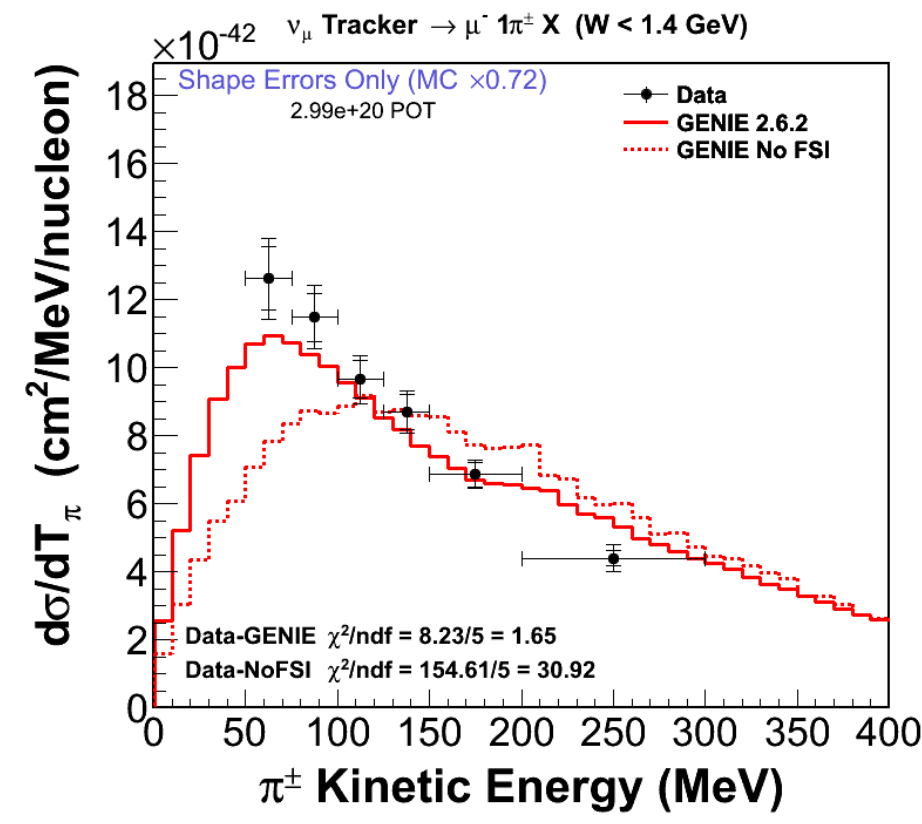

Figure 6.10. Comparison of the area normalized data and Monte Carlo with (solid line) and without (dashed line) FSI effects for the charged-current single pion production cross section as a function of the pion kinetic energy. Plot courtesy of B. Eberly of the MINER $\nu$ A Collaboration.

Table 6.4. The model to the measured data $\mathrm{d} \sigma / \mathrm{dQ}_{\mathrm{QE}-\text { like,proton }}^{2}$ area normalization constant.

\begin{tabular}{l||c}
\hline \hline Model & Area Normalization Constant \\
\hline \hline GENIE RFG & 1.24 \\
NuWro RFG & 1.31 \\
NuWro RFG + TEM & 1.12 \\
NuWro LFG + MEC & 1.05 \\
\hline
\end{tabular}




\subsection{THEORETICAL PREDICTIONS FOR EXAMINING THE EFFECTS OF FSI}

As mentioned earlier, the intra-nuclear cascade models are validated by comparing their results with the hadron-nucleus scattering data. In electron scattering, the final state interaction models can be checked aganist nuclear transparency measurements [108]. The nuclear transparency is a measure of the probability that the struck proton with a particular energy will escape the nucleus undistrubed. In addition, nuclear transparency measurements are useful for studying the nuclear medium, particularly color transparency [108], which was briefly discussed earlier. The definition of transparency that is used by electron-nucleus scattering experiments is given by,

$$
T\left(Q^{2}\right)=\frac{\int_{v} d^{3} p_{m} d E_{m} Y_{e x p}\left(E_{m}, \vec{p}_{m}\right)}{\int_{v} d^{3} p_{m} d E_{m} Y_{P W I A}\left(E_{m}, \vec{p}_{m}\right)},
$$

where $\mathrm{E}_{m}$ and $\left|\mathrm{p}_{m}\right|$ is the missing energy and momentum of the struck proton. The terms, $\mathrm{Y}_{\exp }$ and $\mathrm{Y}_{P W I A}$ are the measured and theoretical calculated yield of events that have a proton that does not undergo any FSI effects. The electron scattering experiments evaluated the nuclear transparencies in the QE regime by analyzing coincidence measurements. There are models which describe the nuclear transparencies for the electron-nucleus scattering, such as the relativistic multiple-scattering Glauber approximation (RMSG) [108], which more accurately describes the final state interactions for $\mathrm{Q}^{2} \geq 0.5 \mathrm{GeV}^{2}$ than the intra-nuclear cascade models [109]. Since the hadron propagation through the nucleus is the same in both the electron and neutrino scattering, it would be helpful for the neutrino scattering event generators to both incorporate and validate different treatments of the final state interactions in the future. This will provide an alternative interpretation for describing the final state interactions. 


\subsection{CONCLUSIONS}

The $\mathrm{d} \sigma / \mathrm{dQ}_{\mathrm{QE}-\text { like,proton }}^{2}$ measurement on the MINER $\nu \mathrm{A}$ hydrocarbon target is presented in this dissertation. This analysis requires that the muon and at least one proton are detected. Due to the poor momentum resolution for the muons that are not matched to tracks in MINOS, the $\mathrm{Q}_{\mathrm{QE} \text {,proton }}^{2}$ is reconstructed using only the leading proton kinematics. In addition, the tracking threshold prevents the reconstruction of events at low $\mathrm{Q}^{2}$. In spite of the reconstruction inefficiencies, this analysis displays very interesting results.

The QE-like differential cross section is measured as a function of $\mathrm{Q}_{\mathrm{QE} \text {,proton }}^{2}$, which is calculated assuming QE scattering from a free nucleon at rest. The measured cross section is distorted in $\mathrm{Q}_{\mathrm{QE} \text {,proton }}^{2}$ because of the Fermi motion of the initial state nucleons and the final state interaction effects. The variables that are sensitive to the modeling of FSI effects, such as the difference between the $\mathrm{Q}^{2}$ reconstruced from the muon and proton observables, are used to evaluate GENIE modeling of the proton transportation through the nucleus. The MINOS-matched component of the QE-like selection is utilized to reconstruct these variables, which require information from both the muon and leading proton, which is sensitive to the effects of FSI. Figures in Subsection 5.5.2 and Appendix B illustrate that there is resonable agreement between the simulation and data for variables which includes both the muon and leading proton reconstructed observables. These results provide confidence in the GENIE modeling of the leading proton transportation through the nucleus and could be utilized for further studies in the future, such as quantifying the energy loss in the nucleus by the final state proton.

The extracted pure QE component of the $\nu_{\mu}$ QE-like cross section is compared with that from the published MINER $\nu \mathrm{A}$ one-track $\nu_{\mu} \mathrm{QE}$ cross section, which does not reconstruct the proton track and calculates the $\mathrm{Q}_{\mathrm{QE} \text {,muon }}^{2} \mathrm{using}$ only the muon reconstructed observables (see Section 6.1). This comparison is extremely useful, 
because the recoil systems are handled differently for isolating the QE candidates and the background subtraction procedure varies for each analysis. Figures 6.1 and 6.2 show that the results are consistent for these independent QE analyses. Foremost, this also provides confidence in how the proton track is reconstructed and selected in the QE-like sample. Since the published MINER $\nu$ A one-track QE cross sections prefer a model that includes additional strength in the QE cross section due to meson exchange currents that result in the knockout of multinucleons, this implies that the $\nu_{\mu}$ QE-like data should also be sensitive to the dynamics in the nuclear medium.

Recall that the initial goal of the analysis was to measure the QE-like cross section and to characterize the proton transportation through the nucleus. However, the results from the model comparisons show that the QE-like cross section is extremely sensitive to the modeling of the pion production and transportation through the nucleus. GENIE predicts that the resonance production contributes more to the QE-like cross section than does NuWro. Based on the calculated $\chi^{2}$, GENIE better describes the $n$-track QE-like $\mathrm{d} \sigma / \mathrm{dQ}_{\mathrm{QE}-\text { like,proton }}^{2}$ extracted in this dissertation. However, the GENIE prediction does not include the contribution from meson exchange currents, which are necessary to describe the MINER $\nu \mathrm{A}$ one-track QE data, which perfers the NuWro RFG including the TEM.

The QE comparison results (see Section 6.1) and the disagreement between the

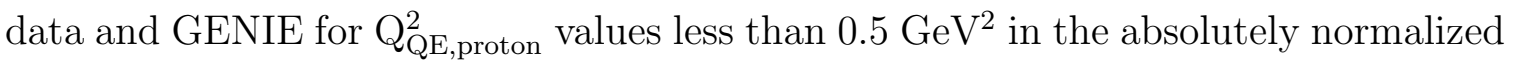
distribution confirm that the QE-like analysis is relatively sensitive to the $2 p-2 h$ meson exchange currents. There exist discrepancies between the NuWro predictions that account for these multinucleon contributions, as indicated in Figure 6.9, where the

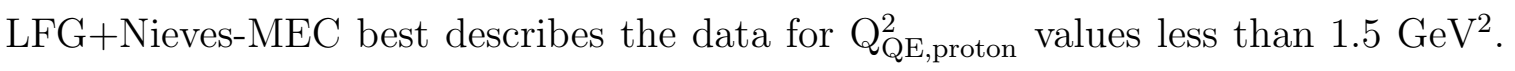
In addition, the Nieves-MEC contribution compensates for the weaker strength in the NuWro inelastic component of the cross section. Therefore, it becomes difficult to clearly interpret which component of the QE-like cross section is inaccurately mod- 
eled, the multinucleon contribution or the inelastic contribution based on the $n$-track QE-like data alone. Recall that the data from the one-track QE cross section is sensitive to multinucleon contributions from meson exchange currents and the inclusive pion analysis is more sensitive to the modeling of the pion production and transportation in the nucleus. Thus, the results from the QE-like cross section combined with the results from both the one-track QE and inclusive pion analyses will be the first step towards characterizing which components of the QE-like cross section are inaccurately modeled.

Since this analysis is sensitive to the modeling of the pion production in the region of $0.5<\mathrm{Q}_{\mathrm{QE} \text {,proton }}^{2}<1.5 \mathrm{GeV}^{2}$, it is a challenge to characterize the modeling of the proton propagation through the nucleus. However, for values of $\mathrm{Q}_{\mathrm{QE} \text {,proton greater }}^{2}$ than $1.5 \mathrm{GeV}^{2}$, all models failed to describe the data. As mentioned in Section 6.3, there are effective theoretical predictions that describe FSI effects fairly well in this region $\left(\mathrm{Q}_{\mathrm{QE} \text {,proton }}^{2}>1.5 \mathrm{GeV}^{2}\right)$. In order to fully understand and characterize the preformance of the INC models, it may become essential for neutrino event generators to incorporate these models.

This analysis shows that the QE-like cross section is useful for characterizing both the proton and pion propagation through the nucleus and the nuclear dynamics which result in the multinucleon contributions to the cross section due to meson exchange curents. The upstream region of the MINER $\nu \mathrm{A}$ detector is composed of pure Carbon, Iron, Lead passive materials. The near term goal is to measure the A-dependence of the effects of final state interactions utilizing the tools and analysis framework developed for this dissertation. 


\section{APPENDICES}




\section{APPENDIX A $\nu_{\mu}$ QE-LIKE KINEMATIC DISTRIBUTIONS}

This appendix show the resolutions, shape and absolutely normalized data/simulation comparisons, and ratios of the data to simulation for the $\nu_{\mu}$ QE-like candidates. The resolution distributions consists only of the true $\nu_{\mu}$ QE-like events that pass the selection criteria. In addition, these distributions are fitted with a double Gaussian. All distributions except for the muon kinematic variables are characterized based on the signal definition convoluted with GENIE, which is given by,

- CCQE-like $\equiv$ one negatively charge muon, no meson, no heavy baryons, no electromagnetic particles, and at least one proton in the final state

- Resonant $\equiv$ not CCQE-like and GENIE defined Resonant channel

- $\mathbf{D I S} \equiv$ not CCQE-like and GENIE define DIS channel

- Other $\equiv$ any interaction which does satisfies the any of above conditions.

The muon kinematic variables are classified based the reconstructed muon topology. Only the statistical error bars are shown on the plots. 

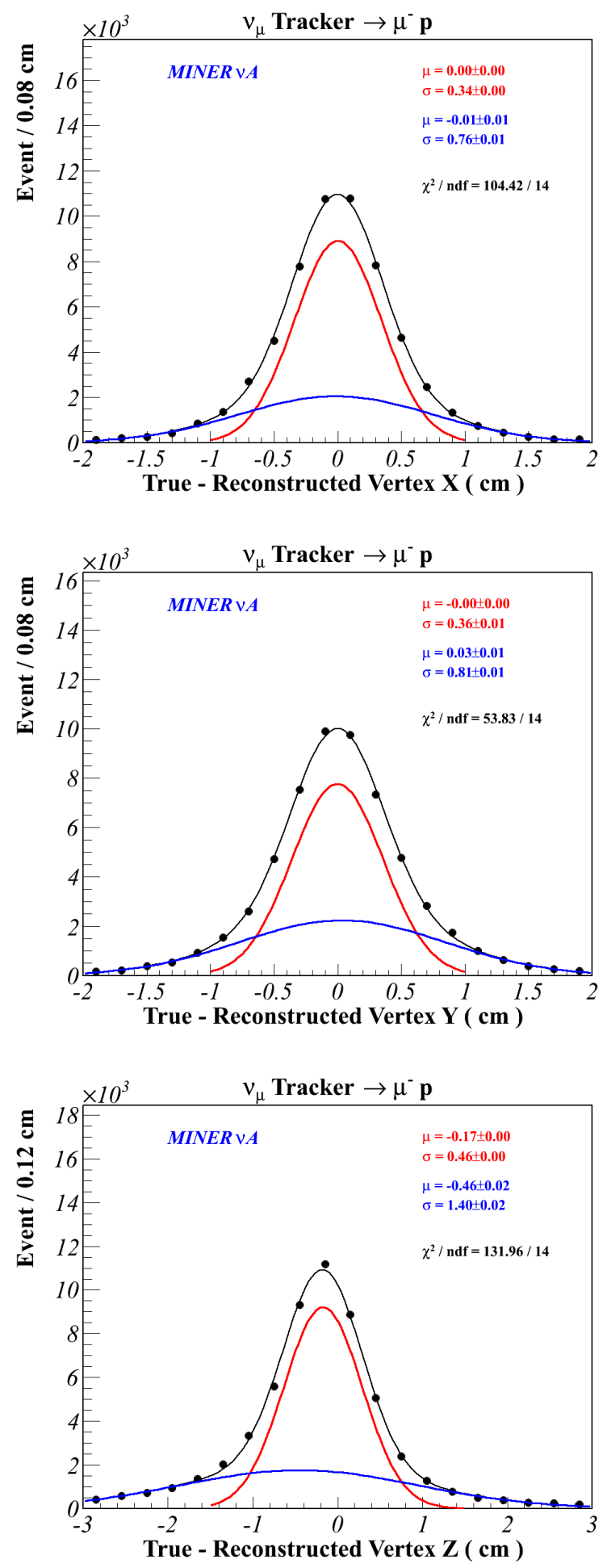

Figure A.1. Plotted are the reconstructed vertex X, Y, and Z residuals. 

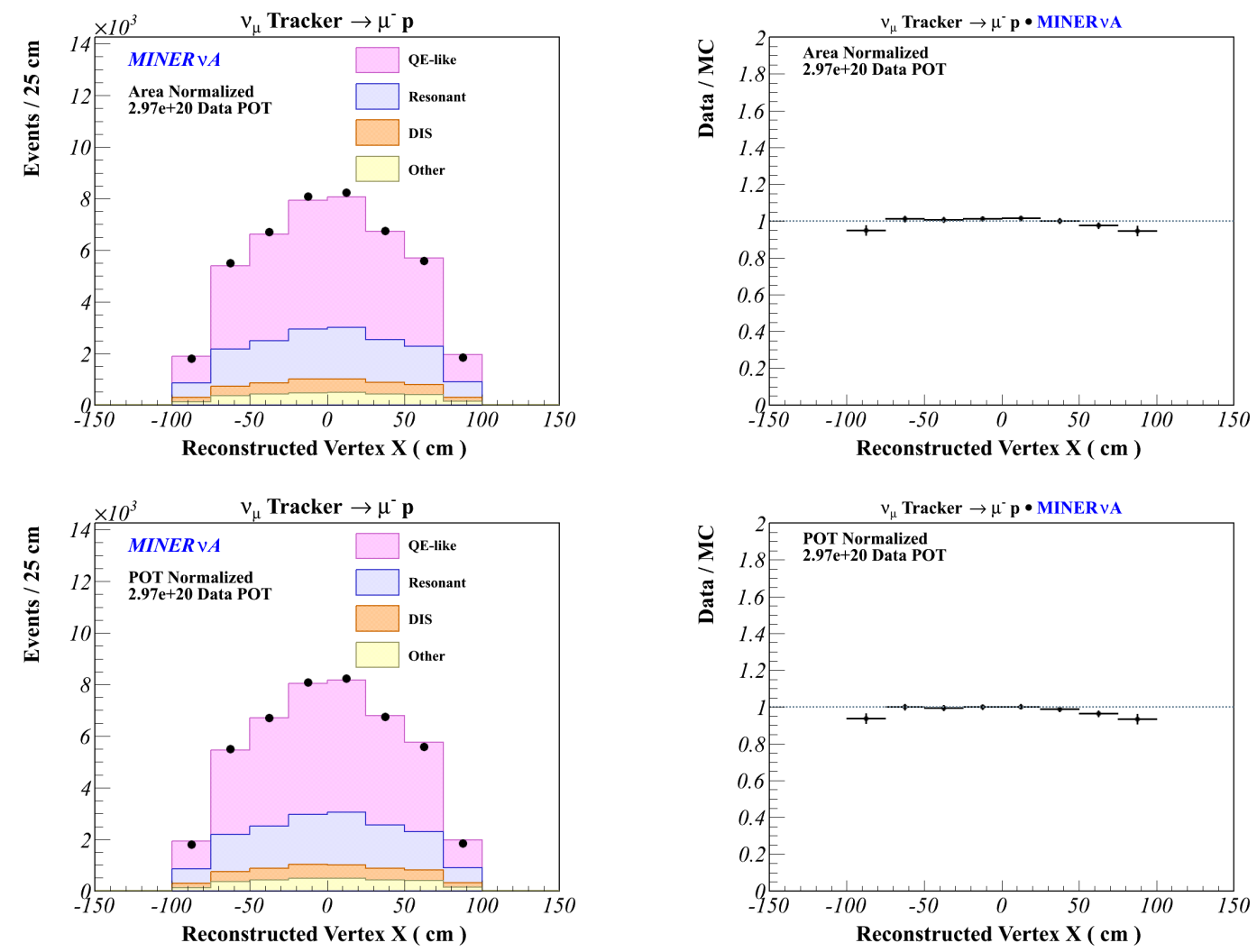

Figure A.2. Plotted are the area (top) and absolutely (bottom) normalized reconstructed vertex $\mathrm{X}$ position. 

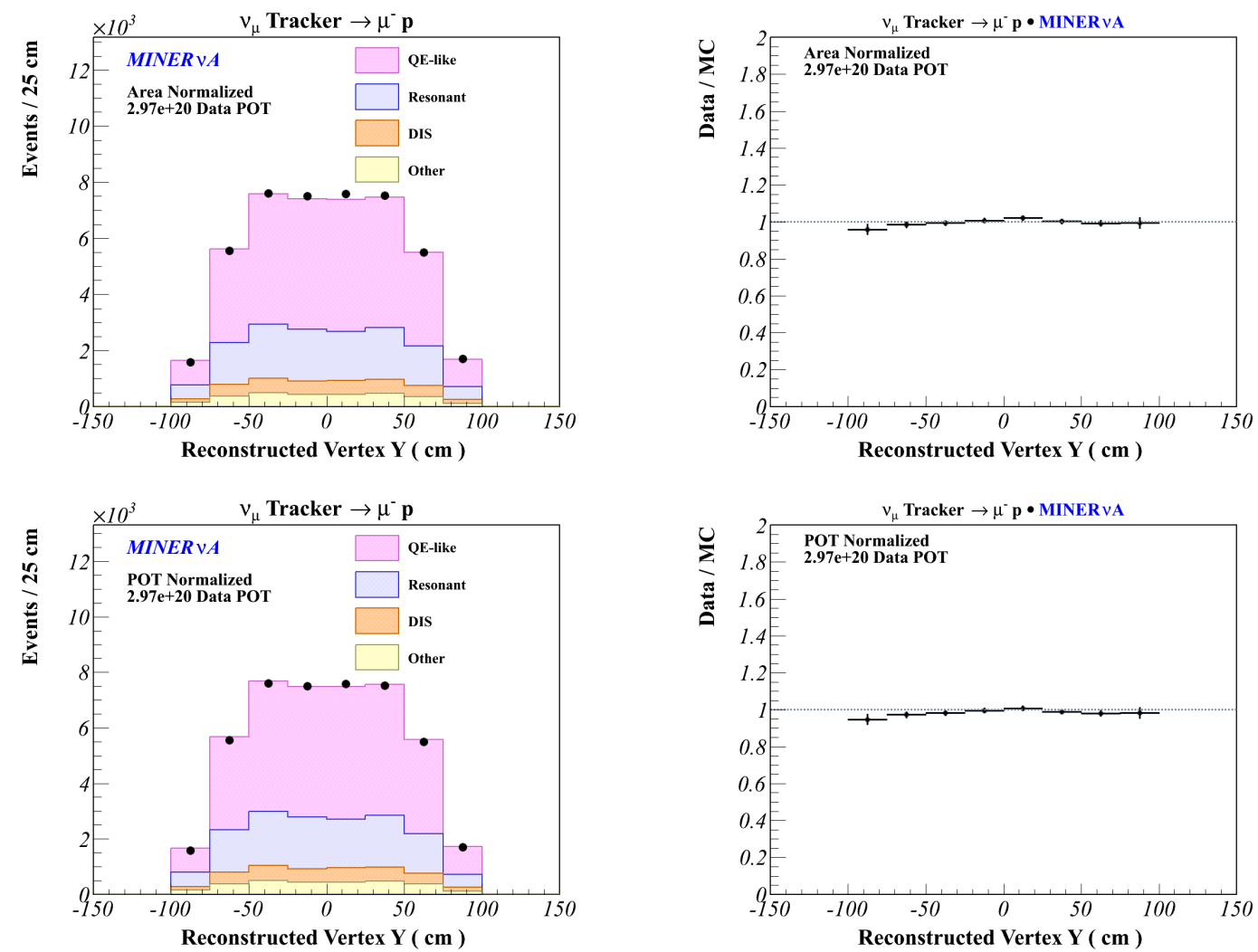

Figure A.3. Plotted are the area (top) and absolutely (bottom) normalized reconstructed vertex Y position. 

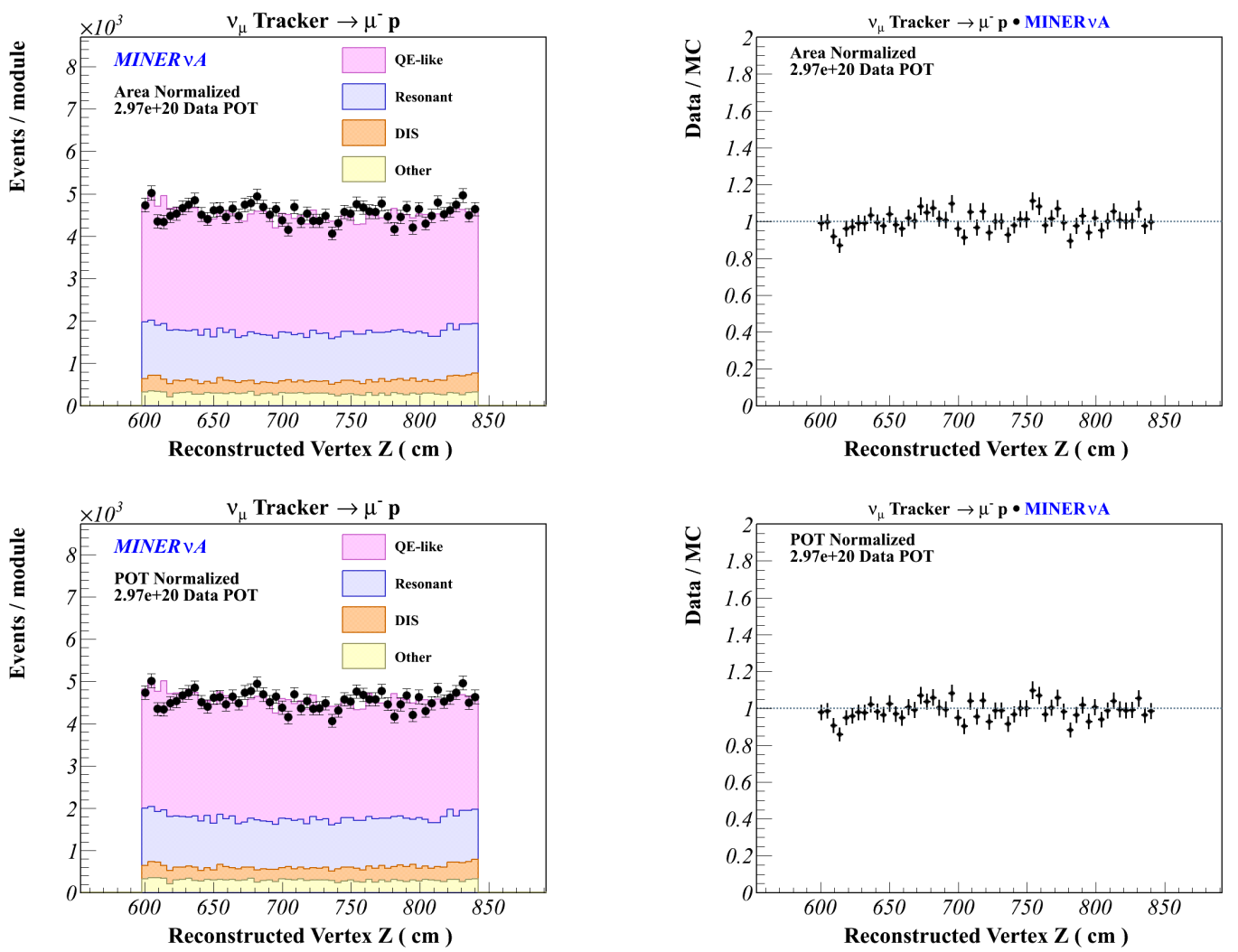

Figure A.4. Plotted are the area (top) and absolutely (bottom) normalized reconstructed vertex $\mathrm{Z}$ position. 

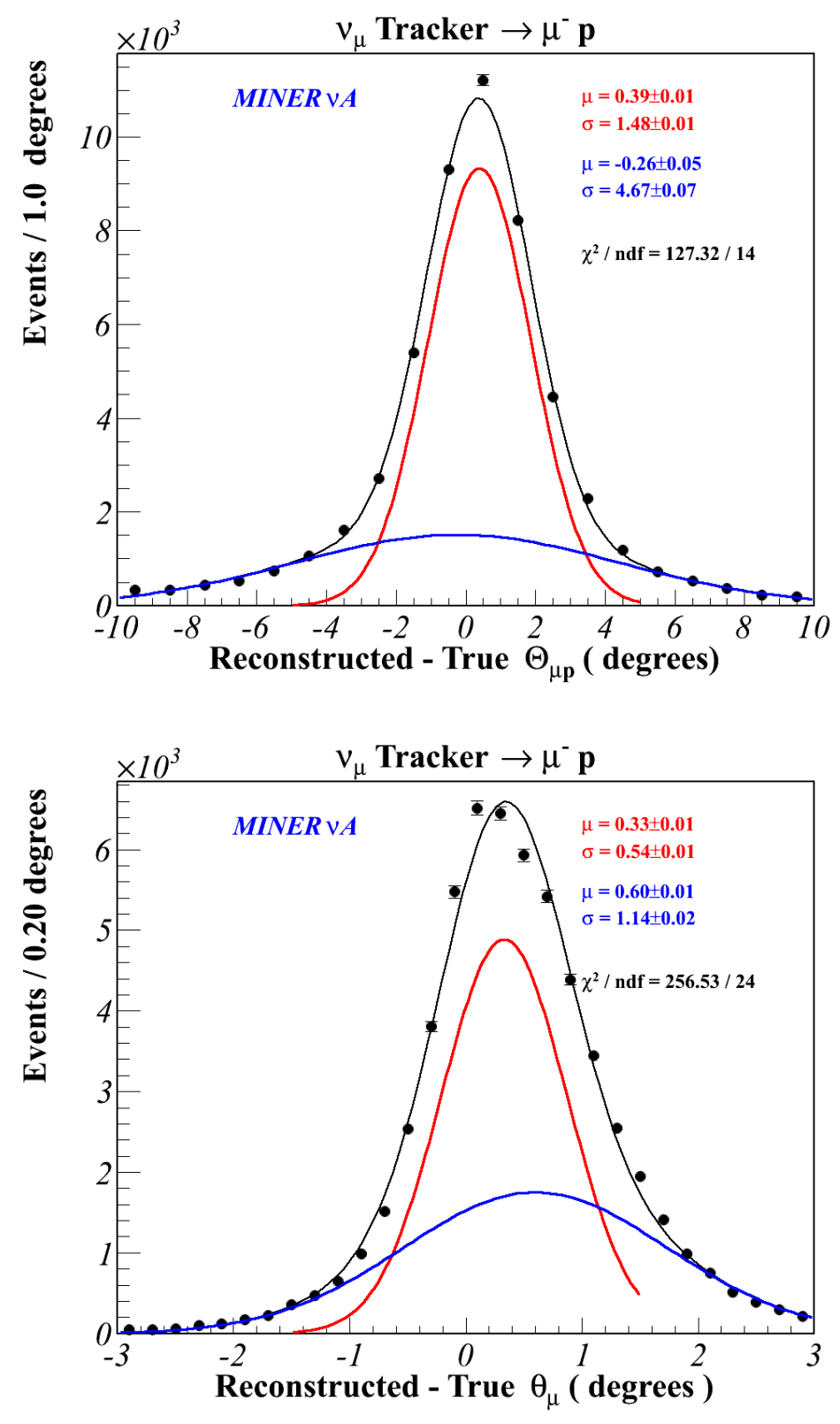

Figure A.5. Plotted are the reconstructed open angle and muon angle with respect to the beam axis residuals. 

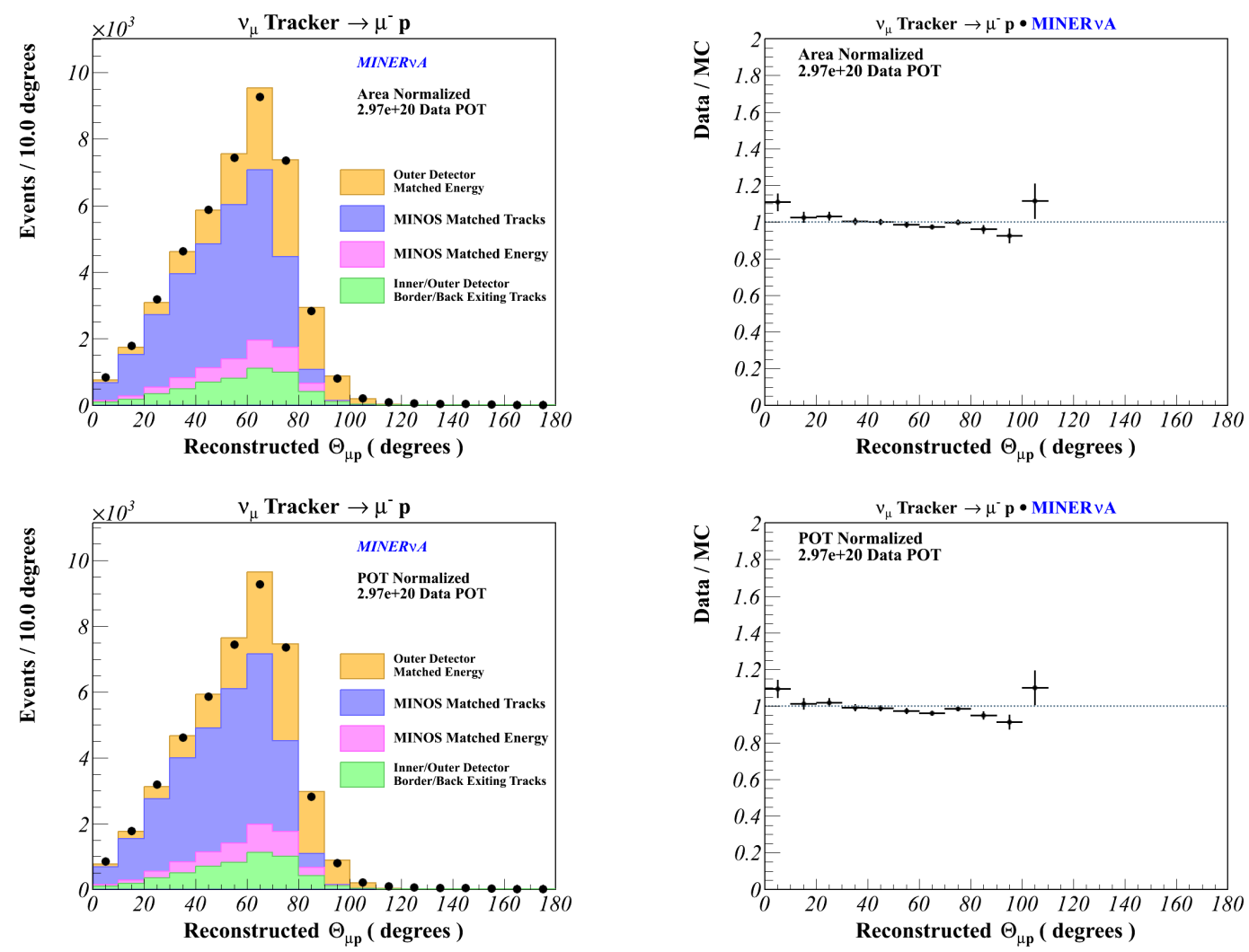

Figure A.6. Plotted are the area (top) and absolutely (bottom) normalized reconstructed open angle between the muon and leading order proton distributions. 

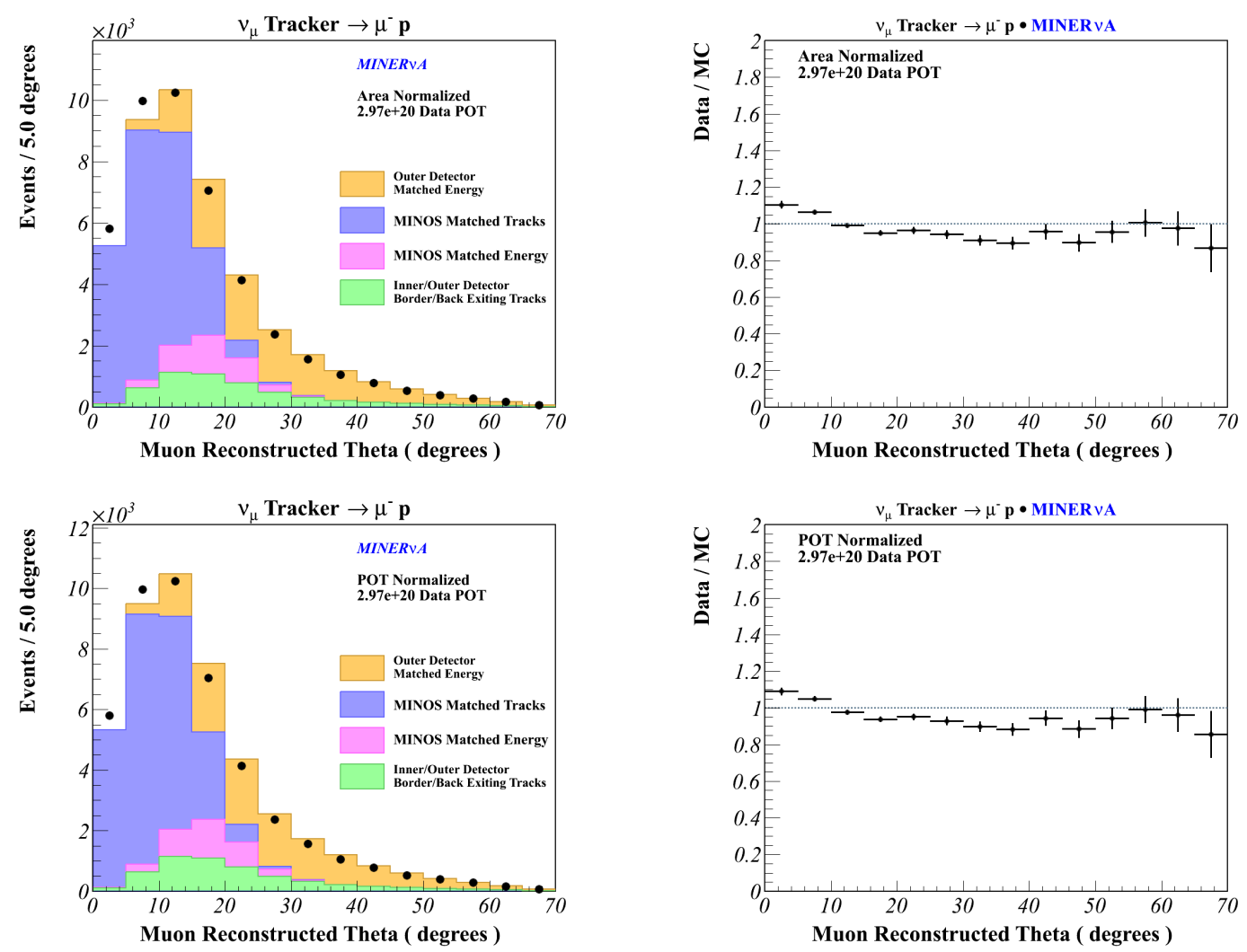

Figure A.7. Plotted are the area (top) and absolutely (bottom) normalized reconstructed muon's polar angle with respect to the beam distributions. 

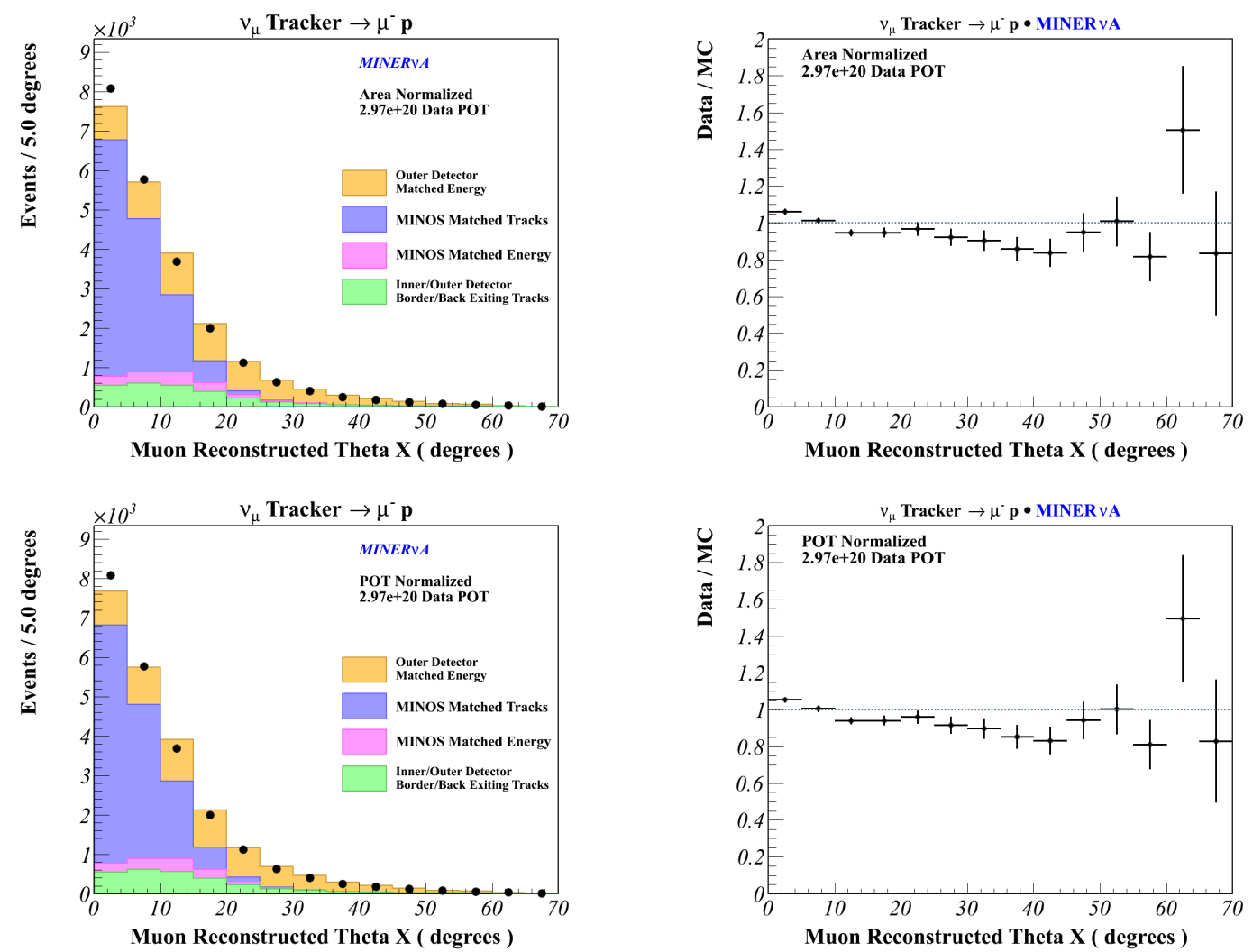

Figure A.8. Plotted are the area (top) and absolutely (bottom) normalized reconstructed muon's polar angle projected onto the x-axis with respect to the beam distributions. 

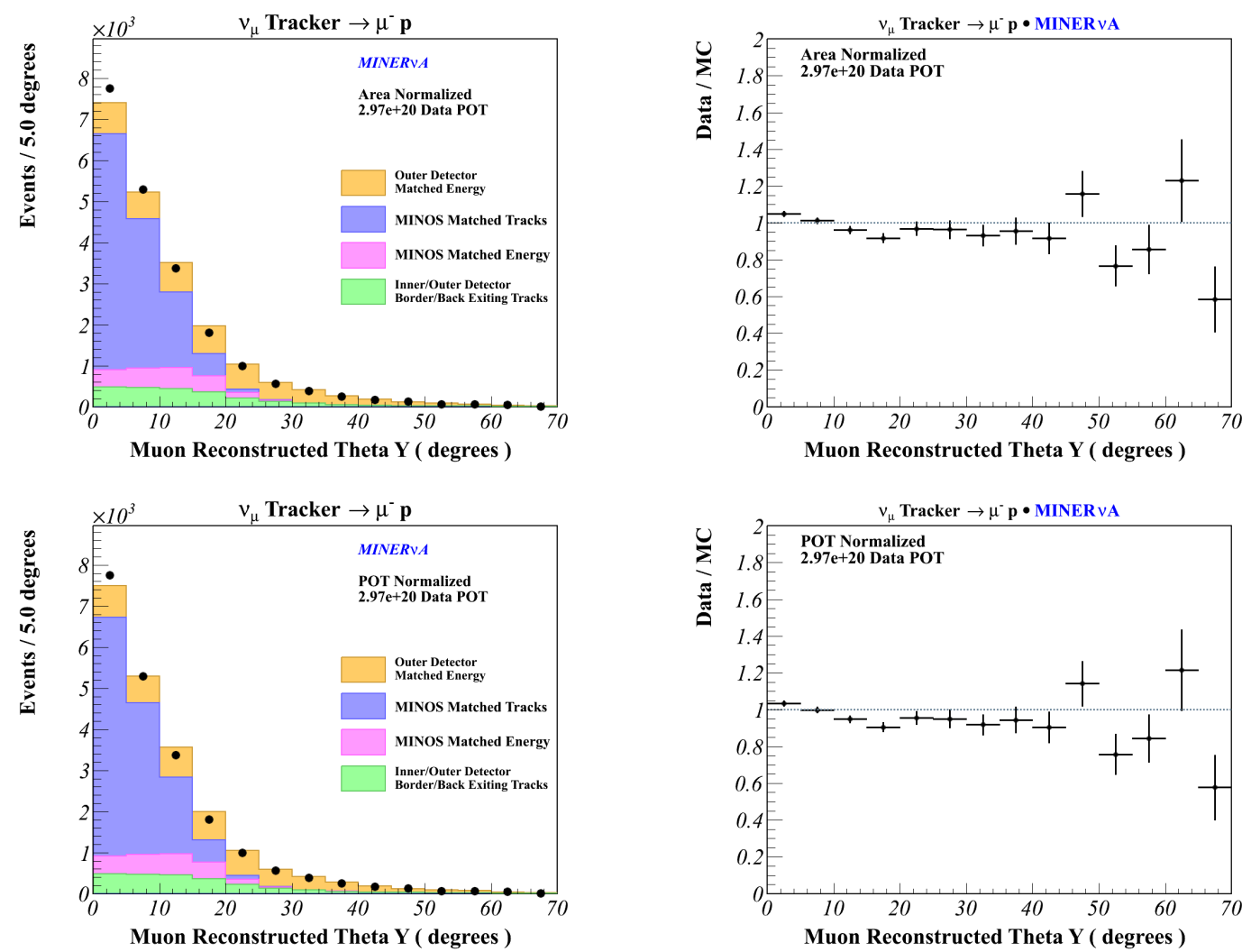

Figure A.9. Plotted are the area (top) and absolutely (bottom) normalized reconstructed muon's polar angle projected onto the $y$-axis with respect to the beam distributions. 

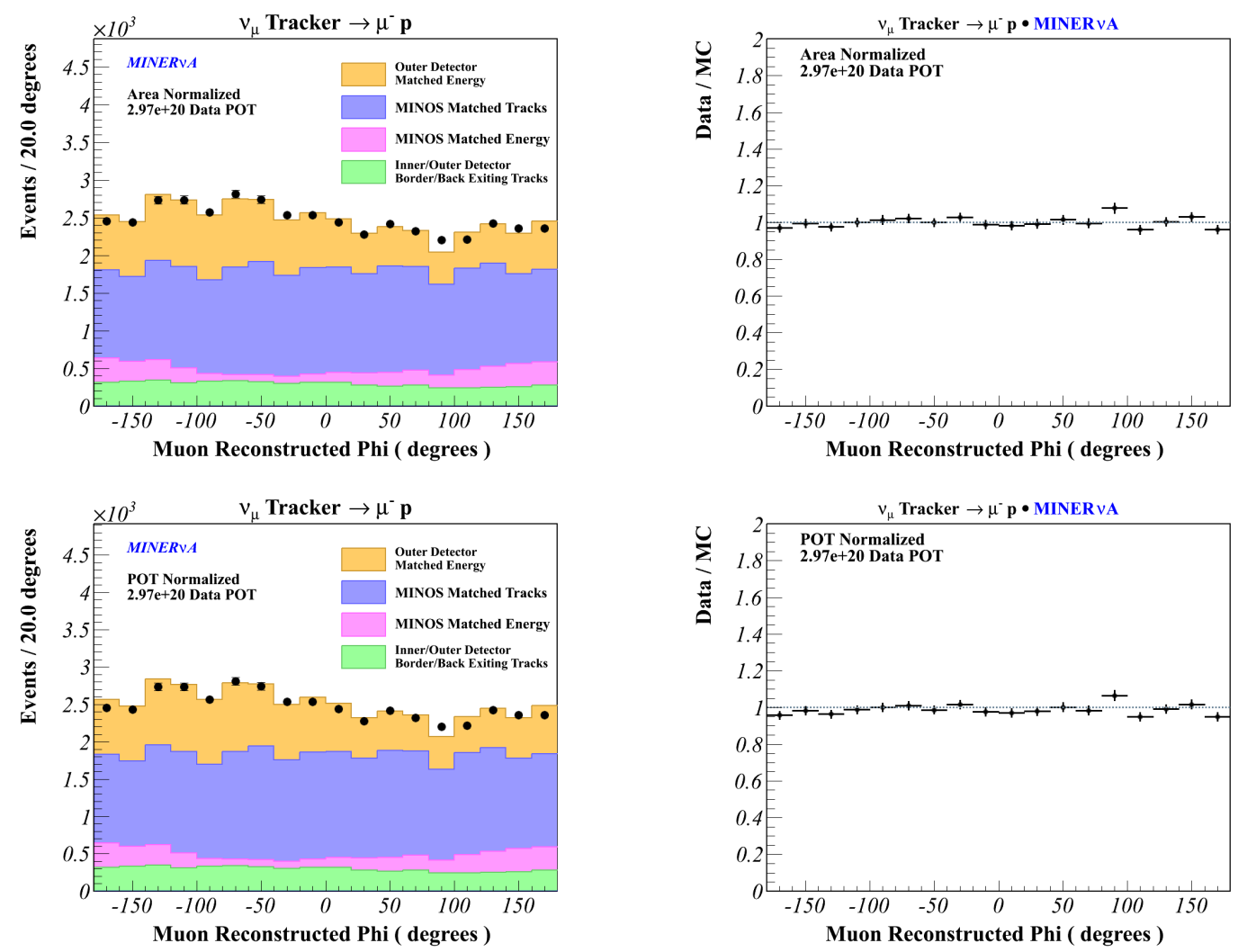

Figure A.10. Plotted are the area (top) and absolutely (bottom) normalized reconstructed muon's azimuthal angle with respect to the beam distributions. 

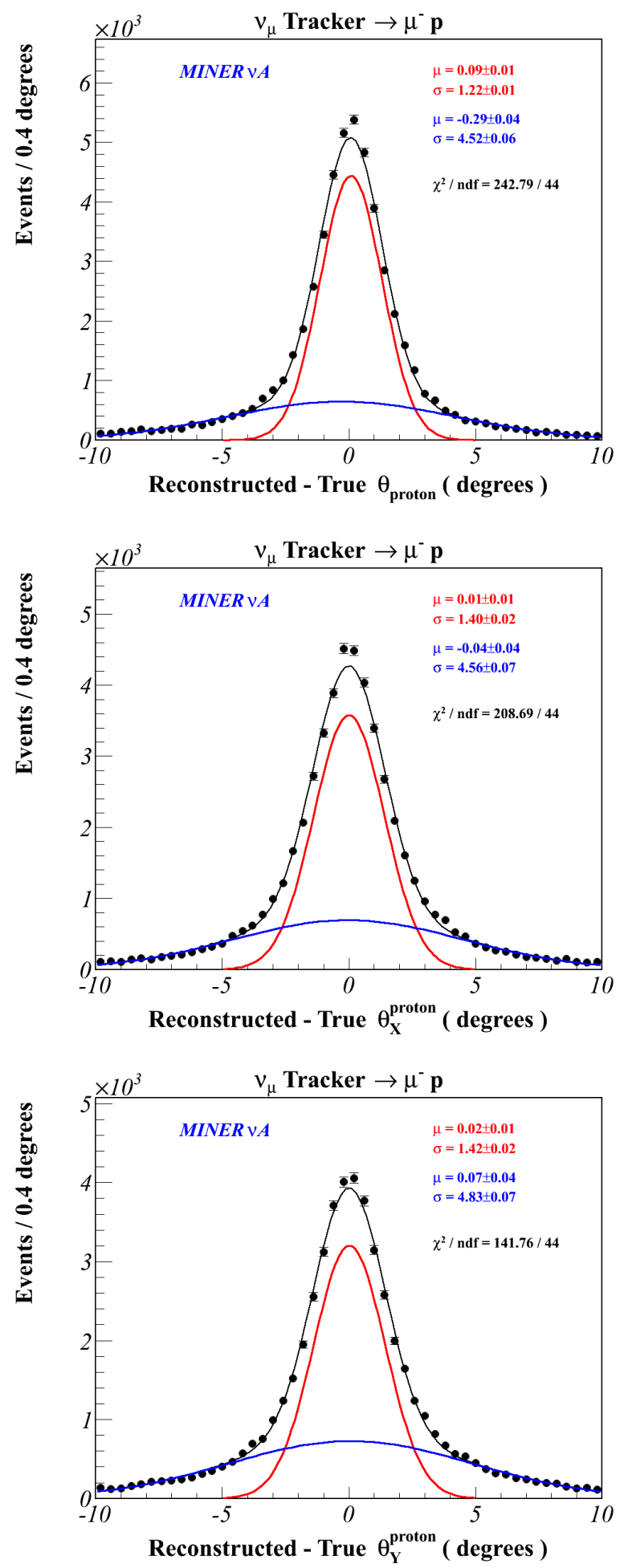

Figure A.11. Plotted are the reconstructed leading proton angles with respect to the beam axis residuals. 

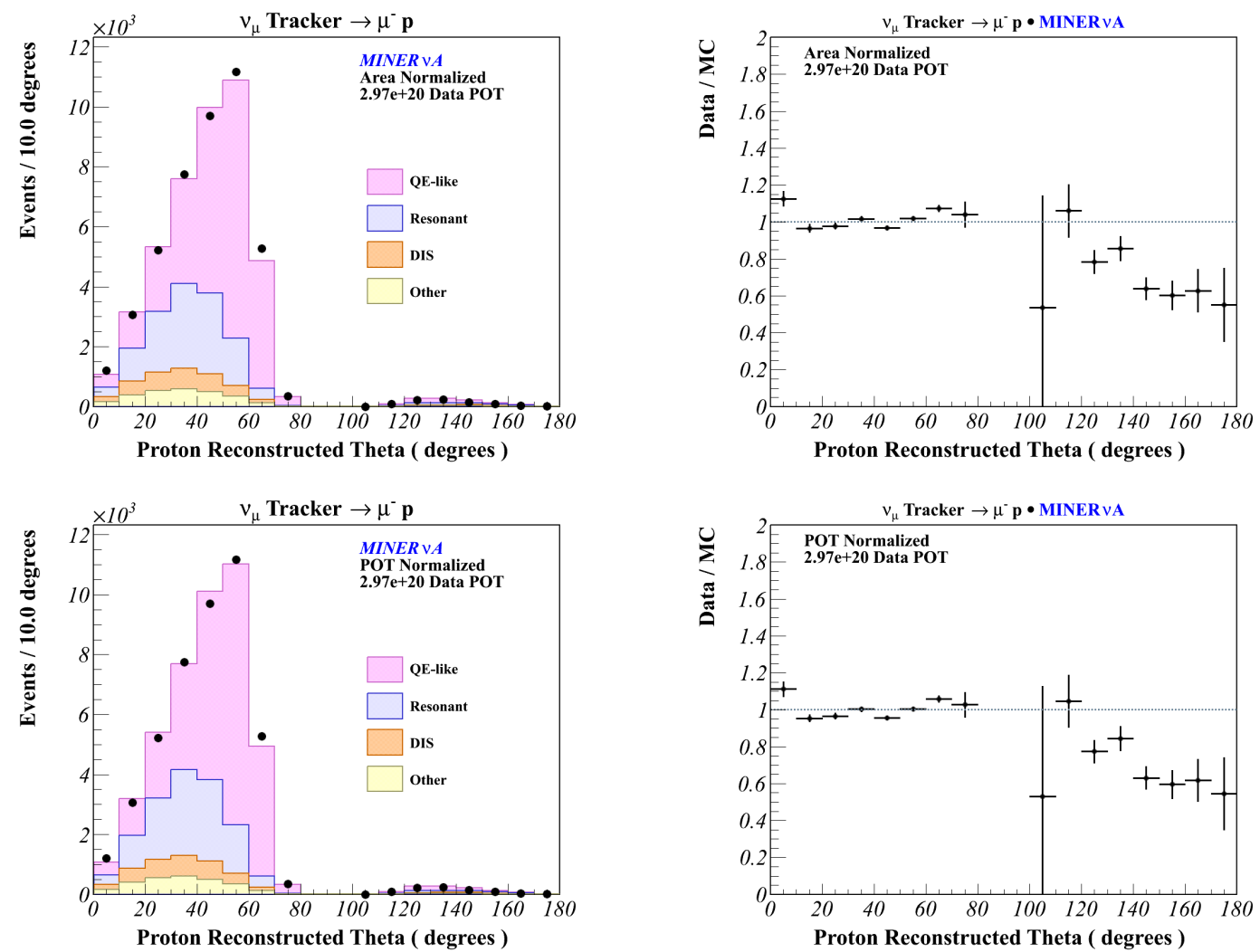

Figure A.12. Plotted are the area (top) and absolutely (bottom) normalized reconstructed leading proton's polar angle with respect to the beam axis distributions. 

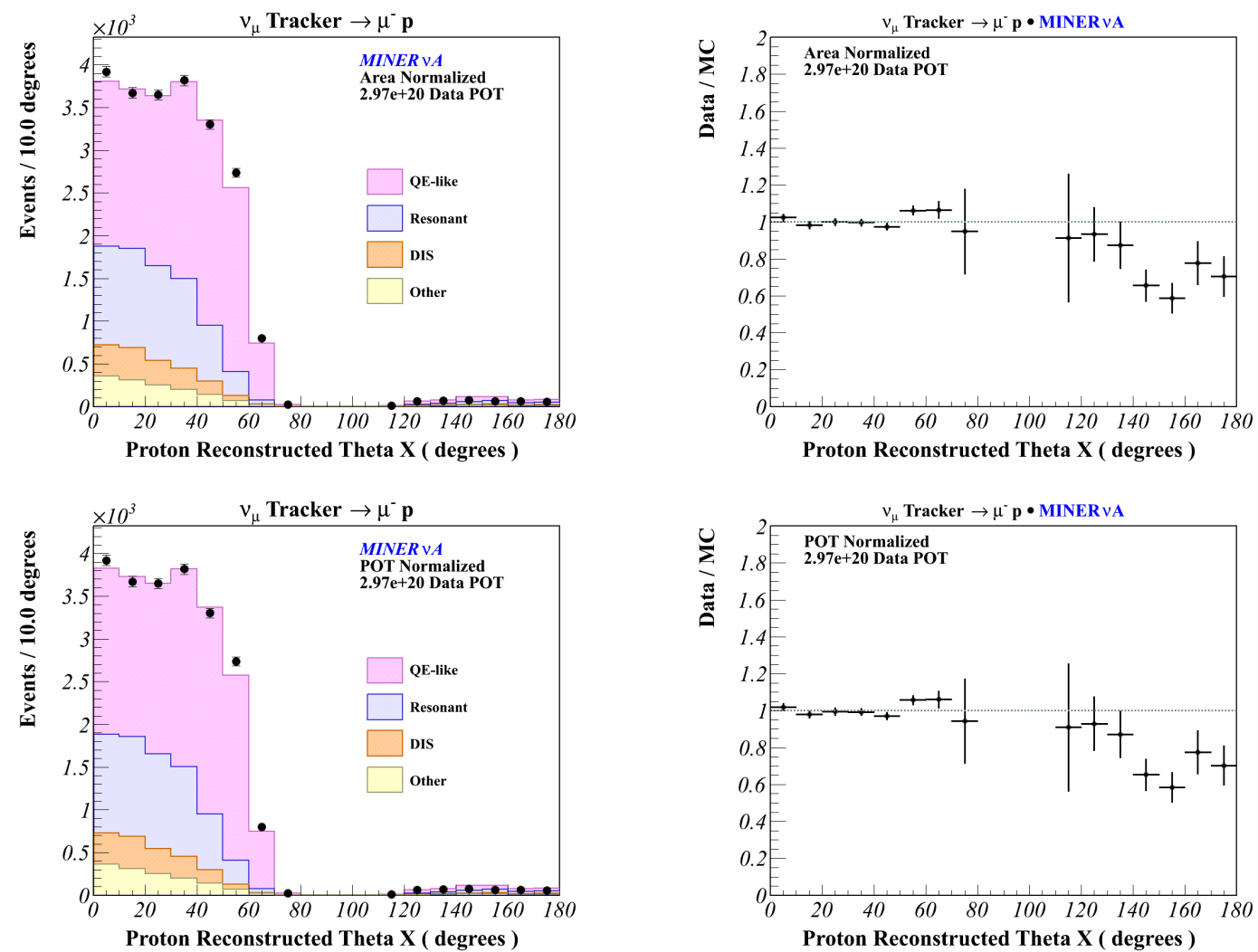

Figure A.13. Plotted are the area (top) and absolutely (bottom) normalized reconstructed leading proton's polar angle projected onto the x-axis with respect to the beam axis distributions. 

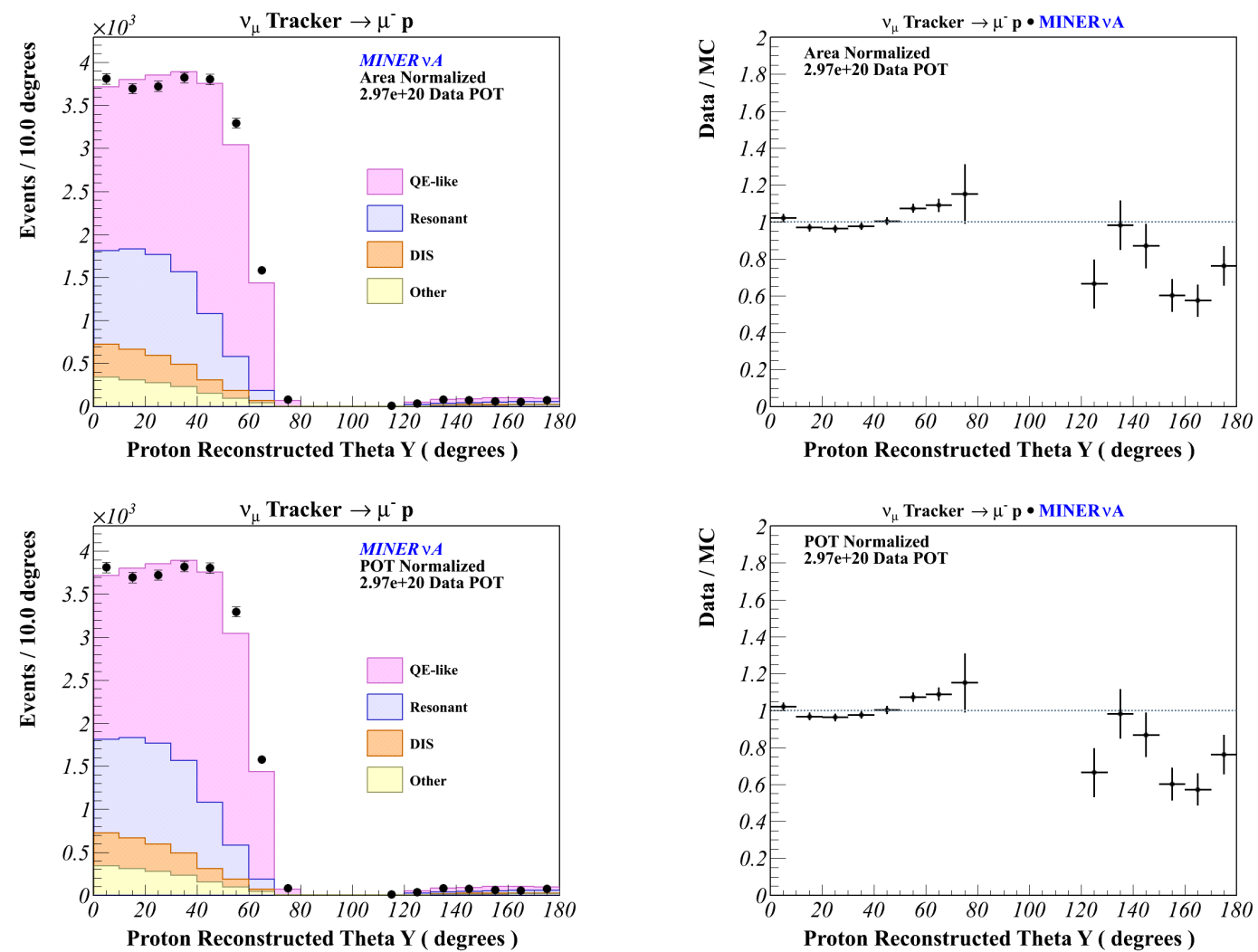

Figure A.14. Plotted are the area (top) and absolutely (bottom) normalized reconstructed leading proton's polar angle projected onto the y-axis with respect to the beam axis distributions. 

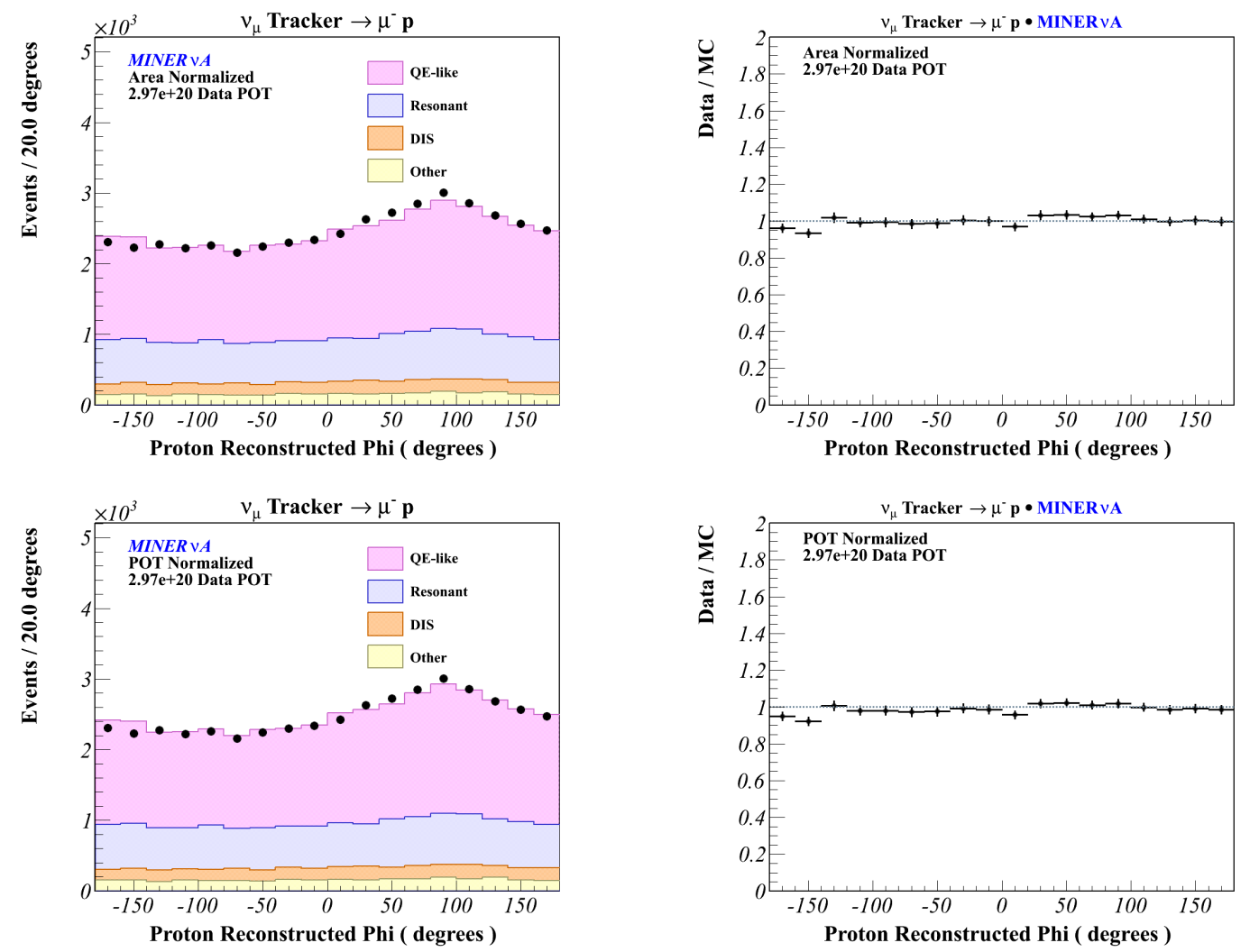

Figure A.15. Plotted are the area (top) and absolutely (bottom) normalized reconstructed leading proton's azimuthal angle with respect to the beam axis distributions. 

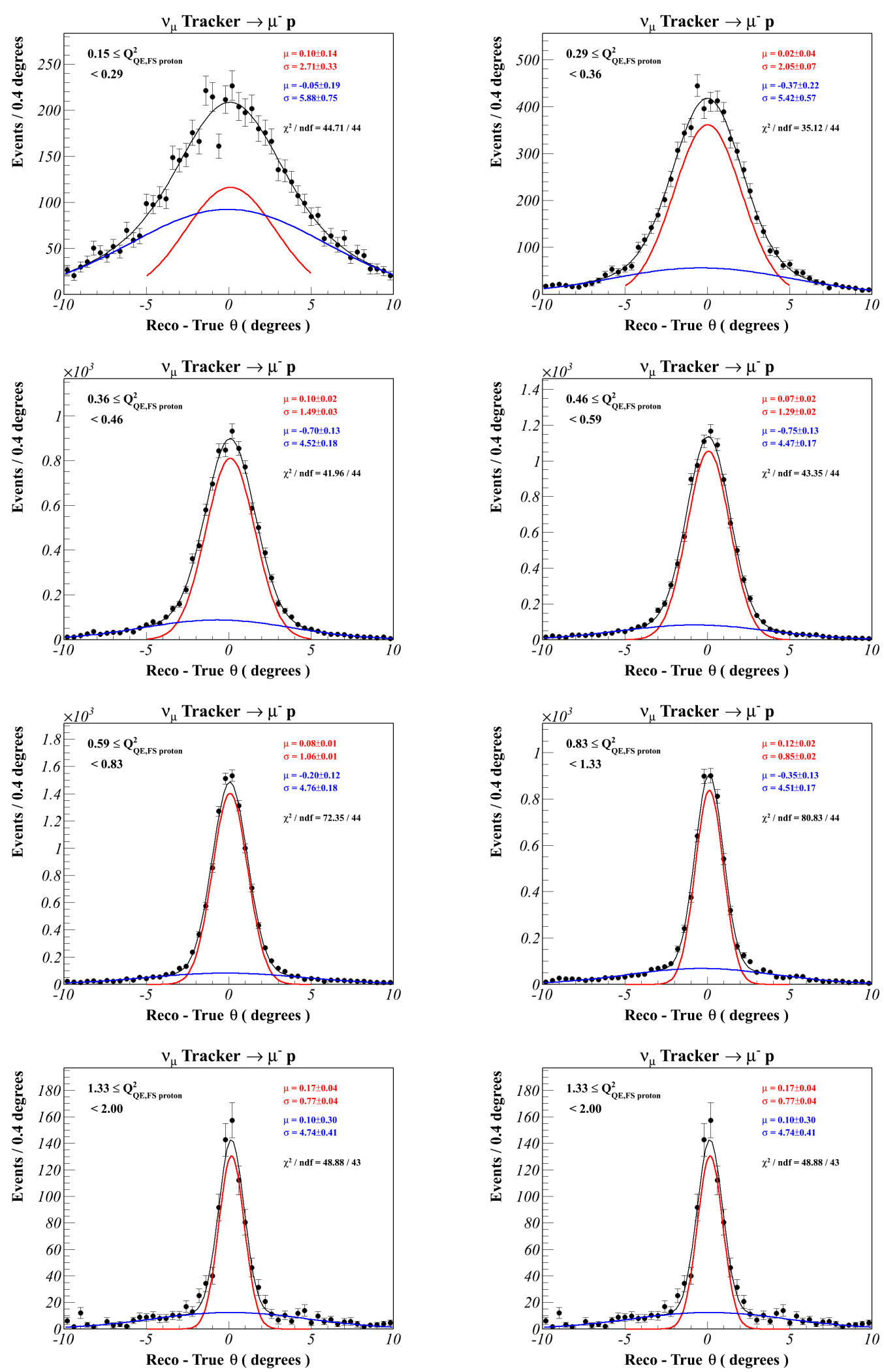

Figure A.16. Plotted are the reconstructed leading proton angle with respect to the beam axis residual in the measured $\mathrm{Q}_{\mathrm{QE} \text {,proton }}^{2}$ bins. 

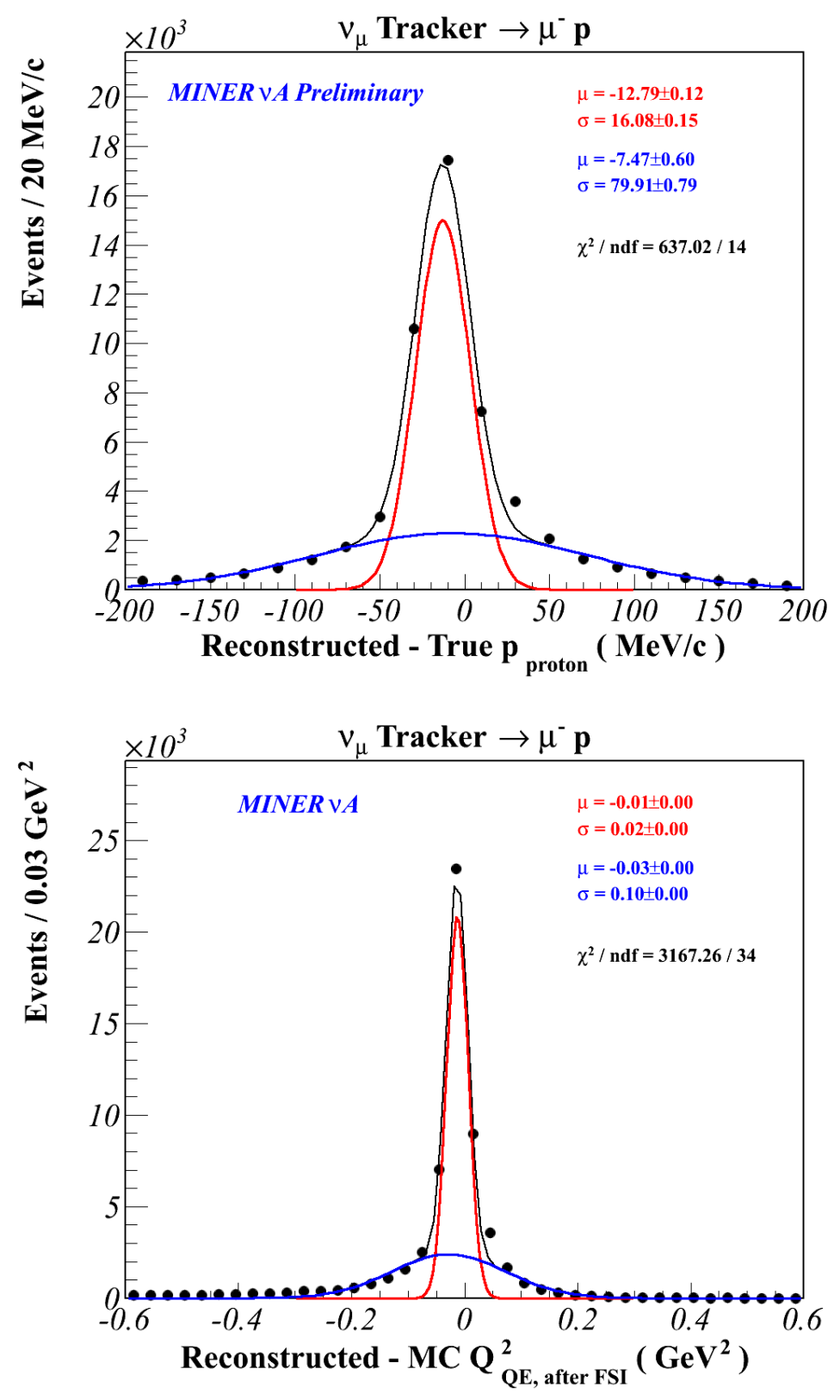

Figure A.17. Plotted are the reconstructed leading proton momentum (left) and $\mathrm{Q}_{\mathrm{QE} \text {,proton }}^{2}$ (right) residuals. 

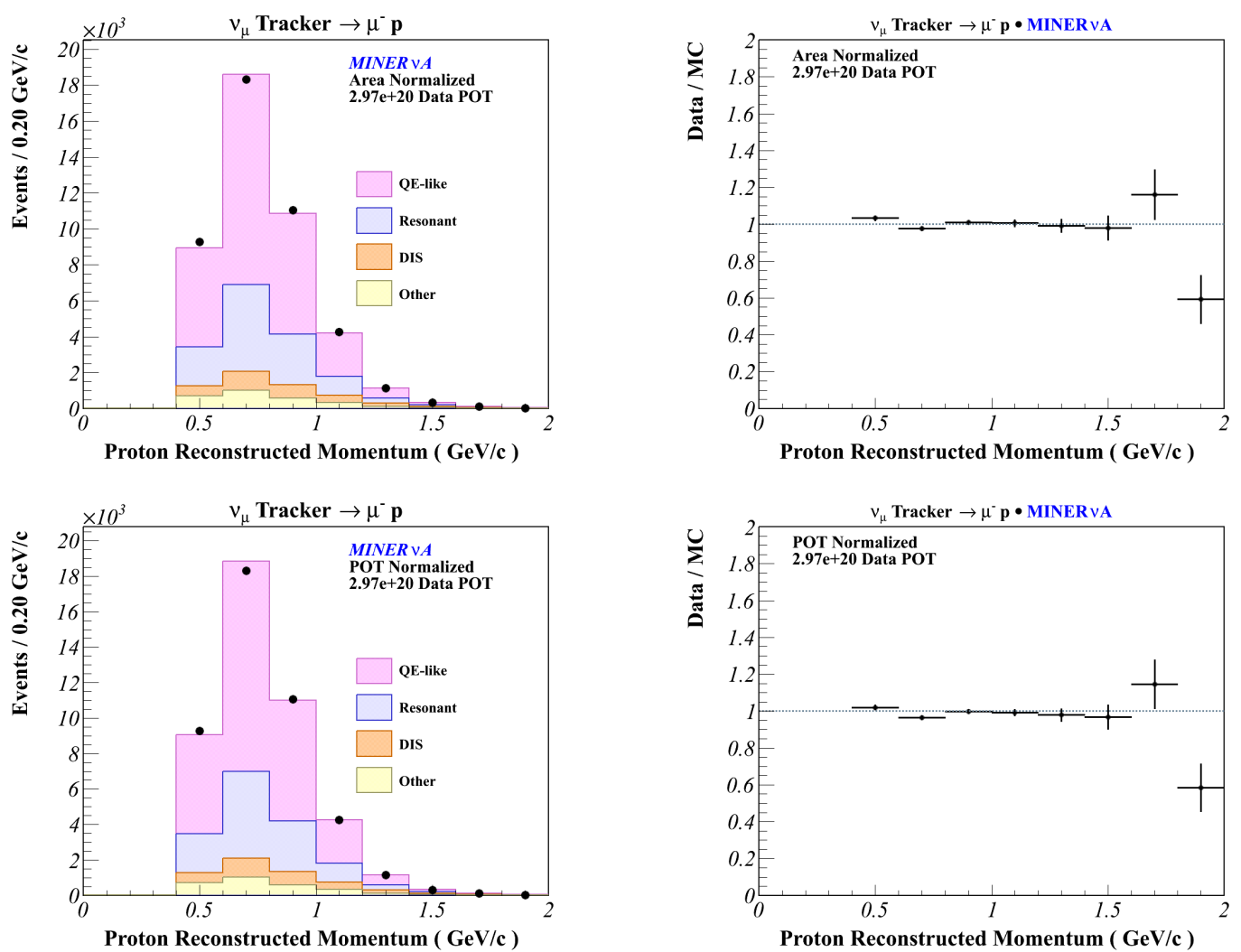

Figure A.18. Plotted are the area (top) and absolutely (bottom) normalized reconstructed leading proton momentum. 

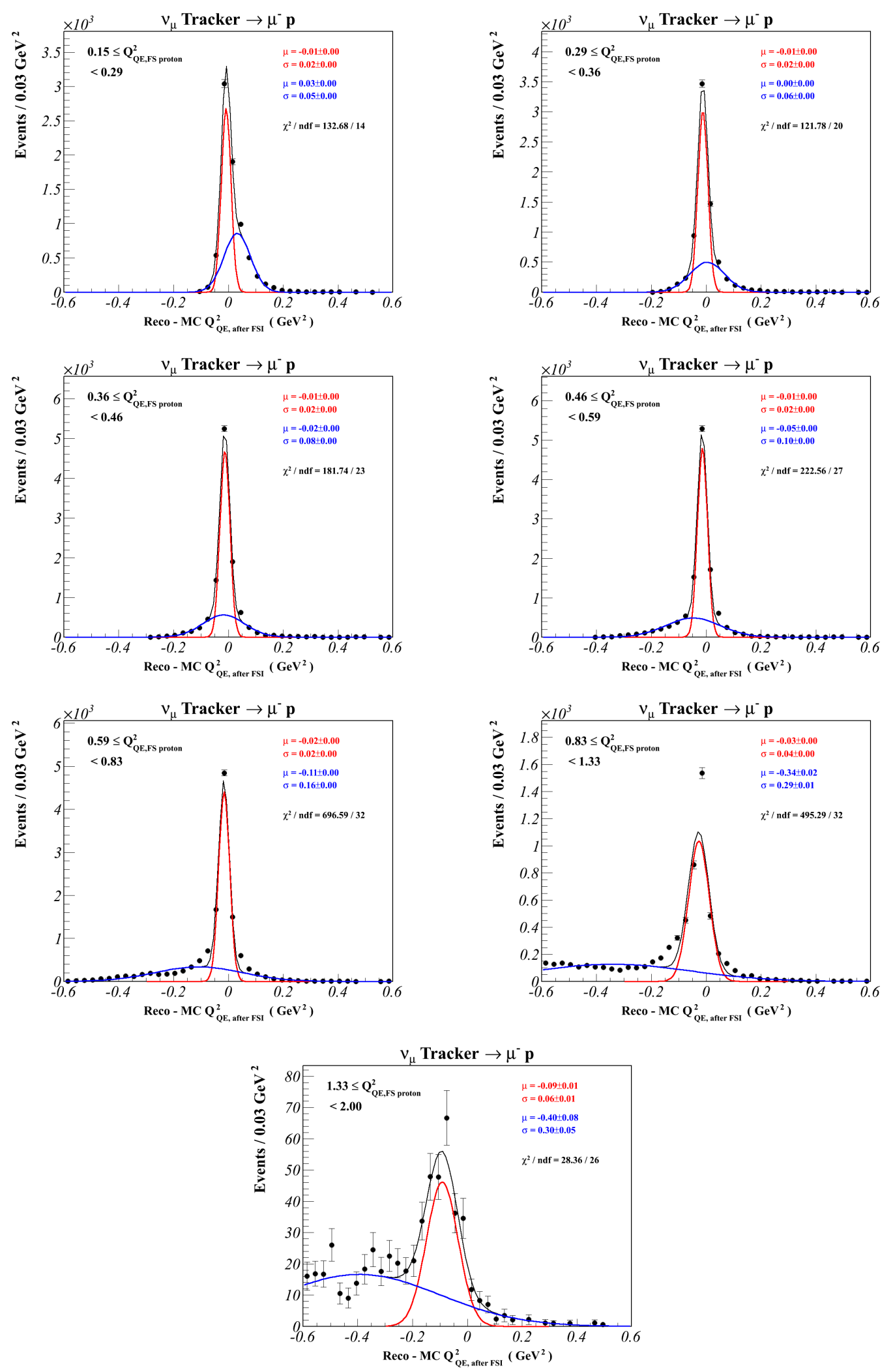

Figure A.19. Plotted are the reconstructed $\mathrm{Q}_{\mathrm{QE} \text {,proton }}^{2}$ residual in the measured $\mathrm{Q}_{\mathrm{QE} \text {,proton }}^{2}$ bins. 


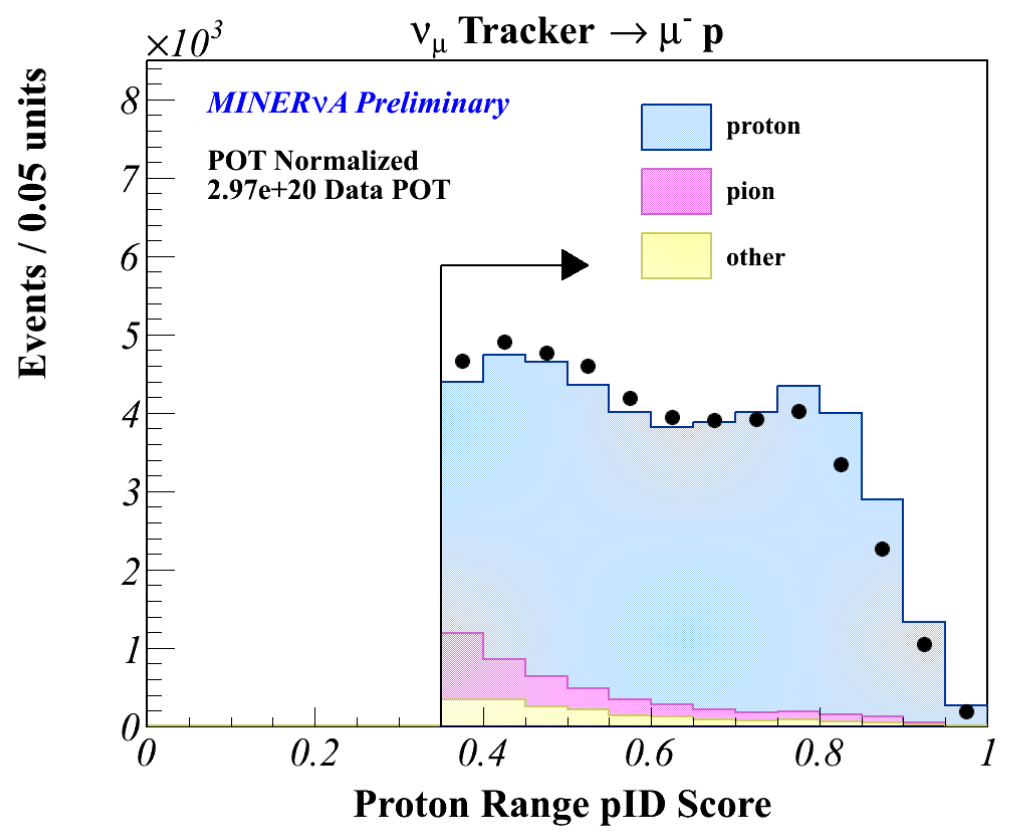

Figure A.20. Plotted is the proton pID score after applying all of the analysis cuts which isolated the $\nu_{\mu}$ QE-like candidates. 


\section{APPENDIX B \\ MUON-PROTON $\nu_{\mu}$ QE-LIKE KINEMATIC DISTRIBUTIONS}

Presented in this appendix are additional kinematic distributions for the MINOSmatched $\nu_{\mu}$ QE-like candidates. These plots are produced with assistance from M. Betancourt of the MINER $\nu \mathrm{A}$ collaboration.

1. The $\mathrm{Q}^{2}$ difference, $\mathrm{Q}_{\mathrm{QE} \text {,muon }}^{2}-\mathrm{Q}_{\mathrm{QE} \text {,proton }}^{2}$ is presented in Figure B.1.

2. Figure B.2 shows the proton transverse momentum, which is given by

$$
p_{\text {proton }}^{T}=p_{\mu} \sin \theta_{\mu} / \sin \theta_{\text {proton }}
$$

3. The neutron transverse momentum is demonstrated in Figure B.3 and defined as,

$$
p_{\text {neutron }}^{T}=\left(E_{\mu}+E_{\text {proton }}+E_{\text {bind }}-E_{\nu}\right)^{2}-p_{\text {neutron }}^{\|}-M_{\text {neutron }}^{2},
$$

where the longitudinal neutron momentum $\mathrm{p}_{\text {neutron }}^{\|}$is given as

$$
p_{\text {neutron }}^{\|}=p_{\mu} \cos \theta_{\mu}+p_{\text {proton }} \cos \theta_{\text {proton }}-E_{\nu} .
$$

4. Figure B.4 gives the difference, $\Delta \theta=\theta^{\text {pred }}-\theta^{\text {reco }}$, between the reconstructed angle of the leading proton track and the predicted angle for a two-body system, where $\theta_{\text {proton }}^{\text {pred }}$ is written as

$$
\theta_{\text {proton }}=\cos ^{-1}\left(\frac{E_{\nu}-p_{\mu} \cos \theta_{\mu}}{p_{\text {proton }}}\right)
$$



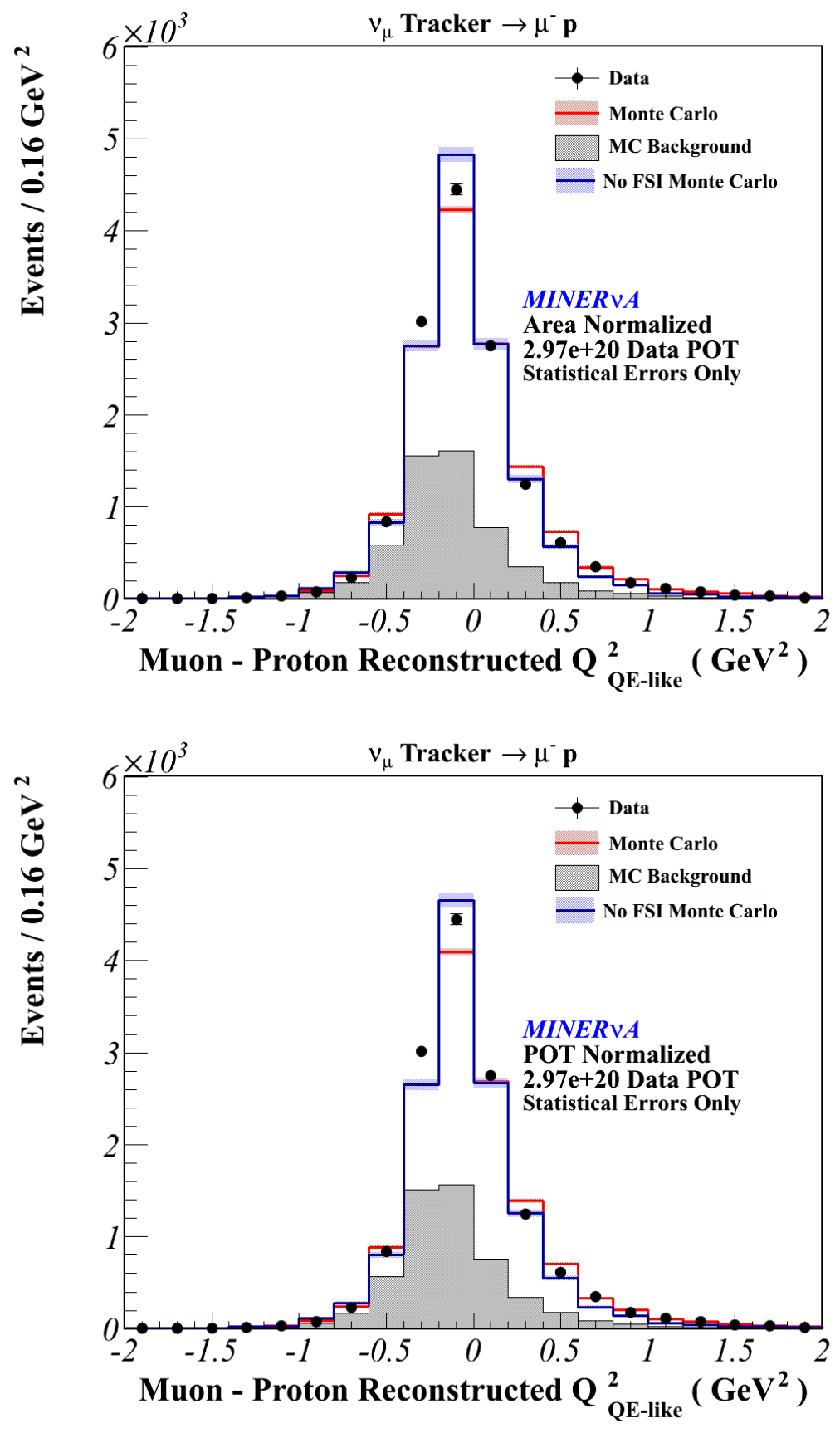

Figure B.1. The reconstructed $\mathrm{Q}^{2}$ difference between the muon and proton kinematics, area(top) and absolutely(bottom) normalized data and Monte Carlo comparisons. 

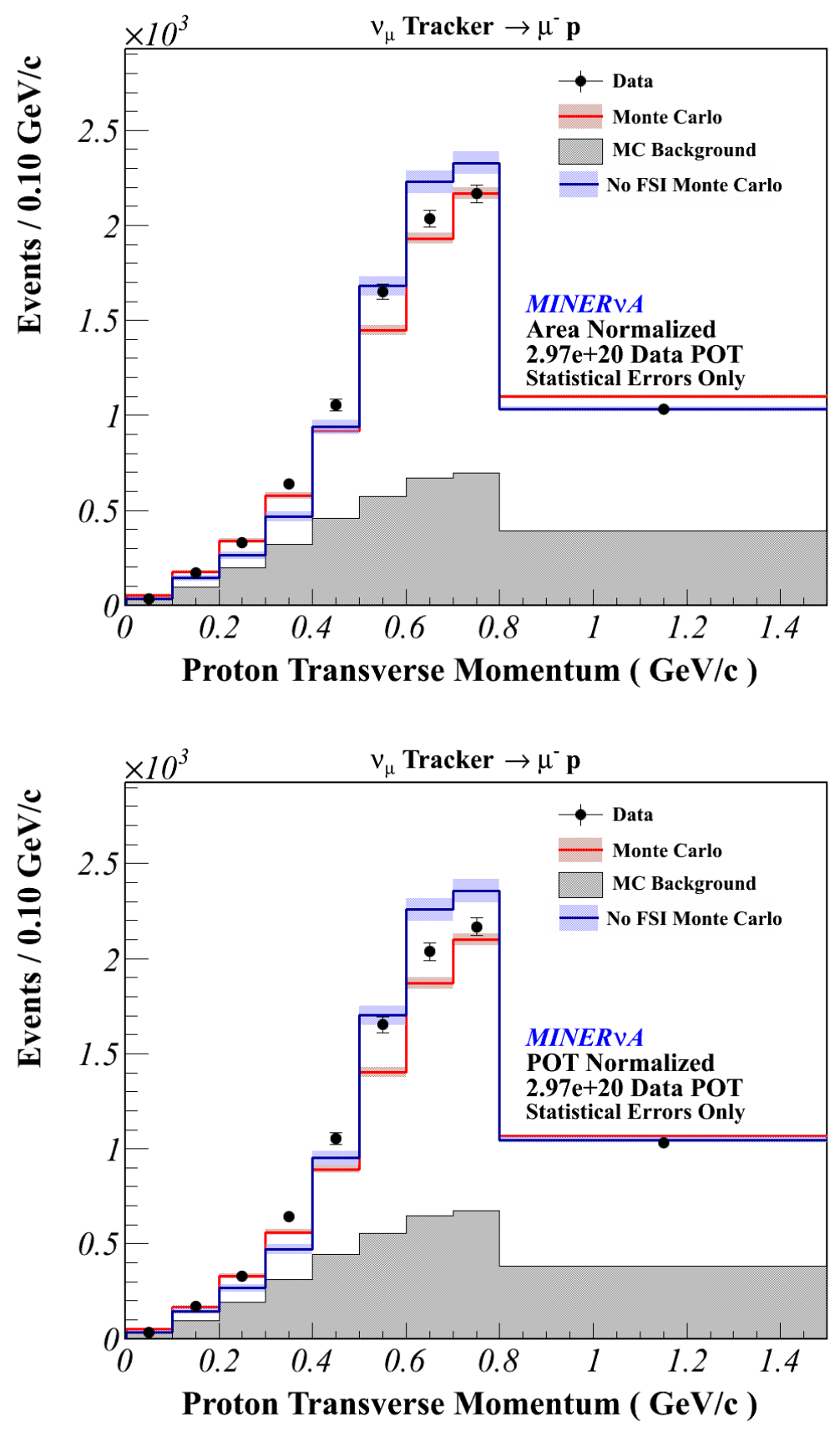

Figure B.2. The area(top) and absolutely(bottom) normalized data and Monte Carlo comparisons of the proton transverse momentum. 

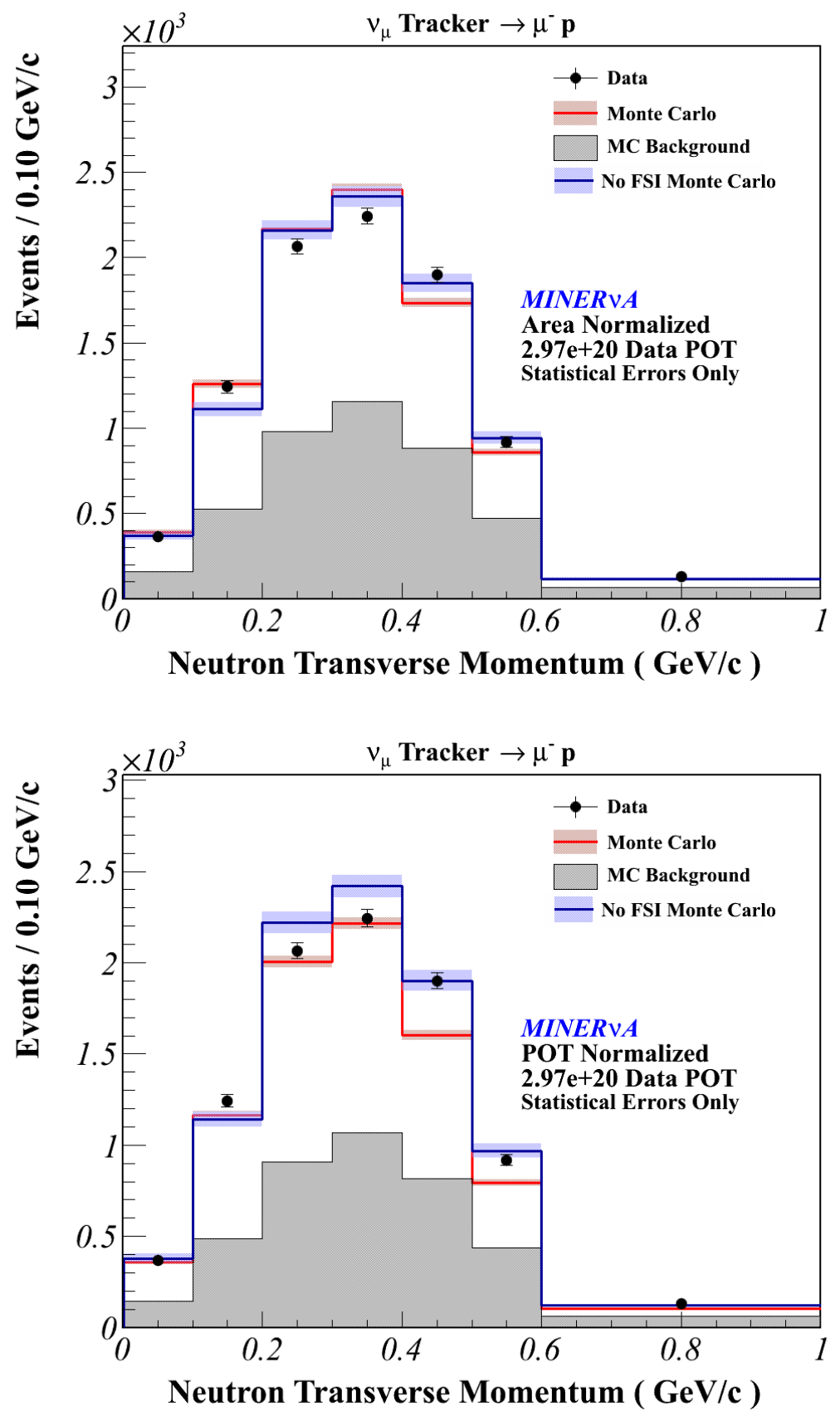

Figure B.3. The area(top) and absolutely(bottom) normalized data and Monte Carlo comparisons of the neutron transverse momentum. 

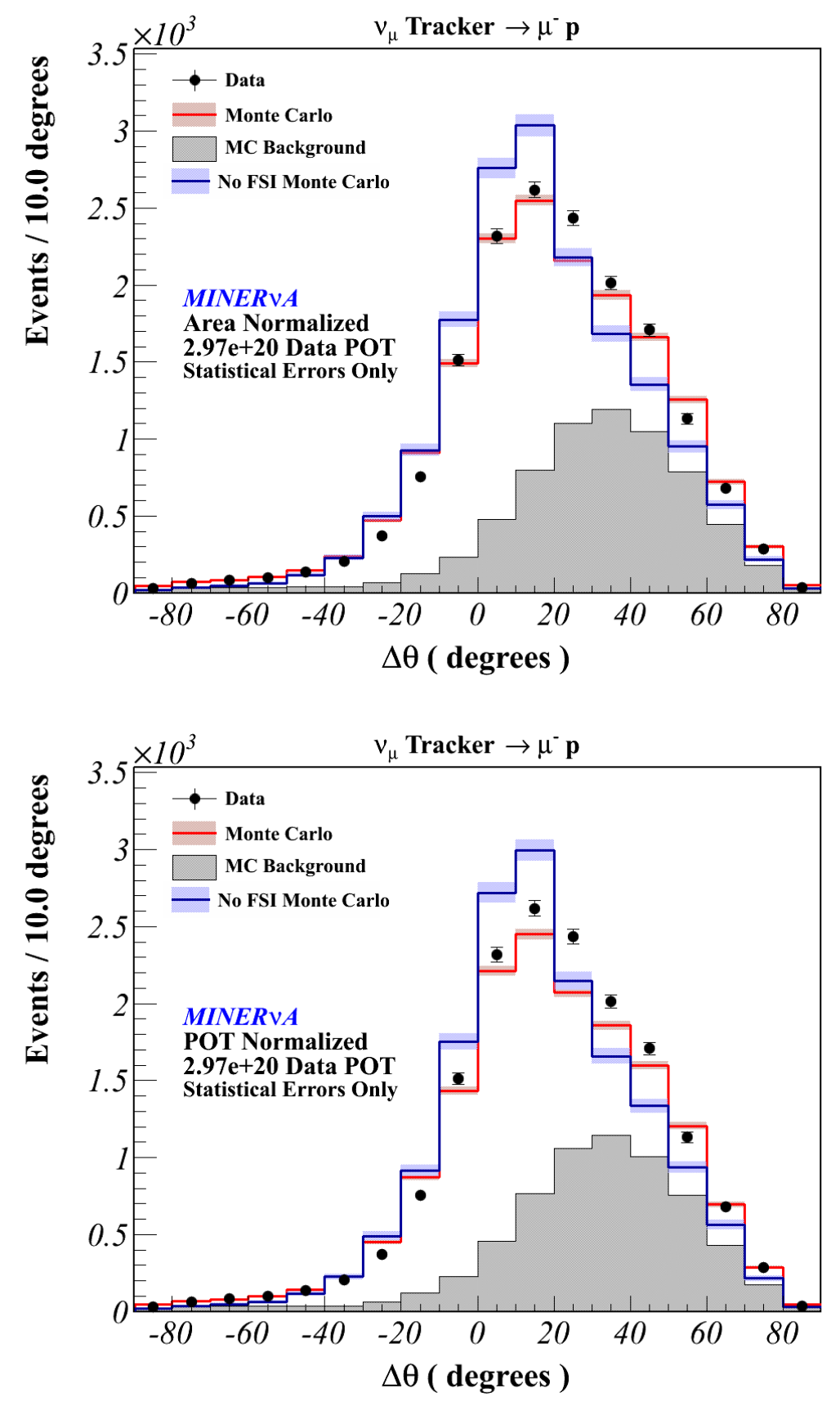

Figure B.4. The differences between the projected and reconstructed proton angle for the area(top) and absolutely(bottom) normalized data and Monte Carlo comparisons. 


\section{APPENDIX C \\ $\nu_{\mu}$ QE-LIKE EFFICIENCY DISTRIBUTIONS}

Presented in the appendix are plots that illustrated the product of the efficiency and acceptance and the purity for the $\nu_{\mu}$ QE-like signal, which is given by

$$
\epsilon_{i}=\left(\frac{N_{g e n}^{\text {pass }}}{N_{\text {gen }, \text { fid }}}\right)_{i},
$$

where $\mathrm{N}_{\text {gen,fid }}$ is the number of signal events generated in the fiducial volume for bin $i$ and $\mathrm{N}_{\text {gen }}^{\text {pass }}$ is the number of generated signal events that appear in the signal selection for bin $i$.
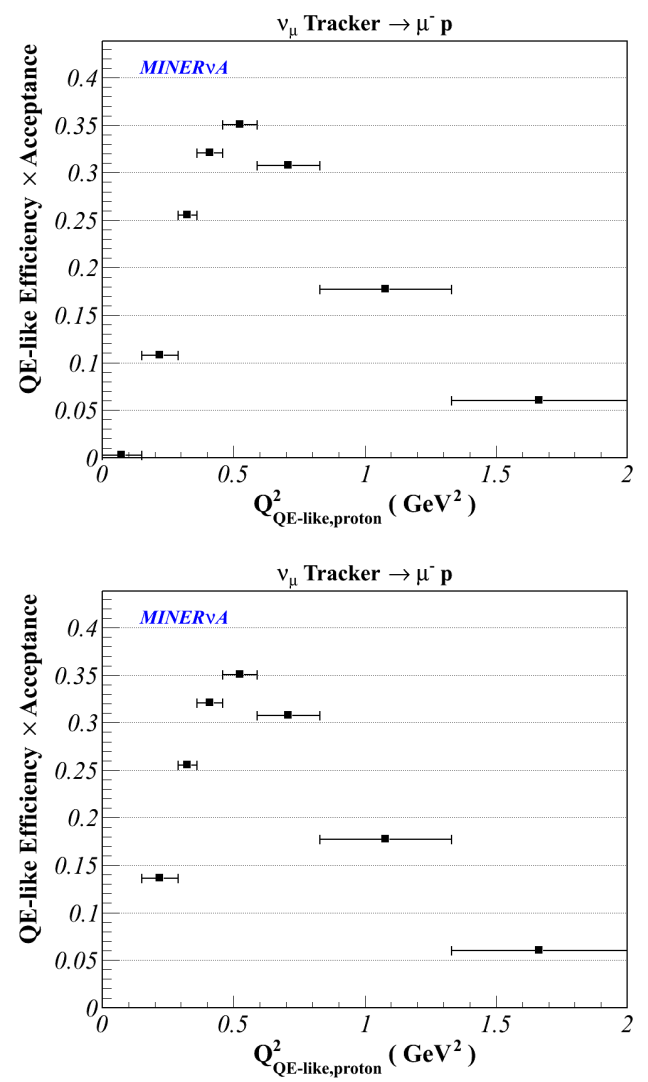

Figure C.1. Plotted is the efficiency as function of $\mathrm{Q}_{\mathrm{QE} \text {,proton }}^{2}$ for the $\nu_{\mu}$ QE-like signal without (top) and with (bottom) the condition on the proton's momentum (at least one proton in the final state with momentum greater than $450 \mathrm{MeV} / \mathrm{c}$ ). 

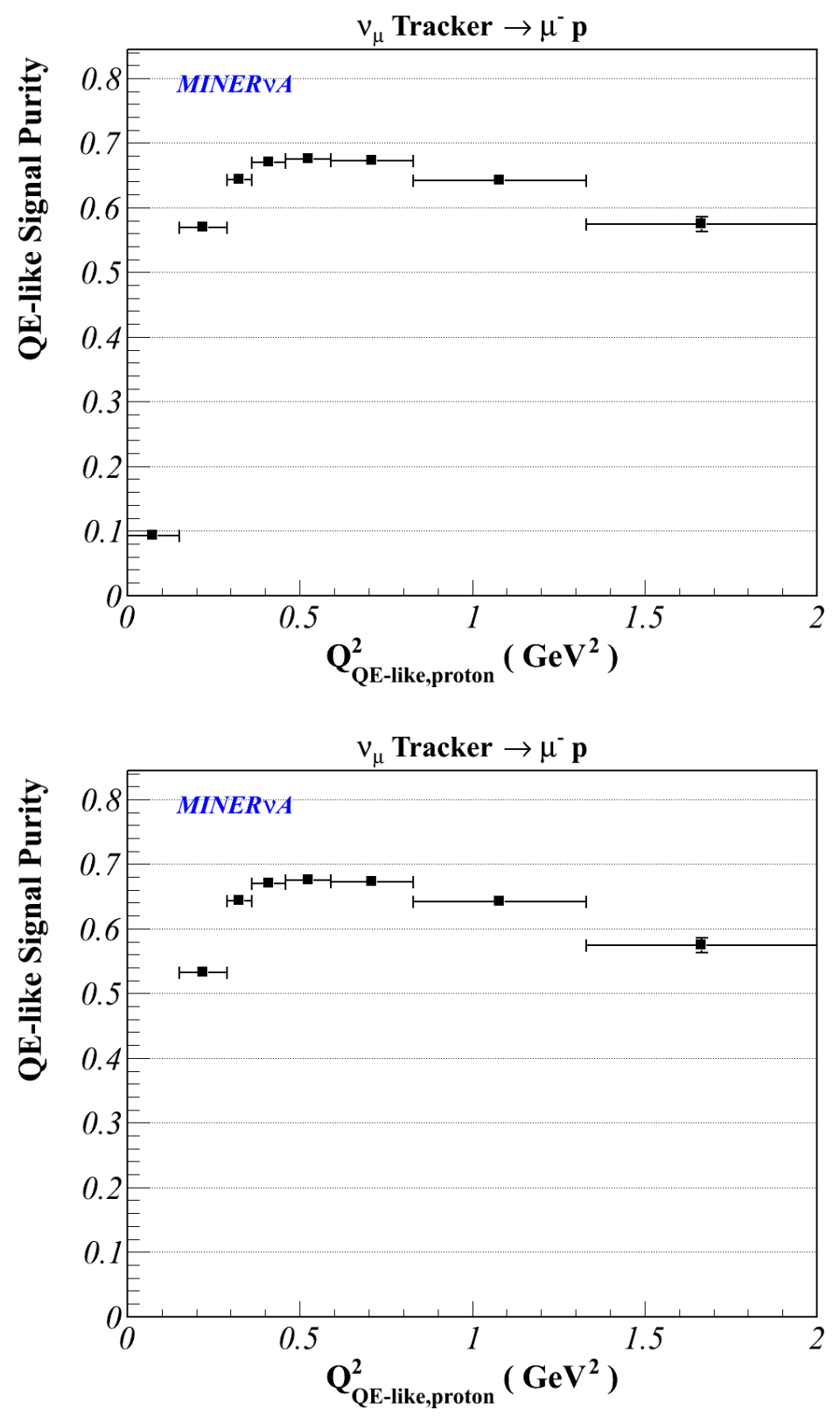

Figure C.2. Plotted is the purity as function of $\mathrm{Q}_{\mathrm{QE} \text {,proton }}^{2}$ for the $\nu_{\mu}$ QE-like signal without (top) and with (bottom) the condition on the proton's momentum (at least one proton in the final state with momentum greater than $450 \mathrm{MeV} / \mathrm{c}$ ). 

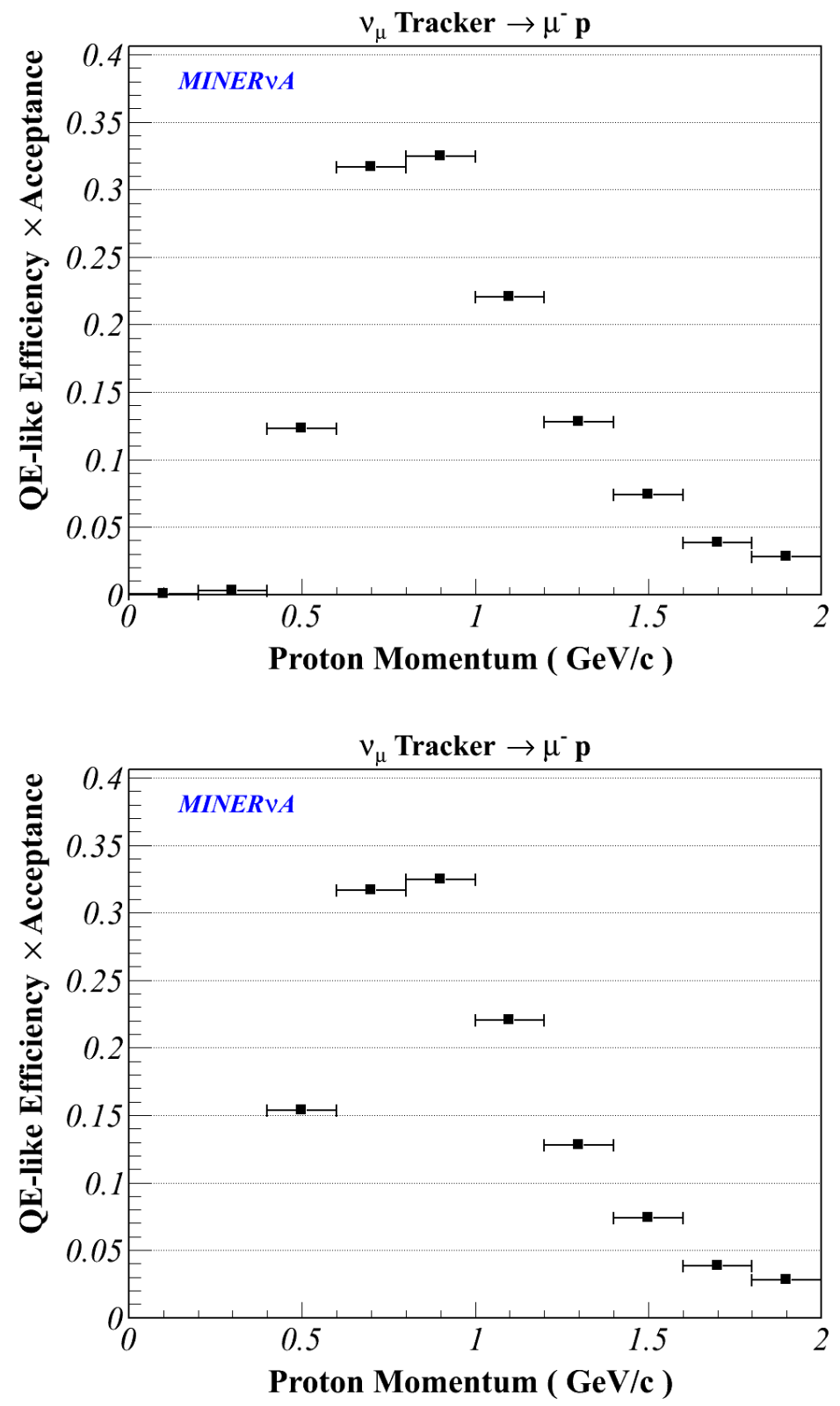

Figure C.3. Plotted is the efficiency as function of the proton's momentum for the $\nu_{\mu}$ QE-like signal without (top) and with (bottom) the condition on the proton's momentum (at least one proton in the final state with momentum greater than 450 $\mathrm{MeV} / \mathrm{c})$. 

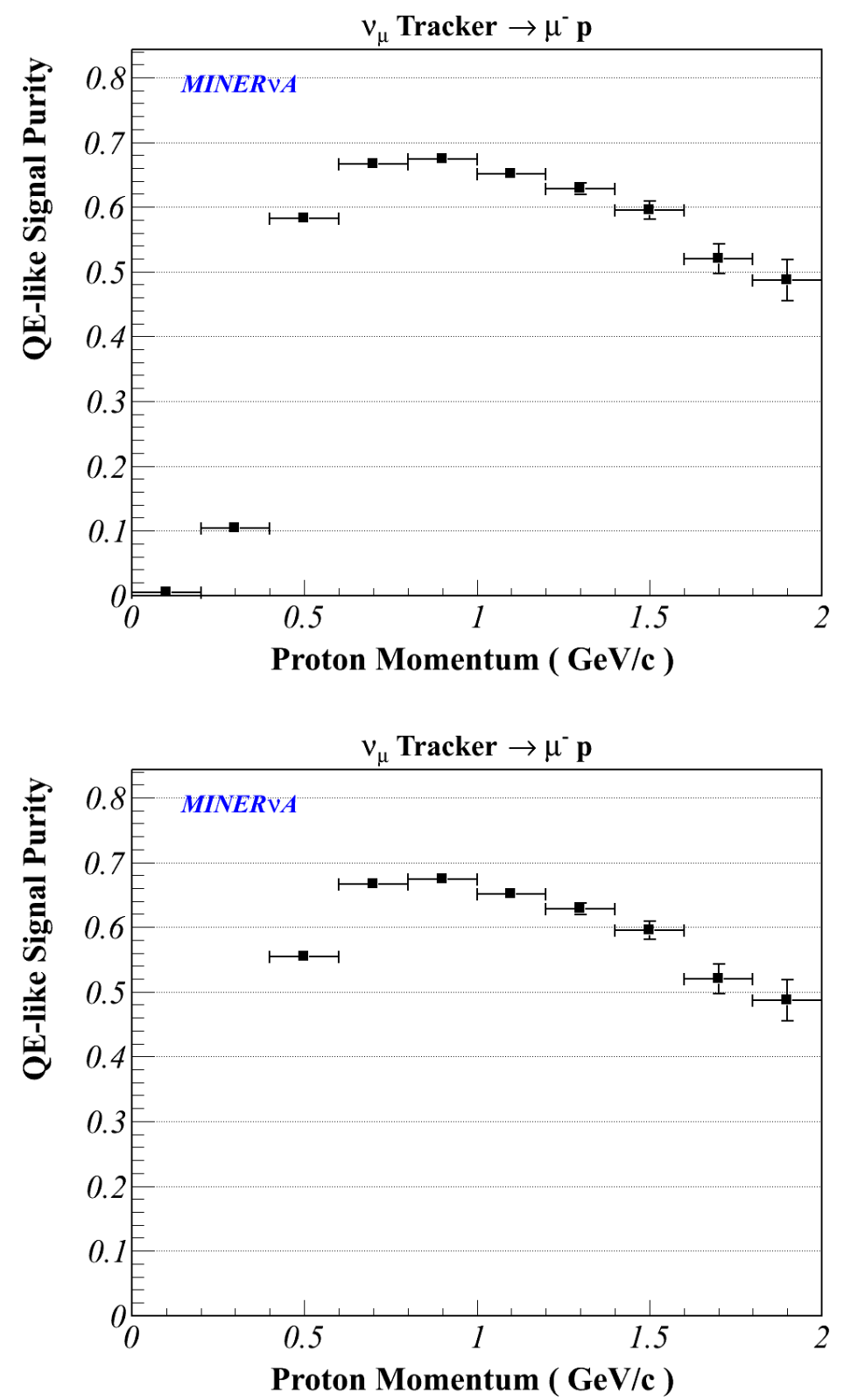

Figure C.4. Plotted is the purity as function of the proton's momentum for the $\nu_{\mu}$ QE-like signal without (top) and with (bottom) the condition on the proton's momentum (at least one proton in the final state with momentum greater than 450 $\mathrm{MeV} / \mathrm{c})$. 

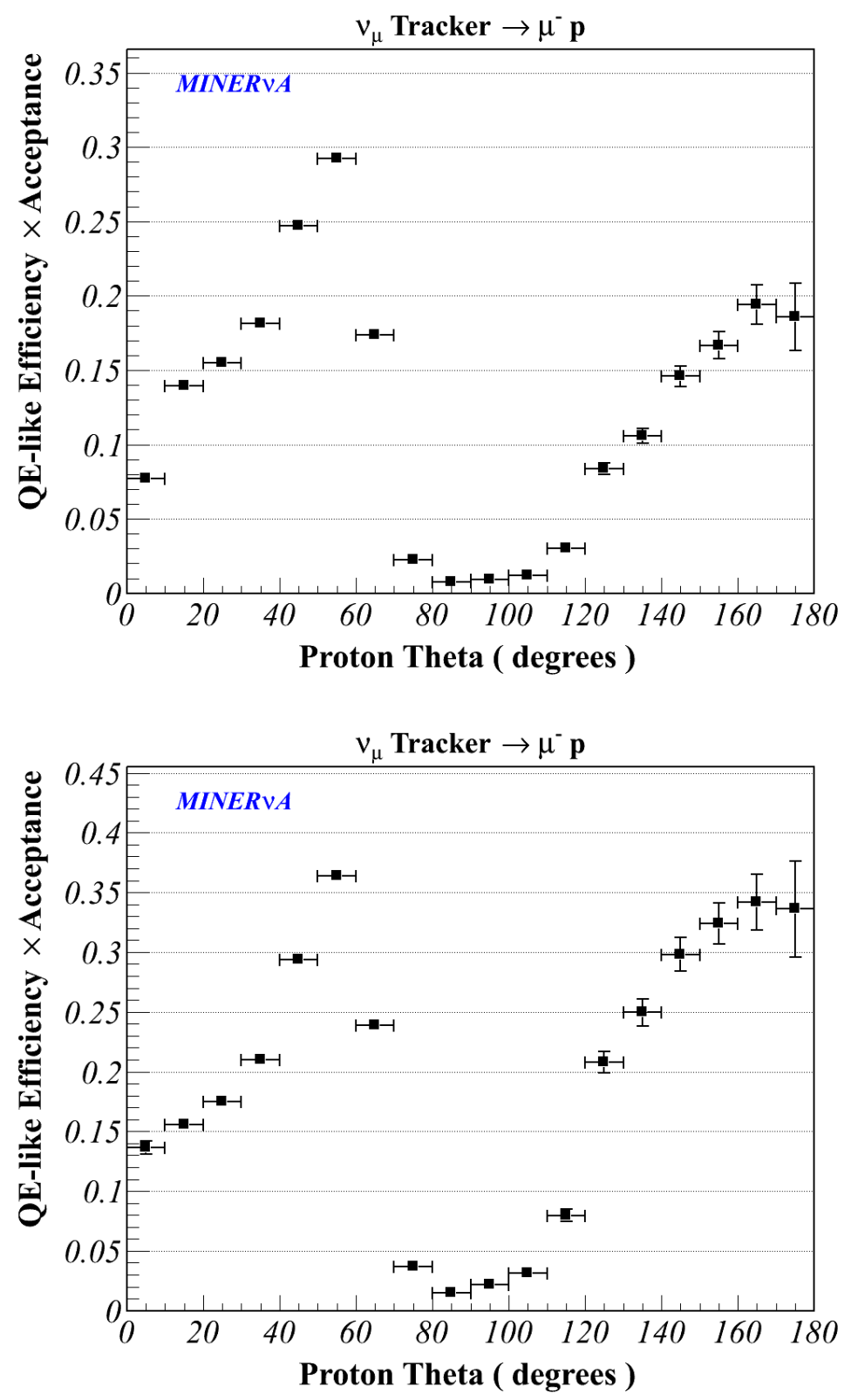

Figure C.5. Plotted is the efficiency as function of the proton's polar angle for the $\nu_{\mu}$ QE-like signal without (top) and with (bottom) the condition on the proton's momentum (at least one proton in the final state with momentum greater than 450 $\mathrm{MeV} / \mathrm{c})$. 

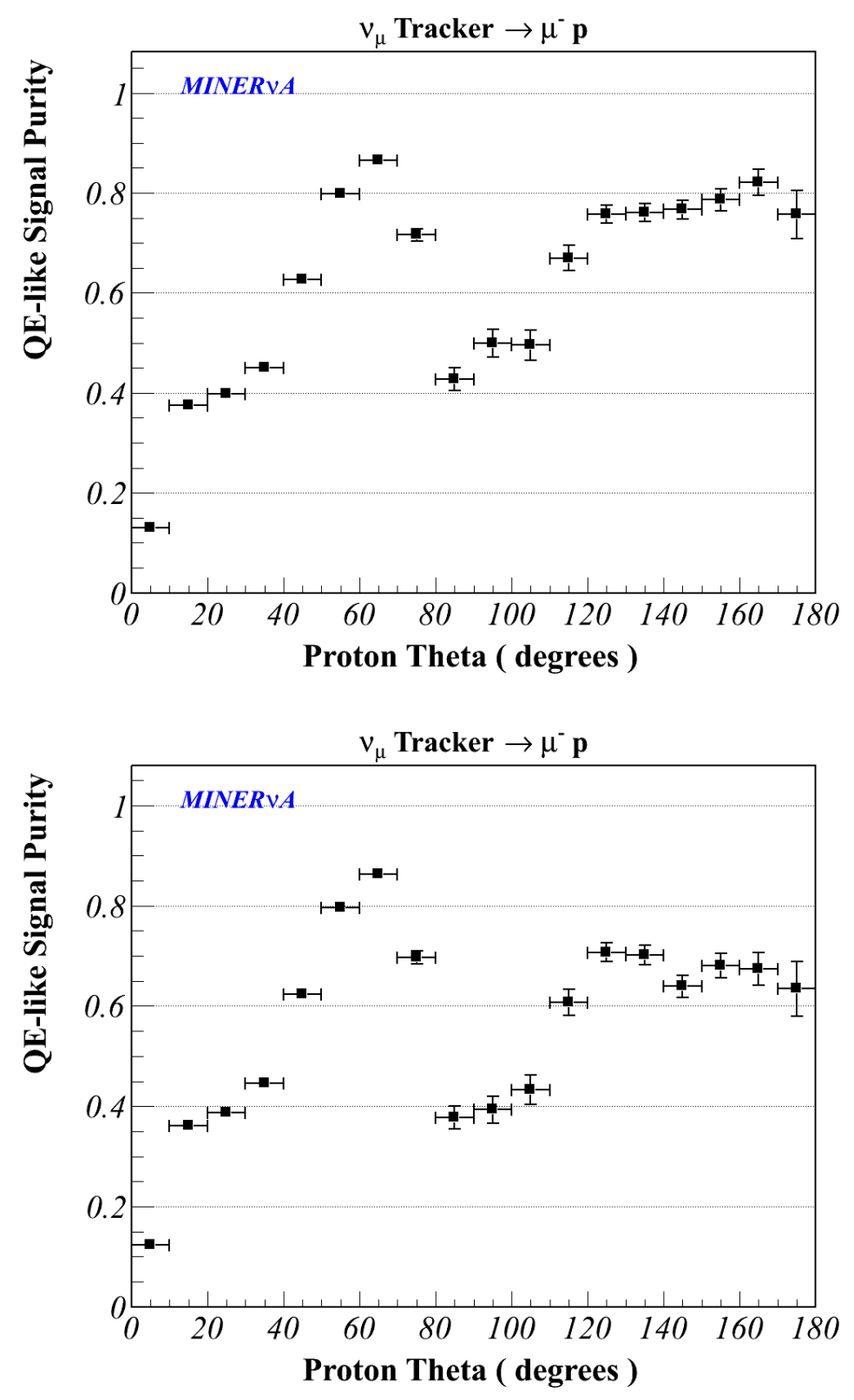

Figure C.6. Plotted is the purity as function of the proton's polar angle for the $\nu_{\mu}$ QE-like signal without (top) and with (bottom) the condition on the proton's momentum (at least one proton in the final state with momentum greater than 450 $\mathrm{MeV} / \mathrm{c})$. 


\title{
APPENDIX D
}

\section{THE MINER $\nu$ A COLLABORATION}

\author{
G. Tzanakos \\ University of Athens
}

J. Cravens, M. Jerkins, S. Kopp, J. Ratchford

University of Texas at Austin

D.A.M. Caicedo, C.M. Castromonte, H. da Motta, G.A. Fiorentini, K. Hurtado, J.L. Palomino

Centro Brasileiro de Pesquisas Físicas

\author{
D.W. Schmitz \\ University of Chicago \\ J. Grange, J. Mousseau, B. Osmanov, H. Ray \\ University of Florida
}

D. Boehnlein, R. DeMaat, N. Grossman, D.A. Harris, J.G. Morfín, J. Osta, E. A.

Paschos, L. Rakotondravohitra, P. Rubinov, F.D. Snider, R. Stefanski

\section{Fermilab}

A. Blondel, A. Bravar, Y. Karadzhov, A. Korzenev, C. Martin Mari University of Geneva

J. Felix, A. Higuera, Z. Urrutia, E. Valencia, G. Zavala 


\title{
Universidad de Guanajuato
}

\author{
M.E. Christy, M. Datta, C. Keppel, W. Tan, T. Walton, L. Y. Zhu \\ Hampton University
}

\begin{abstract}
A. Butkevich, S.A. Kulagin
Inst. Nucl. Reas. Moscow
\end{abstract}

G. Niculescu, I. Niculescu

James Madison University

\author{
E. Maher \\ Mass. Col. Lib. Arts
}

L. Fields, B. Gobbi, J.A. Hobbs, C.E. Patrick, L. Patrick, H. Schellman

Northwestern University

\author{
N. Tagg \\ Otterbein University
}

S. Boyd, S.A. Dytman, B. Eberly, Z. Isvan, C.L. McGivern, D. Naples, V. Paolone, L. Ren University of Pittsburgh

G.A. Díaz , A.M. Gago, J.P. Velásquez

Pontificia Universidad Catolica del Peru 


\author{
R. Napora \\ Purdue University Calumet
}

S. Avvakumov, A. Bodek, R. Bradford, H. Budd, J. Chvojka, M. Day, H. Lee, L. Loiacono, S. Manly, C.M. Marshall, K.S. McFarland, A.M. McGowan, A. Mislivec, J. Park, G.N. Perdue, P.A. Rodrigues, J. Wolcott

University of Rochester

\begin{abstract}
G. J. Kumbartzki, T. Le, R.D. Ransome, E.C. Schulte, B.G. Tice Rutgers University
\end{abstract}

O. Altinok, H. Gallagher, T. Kafka, W.A. Mann, W. P. Oliver Tufts University

\author{
C. Simon, B.P.Ziemer \\ University of California at Irvine
}

\author{
R. Gran, M. Lanari \\ University of Minnesota at Duluth \\ M. Alania, C.J. Solano Salinas \\ Universidad Nacional de Ingeniería
}

W.K. Brooks, G. Maggi, J. Miller, C. Peña, I.K. Potashnikova, F. Prokoshin Universidad Técnica Federico Santa María

L. Aliaga, J. Devan, M. Kordosky, J.K. Nelson, J. Walding, D. Zhang 
College of William and Mary 


\section{REFERENCES}

[1] G.A.Fiorentini, et. al. Measurement of Muon Neutrino Quasi-Elastic Scattering on Hydrocarbon Target at $\mathrm{E}_{\nu} \sim 3.5 \mathrm{GeV}$. Phys.Rev.Lett. 111:022502 (2013).

[2] L. Fields, et. al. Measurement of Muon AntiNeutrino Quasi-Elastic Scattering on Hydrocarbon Target at $\mathrm{E}_{\nu} \sim 3.5 \mathrm{GeV}$. Phys.Rev.Lett. 111:022501 (2013).

[3] N. Solomey. The Elusive Neutrino: A Subatomic Detective Story. W H Freeman and Company, (1997).

[4] F. Halzen and A.D. Martin. Quarks and Leptons: An Introductory Course in Modern Particle Physics. John Wiley and Sons, (1984).

[5] B. R. Martin. Nuclear and Particle Physics: An Introduction. John Wiley and Sons, (2006).

[6] M. Amarian. Introduction to Particle Physics, (2007). Internal Handout Document.

[7] F.P. An , et al. Improved Measurement of Electron Antineutrino Disappearance at Daya Bay. Chin Phys. C37 (2013).

[8] K. Abe, et al. Observation of Electron Neutrino Appearance in a Muon Neutrino Beam. Phys.Rev.Lett. 112:061802 (2014).

[9] Y. Koshio. Other neutrino oscillation measurements. arxiv:1301.3548[hepex] (2013).

[10] T. Schwetz M.A. Tortola and J.W.F. Valle. Three-flavour neutrino oscillation update. New J.Phys. 10:113011 (2008). 
[11] D.M. Webber. An Improved Measurement of Electron Antineutrino Disappearance at Daya Bay. Nucl.Phys.Proc.Suppl. 233:96-101 (2012).

[12] D.G. Michael et al. The Magnetized steel and scintillator calorimeters of the MINOS experiment. Nucl.Instrum.Meth. A596:190-228 (2008).

[13] S.D. Ayres et al. The NOvA Technical Design Report. (2007). FERMILABDESIGN-2007-01.

[14] K. Abe et al. Indication of Electron Neutrino Appearance from an Accelerator-Produced Off-Axis Muon Neutrino Beam. Phys.Rev.Lett. 107:041801 (2011).

[15] R.N. Cahn and G. Goldhaber. The Experimental Foundations of Particle Physics. Cambridge University Press, (2003).

[16] K.M. Graczyk. Relevance of Two Boson Exchange Effect in Quasi-Elastic Charged Current Neutrino-Nucleon Interaction. arxiv:1308.5581[hep-ph] (2013).

[17] A.J.R Puckett. Recoil Polarization Measurements of the Proton Electromagnetic Form Factor Ratio to High Momentum Transfer. PhD thesis, Massachusetts Institute of Technology, February (2010).

[18] E.J. Beise. The Axial form-factor of the nucleon. Eur.Phys.J. A24S2:43-46 $(2005)$.

[19] L. Garbini. Neutrino Cross-sections in the Wide Range of Energies Relevant for Low-background Underground Experiments. rafael.ujf.cas. cz/school12/presentations/garbini.pdf, (2012). 24th Indian-Summer School. 
[20] H. Gallagher D. Harris A. Kartavtsev and E. Paschos. Neutral and Charged Current Neutrino-Nucleus Coherent Measurements. minerva. fnal.gov/notes/minnote_300_coh.pdf, (2004). MINERvA Internal Note 300.

[21] C.H. LLewellyn Smith. Neutrino Reactions At Accelerator Energies. Phys.Rept. 3:261-379 (1972).

[22] E.D. Commins and P.H. Bucksbaum. Weak Interactions of Leptons and Quarks. Press Syndicate of the University of Cambridge, (1983).

[23] Y. Kurihara. Study of the Weak Nucleon Axial-Vector Form Factor and the Second-Class Current in Quasielastic Neutrino and Antineutrino Interactions. PhD thesis, January (1987). Workshop at Hiroshima University.

[24] S.V. Belikov et al. Quasielastic Neutrino and Anti-neutrinos Scattering: Total Cross-sections Axial Vector Form-factor. Z.Phys. A320:625 (1985).

[25] T.J. Leitner. Neutrino Interactions with Nucleons and Nuclei. PhD thesis, Institut fur Theoretische Physik, September (2005).

[26] H. Budd, A. Bodek, and J. Arrington. Modeling Quasi-elastic Form Factors for Electron and Neutrino Scattering. arxiv:0308005v2[hep-ex] (2003).

[27] R. Bradford , A. Bodek , H. Budd, and J. Arrington. A New Parametrization of the Nucleon Elastic Form Factors. Nucl.Phys.Proc.Suppl. 159:127$132(2006)$.

[28] T. Katori. A Measurement of the Muon Neutrino Charged Current QuasiElastic A Test of the Lorentz Violation with the MiniBooNE Experiment. PhD thesis, Indiana University, December (2008). 
[29] V. Bernard, L. Elouadrhiri, and U.G. MeiBner. Axial Structure of the Nucleon: Topical Review. J.Phys. G28:R1-R35 (2001).

[30] A. Bodek, S. Awakumov , R. Bradford, and H. Budd. Extraction of the Axial Nucleon Form Factor from Neutrino Experiments on Deuterium. J.Phys.Conf.Ser. 1110:082004 (2008).

[31] NOMAD: Neutrino Oscillation Magnetic Detector WA96. www . nu . to . infn . it/exp/all/nomad, (2013).

[32] Booster Neutrino Experiment. www-boone.fnal.gov/index.html, (2013).

[33] Y. Hayato. Brief Introduction of the Neutrino Event Generators. Kamioka observatory, ICRR, The University of Tokyo.

[34] A.V. Butkevich and S.P. Mikheyev. Test of Fermi gas model and plane-wave impulse approximation against electron-nucleus scattering data. Phys.Rev. C72:025501 (2005).

[35] C. Juszczak , J.T. Sobczyk , and J. Zmuda. On extraction of value of axial mass from MiniBooNE neutrino quasi-elastic double differential cross section data. Phys.Rev. C82:045502 (2010).

[36] MiniBooNE Collaboration. First Measurement of the Muon Neutrino Charged Current Quasielastic Double Differential Cross Section. Phys.Rev. D81:092005 (2010).

[37] M.B. Barbaro. Nuclear effects in charged-current quasielastic neutrinonucleus scattering. J.Phys.Conf.Ser. 336:012024 (2011).

[38] A.M. Ankowski. Breakdown of the impulse approximation and its consequences: The Low-Q**2 problem. PoS NUFACT08:118 (2008). 
[39] D. Higinbotham , E. Piasetzky , and M. Strikman. Short-Range NucleonNucleon Correlations. www. aps.org/units/dnp/research/correlations . cfm, (2013).

[40] L. Weinstein. The Nucleons Went Two By Two: Short Range Correlations in Nuclei, (2009). Lecture on Quarks and Nuclear Physics.

[41] J.T. Sobczyk. Theory Overview on Neutrino-nucleon (-nucleus) Scattering, (2013). NuFact13.

[42] J. Arrington , D. W. Higinbotham , G. Rosner , and M. Sargsian. Hard probes of short-range nucleon-nucleon correlations. Prog.Part.Nucl.Phys. 67 (2012).

[43] M.B. Barbaro et. al. Superscaling in electron-nucleus scattering and its link to CC and NC QE neutrino-nucleus scattering. arxiv:1303.6508[nucl-th] (2013)

[44] J.T. Sobczyk. Multinucleon ejection model for two-body current neutrino interaction. Phys.Rev. C86:015504 (2012).

[45] M. Hartz and K. McFarland. Energy reconstruction and near detectors, (2013). HyperK Meeting, Kavil IPMU.

[46] A. Bodek , H.S. Budd, and M.E. Christy. Resolving the Axial Mass Anomaly in neutrino Scattering. AIP Conf.Proc. 1441:420-422 (2012).

[47] M. Martini, M. Ericson, and G. Chanfray. Energy reconstruction effects in neutrino oscillation experiments and implications for the analysis. Phys.Rev. D87:013009 (2013).

[48] D. Schmitz. Quasi-Elastic Scattering of Neutrinos and Antineutrinos at MINERvA, (2013). Joint Experimental-Theoretical Physics Seminar. 
[49] S.A. Wood. Neutrino / Electron Scattering Comparison. In 2nd Internation Workshop on Neutrino-Nucleus Interactions in the Few GeV Region, 2002.

[50] T. Golan, C. Juszczak, and J.T. Sobczky. Final State Interactions Effects in Neutrino-Nucleus Interactions. Phys.Rev. C86:015505 (2012).

[51] L. Aliaga et al. Design, Calibration, and Performance of the MINERvA Detector. Nucl.Instrum.Meth. A743:130 (2014).

[52] R. M. Zwaska. Accelerator Systems and Instrumentation for the NuMI Neutrino Beam. PhD thesis, The University of Texas at Austin, December $(2005)$.

[53] B.G. Tice. Measurement of Nuclear Dependence in Inclusive Charged Current Neutrino Scattering. PhD thesis, Rutgers, The State University of New Jersey, (2014).

[54] Fermi National Accelerator Laboratory. The Fermilab Main Injector Technical Design Handbook, (1994). FERMILAB-DESIGN-1994-01.

[55] J. Chvojka. Anti-Neutrino Charged Current Quasi-Elastic Scattering in MINERvA. PhD thesis, University of Rochester, (2012).

[56] Bruce Baller. NuMI Rookie Book. www-bdnew.fnal.gov/operations/ rookie_books/NuMI_v1.pdf, (2005).

[57] T. Asher and S. Baginski. Main Injector Rookie Book. www-bdnew. fnal. gov/operations/rookie_books/Main_Injector_v1.1.pdf, (2003).

[58] S. Kopp. The NuMI Neutrino Beam at Fermilab. arxiv:0508001 (2005). FERMILAB-CONF-05-093-AD. 
[59] S. Childress. The NuMI Proton Beam at Fermilab Successes and Challenges. Iss.fnal.gov/archive/2008/conf/fermilab-conf-08-380-ad. pdf, (2008). FERMILAB-CONF-080380-AD.

[60] R. Zwaska et. al. Beam-based alignment of the numi target station components at fnal. Nucl.Instrum.Meth. A568:548-560 (2006).

[61] Fermi National Accelerator Laboratory. NuMI Technical Design Handbook. www-numi.fnal.gov/numwork/tdh/thd_index.html, (2004).

[62] J.K. Nelson. Private communications, (2014).

[63] S. Kopp et. al. Ion chambers for monitoring the numi beam at fnal. In Proceeding of EPAC 2004, Lucerne, Switzerland, (2004).

[64] G.N. Perdue et al. The minerva data acquisition system and infrastructure. Nucl.Instrum.Meth. A694:179-192 (2012).

[65] P. Adamson et. al. Neutrino and Antineutrino Inclusive Charged-Current Cross section Measurements with the MINOS Near Detector. Phys.Rev. D81:072002 (2010).

[66] C.M. Marshall. The MINERvA Strip-to-Strip Calibration, (2012). MINERvA Technical Note.

[67] Z. Pavlovic. G4numi beam Monte Carlo, (2010). LBNE Beam Simulations Meeting.

[68] L. Aliaga. MINERvA Flux: Current Uncertainties And Future Plans, (2012). Flux Measurement and Determination in the Intensity Frontier Era Neutrino Beams Worshop. 
[69] L.J. Loiacono. Measurement of the muon neutrino inclusive charged current cross section on iron using the MINOS detector. PhD thesis, University of Texas at Austin, (2010).

[70] A. Bodek et al. Methods to Determine Neutrino Flux at Low Energies: Investigations of the low $\nu$ Method. Eur.Phys.J C72:1973 (2012).

[71] J. Park. Neutrino-Electron Scattering in MINERvA for Constraining the NuMI Neutrino Flux, (2013). MINERvA Internal Note No. 041.

[72] M. Kordosky, et. al. A brief documentation of the flux used in the may 2013 analyses, (2013). MINERvA Internal Note.

[73] C. Alt , et. al. Inclusive production of charged pions in $\mathrm{p}+\mathrm{p}$ collisions at $158 \mathrm{GeV} / \mathrm{c}$ beam momentum. Eur.Phys.J. C45:343-381 (2006).

[74] Barton et. al. Experimental study of the a-dependence of inclusive hadron fragmentation. Phys.Rev. D27:2580 (1983).

[75] C. Andreopoulos, et. al. The genie neutrino monte carlo generator. Nucl.Instrum.Meth. A614:87-104 (2010).

[76] P.A. Rodrigues and B. G. Tice. Extracting Cross Sections from a GENIE Event Sample, (2013). MINERvA Technical Note.

[77] A. Bodek. Vector and Axial Form Factors Applied to Neutrino Quasi-Elastic Scattering. www.jlab.org/conferences/neutrino/talks/bodek_a.pdf, (2006). Workshop on Intersections of Nuclear Physics with Neutrinos and Electrons.

[78] D. Rein and L.M. Sehgal. Neutrino-excitation baryon resonanes and single pion production. Annals of Physics 133:79-153 (1981). 
[79] L. Alvarez-Ruso ,et. al. The GENIE Neutrino Monte Carlo Generator: Physics and User Manual, (2013).

[80] C. Andreopoulos. The GENIE Neutrino Monte Carlo Generator, (2009). 45th Karpacz Winter School in Theoretical Physics.

[81] S. Agostinelli, et. al. Geant4: A simulation toolkit. Nucl.Instrum.Meth. A506:250-303 (2003).

[82] R. Fruhwirth. Application of Kalman filtering to track and vertex fitting. Nucl.Instrum.Meth. A262:444-450 (1987).

[83] Ar. Belkov. Implementation of the hough transform for 3d track reconstruction in drift chambers. arxiv:0201071[physic.comp-ph] (2002).

[84] W. Waltenberger. Adaptive vertex reconstruction. Technical Report CMSNOTE-2008-033, CERN, Geneva, (2008).

[85] W. R. Leo. Techniques for Nuclear and Particle PHysics Experiments. Speinger-Verlag, (1994).

[86] D.E. Groom , et. al. Muon stopping power and range tables 10-100 tev. Atomic Data and Nuclear Data Tables. 76:2 (2001).

[87] S. Seltzer and M. Berger. Atomic and nuclear properties of materials. Atomic Data and Nuclear Data Tables. 30:261 (1984).

[88] N. Tagg , et. al. Arachne - a web-based event viewer for minerva. Nucl.Inst.Meth. 676:44-49 (2012).

[89] B. Eberly. Probing Nuclear Physics with Neutrino Pion Production at MINERvA, (2014). Joint Experimental-Theoretical Physics Seminar. 
[90] T. Adye. Unfolding algorithms and tests using roounfold. Proceedings of the PHYSTAT 2011 Workshop (2011).

[91] B. G. Tice. TargetUtils, (2013). MINERvA Technical Note No. 021.

[92] M. Kordosky. Error bands from the many universes method, (2012). MINERvA Internal Note.

[93] R. P. Feynman. Very high-energy collisions of hadrons. Phys.Rev.Lett. 23:1415 (1969).

[94] J. Wolcott. Uncertainties due to feynman scaling of na49 data to numi energy, (2013). MINERvA Internal Note 015.

[95] Ž. Pavlović. Observation of disapperance of muon neutrinos in the NuMI beam. PhD thesis, (2008).

[96] C. Andreopoulos, et. at. Update Cross Section Model Uncertainties for the Charged Current Analysis, (2007). MINERvA Internal Note.

[97] J.P.Velásquez, S.A. Dytman, and A.M. Gago. Geant4 hadronic uncertainties, 2012. MINERvA Internal Note docdb-8249v1.

[98] T. Walton. Minerva muon energy scale, (2012). MINERvA Internal Note docdb-7595.

[99] A. Mislivec. Meu systematics and approval plots, (2012). MINERvA Internal Note docdb-7571.

[100] R. Gran. Birk's law parameter from minerva testbeam data, (2013). MINERvA Internal Note 037.

[101] B. Eberly. Birks systematics, (2014). MINERvA Internal Note docdb$9526 \mathrm{v} 3$. 
[102] R. Ransome. Minerva physical description, 2012. MINERvA Internal Note docdb-6016v7.

[103] D. Zhang. Micheltool updates, (2014). MINERvA Internal Note docdb9493v2.

[104] B.G. Tice. Tracker Mass Fraction Problem ( 1\%) Shortstop Muon Tracks ( 5\%), (2013). MINERvA Internal Note docdb-8824.

[105] C. Juszczak. Running nuwro. Acta Phys. Polon. B40:2507-2512 (2009).

[106] J. Sobczyk. Private communications, (2014).

[107] A. Bodek and J.L. Ritchie. Fermi-motion effects in deep-inelastic lepton scattering from nuclear targets. Phys.Rev. D23:1070 (1981).

[108] P.Lava, et. al. Nuclear transparencies in relativistic A(e,e' p) models. Phys.Lett. B595 (2004).

[109] M.E. Christy. Private communications, (2014). 